840 Post-Appendectomy Colorectal Adenocarcinomas Associate with Low Rates of Lymph Node Metastasis and High Rates of Mismatch Repair Deficiency

Menglei Zhu, Wei Xin. University Hospitals Case Medical Center, Case Western Reserve University, Cleveland, $\mathrm{OH}$.

Background: The vermiform appendix in humans is generally considered as a useless remnant. However, studies suggest that it serves as a "safe house" for biofilm formation to preserve and protect commensal bacteria needed for the epithelial mucosa and regulate immune response in colon. Also recent study suggests that an increased rate of colorectal adenocarcinoma (CRC) in associate with appendectomy. In this study, we tried to determine whether any histological features are specially associated with post-appendectomy CRCs.

Design: We reviewed all right side colectomy CRC specimens in one tertiary center between years of 2011-2015. Histological features including differentiation, mucinous components, Crohn's like reaction, angiolymphatic invasion, lymph node involvements were documented. MMR statuses have also been examined by IHC using a four-antibody panel (MLH1, MSH2, MSH6, and PMS2).

Results: 125 cases with appendix and 58 cases without appendix were identified. Mean age was 69.5 years for the cases with appendix and 71.6 year for the cases without appendix. Male verse Female ratio was 1:1.1 for the cases with appendix and 1:2 for the cases without appendix. The histological features with statistically significance are shown in the following table.

\begin{tabular}{|l|l|l|l|}
\hline & Cases with appendix & Cases without appendix & \\
\hline Mucinous components & $23.8 \%(10 / 42)$ & $75.8 \%(25 / 33)$ & $\mathrm{P}<0.05$ \\
\hline Lymph nodes Involvement & $44.2 \%(53 / 120)$ & $38.9 \%(21 / 54)$ & $\mathrm{P}<0.05$ \\
\hline MMR status & $21.6 \%(16 / 74)$ & $40.9 \%(9 / 22)$ & $\mathrm{P}<0.05$ \\
\hline
\end{tabular}

Conclusions: Though the data is not conclusive, we do observe a higher ratio of previous appendectomy in right colectomy CRC specimens comparing with that of in the general population. The CRCs status post appendectomy show higher rates of mucinous components, and MMR deficiency, but a lower rate of lymph node involvement. The study suggests that appendectomy may play a role in CRC development.

\section{Genitourinary Pathology (including Renal tumors)}

\section{Racial Disparity in Papillary Renal Cell Carcinoma}

Adebowale Adeniran, Abiodun Mafolasire, Aaron Hartman, Cayce Nawaf, Peter A Humphrey, Brian Shuch. Yale University School of Medicine, New Haven, CT. Background: Significant racial disparities exist in the incidence, distribution, and survival in renal cell carcinoma (RCC). Several studies have confirmed a higher distribution of papillary RCC (pRCC) in African Americans. The objective of this study was to evaluate if there were differences in pRCC subtypes between Caucasians and African Americans

Design: Using an IRB approved single institutional database, we retrieved all cases with previously designated pRCC. All individual cases were reviewed for designation of papillary subtype as well as clinico-pathologic features. Only those subjects that underwent nephrectomy were included for analyses. Clinical data was collected from electronic medical records. Comparisons were made between Caucasians and African Americans using chi squared and independent T-testing.

Results: A total of 122 subjects met the inclusion criteria (101 male and 21 female). The clinico-pathologic characteristics of all tumors are summarized in Table 1. African Americans were younger $(\mathrm{p}=0.009)$ at presentation and had larger tumors $(\mathrm{p}=0.052)$. There was no significant difference in the pRCC subtype distribution. Nodal metastasis occurred more frequently in African Americans $(\mathrm{p}=0.034)$ and there was a trend to advanced T stage $(\mathrm{p}=0.072)$ and distant metastasis $(\mathrm{p}=0.083)$. There was no significant difference in tumor grade, presence of necrosis or microvascular invasion.

\begin{tabular}{|c|c|c|c|c|}
\hline & & $\begin{array}{l}\text { Caucasian } \\
\mathrm{n}=80\end{array}$ & $\begin{array}{l}\text { African } \\
\text { American } n=42\end{array}$ & $p$ value \\
\hline Age & Mean \pm SD & $60 \pm 14.8$ & $52.6 \pm 12.8$ & $\mathrm{p}=0.009$ \\
\hline Sex & $\begin{array}{l}\text { Male } \\
\text { Female }\end{array}$ & \begin{tabular}{|l|}
65 \\
15
\end{tabular} & $\begin{array}{l}36 \\
6\end{array}$ & $\mathrm{p}=.535$ \\
\hline Tumor Size & Mean \pm SD & $3.8 \pm 2.6$ & $4.8 \pm 4.1$ & $\mathrm{p}=0.052$ \\
\hline Histology & $\begin{array}{l}\text { Type 1 } \\
\text { Type 2 } \\
\text { NOS }\end{array}$ & $\begin{array}{l}42(54.5 \%) \\
27(35.1 \%) \\
8(10.4 \%)\end{array}$ & $\begin{array}{l}22(53.7 \%) \\
12(29.3 \%) \\
7(17.1 \%)\end{array}$ & $\mathrm{p}=0.544$ \\
\hline ISUP Grade & $\begin{array}{l}1 \\
2 \\
3 \\
4\end{array}$ & \begin{tabular}{|l|}
3 \\
49 \\
27 \\
0 \\
\end{tabular} & $\begin{array}{l}3 \\
25 \\
13 \\
1\end{array}$ & $\mathrm{p}=0.455$ \\
\hline T Stage & $\begin{array}{l}\mathrm{T} 1 \\
\mathrm{~T} 2 \\
\mathrm{~T} 3 \\
\mathrm{~T} 4 \\
\end{array}$ & \begin{tabular}{|l|}
64 \\
12 \\
3 \\
0 \\
\end{tabular} & $\begin{array}{l}32 \\
4 \\
3 \\
3 \\
\end{array}$ & $\mathrm{p}=0.072$ \\
\hline $\mathrm{N}$ & $\begin{array}{l}\text { N0/X } \\
\text { N1 }\end{array}$ & \begin{tabular}{|l|}
78 \\
2
\end{tabular} & $\begin{array}{l}37 \\
5\end{array}$ & $\mathrm{p}=0.034$ \\
\hline M & $\begin{array}{l}\text { M0 } \\
\text { M1 }\end{array}$ & \begin{tabular}{|l|}
77 \\
2 \\
\end{tabular} & $\begin{array}{l}37 \\
5 \\
\end{array}$ & $\mathrm{p}=0.083$ \\
\hline Necrosis & $\begin{array}{l}\text { No } \\
\text { Yes }\end{array}$ & \begin{tabular}{|l|}
43 \\
32 \\
\end{tabular} & $\begin{array}{l}22 \\
16 \\
\end{array}$ & $\mathrm{p}=0.955$ \\
\hline $\begin{array}{l}\text { Microvascular } \\
\text { invasion }\end{array}$ & $\begin{array}{l}\text { No } \\
\text { Yes }\end{array}$ & \begin{tabular}{|l|}
75 \\
1
\end{tabular} & $\begin{array}{l}41 \\
1\end{array}$ & $\mathrm{p}=0.668$ \\
\hline
\end{tabular}

Conclusions: In a single institutional series, we did not find racial differences in the subtype distribution of pRCC. However we found that African Americans with pRCC were significantly younger and had more nodal involvement. There was also a trend towards larger tumor size, higher stage and distant metastasis. Due to the low incidence of pRCC and a relatively low number of African American patients in this series, larger cohorts or multi-institutional efforts are needed to further analyze differences in pathologic characteristics in $\mathrm{pRCC}$ by race.

842 Neuroendocrine Carcinoma: A Genomically Distinctive Form of Prostate Cancer

Sanaz Ainechi, Bryan Firestone, Julia A Elvin, Siraj M Ali, Jo-Anne Vergilio, James Suh, Badar Mian, Hugh Fisher, Tipu Nazeer, Vincent A Miller, Philip J Stephens, Jeffrey S Ross. Foundation Medicine, Inc., Cambridge, MA; Albany Medical College, Albany, NY. Background: Neuroendocrine carcinoma of the prostate (NCAP) is a rare, but highly aggressive form of poorly differentiated carcinoma of prostate (CAP). We compared the genomic landscape of NCAP with classic non-neuroendocrine CAP using comprehensive genomic profiling (CGP) focused on the search for genomic alterations (GA) that could influence therapy selection for patients with advanced disease.

Design: DNA was extracted from formalin fixed paraffin embedded tissue sections $(40 \mathrm{u}$ total) from 37 consecutive cases of immunohistochemistry (IHC)-confirmed relapsed/ metastatic NCAP and a control group of 509 cases of CAP. CGP was performed on hybridization-captured, adaptor ligation based libraries to a mean coverage depth of $583 \mathrm{X}$ for up to 315 cancer-related genes plus 37 introns from 14 genes frequently rearranged in cancer. GA included base substitutions (SUB), INDELs, copy number alterations (CNA) and fusions/rearrangements. Clinically relevant GA (CRGA) were defined as GA linked to drugs on the market or under evaluation in mechanism driven clinical trials.

Results: All $37(100 \%)$ cases of NCAP were positive for neuroendocrine markers on immunohistochemical staining. The median age of the males in this study was 65.1 years (range 43 to 83 years). All (100\%) of NCAP Cases were Stage IV at the time of CGP. Samples used for sequencing were obtained from the primary tumor in $9(24 \%)$ NCAP and from metastatic sites in $28(76 \%)(12$ liver, $6 \mathrm{LN}, 2$ each from bladder, pelvis and soft tissue, and 1 each from rectum, bone, urethra and ureter). There were 213 total GA ( $5.8 \mathrm{GA} / \mathrm{sample})$ and $47 \mathrm{CRGA}(1.3 \mathrm{CRGA} / \mathrm{sample})$. In comparison with CAP, NCAP featured an identical frequency of TMPRSS2:ERG fusions ( $32 \%$ vs $32 \%)$, a significantly higher frequency of GA in $T P 53(68 \% \mathrm{vs} 42 \%)(\mathrm{p}=0.011)$ and $R B 1(51 \%$ vs $7 \%)(\mathrm{p}<0.0001)$ and lower frequency of GA in $A R(8 \%$ vs $22 \%)(\mathrm{p}<0.05)$ and loss of PTEN (32\% vs $43 \%$ ) (NS). The most frequent CRGA in NCAP involved PTEN (32\%), BRCA2 (14\%), FGFR1 (5\%), PIK3CA (5\%) and AKT2 (3\%). No alterations in $B R A F$ were identified. Clinical responses to MTOR inhibitors in patients with MTOR pathway GA will be presented.

Conclusions: NCAP and CAP have similar TMPRSS2:ERG fusion frequencies, but differ in alteration frequencies for TP53, RB1, AR and PTEN. Multiple alterations in the MTOR pathway identified in this rare, but highly aggressive form of prostate cancer suggest that these patients may be candidates for MTOR inhibitor-based and other targeted therapies associated with BRCA2 GA (PARP inhibitors) and FGFR1 GA (FGFR inhibitors)

\section{Prognostic Significance of Depth of Invasion in Transurethral} Resection of Bladder Specimens

Mahmut Akgul, Matthew Bream, Nafiseh Janaki, Hammad Tashkandi, Gregory MacLennan. University Hospitals Case Medical Center, Cleveland, OH.

Background: Depth of invasion in transurethral resection of bladder (TURB) biopsies of Stage T1 urothelial cancer has been evaluated using measured depth of lamina propria (LP) invasion or by muscularis mucosa invasion. These are affected by biopsy orientation, variation in LP, and inconclusive presence of muscularis mucosa. Identifying tumor adjacent to detrusor muscle (TADM) is not affected by orientation or size of the LP and may be a better histologic indicator for advanced T1 disease.

Design: A language search of pathology archives from 1995-2015 using "transurethral resection" and "bladder" identified 2786 TURB cases. Pathology reports and clinical information was reviewed, and only those with $\mathrm{T} 1$ cancer at initial resection, urothelial histology, detrusor muscle (DM) present in biopsy, and adequate clinical records were included. H\&E and other available slides were reviewed, and patients were classified into two groups: LP invasion with TADM (group 1), or any other LP invasion without TADM (group 2). Further clinical information was obtained including, age, gender, treatment and disease course including all subsequent resections or cystectomy, and status of nodal or metastatic disease. Recurrence (subsequent resections with T1 or higher), rate of cystectomy, and progression (presence of T2 or higher or nodal/metastatic disease) were compared using Fisher's exact and chi-square test.

Results: 105 patients (M:F = 3.4:1, median age 74) met inclusion criteria. 20 patients had TADM (group 1), and 85 patients had no TADM (group 2). Disease recurrence was seen in 15 of 20 cases (75\%) in group 1 and 32 of 85 cases (37\%) in group 2, with significant difference $(\mathrm{p}=0.005)$. Disease progression was seen in 14 of $20(70 \%)$ in group 1 and 19 of 85 in group $2(22 \%)$, also statistically significant $(\mathrm{p}=0.04)$. Rates of lymph node positive disease at cystectomy or metastatic disease were significantly higher in group $1(8$ of $20,40 \%)$ than in group $2(9$ of $85,10 \%), p=0.003$. The cystectomy rate was higher in group 1 than group 2, although the difference was not significant $[8 / 20$ in group $1(40 \%)$ vs $22 / 85$ in group $2(25 \%), p=0.09]$.

Conclusions: This retrospective analysis of T1 disease in TURB specimens showed that LP invasion with TADM in TURB specimens is associated with higher rates of disease recurrence and progression. This means of subclassifying T1 bladder cancers is not affected by tissue orientation, variations in LP depth or presence of a muscularis mucosa layer, and may be a better prognostic tool. 
$\mathbf{8 4 4}$ Is Mast Cell Infiltration Specific to Interstitial Cystitis?

Yoshiyuki Akiyama, Daichi Maeda. Graduate School of Medicine, the University of Tokyo, Tokyo, Japan; Graduate School of Medicine, Akita University, Akita, Japan. Background: Mast cell infiltration has been documented as a key pathological features of interstitial cystitis (IC). However, previous evidence is based on eyemeasured mast cell counts, which can often be inaccurate and arbitrary. Furthermore, distinction between the two types of IC (Hunner-type IC (HIC), characterized by dense lymphoplasmacytic inflammation, and non-Hunner-type IC (NHIC), which shows little inflammatory changes) is not always made. Herein, we used image analysis software to precisely count the number of infiltrating mast cells in HIC and NHIC biopsy specimens. In addition, by comparing IC specimens and non-IC specimens, we attempted to elucidate whether mast cell infiltration is an IC-specific histological feature. Design: We quantified mast cells, T-lymphocytes, B-lymphocytes and plasma cells by immunohistochemistry in urinary bladder biopsy specimens taken from HIC patients $(\mathrm{N}=27)$, NHIC patients $(\mathrm{N}=39)$, and non-IC cystitis patients $(\mathrm{N}=23)$ using image analysis software. For HIC cases, biopsies from Hunner lesions and normal-looking background mucosa were evaluated. Mast cells, T-cells, B-cells, and plasma cells were identified by immunostaining with mast cell tryptase, CD3, CD20, and CD138 antibodies, respectively.

Results: A similar degree of lymphoplasmacytic infiltration was observed in HIC specimens and non-IC cystitis specimens, whereas most NHIC specimens contained very few lymphoplasmacytic cells. We observed no significant differences between the number of mast cells in HIC specimens and non-IC specimens. In NHIC specimens, mast cell count was significantly lower than HIC and non-IC cystitis specimens.
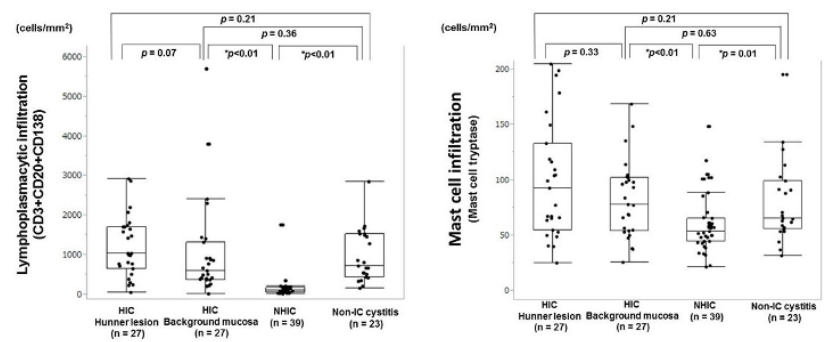

Conclusions: The results suggest that mast cell infiltration is not a specific histological feature of IC. HIC and NHIC are distinct in their pathophysiology, the former being a true inflammatory disorder, and the latter non-inflammatory. Since no increase in mast cells was observed in HIC samples compared to controls, the diagnostic significance of mast cell counts in IC biopsy specimens is low. The lower number of infiltrating mast cells in NHIC specimens most likely results from the disorder's non-inflammatory nature.

845 Application of ISUP Grading in a Series of Papillary Renal Cell Carcinoma Cases from a Tertiary Institution

Ashwin Akki, Rodrigo A Ledezma, Gladell P Paner. University of Chicago, Chicago, IL; Universidad de Chile, Santiago, Chile.

Background: In 2012, the ISUP consensus conference proposed a grading system based on nucleolar prominence for Papillary renal cell carcinoma (PRCC). We investigated this grading system in a large series of PRCC.

Design: 118 PRCC nephrectomies from 2002 to 2014 were graded according to the ISUP system. The primary endpoint was relapse-free survival (RFS). The estimated RFS was obtained using the Kaplan-Meier method and differences between estimates were assessed with the log-rank test.

Results: Mean patient age was 61 years (range $37-87$ years) for all PRCC. The PRCCs were type $1(62 / 118,53 \%)$, type $2(46 / 118,39 \%)$ and mixed 1 and $2(10 / 118,8 \%)$. The breakdown of ISUP grades is in table 1.

\begin{tabular}{|l|l|l|l|l|}
\hline ISUP Grade & 1 & 2 & 3 & 4 \\
\hline All PRCC & $9 / 118(7.6 \%)$ & $59 / 118(50 \%)$ & $42 / 118(35.6 \%)$ & $8 / 118(6.8 \%)$ \\
\hline Type 1 & $6 / 118(5 \%)$ & $36 / 118(30.5 \%)$ & $14 / 118(12 \%)$ & $6 / 118(5 \%)$ \\
\hline Type 2 & $3 / 118(2.6 \%)$ & $21 / 118(18 \%)$ & $21 / 118(18 \%)$ & $1 / 118(0.8 \%)$ \\
\hline Mixed type & $0 / 118(0 \%)$ & $2 / 118(1.5 \%)$ & $7 / 118(6 \%)$ & $1 / 118(0.8 \%)$ \\
\hline
\end{tabular}

RFS analysis showed that patients with PRCC type 2 did significantly worse than those with PRCC type 1 (Fig.1; $\mathrm{p}=0.03$ ) and that there was a tendency for patients with higher ISUP grades ( 3 and 4 ) to do worse (Fig. $2 ; \mathrm{p}=0.3$ ).
Figure 1. Relapse-free survival [PRCC type 1 vs. PRCC type 2] Log Rank: 0.03

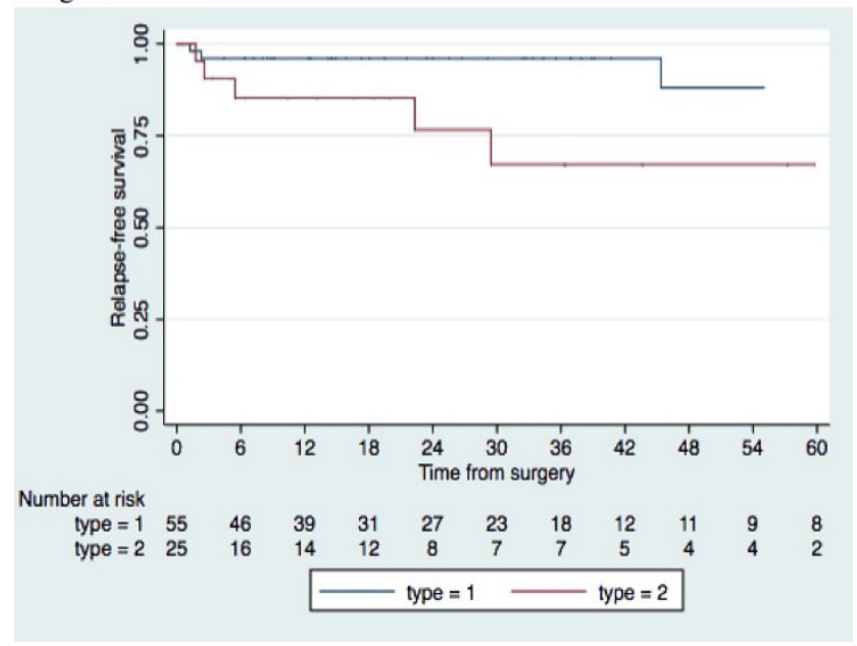

Figure 2. Relapse-free survival based on ISUP grades 1 to 4 Log Rank: 0.3

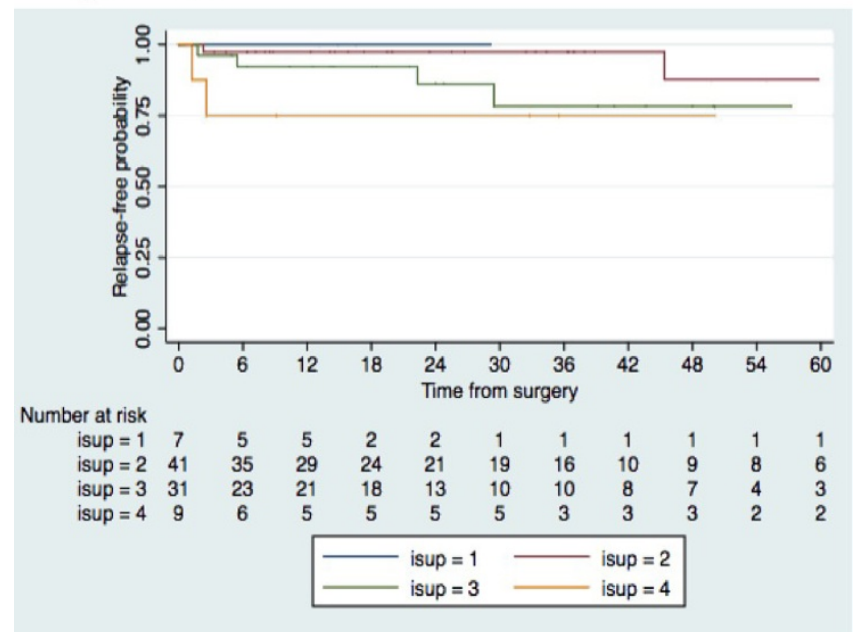

Conclusions: The ISUP grading system can be useful in predicting RFS in PRCC, particularly type 2 . However, this system needs to be validated in a larger series of cases.

846 Comprehensive Molecular Characterization of 412 Muscle Invasive Urothelial Carcinomas of the Bladder - Final Analysis of The Cancer Genome Atlas (TCGA) Project

Hikmat A Al-Ahmadie, Seth Lerner, Jaegil Kim, Gad Getz, David J Kwiatkowski, John Weinstein, Andrew Cherniack, Guangwu Guo, Rehan Akbani, Katherine A Hoadley, William Y Kim, Gordon Robertson, Andy Mungall, Toshinori Hinoue, Peter Laird, Jonathan E Rosenberg, Joaquim Bellmunt, Margaret B Morgan, Chad Creighton, Dmitry A Gordenin, Joshua M Stuart, Xiaoping Su, Raju Kucherlapati, Gordon Mills, Donna Hansel, Brian D Robinson, Bogdan A Czerniak, Victor E Reuter. The Cancer Genoma Atlas (TCGA) Bladder Cancer Working Group, New York, NY.

Background: Following the initial report of integrated molecular analysis of 131 urothelial carcinoma (UC) by The Cancer Genome Atlas (TCGA) Bladder Cancer Working Group (Nature 507:315, 2014), we now report on a cohort of 412 tumors from the TCGA project of chemotherapy-naïve, muscle-invasive UC of the bladder. This represents the largest sequencing project in $\mathrm{UC}$ to date.

Design: The cohort consisted of $412 \mathrm{UCs}$, all were fresh frozen and peripheral blood was used for germline DNA. Following strict clinical and pathologic quality control, tumors were analyzed for DNA copy number variants, somatic mutations by whole exome sequencing (WES), DNA methylation, mRNA, microRNA and (phospho-) protein expression, transcript splicing, gene fusions, viral integration, pathway perturbation, clinical correlates, and histopathologic evaluation.

Results: There was a high overall somatic mutation rate $(8.0 / \mathrm{Mb})$, median of 245 and mean of 348 coding region mutations per sample, which is the third highest among all TCGA cancers analyzed. There were 54 significantly mutated genes (SMGs), an increase from 32 in the original report. TP53 mutations were most common (49\%) along with mutations in a number of chromatin-modifying genes such as MLL2 (29\%), KDM6A (26\%), ARIDIA (25\%), MLL3 (19\%), EP300 (15\%), CREBBP (12\%) and MLL (11\%). Other cancer related genes with frequent mutations include PIK3CA (22\%), RB1 (17\%), FGFR3 (14\%), STAG2 (14\%), ATM (14\%), ELF3 (12\%), FAT1 (12\%), SPTAN1 (12\%), ERBB2 (12\%), ERBB3 (11\%), ASXL2 (10\%), ERCC2 (9\%), CDKN1A (9\%), TSC1 (8\%), CDKN2A (7\%), RHOB (6\%), NFE2L2 (6\%), RBM10 (5\%),HRAS (5\%), KRAS (4\%) 
and PTEN (3\%), some of which were not detected in or showed significantly increased frequency from the original report. APOBEC mutagenesis explained $70 \%$ of the mutation burden. Gene silencing by promoter DNA hypermethylation was identified in 167 genes with at least $5 \%$ frequency in the cohort. Previously identified 4 mRNA subtypes were predicted on the larger set with a similar proportion of samples in each subtype. Conclusions: This integrated molecular analysis of 412 TCGA samples validates and extends observations from the first cohort of 131 patients. It significantly increased our power to detect additional aberrations at lower frequency that were not identified in the original cohort. The results provide a robust basis for further functional studies to enhance our understanding of the biology of bladder cancer, and aid in the development of more precisely targeted therapies.

\section{Comprehensive Molecular Analysis of Plasmacytoid Variant} Urothelial Carcinoma

Hikmat A Al-Ahmadie, Gopa Iyer, Byron H Lee, Sasinya N Scott, Rohit Mehra, Aditya Bagrodia, Emmet J Jordan, Sizhi P Gao, Ricardo Ramirez, Eugene K Cha, Neil Desai, Emily C Zabor, Irina Ostrovnaya, Anuradha Gopalan, Ying-Bei Chen, Samson W Fine, Satish K Tickoo, Agnes Viale, Maria E Arcila, Guido Dalbagni, Jonathan E Rosenberg, Bernard H Bochner, Dean F Bajorin, Michael F Berger, Victor E Reuter, Barry S Taylor, David B Solit. Memorial Sloan Kettering Cancer Center, New York, NY.

Background: Plasmacytoid carcinoma (PC) of bladder is an aggressive histologic variant with high risk of recurrence and mortality. We previously reported frequent $\mathrm{CDH} 1$ mutations in $\mathrm{PC}$ but the molecular characteristics of this histologic variant are largely unknown. We performed comprehensive molecular analysis of a PC cohort and functional validatation of the role of $\mathrm{CDHI}$ loss in bladder cancer cell lines.

Design: We performed whole exome sequencing (WES), targeted deep sequencing (by MSK-IMPACT), sodium bisulfite sequencing of $\mathrm{CDH1}$ promoter and $\mathrm{IHC}$ for E-cadherin. We also performed $\mathrm{CDH} 1$ knockout by Clustered Regularly Interspersed Short Palindromic Repeats (CRISPR) in 2 cells lines to explore the role of E-cadherin loss on cell functions. We compared genetic profile of PC with that of the classic urothelial carcinoma (UC) from TCGA and our institutional prospectively sequenced cohort $(\mathrm{n}=183)$.

Results: In 31 PC tumors, $C D H 1$ somatic mutations were identified in 26 (13 nonsense, 6 frameshift, 4 splice site and 3 missense). In 4 of 5 remaining $C D H 1$ wild type tumors, $\mathrm{CDH} 1$ promoter hypermethylation was identified by bisulfite sequencing. Of the 6 PC samples undergoing WES, 2 harbored $C D H 1$ somatic mutations accompanied by copy-neutral loss of heterozygosity of wild-type allele. Other than $C D H 1$ alteration, genomic profile of PC was not substantially different from that of the combined $183 \mathrm{UC}$ tumors regarding frequency and co-mutation pattern. Notably, CDKN2A and KDM6A alterations were significantly less frequent in PC compared to UC

E-cadherin loss was found in invasive PC, whereas E-cadherin expression was retained within in situ component, suggesting that loss-of-function mutations and epigenetic silencing of $\mathrm{CDH} 1$ arise later in the molecular chronology of $\mathrm{PC}$ pathogenesis.

No germline $C D H 1$ mutations were identified in this cohort. By CRISPR/Cas9-mediated knockout of $C D H 1$ in $2 \mathrm{CDH} 1$ wild-type UC cell lines (RT4 and MGHU4), loss of E-cadherin expression resulted in increased cell migration and enhanced invasion across a Boyden chamber membrane.

Conclusions: $C D H 1$ alterations are pathognomonic of plasmacytoid carcinoma. Loss of E-cadherin expression, typically as a result of somatic alteration of the $\mathrm{CDH} 1$ gene, is associated with enhanced cellular migration and invasion, likely explaining the unique recurrence and poor clinical outcome in this disease. The co-mutation pattern is similar to classical UC, suggesting a common origin for both subtypes, with $\mathrm{CDH} 1$ alterations representing a distinct evolutionary path in plasmacytoid variant. In this cohort, we did not find associated germline $C D H 1$ alteration with PC.

\section{Meta-Analysis of Mesenchymal Characteristic-Related Gene} Expression in Bladder Cancer

Waleed Ali, Andre Kajdacsy-Balla. University of Illinois at Chicago, Chicago, IL. Background: Bladder cancer is among the most prevalent cancers, with around 70,000 new cases every year within the US alone. Of newly diagnosed cases, approximately a quarter of cases show signs of invasion into and past the detrusor muscle, with more cases infiltrating the detrusor muscle and beyond with time. Loss of E-cadherin and increased expression of N-cadherin, fibronectin, Twist, Snail, \& Slug are commonly associated with epithelial-mesenchymal transition (EMT), a key step towards invasion and metastasis.

Design: From the microarray database Oncomine ${ }^{\mathrm{TM}}$, mRNA expression levels regarding E-cadherin, N-cadherin, Slug, Snail, fibronectin, and Twist from datasets which had expression levels for both non-myoinvasive or myoinvasive bladder urothelial carcinoma were collected. The $\log 2$-median centered mRNA levels of the various genes for those two stages of bladder cancer were compared through Welch's t-test or through Wilcoxon rank-sum test if data was not parametric (Shapiro-Wilk p-value $<0.05$ ).

Results: Decreased mRNA expression for E-cadherin in superficial compared to myoinvasive bladder was observed in 7 out of 8 datasets, with 3 of them being significant $(\mathrm{p}<0.05)$. Increased mRNA expression was observed in all other proteins for muscle-invasive cases compared to non-myoinvasive urothelial cancer: N-cadherin 8 out of 8 datasets, 5 significant; fibronectin 8 out of 8 datasets, 7 significant; Snail 3 out of 4 datasets, 3 significant; Slug 6 out of 6 datasets, 1 significant; and Twist 5 out of 6 datasets, 3 significant.

Conclusions: The expression trends of E-cadherin, N-cadherin, Slug, Snail, Fibronectin, and Twist between Infiltrating Urothelial and Superficial bladder cancer mimics what is expected of cells undergoing the Epithelial-Mesenchymal transition. Data mining is a simple way to test hypotheses before embarking in more complex tissue-based research projects.
849 PTEN Loss Is Less Frequent in African-American Compared to European-American Prostate Cancers

Fawaz Almutairi, Carlos L Morais, Stephanie Glavaris, Jessica Hicks, Deb Sundi, Angelo M De Marzo, Edward M Schaeffer, Tamara Lotan. Johns Hopkins School of Medicine, Baltimore, MD

Background: In patients of European-American (EA) descent, $E R G$ rearrangement and $P T E N$ deletion are among the most common somatic genetic alterations. PTEN loss is enriched among $E R G$-rearranged tumors and occurs secondary to $E R G$ rearrangement. Though $E R G$ rearrangement is approximately half as common among prostate cancers arising in patients of African-American (AA) descent compared to EA prostate cancers, the rate of PTEN loss among AA prostate cancers has not been studied in large cohorts. Design: We used genetically validated, clinical-grade IHC assays to assess ERG and PTEN status in prostate tumors from 1119 EA and 168 AA patients who underwent radical prostatectomy at Johns Hopkins. Race was determined by self-report. There were no significant differences in patient age, tumor grade or stage between EA and AA patients.

Results: Despite the similar clinical-pathologic characteristics in the two groups, the rate of PTEN loss was significantly lower among AA tumors compared to EA tumors (19\% vs. $35 \%$; $<0.0001)$. This lower rate of PTEN loss among AA tumors was proportionate to expected lower rate of ERG-positive tumors in AA versus EA patients $(27 \%$ vs. $50 \%$, $\mathrm{p}<0.0001)$. Thus, PTEN loss is similarly enriched among ERG-positive tumors both groups (2.7-fold in AA patients and 2.4-fold in EA patients).

Conclusions: Both PTEN loss and AA ancestry are associated with poor outcomes in prostate cancer. Surprisingly, however, PTEN loss is significantly less common among AA compared to EA tumors, even in grade- and stage-matched cohorts, suggesting that alternative molecular changes may predominate in AA tumors. The decreased rate of PTEN loss among AA tumors is proportionate to the known lower rate of ERG rearrangement in this group, suggesting that the molecular interaction of PTEN and ERG may be similar in AA and EA tumors.

850 Clinicopathologic and Immunohistochemical Characteristics of Urinary Bladder(UB) Inflammatory Myofibroblastic Tumors (IMT), Including 10 Malignant Cases

Isabel Alvarado-Cabrero, Narciso Hernandez-Toriz, Alejandra Mantilla-Morales, Blandina Hernandez-Cruz, Raquel Valencia-Cedillo. Oncology Hospital, Mexican Institute of Social Security, DF, Mexico.

Background: Inflammatory myofibroblastic proliferations of the urinary tract represent a variety of lesions ranging from benign "postoperative spindle cell nodules" and IMT to frankly malignant fibrosarcomas. Over the years, there has been considerable debate concerning the aggressivity, nomenclature and mere existences of some nosologic entities

The goal of this study was to evaluate the morphologic and immunohistochemical features of 54 cases of IMT of the UB

Design: This retrospective study was done in the department of pathology of our Institution with cases of IMT that were collected from 2000-2010.

Immunohistochemical studies were performed in all cases, using a panel of antibodies raised against: ALK-1, AE1/AE3, CAM 5.2, Desmin and SMA

Results: There were 32 males and 22 females aged 18 to 91 years (mean:59). There was a history of recent prior instrumentation in 6 cases. Signs and symptoms included hematuria, irritative or obstructive voiding symptoms. Grossly most lesions were polypoid or nodular and measured 1.2 to $21 \mathrm{~cm}(\mathrm{~m}: 6 \mathrm{~cm})$. Microscopically, most tumors (44/54) had the typical appearance of IMT ocurring in this region. Mitotic figures ranged from 0 to 15 mitoses per 10 high power fields. However, 10 cases showed some portions of classic IMT admixed with large fibrosarcomatous areas. By immunohistochemistry, $48 \%$ of cases expressed ALK-1, 96\%, AE1/AE3, 70\% CAM $5.2,50 \%$ Desmin and 20\% SMA.

Follow-up: $69 \%$ of patients had at least 1 recurrence, on the other hand all cases with fibrosarcomatous areas had $>3$ recurrences. 6 patients with IMT and fibrosarcomatous areas died of disease and 4 are alive with disease

Conclusions: Most of IMT cases have a benign course but some cases (18.5\% ) may develop a malignant transformation

851 Clinical Utility of Comparative Genomic Array Testing for Unclassified and Advanced Stage Renal Cell Carcinomas

Nicole Andeen, Xiaoyu Qu, Tatjana Antic, Scott Tykodi, Min Fang, Maria S Tretiakova. University of Washington, Seattle, WA; University of Chicago, Chicago, IL. Background: Cytogenetic analysis provides a useful adjunct to traditional categorization of renal cell carcinomas (RCC), particularly in morphologically ambiguous cases. We utilize comparative genomic array testing (CGAT) to 1) investigate unclassified RCCs and 2) identify chromosomal aberrations in metastatic clear cell RCCs associated with a worse prognosis.

Design: DNA was isolated from paraffin-embedded tissue from 22 unclassified RCCs and 11 cases of clear cell RCC with metastasis. CGAT testing was performed using the OncoScan ${ }^{\mathrm{TM}}$ assay (Affymetrix, CA); acquired data were analyzed using the Affymetrix Chromosome Analysis Suite and Nexus Express (BioDiscovery Inc, CA).

Results: Seventeen of 22 cases $(77 \%)$ of unclassified RCC were reclassified as a result of this analysis. The ambiguous cases frequently had papillary, solid or tubular architecture, densely eosinophilic cytoplasm and high nuclear grade. Incorporating cytogenetic and morphologic features, half of all unclassified cases could be classified as clear cell RCC $(-3 p ; 82 \%$ also with $+5 q)$. In the 11 non-clear cell cases, $55 \%$ could be classified as chromophobe, papillary or collecting duct RCC, or oncocytoma. 5 cases remained unclassified, which had morphologic, immunohistochemical and cytogenetic features that could not be integrated into a single diagnosis. 


\section{Unclassified RCCs, pre and post CGAT}

\begin{tabular}{|c|c|c|c|}
\hline Morphologic features/favored diagnosis & \# cases & $\begin{array}{l}\text { CGAT } \\
\text { findings }\end{array}$ & Final reclassification \\
\hline \multirow[t]{2}{*}{$\begin{array}{l}\text { Translocation RCC or Papillary RCC, type } 2(n=6 ; 55 \%) \\
\text { Extensive rhabdoid features }(n=2 ; 18 \%) \\
\text { Chromophobe RCC }(n=2 ; 18 \%) \\
\text { Hereditary leiomyomatosis RCC }(n=1 ; 9 \%)\end{array}$} & $11(50 \%)$ & $-3 p,+5 q$ & Clear cell RCC \\
\hline & $11(50 \%)$ & & Non-clear cell \\
\hline $\begin{array}{l}\text { Collecting duct-like }(n=2 ; 40 \%) \\
\text { Translocation associated } R C C(n=2 ; 40 \%) \\
\text { Epithelioid angiomyolipoma }(n=1 ; 20 \%)\end{array}$ & $5(23 \%)$ & $\begin{array}{l}\text { Non- } \\
\text { conventional }\end{array}$ & $\begin{array}{l}\text { Remained } \\
\text { unclassified }\end{array}$ \\
\hline $\begin{array}{l}\text { Succinate dehydrogenase deficient (SDH; } n=1 \text { ) } \\
\text { Papillary RCC, type } 2(n=1)\end{array}$ & $2(9 \%)$ & $-1,-2,-13$ & Chromophobe RCC \\
\hline Neuroendocrine-like or thyroid-like RCC & $1(5 \%)$ & $\begin{array}{l}+7,+17 \\
\text { without }-3 p\end{array}$ & Papillary RCC \\
\hline Papillary RCC, type 2 & $1(5 \%)$ & $-1 p-8 p,-9 p$ & $\begin{array}{l}\text { Collecting duct } \\
\text { carcinoma }\end{array}$ \\
\hline Hybrid oncocytic tumor & $1(5 \%)$ & -1 & Oncocytoma \\
\hline SDH deficient & $1(5 \%)$ & Normal & $\begin{array}{l}\text { No cytogenetic } \\
\text { abnormalities }\end{array}$ \\
\hline
\end{tabular}

A previously undescribed amplification of TFEB $(+6 \mathrm{p} 21)$ was identified in one case. Of 11 cases of clear cell RCC with metastasis, 10 had loss of chromosomal material on $3 p(91 \%)$ and $7(63 \%)$ had cytogenetic features associated with a poor prognosis (PMID: 23552698). One case of clear cell RCC with classic morphology and dense inflammation had CGAT features of papillary RCC $(+7,+17$, no $-3 \mathrm{p})$.

Conclusions: In our study, CGAT was effectively utilized to reclassify $77 \%$ of unclassified RCCs. This has practical value in our institution, where a molecular profile separating clear cell or papillary RCC from unclassified RCC influences treatment recommendations and/or clinical trial eligibility. CGAT can also be utilized for prognostication of advanced CCRCC. Our data expand the molecular understanding of difficult-to-classify RCCs.

852 Can Contemporary Pure Gleason 3+3 (ISUP Grade Group 1) Prostate Cancer Invade into the Extraprostatic Tissue or Seminal Vesicle? Blake Anderson, Aria A Razmaria, Bonnie Choy, Scott Eggener, Gladell P Paner. University of Chicago, Chicago, IL.

Background: Recent studies employing contemporary Gleason grading have demonstrated that Gleason score 6 prostate cancers lack the ability to metastasize. The goal of this study was to assess the pathologic stage following radical prostatectomy of true Gleason score 6 prostate cancers in a large institutional series.

Design: From 2003 to 2013, 3731 patients underwent robotic radical prostatectomy at a single academic institution. Out of this cohort, 38 cases were identified as being Gleason 6 prostate cancer with stage $\mathrm{pT} 3 \mathrm{a} / \mathrm{b}$ on initial pathologic review, 34 with $\mathrm{pT} 3 \mathrm{a}$ (extra-prostatic extension or EPE) and 4 with pT3b disease (seminal vesicle invasion or SVI). All cases underwent repeat pathologic evaluation by a single expert genitourinary pathologist for Gleason grade, pathological stage, extent of EPE, percent of prostate volume with tumor, percent of Gleason pattern 4 and type of Gleason pattern 4 (e.g. cribriform, fused, ill-formed, glomeruloid, variant patterns). The 2014 International Society of Urological Pathology (ISUP) modified Gleason grading criteria were applied. Results: The reviewed cases were from the years 2003 (4), 2004 (10), 2005 (9), 2006 (8), 2007 (2), 2008 (1), 2011 (2), 2012 (2), respectively. Out of the 38 reviewed cases, $34(89.5 \%)$ were upgraded to $3+4(32), 4+3$ (1) or $4+4$ (1). Of the upgraded cases, 8 $(23.5 \%)$ had only minor $(<5 \%)$ components of pattern 4 , which under the 2005 ISUP Gleason grading consensus was not established as a secondary grade. Six cases $(15.8 \%)$ were down-staged to $\mathrm{pT} 2$ because of only capsular incision being present or tumor invading into the capsule but not beyond it. Among the cohort, only 2 cases $(5.9 \%)$ with purely Gleason 6 and pT3a stage were noted. In these cases the EPE was focal. Among the re-examined cohort, no cases with pure Gleason 6 and stage pT3b were observed. Ill-formed glands were the most common type of pattern 4 present in $91.2 \%$ of upgraded cases, followed by fused $(82.4 \%)$ and cribriform (35.3\%) glands. Ductal $(5.9 \%)$ and mucinous $(2.9 \%)$ variants were rare reasons for upgrading.

Conclusions: In this large contemporary study, Gleason 6 prostate cancers never had SVI $(0 \%)$ and were only rarely associated with EPE that is only focal in extent. This supports previously reports on the non-aggressive nature of Gleason score 6 prostate cancer as well as active surveillance as an optimal management strategy for this disease.

853 CK20 and p53 Immunohistochemistry: Staining Patterns and Follow Up in Urinary Bladder Specimens with Urothelial Atypia

Javier Arias-Stella, Alpa B Shah, Nilesh S Gupta, Sean R Williamson. Henry Ford Hospital, Detroit, MI.

Background: Urinary bladder carcinoma in situ (CIS) is associated with a considerable risk of progression to invasive bladder cancer, and refractory disease may necessitate radical surgery. Therefore, distinction from reactive urothelium is critical. CK20 and p53 are among the most common immunohistochemical (IHC) antibodies used for this purpose. However, to date, most studies have investigated specimens with established benign or malignant diagnoses. In contrast, we studied the use of CK20 and p53 in bladder specimens with borderline or suspicious features for CIS and correlated with subsequent or prior cancer diagnoses.

Design: Specimens with equivocal urothelial atypia from 2008-2015 were retrieved. CK20 and p53 IHC was performed to analyze staining pattern and intensity. Staining was classified as CIS pattern (both stains yielding strong uniform labeling of the area of concern), discordant (only 1 yielding CIS pattern), indeterminate ( 1 or both yielding partial or equivocal labeling), or benign (both stains yielding a benign pattern). Prior and subsequent bladder specimen diagnoses were reviewed.

Results: A total of 69 specimens from 65 patients with equivocal atypia were retrieved. Nine $(13 \%)$ had a CIS staining pattern, $18(26 \%)$ were discordant, $31(45 \%)$ were indeterminate, and $11(16 \%)$ showed a benign staining pattern. Of the discordant specimens, 13 labeled for CK20 but not p53, whereas 5 showed the opposite. Most patients ( $\mathrm{n}=43 ; 65 \%)$ had a known history of bladder cancer, of whom 26 had recurrence with an average interval of 37 months (2-216). A subset of patients $(n=23 ; 35 \%)$ had no prior history of bladder cancer. Of these, only 1 patient with $\mathrm{CK} 20+$ staining later developed diagnostic carcinoma.

\begin{tabular}{|l|l|l|l|l|}
\hline IHC Pattern & $\begin{array}{l}\text { No History of } \\
\text { Bladder Cancer }\end{array}$ & $\begin{array}{l}\text { Subsequent } \\
\text { Cancer }\end{array}$ & $\begin{array}{l}\text { History of } \\
\text { Bladder Cancer }\end{array}$ & $\begin{array}{l}\text { Recurrence of } \\
\text { Cancer }\end{array}$ \\
\hline CIS & 2 & & 7 & 4 \\
\hline Discordant & 9 & 1 & 9 & 8 \\
\hline Indeterminate & 10 & & 21 & 7 \\
\hline Benign & 2 & & 9 & 7 \\
\hline TOTAL & 23 & & 46 & \\
\hline
\end{tabular}

Conclusions: $\mathrm{IHC}$ for $\mathrm{CK} 20$ and $\mathrm{p} 53$ is commonly used for discrimination of urothelial CIS from benign or reactive urothelium, and has been previously studied in specimens with established morphology. In our cohort of specimens with equivocal urothelial atypia, very few patients without a prior diagnosis of bladder cancer progressed to diagnostic cancer (1/23), suggesting that an inconclusive staining pattern is similar to a diagnosis of "atypia of unknown significance". Patients with a known history of bladder cancer had a substantial rate of recurrence, independent of staining pattern.

\section{Detection Gene Fusion(s) in Melanotic Xp11 Translocation Renal} Cancer by RNA-seq

Sabina Babayeva, Weihua Huang, Humayun Islam, John T Fallon, Minghao Zhong. New York Medical College, Valhalla, NY.

Background: Melanotic Xp11 translocation renal cancer (TRC) is a recently recognized rare tumor with overlapping features of PEComa, carcinoma, and melanoma. The comprehensive molecular features of this tumor have never been studied before. In current project, we would like to detect all potential gene fusions in this tumor by RNA-seq.

Design: 5 cases of TFE3 melanotic Xp11 (confirmed by FISH) were collected for RNAseq. Total RNA was extracted from FFPE tissue, using the AllPrep DNA/RNA FFPE. 100 ng RNA was applied for sequencing library preparation using the TruSeq RNA Access Library Prep Kit as per the manufacturer's protocol. Paired-end sequencing (75 bp $\times 2$ ) was performed using the MiSeq Reagent V3 Kit (150 cycles) and the MiSeq sequencing system. STAR algorithm was employed for detection of any potential TFE3 or other fusions. Bowtie2 was employed for alignment and mapping of short sequence reads to the human genome reference $\mathrm{hg} 19$ and the fusion transcript $S F P Q / P S F-T F E 3$. Integrative Genomics Viewer (IGV) was employed for data visualization

Results: We identified 2 cases with $P S F-T F E 3$ fusion, one case with $P S F-T F E 3$, TYRP1-PRKAG2, RBM25-ABCC5, CCT2-ZC3H3 fusions, one case with PSF-TFE3, $M E 1-A B C C 5, L D B 2-O L A 1, C A R S 2-B 2 M, C L E C 16 A-A T G 7$ fusions, the last case with only $S P G 7-A N K R A 2$ fusions.

Conclusions: This is the $1^{\text {st }}$ RNA-seq study of melanotic Xp11 Translocation Renal Cancer. We confirmed the existence of PSF-TFE3 fusion in this type of tumor. In addition, other gene fusions have also been identified. Especially, $A B C C 5$ gene fusion may be a novel recurrent gene fusion in melanotic Xp11 Translocation Renal Cancer.

\section{Does Multifocality Matter? An Evidence Based Study of Multifocal} Renal Carcinomas of Clear Cell Type

Burak Bahar, Lu Wang, Nicole Andeen, Funda Vakar-Lopez, Maria S Tretiakova, Neriman Gokden, Tatjana Antic, Ming Zhou, Ximing Yang, Roberto Gamez, Stefan Pambuccian, Maria M Picken, Eva M Wojcik, Guliz A Barkan. Loyola University Medical Center, Maywood, IL; Norhwestern University Feinberg School of Medicine, Chicago, IL; University of Washington, Seattle, WA; University of Arkansas, Little Rock, AR; University of Chicago, Chicago, IL; NYU Medical Center Tisch Hospital, New York, NY.

Background: The International Society of Urologic Pathology provided the recommendations on the reporting of renal neoplasms, however, a consensus was not reached on the reporting of multifocal tumors. Our aim was to investigate if there is a difference in survival rate between unilateral multifocal and unifocal renal tumors. Design: An electronic medical record search was conducted for unilateral synchronous multifocal carcinomas of renal origin from January 2004 to September 2015 in six institutions. Tumor type was limited to renal cell carcinoma, clear cell type (CCRCC), and size of the secondary tumor focus was limited to $>1 \mathrm{~cm}$. Grade and size matched patients with a single mass lesion diagnosed as CCRCC were randomly selected for statistical comparison. Clinical variables evaluated in this study included age, gender, tumor grade, multifocality, capsule invasion and tumor size(s). There was a minimum 3 -year follow up and the end point was overall survival. Welch two sample t-test was used for comparison of data. Cox proportional-hazard regression analysis was performed to assess the effect of variables on survival rate.

Results: The mean survival of patients with multifocal CCRCC $(\mathrm{n}=68)$ was similar to that of unifocal CCRCC cases $(\mathrm{n}=64)(P=0.317)$. In patients with unifocal tumors, greatest tumor dimension correlated with time-to-death (HR: $1.18 ; P=0.011$ ), but in patients with multifocal tumors the largest tumor's greatest dimension did not show significance $(P=0.064)$. In patients with multifocal tumors, evaluation of the second tumor's greatest dimension did not show significance with time-to-death $(P=0.165)$. Also, in patients with multifocal tumors, evaluation of the addition of first and second tumor's greatest dimension did not show significance with time-to-death $(P=0.672)$. Conclusions: In our pilot study we found no significant difference in survival between unilateral multifocal and unifocal CCRCCs suggesting that measuring secondary tumor 
nodules has no added value. In multifocal tumor cases, neither evaluation of the second tumor's greatest dimension nor evaluation of the sum of the greatest dimension of the first and second tumors showed significance with time-to-death.

856 The Ratio of CD8 to Treg Tumor Infiltrating Lymphocytes Is Associated with Neoadjuvant Cisplatin Based Chemotherapy Response in Patients with Muscle Invasive Urothelial Carcinomas of the Bladder Alexander Baras, Charles Drake, Jen-Jane Liu, Nilay Gandhi, Max Kates, Mohamed Hoque, Alan Meeker, Noah M Hahn, Mark Schoenberg, Janis Taube, Trinity J Bivalacqua, George J Netto. Johns Hopkins, Baltimore, MD; Montefiore Medical Center, Bronx, NY.

Background: Randomized controlled trials of platinum-based neoadjuvant chemotherapy (NAC) for muscle invasive bladder cancer (MIBC) have shown that patients who achieve a pathologic response to NAC exhibit 5 yr cancer specific survival (CSS) of $\sim 80-90 \%$ while NAC resistant cases exhibit 5 yr CSS of $\sim 30-40 \%$. This highlight the need to predict who will benefit from conventional NAC and the need for plausible alternatives. In this study, we aim to characterize how the immunological microenvironment in bladder cancer is related to response to conventional chemotherapy and to identify related plausible alternatives.

Design: The pre-treatment biopsy tissues from a cohort of 41 patients with MIBC who were treated with NAC were incorporated in tissue microarray format and subsequently stained these for PD-L1, CD8, and FOXP3. Percentage of PD-L1 positive tumor cells was measured. Additionally, overall along with CD8 and Treg specific tumor infiltrating lymphocytes (TIL) densities were measured.

Results: TIL density was associated with tumor PD-L1 expression, consistent with the notion of adaptive immune resistance in bladder cancer. Tumor PD-L1 expression was not a significant predictor of response; neither was the CD8 nor Treg TIL density. Intriguingly though, the ratio of CD8 to Treg TIL densities was strongly associated with response, supporting the hypothesis that the immune system plays a role in the response of bladder cancer to chemotherapy.
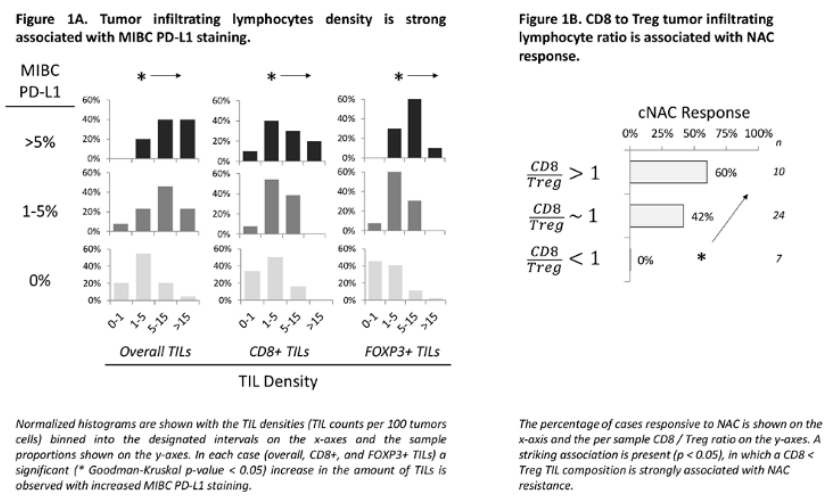

Conclusions: While similar relationships have been found in other cancers, to our knowledge this is the first such report in bladder cancer showing that the CD8 to Treg TIL density in the pre-treatment tissues is assicated with NAC response. These findings warrant further investigations to both better characterize this association in larger cohorts and begin to elucidate the underlying mechanism(s) of this phenomenon. The findings raise the possibility of utilizing anti-PD-L1/PD1 therapy in conjunction with or as an alternative to conventional NAC in the appropriate clinical setting.

857 An Immunohistochemical Study of the Molecular Subtypes of Combined Urinary Bladder Urothelial and Small Cell Carcinoma

Mary Barrett, Pamela Unger, Jerome Jean-Gilles, Nisha Patel, Qi Yang, Loralee McMahon, Guang-Qian Xiao. University of Rochester Medical Center, Rochester, NY; Lenox Hill Hospital - LIJ Northshore Health System, New York, NY

Background: Recent studies have shown that urinary bladder cancer can be classified into three molecular subtypes (luminal, p53-like and basal), which bear prognostic and therapeutic significance. Small cell carcinoma (SmCC) of the bladder is rare and frequently coexists with conventional urothelial carcinoma (UC). The immunohistochemical (IHC) profile of combined bladder UC and SmCC has not yet been studied. The aim of this investigation is to elucidate this IHC profile and compare the IHC of combined tumors to the IHC of the molecular subtypes of bladder cancer. Design: A total of 18 combined UCs and SmCCs were studied. The amount of SmCC in the tumor ranged from $5 \%$ to $95 \%$. The UC was present as carcinoma in situ (CIS), invasive carcinoma, or both. Immunohistochemical markers used included GATA3, CK20, HER2, p53, Uroplakin III, CK5, CK14 and CD138. UC components were subclassified into luminal and basal types based on their IHC phenotypes as defined by previous molecular studies.

Results: Among the UCs of the 18 cases, 12 (67\%) were luminal-type (CK20+/ GATA3+/HER2+), of which 10 (83\%) had mutated p53 (Tp53), 2 (17\%) had wild type p53 (Wp53), and $10(83 \%)$ had moderate to strong HER2 expression. Four (22\%) were basal-type UCs (CK5+/CK14+), of which $3(75 \%)$ had Tp53 and 1 (25\%) had Wp53. Two (11\%) were Wp53 UCs and did not express other markers. The UC markers were mostly lost in the coexisting SmCCs (6 GATA3+, 2 CK20+, 0 HER2+, 2 CK5+, 0 $\mathrm{CK} 14+$ ), except for $\mathrm{p} 53$, in which the SmCC preserved the same pattern as the UC (13 Tp53 and $5 \mathrm{Wp} 53$ ). None of the tumors expressed Uroplakin III, and CD138 was expressed in majority of the UCs $(17 / 18,94 \%)$ as well as SmCCs $(11 / 18,61 \%)$.
Conclusions: $\mathrm{SmCC}$ was much more commonly associated with luminal type than basal type UC. SmCC also tended to lose the coexisting UC markers, except for p53. The 5 cases with wild type p 53 may represent the p53-like bladder cancer subtype; however, further p53 gene expression study would be helpful for confirmation. Since the luminal type of bladder cancer is reported to have better prognosis and good response to cisplatin-based therapy as well as possible HER2 targeted treatment (due to its high expression of HER2), recognition of this association may be useful in guiding clinical management of these combined bladder cancers.

858 TCF3: A Sensitive Marker to Differentiate Seminomatous from Non-Seminomatous Germ Cell Tumors

Mary Barrett, Asif Shahab, Nisha Patel, Qi Yang, Loralee McMahon, Guang-Qian Xiao, Faqian Li. University of Rochester Medical Center, Rochester, NY; University of Minnesota, Minneapolis, MN.

Background: For management purposes, testicular germ cell tumors (GCT) can be classified into seminomatous and non-seminomatous types. Following surgical resection, seminomas are generally treated with low-dose radiation, whereas non-seminomatous GCTs are treated with chemotherapy if needed. The difference in treatment regimens makes a correct diagnosis imperative. Although distinction between these two can often be achieved by microscopic morphology alone, ancillary tests may be needed in challenging cases. Currently, no sensitive biomarkers are available for this purpose. T-cell factor 3 (TCF3), a component of the Wnt signaling pathway in embryonic stem cells, plays an important role in control of pluripotent self-renewal and lineage specification. Here we examine the immunohistochemical expression and diagnostic utility of TCF3 in GCTs.

Design: Fifty cases of testicular GCTs were collected: 23 seminomas, 6 embryonal carcinomas, 1 teratoma, 1 choriocarcinoma, and 19 mixed GCTs. The components of the mixed GCTs were seminoma $(n=3)$, embryonal carcinoma $(n=18)$, yolk sac tumor $(\mathrm{n}=9)$, teratoma $(\mathrm{n}=15)$, and choriocarcinoma $(\mathrm{n}=4)$. On immunohistochemistry, only nuclear staining was considered positive. Staining was graded as negative $(<5 \%$ of tumor cells stained), $1+(5-25 \%$ positive), $2+(26-50 \%)$, and $3+(>50 \%)$.

Results: All non-seminomatous components $(\mathrm{n}=54)$ exhibited positive nuclear expression of TCF3 $(54 / 54 ; 100 \%)$. The strength of positivity ranged from $1+$ to $3+$, with the majority of cases showing $3+$ staining $(33 / 54 ; 61 \%)$. In contrast, no TCF3 expression was detected in the majority of seminomatous tumor components $(24 / 26$; $92 \%)$. Two seminomas $(2 / 26 ; 8 \%)$ exhibited weak (1+) and focal positivity $(5 \%$ and $5-10 \%$ of cells, respectively,) for TCF3.

Conclusions: TCF3 is a highly sensitive marker for separating seminomatous from non-seminomatous GCTs, and may aid in the distinction of these two therapeutically different entities, benefiting clinical patient management.

859 Human Papillomavirus in Urothelial Carcinoma In-Situ: Does It Have a Role in Urothelial Carcinogenesis?

Sheldon I Bastacky, Dinesh Pradhan, Somak Roy, Rajiv Dhir. UPMC, Pittsburgh, PA. Background: Urothelial carcinoma in situ (UCIS) is recognized to overexpress p16 ink4a. HPV- associoated squamous pharyngeal and genital tract neoplasms frequently overexpress p 16 protein. We recently identified a bladder biopsy with UCIS exhibiting diffuse p 16 and p53 immunoreactivity and integrated high risk HPV detected by in situ hybridization (ISH). The aim of this study is to evaluate the prevalence of human papillomavirus (HPV) in p16-positive UCIS

Design: A computer search of the CoPath pathology database to retrieve all bladder UCIS cases (biopsies and TURBTs) diagnosed between $1 / 2013$ and $8 / 2015$ was performed. A p16 immunostain was performed on all cases; high-risk (HPV 16, 18) HPV ISH was done on all UCIS with diffuse p16 immunoreactivity.

Results: Of the 67 UCIS cases, 28 (41.8\%), 25 (37.3\%), and 14 (20.9\%) showed strong / diffuse, patchy, and negative p16 immunoreactivity, respectively. HPV ISH performed on the 28 p 16 diffusely positive UCIS cases revealed integrated punctate nuclear high-risk HPV DNA in urothelial carcinoma $(\mathrm{n}=2)$ and equivocal staining (punctate nuclear positivity with extensive background staining $(\mathrm{n}=3)$. The remaining 23 UCIS cases were HPV-negative. Mean (range) age was 70.7 y (44-94 years) with a male predominance (20.9:1). The HPV-positive cases were both male with relatively younger age (57 and 82 years).

Conclusions: Overexpression of p16 in UCIS may be multifactorial, with HPV association in a small subset of UCIS (7.2\%), suggesting an etiologic role for HPV in urothelial carcinogenesis in a minor subset of urothelial bladder cancer.

860 Clear Cell Urothelial Carcinoma: A Clinicopathological Study of Nine Cases and a Review of Literature

Justin Bateman, Jason K Wasserman, Trevor A Flood, Bojana Djordjevic, Eric C Belanger, Kien T Mai. The Ottawa Hospital and University of Ottawa, Ottawa, ON, Canada.

Background: Clear cell urothelial carcinoma (CCUC) is a rare variant of urothelial carcinoma (UC) and its clinical significance has not been well elucidated. We reviewed the clinicopathological characteristics of CCUC cases at our institution, and compared the findings to cases of CCUC reported in the literature.

Design: Consecutive cases of UC over a period of five years at our institution were reviewed to identify cases of CCUC. Histological characteristics recorded included: proportion of tumor cells with clear cell change (CCC), the overall pattern of tumor growth, patterns of the intra-urothelial and invasive tumor components, tumor stage, and the original diagnosis rendered. Clinical information including patient sex, age, and outcome was abstracted. A review of the medical literature was conducted for reported cases of CCUC. 
Results: Of the total 826 cases of UC, including 352 invasive UC, there were a total of 9 CCUC cases. They were high grade and exhibited a distinctive histopathological pattern with clear cytoplasm and prominent cytoplasmic membranes. Clear cell component accounted for $30-90 \%$ of invasive UC in these 9 cases. The intra-urothelial component was flat and inverted, with or without minor papillary structures, and intra-urothelial CCUC was seen in 3 cases. Of all the non-CCUC cases reviewed, at least $50 \%$ (noninvasive or invasive UC) showed focal areas of CCC that accounted for less than $5 \%$ of the neoplastic cells. Of the nine case, one was originally diagnosed as a nested variant of UC. Another 3 cases that showed high grade nuclear atypia with tumoral necrosis, including sarcomatoid change in one case, were classified as usual type of UC. A review of the medical literature revealed 28 cases of CCUC. A comparison of our series versus the 28 reported cases of CCUC showed: a) male predominance with sex ratio of M:F=9:0 vs. $4: 1$ and mean age of $70 \pm 8$ vs. $67 \pm 9$, b) clinical stage pT3-4 in 7/9 $(79 \%)$ vs. $5 / 8(63 \%)$, and c) patient death or survival with disease in a high proportion of cases, $3 / 9(33 \%)$ vs. $4 / 12(33 \%)$, respectively.

Conclusions: Our cohort of CCUCs represents the largest case series to date. CCUC can be distinguished from non-CCUC by the extensive CCC in more than $30 \%$ of cells, and is often under-diagnosed. This variant is associated with male predominance, an aggressive clinical course, with rapid progression to muscle invasion, metastasis, and death.

861 Clear Cell Urothelial Carcinoma: Immunohistochemical and Topographic Studies Are Suggestive of Mesonephric Differentiation

Justin Bateman, Jason K Wasserman, Trevor A Flood, Eric C Belanger, Bojana Djordjevic, Kien T Mai. The Ottawa Hospital and University of Ottawa, Ottawa, ON, Canada.

Background: Clear cell urothelial carcinoma (CCUC) is a rare variant of urothelial carcinoma (UC) with a male predominance. Despite some similarities between CCUC and clear cell adenocarcinoma of the lower urinary tract, CCUC is not thought to be of mesonephric duct origin. In this study we sought to characterize the immunohistochemical profile of CCUC. We further hypothesized that immunohistochemical evidence of mesonephric differentiation may be found in normal urothelium.

Design: Nine consecutive cases of CCUCs from our institution were studied with the following immunohistochemical markers: CK7, CK20, CK5, CD44 and PAX8. Specific location in the urinary tract was also recorded. Comparisons were made to cases reported in the medical literature. PAX8 immunohistochemistry was also applied to normal urothelium from 20 ureters and 20 bladders (including trigone, lateral, and anterior walls ) derived from non-CCUC specimens.

Results: CCUCs exhibited reactivity for GATA3/CK7 ( $n=9 / 9)$ and CK20 ( $=5 / 9)$, as is commonly reported in the literature. In addition, tumor cells were reactive for basal cell markers CK5/CD44 (n=8/9) and PAX8 (very focal to extensive; $n=5 / 9)$. All CCUCs were located in the ureter $(n=1 / 9)$, trigone $(n=6 / 9)$ or in the vicinity of the trigone $(\mathrm{n}=2 / 9)$. Of the 28 reported cases in the literature, ten cases were informative for tumor location in the urinary tract. Seven cases were in the trigone and three were in the lateral walls. In normal urothelia, PAX8 reactivity was seen in 8/20 sections of ureter and 7/20 sections of bladder ( 5 of trigone, 2 of lateral wall, and none of anterior wall). PAX8 reactivity was seen in clear and non-clear urothelial cells.

Conclusions: For the first time, we demonstrate mesonephric differentiation in CCUC based on its immunohistochemical profile and location in the ureter and the trigone. Furthermore, we show an element of mesonephric differentiation in normal urothelium at these sites. As such, CCUC appears to be an entity distinct from the conventional urothelial carcinoma, which may derive from a different cell precursor.

862 Quantitative Assessment of Perineural Invasion and Percentage of Sites with 3+4=7 Carcinoma Predicts Upstaging in Intermediate Risk Prostate Cancer

Justin Bateman, Susan J Robertson, Nicola Schieda, Daniel T Keefe, Eric C Belanger, Kien T Mai, Rodney H Breau, Chris Morash, Trevor A Flood. The Ottawa Hospital, Ottawa, ON, Canada; The University of Ottawa, Ottawa, ON, Canada.

Background: Selected men with Gleason score $3+4=7$ prostate cancer (PCa) detected at transrectal ultrasound (TRUS)-guided biopsy may be considered candidates for active surveillance (AS); however, a significant proportion may harbor more aggressive disease. This study assesses if quantitative analysis of biopsies with perineural invasion (PNI) and percentage of sites involved with Gleason Score $3+4=7$ predicts upstaging and/or upgrading after radical prostatectomy (RP).

Design: One hundred fifty-four patients were identified who underwent TRUS-guided biopsy with Gleason score 3+4=7 PCa and subsequent RP between January 2010 and June 2015. The presence or absence of PNI, the percentage of cores with PNI (\%PNI) and percentage of biopsy sites ( 10 sites; using standard extended sextant 12-core scheme) containing Gleason score $3+4=7(\% 3+4=7)$ were recorded. The Gleason score and stage (presence or absence of organ-confined disease $[\mathrm{OCD}]$ ) were retrieved from RP reports. Data were compared using multi-variate regression and ROC curve analysis. Patients were evaluated for AS candidacy using the University of Toronto ( $\mathrm{U}$ of $\mathrm{T}$ ) guidelines and Royal Marsden Criteria (RMC).

Results: Non-OCD was identified in $44.8 \%(69 / 154)$ of patients and $28.6 \%(44 / 154)$ of patients were upgraded after RP. $\% 3+4=7$ was associated with non-OCD $(27.0 \pm$ 18.5 vs. $20.6 \pm 14.4 \%,[\mathrm{p}=0.02])$ but not upgrading $(\mathrm{p}=0.09)$. Presence of PNI was not associated with upgrading $(\mathrm{p}=0.8)$ but was weakly associated with non-OCD $(12.1 \pm$ 18.5 vs. $7.1 \pm 15.2 \%$, $[\mathrm{p}=0.05]$. There was a correlation between $\%$ PNI and non-OCD $(\mathrm{r}=0.18, \mathrm{p}=.03)$. Area under the ROC curve for $\% 3+4=7$ and $\%$ PNI for prediction of non-OCD were: ROC 0.59 (CI $0.50-0.68)$ and 0.59 (CI 0.51-0.67) respectively. Optimal thresholds of $>36 \% 3+4=7$ and $>20 \%$ PNI yielded sensitivity/specificity of $31.9 / 87.2 \%$ and $30.9 / 82.4 \%$ respectively. Applying these additional thresholds to those patients eligible for AS would have excluded 28\% (11/39) of patients from the RMC group who were ultimately upstaged after RP. No patients would have been excluded from the $\mathrm{U}$ of $\mathrm{T}$ group.

Conclusions: Quantitative reporting of percentage of core biopsies with PNI and sites with Gleason score $3+4=7$ were predictive of non-OCD after RP. These additional criteria may help optimize enrolment into AS protocols for patients with intermediate risk prostate cancer.

863 Validation of Reporting Percentage High Grade Prostate Cancer in 988 Conservatively Treated Patients with Long Term Outcome

Daniel Berney, Gabrielle Fisher, Bernard North, Henrik Moller, Peter Scardino, Jack Cuzick, Luis Beltran. Queen Mary University of London, London, United Kingdom; Memorial Sloan-Kettering Cancer Center, New York, NY; Kings College London, London, United Kingdom.

Background: The Chicago ISUP conference in 2014 approved 5 Grade Groups for prostate cancer and has recommended that the percentage of high grade $(\% \mathrm{HG})$ Gleason patterns 4 and 5 should be quantitated. However there is debate as to whether the biopsy with the 'worst' $\% \mathrm{HG}$ disease or an 'overall' $\% \mathrm{HG}$ be reported. This has not been assesed in a cohort using prostate cancer death as an outcome measure.

Design: Cases of prostate cancer were identified from three cancer registries in the UK from men with clinically localized prostate cancer diagnosed by needle biopsy from 1990-2003. The endpoint was prostate cancer death. Clinical variables included PSA, age, clinical stage, and disease extent. Patients treated radically within 6 months, those with objective evidence of metastases or who had prior hormone therapy were excluded. Follow up was through cancer registries up until 2012. Deaths were divided into those from prostate cancer and those from other causes, according to WHO criteria. 988 biopsy cases were centrally reviewed by two uropathologists using criteria agreed at the Chicago ISUP conference in 2014. Cores were given individual Gleason scores and Grade Groups and a percentage of each Gleason grade given for each core. The " worst' $\% \mathrm{HG}$ disease seen in a biopsy series was calculated as well as the 'overall' $\% \mathrm{HG}$ disease. The hazard ratio for a $10 \%$ change in overall HG proportion was calculated for the 'overall' $\% \mathrm{HG}$ and 'worst' $\% \mathrm{HG}$ disease.

Results: The percentage of HG disease over all cores was highly significant with an $\mathrm{HR}=1.238$ for every $10 \%$ increase (CI 1.186-1.292, $\mathrm{p}<2 \times 10^{-16}$ ). This was extremely similar to the percentage of HG disease seen in the worst core. (HR=1.228, CI 1.176$\left.1.283, \mathrm{p}<2 \times 10^{-16}\right)$.

\%HG for worst core $v 10$ year survival

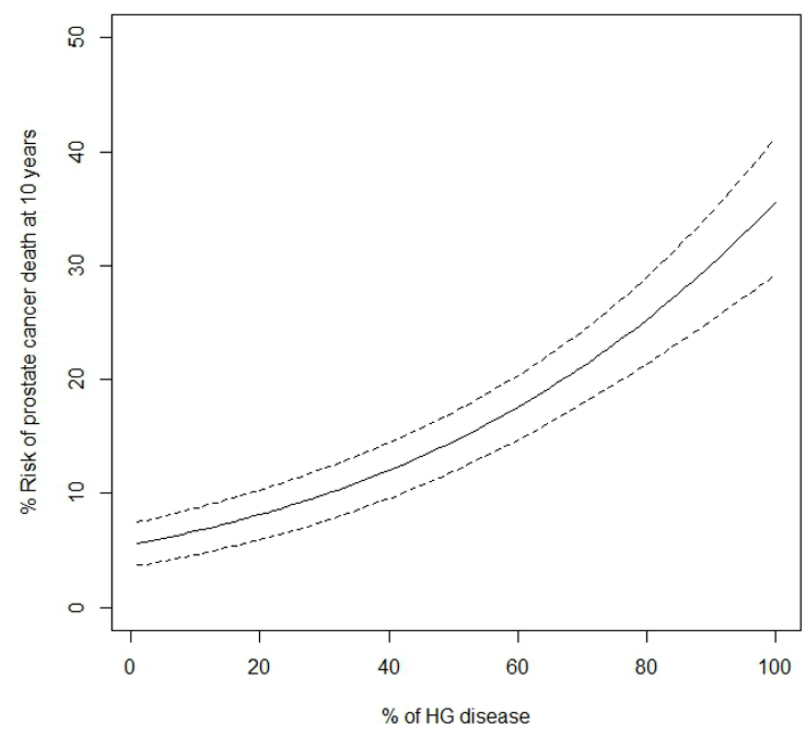

Conclusions: These data validate the use of $\% \mathrm{HG}$ disease to predict prostate cancer death. Utilisation may enable more sophisticated entry into future clinical trials based on this as well as Grade Group, such as entry into active surveillance programmes for patients of Grade Group 2 but a low $\% \mathrm{HG}$ disease. As both worst and overall $\% \mathrm{HG}$ disease are powerful predictors of outcome, either could be chosen to provide prognostic information.

864 PTEN Expression in Mucinous Prostatic Adenocarcinoma and Prostatic Adenocarcinoma with Mucinous Features: A Multi-Institutional Study of 92 Cases

Elizabeth Bertsch, Cristina Magi-Galluzzi, Liang Cheng, Adeboye O Osunkoya. Emory University School of Medicine, Atlanta, GA; The Cleveland Clinic, Cleveland, OH; Indiana University School of Medicine, Indianapolis, IN.

Background: Mucinous prostatic adenocarcinoma (MPAC) and prostatic adenocarcinoma with mucinous features (PACMF) are rare entities. Recent studies have demonstrated that most patients with MPAC have a better prognosis than those with conventional prostatic adenocarcinoma (PAC). However, it remains unclear why these tumors have a relatively good prognosis. Loss of PTEN expression, a tumor 
suppressor, is known to occur in aggressive high grade PAC, but its expression in MPAC and PACMF has not been well characterized. In this study, we sought to analyze PTEN expression in both MPAC and PACMF.

Design: A search through the Urologic Pathology files of three major academic institutions was performed for cases of MPAC and PACMF. Based on strict criteria, MPAC was selected from radical prostatectomy specimens with $25 \%$ or more of the tumor composed of extraluminal mucin. PACMF was selected from radical prostatectomy and needle core biopsy specimens. Only cases with available tissue blocks were selected. Immunohistochemical (IHC) stains were performed for PTEN. Adjacent benign glands were used as an internal control. Hematoxylin and eosin stained slides were examined and PTEN expression in matched IHC slides were scored as positive or negative.

Results: Ninety two cases of MPAC ( 34 cases) and PACMF ( 58 cases) were identified. PTEN expression was positive (retained) in 79/92 (86\%) cases; 13/92 (14\%) cases were negative for PTEN expression in the foci of MPAC (4/13 cases) and PACMF (9/13 cases). In all 92 cases, PTEN expression was retained in benign prostate glands. Conclusions: The majority of cases of MPAC and PACMF are positive for PTEN, in keeping with the fact that these tumors are known to be less aggressive than conventional PCA. The fewer PTEN negative MPAC cases compared to PACMF cases further confirms the rationale behind the $25 \%$ extraluminal mucinous component cutoff for the diagnosis of these entities. It is highly conceivable that the subset of PTEN negative tumors may still have a relatively good prognosis due to a different/PTEN independent protective pathway or mechanism.

865 Comparison of PTEN Expression in Mucinous Prostatic Adenocarcinoma, Prostatic Adenocarcinoma with Mucinous Features and Adjacent Conventional Prostatic Adenocarcinoma: Potential Evidence of Clonality

Elizabeth Bertsch, Cristina Magi-Galluzzi, Liang Cheng, Adeboye O Osunkoya. Emory University School of Medicine, Atlanta, GA; The Cleveland Clinic, Cleveland, $\mathrm{OH}$; Indiana University School of Medicine, Indianapolis, IN.

Background: Mucinous prostatic adenocarcinoma (MPCa) is a rare variant of prostatic adenocarcinoma (PCa) that has recently been shown to have a relatively good prognosis. It remains debatable whether MPCa or prostatic adenocarcinoma with mucinous features (PCaMF) and adjacent conventional PCa are clonal. It is well known that PTEN gene deletion occurs in a subset of patients with aggressive PCa. However, there is little data in the literature regarding the comparison of PTEN expression among MPCa, PCaMF and adjacent conventional $\mathrm{PCa}$.

Design: A search was made through a multi-institutional database for cases of MPCa and PCaMF. MPCa was defined as prostatectomy specimens with $25 \%$ or more of PCa composed of extraluminal/extravasated mucin. Other cases with less extraluminal/ extravasated mucin were classified as PCaMF. Only cases with tissue blocks were selected for analysis and only sections with adjacent conventional PCa were utilized. Immunohistochemical stains for PTEN were performed and the expression in MPCa, $\mathrm{PCaMF}$ and adjacent conventional PCa was scored as positive or negative. Adjacent benign glands were used as internal control.

Results: Ninety two cases of MPCa and PCaMF were identified. PTEN expression was positive (retained) in 79/92 (86\%) cases of MPCa and PCaMF. Thirteen (14\%) cases were negative for PTEN expression. PTEN expression was positive in 61/92 (66\%) of adjacent conventional PCa; 31 (34\%) cases showed loss of PTEN expression. Comparing positive MPCa and PCaMF cases to the adjacent conventional $\mathrm{PCa}, 60 / 79$ cases $(76 \%)$ had concordant expression of PTEN with the adjacent conventional PCa. Twelve of the $13(92 \%)$ PTEN negative MPCa and PCaMF cases were concordant with loss of PTEN expression in the adjacent conventional PCa. In all 92 cases, PTEN expression was retained in benign prostate glands.

Conclusions: In a significant number of cases, the expression profiles (PTEN positive or negative) of MPCa and PCaMF are similar to the adjacent conventional PCa. This correlation implies that MPCa, PCaMF and adjacent conventional PCa are likely clonal from a molecular standpoint in the majority of cases. Direction for further study includes additional biomarker analysis of the minor subset of discordant cases which would likely lead to more insight into the pathogenesis of these rare tumors.

\section{Is Zonal Distribution of Index Tumor Different in Radical} Prostatectomies Comparing Whites vs African-Brazilians?

Athanase Billis, Leandro LL Freitas, Larissa BE Costa, Camila M de Angelis, Kelson $R$ Carvalho, Ruana M Rocha, Lucas QA Bastos, Gabriel LP Oliveira, Marcel A Asato, Karina S Araujo, Daniele M Losada, Amanda P Herculiani. School of Medical Sciences, State University of Campinas (Unicamp), Campinas, SP, Brazil.

Background: Prostate cancer may be biologically more aggressive among AfricanAmericans. There is also evidence that index tumor is located predominantly in the anterior aspect of the gland in African-Americans. This anterior location is also a more difficult site to sample by biopsy. The aim of this study is to find any difference in zonal distribution of index tumor as well as adverse clinicopathological findings in African-Brazilians.

Design: From a total of 496 consecutive patients submitted to radical prostatectomy, 399 $(80.4 \%)$ were Whites and $97(19.6 \%)$ were African-Brazilians. Surgical specimens were completely step sectioned. A mean of 32 paraffin blocks was processed. Each transverse section of the prostate was subdivided into 2 anterolateral and 2 posterolateral quadrants. Tumor extent was evaluated by a previously described semiquantitative point count method. Using this method the tumor area is mapped on each quadrant examined and the index tumor located as predominantly anterior (anterolateral quadrants), posterior (posterolateral quadrants), basal, apical, at left, and at right. Other clinicopathologic findings included: age, clinical staging, preoperative PSA, weight of the prostate, PSA density, nodular hyperplasia, tumor extent, needle Gleason score, surgical specimen
Gleason score, positive surgical margins, extraprostatic extension, seminal vesicle invasion, positive lymph nodes, and time to biochemical recurrence using the KaplanMeier product limit analysis.

Results: There was no significant difference between Whites and African-Brazilians comparing the location of index tumor (anterior vs posterior, $\mathrm{p}=0.764$; basal vs apical, $\mathrm{p}=0.956$; at left vs at right, $\mathrm{p}=0.779$ ). The mean preoperative PSA was 9.04 (median 7.6) and 10.85 (median 8.9) $(\mathrm{p}=0.025)$ and the mean PSA density 0.31 (median 0.21 ) and 0.32 (median 0.24) $(\mathrm{p}=0.024)$ for Whites and African-Brazilians, respectively. No other clinicopathologic finding was significantly different including time to biochemical recurrence after surgery $(\log -\mathrm{rank}, \mathrm{p}=0.393)$.

Conclusions: Our study did not show any significant difference in zonal location of prostate cancer comparing Whites and African-Brazilians as well as any clinicopathologic adverse finding in the latter. One possible explanation may be the fact that in contrast to the bifurcated United States model, where Blacks and Whites are clearly separate groups, African-Brazilians due to miscegenation, include a large intermediate category of mulattos.

867 Does Zonal Distribution of the Index Tumor Influence Prognostic Factors in Radical Prostatectomies?

Athanase Billis, Leandro LL Freitas, Larissa BE Costa, Camila M de Angelis, Kelson $R$ Carvalho, Ruana M Rocha, Lucas QA Bastos, Gabriel LP Oliveira, Marcel A Asato, Karina S Araujo, Daniele M Losada, Amanda P Herculiani. School of Medical Sciences, State University of Campinas (Unicamp), Campinas, SP, Brazil.

Background: There are well established clinical and pathological predictors of biochemical recurrence after radical prostatectomy, including preoperative serum PSA, pathological tumor stage, Gleason histological grade, and positive surgical margins. Other features are controversial predictors, including age, race and tumor extent. The aim of this study was to find any influence on prognostic factors of zonal distribution of the index tumor (anterior vs posterior; basal vs apical; and, left vs right).

Design: The study was based on 499 consecutive patients submitted to radical prostatectomy. Surgical specimens were completely step sectioned. A mean of 32 paraffin blocks was processed. Each transverse section of the prostate was subdivided into 2 anterolateral and 2 posterolateral quadrants. Tumor extent was evaluated by a previously described semiquantitative point count method. Using this method the tumor area is mapped on each quadrant examined and the index tumor located as predominantly anterior (anterolateral quadrants), posterior (posterolateral quadrants), basal, apical, at left, and at right. The clinicopathologic findings included: age, clinical staging, preoperative PSA, weight of the prostate, PSA density, nodular hyperplasia, tumor extent, needle Gleason score, surgical specimen Gleason score, positive surgical margins, extraprostatic extension, seminal vesicle invasion, positive lymph nodes, and time to biochemical recurrence using the Kaplan-Meier product limit analysis.

Results: Posteriorly located index tumors had significantly higher mean preoperative PSA $(p=0.050)$, were more extensive $(p=0.040)$, had higher needle $(p=0.007)$ and pathologic $(\mathrm{p}<0.001)$ Gleason score, and higher frequency of lymph node metastases $(p=0.040)$. Index tumors located predominantly in the base of the prostate had significantly higher preoperative PSA $(p=0.047)$, higher frequency of extraprostatic extension $(\mathrm{p}=0.012)$, higher frequency of seminal vesicle invasion $(\mathrm{p}<0.001)$, and shorter time to biochemical recurrence after radical prostatectomy ( $\log \operatorname{Rank}, \mathrm{p}=0.002)$. Index tumors on the right side had significantly higher preoperative PSA $(\mathrm{p}=0.028)$ and higher prostate weight $(\mathrm{p}=0.017)$.

Conclusions: Index tumor location is a prognostic factor in radical prostatectomies. The location of an index tumor posteriorly and at the base of the prostate has a higher probability to be an adverse prognostic factor.

868 Morphological and Molecular Pathway-Based Analysis of Gleason Score 7 Prostate Cancer Using a 17-Gene Expression Assay Michael J Bonham, Debbie McCullough, Ruixiao Lu, John P Bennett, Phillip G Febbo, Athanasios $C$ Tsiatis. Genomic Health Inc., Redwood City, CA.

Background: Adverse outcomes associated with Gleason score 7 (GS7) prostate biopsies have been correlated with increasing amounts of Gleason pattern 4 (GP4) and certain morphologic subtypes. The genomic prostate score (GPS) is a 17-gene biopsybased RT-PCR assay analytically and clinically validated as an independent predictor of adverse pathology (AP) at prostatectomy and biochemical recurrence (BCR) (Eur. Urol 68(1):123-31, 2015). The GPS comprises genes from four gene pathways (androgen signaling, stromal response, cellular organization, and proliferation) that together were shown to discriminate cancer aggressiveness beyond individual gene pathways. Design: 5,000 prostate biopsies submitted for a GPS were centrally reviewed for GS, $\% G P 4$, and GP4 morphologic subtype. The GPS assay was performed and individual gene pathway scores were examined along with the composite GPS for all cases with GS7.

Results: $23 \%(n=1143)$ of biopsies were GS7, $20 \%(n=1005)$ were GP3 $+4(<10 \%$ GP4: $64 \%, 10-25 \% \mathrm{GP} 4: 20 \%$, and $26-50 \% \mathrm{GP} 4: 16 \%)$ and $3 \%(\mathrm{n}=138)$ were GP4+3. Poorly formed glands (PFG) was the predominant morphology $(54 \%, n=619)$, followed by fused glands $(\mathrm{FG})(24 \%, \mathrm{n}=270)$, cribriform $(19 \%, \mathrm{n}=214)$, and glomeruloid $(3 \%, \mathrm{n}=40)$. The median GPS for all GS7 biopsies was 31 (IQR 23-41). Cribriform morphology had the highest median GPS 34 (IQR 26-44), followed by PFG 32(IQR 24-41), FG 30 (IQR 22-40), and glomeruloid 25(IQR 20-32). The median GPS values were 22 (IQR 16-29), 29 (IQR 22-38), 33 (IQR 26-43), 35 (IQR 27-46), and 37 (IQR 27-47) for men with a percentage GP4 of $0 \%,<10 \%, 10-25 \%, 26-50 \%$, and $>50 \%$, respectively. Variations between individual gene pathway scores among each \%GP4 category and each GP4 morphology were reflected in the wide range of GPS values and wide range of estimated risk of AP and BCR in all biopsies with GS7. Increasing percentages of GP4 were associated with decreased androgen signaling and cellular organization scores and increased stromal response and proliferation scores. Gene expression pathways 
varied among GP4 morphologies with the cribriform pattern showing the lowest cellular organization and highest proliferation scores and the PFG pattern showing the highest stromal response and lowest androgen signaling scores.

Conclusions: The GPS assay highlights a broad spectrum of tumor aggressiveness in a series of 1143 biopsies containing different GP4 morphologic subtypes and percent GP4. Variation within individual gene expression pathways suggest measurable underlying biologic differences among GS7 prostate cancer may contribute to an increased risk of $\mathrm{AP}$ and $\mathrm{BCR}$.

869 Divergent Molecular Pathogenesis of EBV-Negative Lymphoepithelial Carcinomas Arising in the Nasopharynx and Urinary Bladder Revealed by Next-Generation Sequencing

Benjamin Buelow, Samia Naccache, Guixia Yu, Nancy Joseph, Charles Chiu. University of California, San Francisco, San Francisco, CA.

Background: Lymphoepithelial carcinomas (LELC) are a distinct subgroup of carcinomas rich in admixed lymphoplasmacytic inflammation that can arise in most organs. In some sites such as the nasopharynx, nearly all LELC are driven by EpsteinBarr virus (EBV), whereas LELC of the skin, breast, uterine cervix, and urinary bladder are universally EBV-negative. This study aimed to identify the molecular pathogenesis of EBV-negative LELC arising in the nasopharynx (LELC-N) and urinary bladder (LELC-B) including mutational profile and possible unique viral etiology.

Design: Amongst our pathologic archives, we identified three LELC-B and three LELC-N EBV-negative by immunohistochemistry. Targeted next-generation sequencing (NGS) of 538 cancer genes as well as unbiased sequencing for microbial DNA were performed on this tumor cohort and bioinformatically processed using copy number and mutation algorithms as well as SURPI, a pathogen discovery algorithm.

Results: All three EBV-negative LELC-N cases showed evidence of oncogenic viral infection by NGS: one with EBV (case 1) and two with papilloma virus (HPV; cases 2 and 3). On review, case 2 was noted to arise in the tonsil. Candidate driver mutations overlapped with those previously described in nasopharyngeal carcinoma (NPC, case 1) and oropharyngeal squamous cell carcinoma (SCC, cases 2 and 3). None of the three LELC-B cases showed evidence of viral infection by NGS. Candidate driver mutations overlapped with those previously reported in high grade urothelial carcinoma (HGUC). Table 1: Molecular Features of 6 EBV-Negative Lymphoepithelioma-Like Carcinomas from the Nasopharynx and Bladder

\begin{tabular}{|c|c|c|c|c|}
\hline $\begin{array}{l}\text { Tumor } \\
\text { Type } \\
\end{array}$ & & Candidate Driver Mutations & $\begin{array}{l}\text { Microbial DNA } \\
\text { Detected }\end{array}$ & Molecular Diagnosis \\
\hline \multirow{3}{*}{ LELC-N } & 1 & SMAD3 & EBV & NPC, EBV-positive \\
\hline & 2 & NRAS, MSH6, MLH1, KIT, ATM, ERBB2 & HPV16 & SCC, HPV associated* \\
\hline & 3 & BRCA1, ATRX, TERT promoter & HPV18 & SCC, HPV associated* \\
\hline \multirow{3}{*}{ LELC-B } & 1 & STAG2, TP53, APC & None & HGUC \\
\hline & 2 & TERT promoter, RB1 & None & HGUC \\
\hline & 3 & TP53, PIK3CA^ & None & HGUC \\
\hline
\end{tabular}

Conclusions: These data suggest that EBV-negative LELC of the nasopharynx and urinary bladder are morphologically, but not molecularly, distinct from other high grade carcinomas arising in the nasopharynx and urinary bladder. Each of the LELC-N cases showed evidence of viral infection with either EBV or high-risk HPV, while LELC-B cases lacked detectable viral infection and had a mutation spectrum similar to high grade urothelial carcinoma.

870 Frequent Detection of High Risk HPV and Heterogeneity in Condylomas Associated with Penile Intraepithelial Neoplasia (PeIN). A Laser Capture Microdissection (LCM)-PCR Study of 30 Lesions in 6 Patients

Sofia Canete, Maria Jose Fernandez Nestosa, Nuria Guimera, Diego F Sanchez, Ingrid M Rodriguez, Jose Barreto, David Jenkins, Wim Ouint, Antonio L Cubilla. Instituto de Patología e Investigación, Asunción, Paraguay; Universidad Nacional de Asunción, Asunción, Paraguay; DDL Diagnostic Laboratory, Rijswijk, Netherlands; Facultad de Ciencias Médicas-UNA, Asunción, Paraguay.

Background: Whereas common penile condylomas affecting young patients are related to low risk HPV (LR-HPV), usually 6 or 11, and their histology is benign, little is known about condylomata occurring in association with Penile Intraepithelial Neoplasia.

Design: The aim of this study was to evaluate morphological features and HPV genotypes of 30 penile condylomas identified in 6 patients with penile precancerous lesions. Condylomas were classified as acuminatum and flat, each further subclassified in typical (common condyloma) or atypical. In the former, no atypical cells were identified. In the latter, atypical cells were present in the lower third of epithelial thickness. For HPV detection we used p16, whole tissue section (WTS) and laser capture microdissection (LCM)-PCR.

Results: Patients age ranged from 18 to 74 (average 46 years). PeINs were multicentric with 1-8 lesions per patient. Subtypes were: warty (10 cases), warty-basaloid (4 cases), basaloid ( 2 cases) and differentiated ( 2 cases). Condylomas were also multicentric, ranging from 2 to 6 lesions per patient. P16 was positive in 10 cases (30\%). HPV was detected in 24 of 30 cases $(80 \%)$ as shown in Table 1 . HPV genotypes found are summarized in Table 2 .

\begin{tabular}{|l|l|l|l|}
\hline Condylomas & Number of cases & HR-HPV (\%) & LR-HPV (\%) \\
\hline Typical & 16 & $7(44)$ & $9(56)$ \\
\hline Atypical & 8 & $8(100)$ & 0 \\
\hline
\end{tabular}

\begin{tabular}{|l|l|}
\hline Condyloma Morphology & HPV Genotypes \\
\hline Condyloma acuminata & \\
\hline A. Typical (18 cases) & $11,16,18,44,91,11 / 16$ \\
\hline B. Atypical (3 cases) & 16,39 \\
\hline Flat condylomata & \\
\hline A. Typical (4 cases) & 16,39 \\
\hline B. Atypical (5 cases) & $16,16 / 44 / 66$ \\
\hline
\end{tabular}

There was correspondence of high risk HPV genotypes detected in PeIN and atypical condylomas but not in typical condylomas.

Conclusions: Penile condylomas associated with PeIN are not morphologically homogeneous. In this series, most of the HPV detected in condylomas were of high risk. There was a correlation of type of condyloma and HPV genotype: typical condylomas were more likely to have LR-HPV. HR-HPV was prevalent in flat and atypical condylomas. There was a correspondence of HR-HPV genotypes in PeIN and associated condylomas suggesting a causal link. Some typical condylomas and most atypical condylomas may be precursors of penile carcinoma.

871 Poorly Differentiated Solid (Medullary) Carcinoma: A Distinctive HPV-Related Penile Neoplasm. A Report of 12 Cases

Sofia Canete, Omar OC Clavero, Diego F Sanchez, Arturo Silvero, Francisco Abed, Ingrid M Rodriguez, Laia Alemany, Nubia Munoz, Silvia de Sanjose, Wim Quint, Francesc Xavier Bosch, Antonio L Cubilla. Instituto de Patología e Investigación, Asunción, Paraguay; Institut Català d'Oncologia, Barcelona, Spain; Facultad de Ciencias Médicas-UNA, Asunción, Paraguay; DDL Diagnostic Laboratory, Rijswijk, Netherlands.

Background: A third to half of penile invasive squamous cell carcinomas is HPVrelated. Warty (condylomatous), warty-basaloid and basaloid carcinomas are the most common subtypes associated with HPV. Less frequent HPV-related tumors are clear cell and lymphoepithelioma-like carcinomas. We are reporting a novel penile tumor associated with HPV.

Design: Twelve cases were selected from about 900 penile squamous cell carcinomas, part of an international HPV detection study conducted at the Institut Català d'Oncologia, Barcelona, Spain. Immunostain p16 was performed in all cases. En bloc full tumor staining was the utilized criteria for positivity. For HPV detection, whole tissue section (WTS)-PCR analyses was performed by SPF $_{10}$-DEIA-LiPA $_{25}$ (version 1).

Results: Patients age ranged from 42 to 92 (average 71 years). Tumor was most commonly located in the glans $(70 \%)$. Microscopically a solid medullary-like pattern predominated. Tumors grew in large solid sheets, nests, or had a syncytial pattern. Cells were large and poorly differentiated or anaplastic. Keratinization was minimal or absent. Nuclei were large with prominent nucleoli. Mitoses were numerous. Tumor necrosis was common. There was a moderated to dense inflammatory stromal infiltrate composed of lymphocytes, plasma cells and eosinophils. Deep invasion of corpora cavernosa was frequent. P16 was positive in all cases. HPV was detected in all cases. Genotypes were 66 ( 9 cases), 58, 33, 68 or 73 , one case each.

Conclusions: Medullary carcinomas of the penis are morphologically distinctive HPVrelated high grade neoplasms affecting older individuals. Unlike other HPV-related tumors, where HPV 16 is the prevalent genotype, HPV 66 was detected in $75 \%$ of the cases. More studies are necessary to delineate the epidemiological, clinical and molecular features of this unusual penile neoplasm.

872 Small High Grade Cancer as the Largest Tumor at Radical Prostatectomy (RP)

Trevor Caldwell, Jonathan I Epstein. The Johns Hopkins Medical Institutions, Baltimore, MD

Background: RP where the largest tumor is a small focus of Gleason score $\geq 7$ adenocarcinoma is uncommon.

Design: Of 6613 totally embedded RPs from 2005-2014, 27 (0.4\%) had very small $(<0.2 \mathrm{cc}$. $)$ score $>7$ cancers as the largest cancer in the prostate.

Results: Men ranged from 49 to 68 years old (mean 61.1 years old). RP weighed from 29.1 to $144 \mathrm{~g}$ (mean $65.2 \mathrm{~g}$ ) with PSA values from 0.7 to $14.1 \mathrm{ng} / \mathrm{mL}$ (mean $4.6 \mathrm{ng} / \mathrm{mL}$ ). Family history of prostate cancer was present in 9/24 (37.5\%) patients. Patients were initially biopsied because of rising PSA in 14 (51.8\%), elevated PSA in 10 (37\%), strong family history in $1(3.7 \%)$ and 1 patient was diagnosed on TURP $(3.7 \%)$. Highest grade on $\mathrm{RP}$ was $3+4=7$ in $20(74.1 \%), 4+3=7$ in $4(14.8 \%), 4+5=9$ in $2(7.4 \%)$ and $3+5=8$ in $1(3.7 \%)$. Of 25 biopsies reviewed prior to RP, grades were $3+3=6$ in $10(40 \%), 3+4=7$ in $10(40 \%), 4+3=7$ in $1(4 \%), 4+4=8$ and $4+5=9$ in 2 each $(8 \%)$ with cancer present in 1 to 4 cores (mean 1.6); 1 core positive in $16(64 \%)$ patients. Core tumor volume ranged from $<5 \%$ to $80 \%$ (mean $22.4 \%$ ); $\leq 20 \%$ in a single core in $54 \%$ of cases. At RP, tumors were mostly posterior $(77.8 \%)$ and apical $(59.3 \%)$ while few were at the base $(11.1 \%)$ or anterior $(3.7 \%) .2$ tumors had extra-prostatic extenstion. The side of the tumor on biopsy matched the RP side in $70 \%$ of cases.

Conclusions: The finding of small high grade cancers supports that high grade cancers can arise de novo rather than from de-differentiation from lower grade disease. It can be difficult to identify the high grade component in these cancers on biposy despite their location in areas of the prostate accessible to needle biopsy sampling, where in $40 \%$ of the cases, the biopsy grade was Gleason $3+3=6$. In $30 \%$ of the cases, the tumor on biopsy was not even the small high grade cancer at RP. Despite their small volume, detection was typically due to abnormalities in serum PSA values which were probably unrelated to the tumor, given its small size. Recognition of this phenomenon should be a factor in being cautious with active surveillance for presumed low risk disease. 
873 Distinct Clinicopathological Features in Metanephric Adenoma Harboring BRAF Mutation

Anna Calio', John NEble, Ondrej Hes, Guido Martignoni, Sean R Williamson, Matteo Brunelli, Adeboye O Osunkoya, Lisha Wang, Eva Comperat, Mingsheng Wang, Shaobo Zhang, Kendra Curless, Kristin Post, Hsim-Yee Chang, Lee Ann Baldridge, Gregory MacLennan, Rodolfo Montironi, David J Grignon, Liang Cheng. University of Verona, Verona, Italy; Indiana Univesity, Indianapolis, IN; Charles Univesity Hospital Plzen, Pilsen, Czech Republic; Henry Ford Health System, Detroit, MI; Emory University, Atlanta, GA; Fudan University Shangai Cancer Center, Shangai, China; Groupe Hospitalier Pitie-Salpetriere, Paris, France; Case Western Reserve University, Cleveland, $\mathrm{OH}$; Polytechnic University of the Marche Region, Ancona, Italy.

Background: $B R A F$ mutation has been recently reported in metanephric adenoma. We sought to determine the clinical and morphologic features of $B R A F$ mutated metanephric adenoma and correlate $B R A F$ mutation with BRAF V600E immunohistochemical staining results.

Design: A series of 45 metanephric adenomas were analyzed for the occurrence of $B R A F$ mutation (BRAF V600E, BRAF V600D, BRAF V600K and BRAF V600R) using the BRAF RGQ PCR kit (Qiagen). Immunohistochemistry was performed using monoclonal mouse antibody VE1 (Spring Bioscience), recognizing the BRAF V600E mutant protein. Results: Thirty-eight of 45 cases $(84 \%)$ showed $B R A F$ V600E mutation; none of the other $B R A F$ variants were detected. Of $38 B R A F$ mutated neoplasms, 34 cases showed positive VE1 immunostainings (sensitivity $89 \%$, specificity $100 \%$ ). The following features were associated with $B R A F$ V600E mutation: older patients $(\mathrm{p}=0.001)$, female predominance $(\mathrm{p}=0.005)$, the presence of a predominant acinar component $(\mathrm{p}=0.02)$, and the presence of edematous stroma $(\mathrm{p}=0.05)$.

Conclusions: $B R A F$ mutated metanephric adenomas were associated with older age, female predominance, and the presence of a predominant acinar component and edematous stroma. A subset of $B R A F$ mutated metanephric adenomas was not detected by VE1 immunostaining.

\section{Mixed Epithelial and Stromal Tumor of Kidney}

Anna Calio', John N Eble, David J Grignon, Brett Delahunt. University of Verona, Verona, Italy; Indiana University, Indianapolis, IN; Wellington Medical School, Wellington, New Zealand.

Background: Mixed epithelial and stromal tumor of kidney is a controversial neoplasm. Published studies have been small and have left gaps in our understanding of its morphology.

Design: We studied hematoxylin and eosin-stained sections (from 1 to 32 blocks per tumor, median $=6$ ) from 60 tumors $(51 \mathrm{~F}$, median ages: $50 \mathrm{~F} 68 \mathrm{M})$, collecting data on stromal, epithelial, and architectural elements.

Results: Stroma: smooth muscle in 41 tumors ( $5 \%$ - 90\%of the stromal compartment), collagenized fibrous in $42(5 \%-90 \%)$, hypercellular spindle cell in $27(5 \%-90 \%)$, edematous fibrous in $19(5 \%-40 \%)$, fat in $17(<1 \%-20 \%)$, calcification in 16 , clusters of thick-walled vessels in 13, emosiderin-bearing macrophages in 12 , reminiscent of ovarian in 10. Combinations of these diverse stromal elements were common: 12 tumors contained 2, 30 contained 3 or 4,14 contained 5 or 6 , and 4 contained 6 or 7 . Epithelium: tiny crowded glands in 29 tumors (5\%-80\%), branching glands in 27 $(<5 \%-70 \%)$, round glands lined by columnar epithelium in $23(5 \%-80 \%)$, reminiscent of thyroid follicles in $18(<5 \%-85 \%)$, reminiscent of phyllodes tumor and spatulate papillae in $17(5 \%-90 \%)$, urothelium in $17(5 \%-30 \%)$, reminiscent of nephrogenic adenoma in 12 (1\%-35\%), complex papillae in 8 , ciliated in 6 , subnuclear vacuoles in 2 , mucinous goblet cells in 1 , squamous in 1 . Combinations of these elements were common: 16 tumors contained 2, 7 contained 3, 5 contained 4, 7 contained 5, 5 contained 6 , and 6 contained $>7$. Architecture: A solid architecture with only a few small cysts was found in $31 \%$ of the tumors. Solid and cystic architecture was found in $39 \%$ and predominantly cystic architecture in $30 \%$.

Conclusions: In the stroma, components of fat and smooth muscle were found much more frequently than in previous reports. In the epithelial compartment, glands reminiscent of nephrogenic adenoma were previously reported in only one series. Branching glands and ducts in nearly $50 \%$ of tumors, urothelium in $27 \%$, and ciliated epithelium in $10 \%$ of tumors are much more frequent than previously reported. Epithelial differentiation to include squamous and mucous goblet cells has not been well recognized. Phyllodes-like and spatulate papillary architecture have seldom been reported. The strong tendency for mixed epithelial and stromal tumors to contain multiple types of stroma and epithelium has not previously been quantitated. The strong predominance of solid and solid and cystic architecture in mixed epithelial and stromal tumors is an important feature of the entity.

\section{Cystic Nephroma in Adults}

Anna Calio', John N Eble, David J Grignon, Brett Delahunt. University of Verona, Verona, Italy; Indiana University, Indianapolis, IN; Wellington Medical School, Wellington, New Zealand.

Background: Cystic nephroma is a controversial neoplasm. Published studies have been small and have left gaps in our understanding of its morphology and evolution. Design: We studied hematoxylin and eosin-stained sections (from 1 to 39 blocks per tumor, median $=6$ ) from 46 tumors from adults $(41 \mathrm{~F}$, median ages: $52 \mathrm{~F} 44 \mathrm{M})$, collecting data on stromal, epithelial, and architectural elements.

Results: Stroma: hypocellular fibrous stroma was $>80 \%$ of the stromal compartment in 11 tumors (median age 61 ) and $<10 \%$ in 15 (median age 48 ) (age difference: $\mathrm{p}=0.0001$ ). Cellular stroma $>40 \%$ was present in 15 (median age 49 ) tumors while $<30 \%$ was present in 18 (median age 58 ) (age difference: $\mathrm{p}=0.02$ ). Hypocellular stroma predominated in older patients with smaller tumors while hypercellular stroma predominated in younger patients with larger tumors. No smooth muscle appeared within the septa. Irregular calcifications were present in $22(48 \%)$ and clusters of macrophages in 15 tumors (33\%). Epithelium: In addition to flattened and hobnail epithelium, foci of clear cells with or without apical eosinophilic granules were seen 10 tumors $(22 \%)$. Small, rare, foci with multiple layers of epithelium were seen in 5 (11\%). Architecture: A pseudocapsule containing smooth muscle was present around 12 tumors $(26 \%)$. The tumors were composed entirely of cysts with septa of variable thickness. Small foci of stroma of moderate cellularity in which a spectrum of sizes of epithelial elements ranging from a handful of cells with no lumen, to tiny cysts with pinpoint lumens to slightly larger cysts were found in 14 tumors (54\%). Entrapped renal tubules were present in septa in 5 tumors $(11 \%)$. In some of the smaller tumors, a pseudocapsule was absent and cystic nephroma stroma infiltrated the interstitium between renal tubules.

Conclusions: The findings support the concept of cystic nephroma originating as a stromal proliferation in the renal interstitium (probably the medulla) with the epithelial component arising from a stromal-epithelial transition which can be ongoing even in larger tumors with substantial amounts of hypocellular fibrous stroma in the septa. The findings also support the concept of the stroma maturing with decreasing cellularity, ultimately becoming hypocellular and collagenized.

\section{GATA3 Is a Sensitive Marker for Extramammary Paget's Diseases} of Male Genital Region

Dengfeng Cao, Yunquan Guo, Yan Song, Lixin Zhou, Junqiu Yue, Xun Zhang, Ming Zhao, Li Sun, Zhongwu Li. Washington University School of Medicine, Saint Louis, MO; Xinjiang Cancer Hospital, Urumqi, China; Chinese Academy of Medical Sciences Cancer Hospital, Beijing, China; Peking University Cancer Hospital, Beijing, China; Hubei Cancer Hospital, Wuhan, China; Zhejiang People's Hospital, Hangzhou, China. Background: GATA-binding protein 3 (GATA3) is a zinc-finger transcription factor involved in cell development and differentiation. Recent studies have shown that GATA3 is a useful diagnostic marker for breast and urothelial carcinomas. Extramammary Paget's disease (PD) in male genital region is uncommon but it can evolve into an invasive adenocarcinoma and rarely metastasize. Morphologically the invasive and metastatic components of PDs may mimic urothelial carcinoma especially in the metastatic setting, posing diagnostic challenges. In this study using immunohistochemical staining we investigated GATA3 expression in a series of PDs in male genital region to assess its diagnostic utility in these tumors. We also compared GATA3 to gross cystic disease fluid protein 15 (GCDFP15) in these tumors.

Design: Thirty-seven PDs from male genital region (penis 3, perineum 1, 33 scrotum) were included: 16 of them also with an invasive adenocarcinoma component and 12 also with metastasis. One to two representative tissue blocks from each case were retrieved to generate $4 \mathrm{um}$ unstained slides for immunohistochemical staining with antibodies to GATA3 and GCDFP15. The staining was semi-quantitatively scored as negative (no tumor cells stained), $1+(1-25 \%), 2+(26-50 \%), 3+(51-75 \%)$, and $4+$ (76-100\%). Only nuclear staining was considered positive for GATA3. For GCDFP15, the staining was cytoplasmic.

Results: The in-situ component of PD showed positive GATA3 staining in all 37 cases, including $1+$ in $4,2+$ in $2,3+$ in 2 , and $4+$ in 29 . The associated invasive component showed positive GATA3 staining in $15 / 16$ cases, including $1+$ in $2,2+$ in $2,3+$ in 1 , and $4+$ in 10. Positive GATA3 staining was seen in the metastatic component of PD in $11 / 12$ cases, including $1+$ in $1,2+$ in $1,3+$ in 1 , and $4+$ in 8 . Positive GCDFP 15 staining was seen in the in-situ component of PD in $18 / 37$ cases $(1+$ in $12,2+$ in $4,3+$ in 0 , $4+$ in 2$)$, in the invasive component in $11 / 16$ cases $(1+$ in $6,2+$ in $1,3+$ in $0,4+$ in 4$)$, and in the metastatic component in $10 / 12$ cases $(1+$ in $3,2+$ in $2,3+$ in 1 , and $4+$ in 4$)$. Conclusions: Conclusion: GATA3 is a highly sensitive marker for PD in male genital region. The high sensitivity is generally maintained in the invasive component and metastatic component for these tumors. GATA3 is relatively more sensitive than GCDFP15 for PD in male genital region.

\section{Unraveling the Molecular Characteristics of Urachal} Adenocarcinoma Starting from an Extreme-Responder Patient

Mireia Castillo-Martin, Ana Collazo Lorduy, John P Sfakianos, Carlos Cordon-Cardo, Matthew Galsky. Icahn School of Medicine at Mount Sinai, New York, NY.

Background: Urachal carcinoma is a rare bladder malignancy, which usually presents at an advanced stage and harbors poor prognosis. Starting from an extreme-responder patient, the objective of this study was to improve the molecular understanding of this uncommon disease to guide standard of care.

Design: A 34-year-old male was diagnosed in August 2013 with bladder urachal adenocarcinoma with lung metastasis. A partial cystectomy was performed to control hematuria and he received two lines of chemotherapy: Gemcitabine-FLP (5-FU, leucovorin, cisplatin) with stabilization of disease and Carboplatin-Paclitaxel with progression of disease. Targeted exome sequencing was performed in the primary tumor (Foundation Medicine) in an attempt to identify potentially "actionable" genomic alterations. Furthermore, formalin-fixed paraffin-embedded tissue specimens from other 10 patients with urachal adenocarcinoma and clinical follow-up data were retrospectively identified in our institution's archives after chart review (2000-2014). DNA extraction for targeted exome sequencing, FISH and immunohistochemical analyses were performed in all cases.

Results: Targeted exome sequencing of the bladder tumor from the first patient revealed amplification of EGFR and E-cadherin amongst other molecular alterations. Amplification of EGFR was confirmed by FISH. Thus, Cetuximab, an EGFR inhibitor, was administered based on two considerations: 1) anecdotal reports of patients with urachal cancer responding to "colon cancer-type" regimens including chemotherapy plus EGFR inhibition, 2) the previously reported mechanistic role of E-cadherin in modulating sensitivity to EGFR inhibition in vitro. The patient achieved a partial response to single-agent Cetuximab lasting longer than 10 months, and is still alive after more than two years. Sequencing results from the other 10 cases are currently being 
analyzed to determine the frequency of EGFR amplification, E-cadherin amplification, and status of other potentially actionable targets. FISH of EGFR revealed amplification/ Chromosome 7 polysomy in 6 of the 10 cases, exposing the importance of this pathway in urachal adenocarcinoma.

Conclusions: Targeted exome sequencing may identify "actionable" targets in patients with urachal cancer, a disease with no standard therapeutic approaches, opening novel treatment opportunities to improve patient outcome.

878 Very-Small-Nuclear Circulating Tumor Cell (vsnCTC) as a Putative Biomarker for Visceral Metastasis (VM) in Metastatic Castration-Resistant Prostate Cancer (mCRPC)

Jie-Fu Chen, Hao Ho, Elisabeth Hodara, Ann Go, Alexander Ureno, Elizabeth Kaufman, Margarit Sievert, Daniel Luthringer, Zunfu Ke, Leland WK Chung, Ker-Chau Li, Hsian-Rong Tseng, Edwin M Posadas. Cedars-Sinai Medical Center, Los Angeles, CA; University of California, Los Angeles, Los Angeles, CA.

Background: Patients with metastatic castration-resistant prostate cancer (mCRPC) who develop visceral metastasis (VM) have a poorer clinical outcome in comparison to those without VM. Their clinical course is aggressive and culminates in organ failure as this process is often discovered late in the disease course. There are no existing tests that identify men at risk for VM other than radiography. Our team has identified an association between the presence of very-small-nuclear circulating tumor cells (vsnCTCs) and VM. We hypothesized that vsnCTC that can predict the development of VM and monitor the response to anti-cancer treatment.

Design: In our database we identified mCRPC patients who had progressed through next generation hormonal maneuvers such as abiraterone, enzalutamide, or an equivalent drug. Serial blood specimens were used for vsnCTC enumeration using NanoVelcro CTC Assay as previously published (Chen JF et al. Cancer. 2015). The vsnCTC counts were related to the presence and development of VM (evaluated by radiography) as well as the response to anti-cancer treatment.

Results: Blood specimens were identified from 28 patients who met the eligibility criteria; $16 / 28$ patients presented with VM and 12/28 had bone-only disease at their first CTC enumeration. Five out of 12 non-VM patients developed VM during follow-up, and vsnCTCs were detected 86-196 days prior to radiographic detection of VM (true positive); 3/12 had vsnCTCs detected but no VM was found by the time of analysis (false positive). None of the vsnCTC(-) patients developed VM. vsnCTCs were detected in $21 / 21 \mathrm{VM}$ patients compared to $3 / 12$ non-VM patients. Reduction of vsnCTC count occurred at initiation of anti-cancer treatment; transition from vsnCTC $(-)$ to $\operatorname{vsnCTC}(+)$ was seen prior to progression under the treatment.

Conclusions: vsnCTC is associated with the presence of VM and is a potential biomarker for predicting the development of VM and monitoring the treatment response in mCRPC. Transition from vsnCTC( -$)$ to vsnCTC $(+)$ was associated with the development of VM and progression under the treatment.

879 CDK2 Inhibitory Phosphorylation Regulates Spermatogenesis and Stem Cell Maintenance

Xueyan Chen, Hui Zhao, Bruce Clurman, James Roberts, Keith Loeb. University of Washington, Seattle, WA; Fred Hutchinson Cancer Research Center, Seattle, WA

Background: Inhibitory phosphorylation of cyclin-dependent kinase 2 (CDK2) has been shown to play a major role in controlling cyclin E-associated kinase activity in G1-S transition. The mice in which the two Cdk2 inhibitory phosphorylation sites were mutated to nonphosphorylatable residues ((T14A and Y15F), showed a similar life span as wild-type (WT) littermates. However, the CDK2AF allele had a dominant effect on male fertility; CDK2AF heterozygous males (CDK2+/AF) were infertile. We propose CDK2 inhibitory phosphorylation is essential for spermatogenesis and may also regulate spermatogonial stem cell maintenance.

Design: We examined the histology of testes of WT and CDK2+/AF mice at different ages. We then explored the potential mechanism of defect in spermatogenesis in CDK2+/ AF mice. Furthermore, we studied the impact of CDK2+/AF on hematopoietic stem cell maintenance.

Results: CDK2+/AF mice showed completely depleted spermatogonial stem cells and maturing spermatocytes in the testis at 10 weeks of age and no sperms were detected in the epididymis resembling Sertoli cell-only syndrome. No obvious difference was seen between WT and CDK2+/AF testes at 9 days of age; however, at day 12, defects became apparent with fewer cells present in the seminiferous tubules of CDK2+/AF than the WT testes. By day 15, most of the spermatocytes had disappeared in the CDK2+/ $\mathrm{AF}$ mice and no spermatid were found at day 18, when a transition from meiosis I to meiosis II occurs, suggesting an arrest of meiotic progression at the pachytene stage of spermatogenesis. Furthermore, CDK2+/AF spermatogonial stem cells had decreased proliferative rate and underwent apoptosis by day 20 resulting in a complete loss of stem cells needed for additional waves of spermatogenesis. There was also a delay in recruitment of meiotic recombination protein (Dmc1) and repair of DNA double-strand breaks (DSB) detected by $\gamma-\mathrm{H} 2 \mathrm{ax}$ in the $\mathrm{CDk} 2+/ \mathrm{AF}$ mice. These studies indicate that the inhibitory phosphorylation of CDK2 is required for both male germ cell meiosis and spermatogonial stem cell maintenance. Additional studies showed CDK2+/AF have a partial defect in stress erythropoiesis, suggesting a regulatory role in hematopoietic stem cell maintenance.

Conclusions: Our data demonstrate that inhibitory phosphorylation of CDK2 is essential for spermatogenesis and germ cell maintenance in various stages. It also plays an important role in stress erythropoiesis.
880 Expression of Heterochromatin Protein 1 in Renal Cortical Tumors and Identification of Its Target Genes

Lin Cheng, Tracy K Hale, Maria S Tretiakova. University of Washington, Seattle, WA; Massey University, Palmerston North 4410, New Zealand.

Background: Heterochromatin Protein 1 (HP1) isoforms $\alpha$ and $\beta$ are essential in chromatin packaging and regulate gene expression by decreasing the chromatin plasticity. Reduction of HP1 $\alpha$ expression has been reported in thyroid carcinomas and medulloblastomas, whereas decrease of HP1 $\beta$ expression has been observed in colon cancers, melanomas and prostate cancers. Here we assessed the expression of HP $1 \alpha$ and HP $1 \beta$ in various types of renal cortical tumors, analyzed effect of HP1 $\alpha$ knockdown, and identified its target genes in a cancer cell line.

Design: Immunohistochemical (IHC) studies of HP $1 \alpha$ and HP1 $\beta$ were performed on tissue microarrays (TMA) including 104 clear cell renal cell carcinomas (CRCC), 66 papillary renal cell carcinomas (PRCC), 30 chromophobe renal cell carcinomas (CHRCC), 12 oncocytomas (ONC), and 9 normal kidneys (NK). The nuclear staining intensity was graded as negative $(0)$, weak $(1+)$, moderate $(2+)$, or intense $(3+)$. HP $1 \alpha$ was knocked down in breast cancer cell line MCF7. The heterochromatin formation and cell morphology were studied by immunofluorescence. RNAseq was used to identify the target genes of HP1 $\alpha$.

Results: Compared to normal kidneys, CRCCs, PRCCs and CHRCCs exhibit significantly decreased expression of both HP1 isoforms $(\mathfrak{p}<0.0001)$. ONCs retain normal level of HP $1 \alpha$, and $<1 / 2$ of HP $1 \beta$. The Fuhrman grades of CRCCs and PRCCs are not correlated with the expression level of either isoform $\left(r^{2}=0.0001-0.06\right)$. The HP1 $\alpha$ knockout cell line showed compromised heterochromatin, altered cell adhesions, and increased ability to invade. Furthermore, 130 genes were identified as the targets of HP1a, which had altered expression in knockdown cell line.

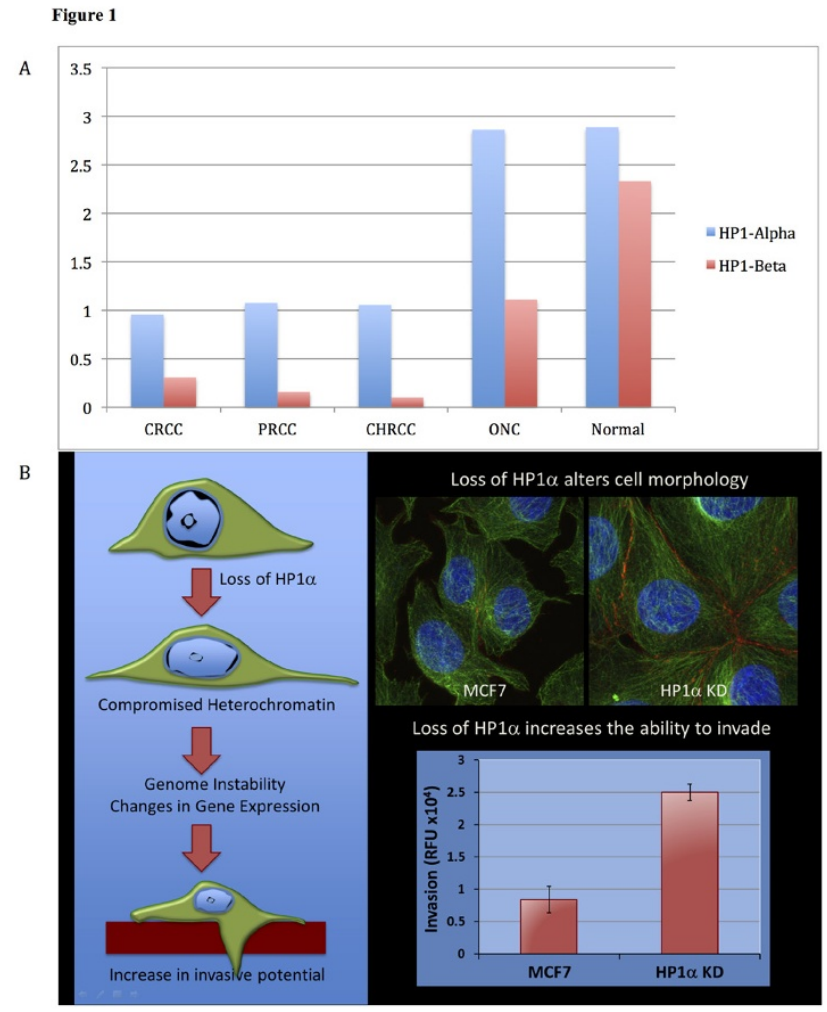

Conclusions: Reduced expression of both HP1 $\alpha$ and HP1 $\beta$ are present in all 3 types of renal cell carcinoma studied, and are not affected by the nuclear grade of CRCCs and PRCCs. Oncocytoma displays near-normal HP1 $\alpha$ and significantly decreased HP1 $\beta$ expression, which is a potential diagnostic marker for differentiating ONCs from CHRCCs. Loss of HP1 $\alpha$ in cancer cell line leads to altered cell morphology, increased invasive ability and global gene expression changes including 130 genes involved in epithelial-to-mesenchymal transformation.

881 Prostate Intraductal Carcinoma, Multifocality and Bilateral Significant Disease in Radical Prostatectomy Specimens from United States and Japan

Yu-Ching Peng, Toyonori Tsuzuki, Max Kong, Jianhong Li, Fang-Ming Deng, Jonathan Melamed, Ming Zhou. New York University, New York, NY; Japanese Red Cross Nagoya Daini Hospital, Nagoya, Japan.

Background: Prostatecancers (PCa) in North America and Japan differ in their biological and epidemiological features. One previous study showed that Japanese PCa is more likely to have larger, higher grade and stage tumors compared with US PCa. However, the incidence of intraductal carcinoma (IDC), multifocality and bilateral significant disease have not been studied.

Design: We reviewed233 radical prostatectomies (111 US; 122 Japan) and generated maps documenting individual tumor foci, dominant nodules, Gleason score (GS), 
IDC, extraprostatic extension (EPE), seminal vesicle invasion (SVI), pathological stage and IDC. Clinically significant disease is defined by $\geq 5 \mathrm{~mm}$ in size, or GS $\geq 7$, or presence of EPE or SVI.

Results: US PCa had a higher pathological stage ( $55 \%$ pT2 and $45 \%$ pT3 in US vs $71 \%$ pT2 and $29 \%$ pT3 in Japan, p $<0.01$ ). Japanese PCa had a higher Gleason score $(19.8 \% \mathrm{GS} 6,61.3 \% \mathrm{GS} 7,18.9 \% \mathrm{GS}>8$ in US vs $9.8 \% \mathrm{GS} 6,77.1 \% \mathrm{GS} 7,13.1 \% \mathrm{GS}$ $>8$ in Japan, $\mathrm{p}<0.05$ ). The number of tumor foci was significantly higher in Japanese $\mathrm{PCa}$ (3.8 Japan vs $2.9 \mathrm{US}, \mathrm{p}<0.05$ ). The incidence of bilateral significant disease was not significantly different between Japanese and US PCa (68.6\% Japan vs $57 \%$ US, $\mathrm{p}>0.05$ ). The incidence of IDC was significantly higher in Japanese PCa (35.3\% Japan vs $12.6 \%$ US, $\mathrm{p}<0.01$ ). The higher incidence of IDC in Japanese PCa is independent of tumor stage (Table 1) and Gleason score (Table 2).

Table 1. Intraductal carcinoma in the US and Japanese patients stratified by tumor stage

\begin{tabular}{|l|l|l|l|l|l|}
\hline Stage & \multicolumn{2}{|l|}{$\mathrm{pT} 2$} & $\mathrm{p}$ p 3 & \\
\hline & IDC+ & IDC- & IDC+ & IDC- & total \\
\hline US & $4(6.6 \%)$ & $57(93.4 \%)$ & $10(20 \%)$ & $40(80 \%)$ & 111 \\
\hline Japan & $21(24.1 \%)$ & $66(73.9 \%)$ & $22(62.9 \%)$ & $13(37.1 \%)$ & 122 \\
\hline p value & $<0.01$ & $<0.01$ & & \\
\hline
\end{tabular}

Table 2. Intraductal carcinoma in the US and Japanese patients stratified by Gleason score (GS) [table2]

\begin{tabular}{|l|l|l|l|l|l|l|l|}
\hline GS & \multicolumn{2}{|l|}{6} & \multicolumn{1}{l|}{7} & \multicolumn{2}{l|}{8 and above } & \\
\hline & IDC + & IDC- & IDC + & IDC- & IDC + & IDC- & total \\
\hline US & $0(0 \%)$ & 22 & $8(11.8 \%)$ & 60 & $6(28.6 \%)$ & 15 & 111 \\
\hline Japan & $0(0 \%)$ & 12 & $29(30.9 \%)$ & 65 & $14(87.5 \%)$ & 2 & 122 \\
\hline p value & $>0.05$ & $<0.01$ & $<0.01$ & & \\
\hline
\end{tabular}

Conclusions: Japanese PCa has higher GS, number of PCa foci per prostate gland and lower pathological stage. We also found higher incidence of IDC in Japanese $\mathrm{PCa}$ independent of the pathological stage and Gleason score. It may reflect distinct biological behaviors of PCa in Japanese patients.

882 Breaking down the Contemporary Gleason Pattern (GP) 4 in Prostate Cancers: Should We Report the Percentage of Pattern 4 in Radical Prostatectomies (RPs)?

Bonnie Choy, Blake Anderson, Gladell P Paner. The University of Chicago, Chicago, IL. Background: The 2014 ISUP grade groupings placed emphasis on the impact of the increasing amount of GPs 4 and 5 in predicting the behavior of prostate cancer. This study was conducted to examine the influence of the amount of GP 4 and its different architectural components in the behavior of Gleason score (GS) 7 cancers in RP.

Design: 585 RPs with GS 6 and $7(3+4$ and 4+3) prostate cancers from the surgical pathology files (2003 to 2006) of a tertiary institution were reviewed. The RPs were evaluated by 2 of the authors for re-grading according to the 2014 ISUP modified Gleason grading criteria, percentages of GP 4 and its architectures (e.g., glomeruloid, ill-formed, cribriform, fused glands) present. PSA biochemical recurrence (BCR) was used as the end point. Median follow-up was 75 months.

Results: Prostate cancer GS was $3+3(40.2 \%), 3+4(49 \%)$, and $4+3(10.8 \%)$. In GS 7, GP 4 was $<5 \%$ (18.6\%), $5-10 \%(23.4 \%), 11-20 \%(16 \%), 21-30 \%(13.4 \%), 31-40 \%$ (6.3\%), $41-50 \%$ ( $4.3 \%), 51-60 \%$ (7.7\%), 61-70\% (6.6\%), $71-80 \%$ (3.1\%), and $>80 \%$ $(0.6 \%)$. In GS 7, GP 4 exhibited cribriform (43.1\%), ill-formed (39.7\%), fused (38\%) and glomeruloid (20.9\%) glands; admixture were common. BCR was seen in $13.7 \%$ of all cases. Significantly different rates of BCR were observed among GS $3+3(2.1 \%)$, GS $3+4(17.4 \%)$, and GS $4+3(39.6 \%)(p<0.001)$. BCR of GP 4 by quartile is in table 1. When percent of GP 4 was further divided, BCR was $0 \%(2.1 \%),<5 \%(10.7 \%)$, $5-10 \%(15.8 \%), 11-20 \%(16 \%), 21-30 \%(25.5 \%), 31-40 \%(18.1 \%), 41-50 \%(33.3 \%)$, $51-60 \%(33.3 \%), 61-70 \%(34.7 \%), 71-80 \%(63.6 \%)$, and $>80 \%(100 \%)(\mathrm{p}<0.001)$. BCR by GP4 architectures is in table 2 .

\begin{tabular}{|l|l|}
\hline \% of GP 4 & BCR \\
\hline $1-25$ & $14.2 \%$ \\
\hline $26-50$ & $25 \%$ \\
\hline $51-75$ & $32 \%$ \\
\hline$>75$ & $69.2 \%$ \\
\hline
\end{tabular}

\begin{tabular}{|l|l|l|l|}
\hline GP 4 Architecture & $\begin{array}{l}\text { BCR With } \\
\text { Architecture }\end{array}$ & $\begin{array}{l}\text { BCR Without } \\
\text { Architecture }\end{array}$ & p \\
\hline Cribriform $(\mathrm{n}=151,43.1 \%)$ & $30.4 \%$ & $7.8 \%$ & $<0.001$ \\
\hline Ill-formed $(\mathrm{n}=139,39.7 \%)$ & $22.3 \%$ & $10.9 \%$ & 0.001 \\
\hline Fused $(\mathrm{n}=133,38 \%)$ & $19.5 \%$ & $11.9 \%$ & 0.025 \\
\hline Glomeruloid (n=73, 20.9\%) & $10.9 \%$ & $14 \%$ & 0.47 \\
\hline
\end{tabular}

Conclusions: This study showed that cribriform, ill-formed, and fused glands architectures individually have impact on BCR, with cribriform glands having the strongest correlation. Increasing percentages of GP 4, by quartiles and in select deciles, correlates with BCR. This study suggests the importance of reporting percentage of GP 4 in RPs, at least by quartile. Larger number of cases are needed to determine the impact on BCR of further breaking down the GP4 quartiles.
883 Programmed Death Ligand-1 (PD-L1) Status in North American Cohort of Penile Squamous Cell Carcinoma

Margaret Cocks, Diana Taheri, Mark W Ball, Stephania M Bezerra, Maria DC Rodriguez, Alan Meeker, Trinity J Bivalacqua, Alcides Chaux, Arthur Burnett, George J Netto. Johns Hopkins Medical Institution, Baltimore, MD; Isfahn University of Medical Sciences, Isfahan Kidney Diseases Research Center, Isfahan, Islamic Republic of Iran. Background: Penile squamous cell carcinoma (SCC) is primarily treated with surgical resection. Locally advanced and metastatic disease require a multidisciplinary approach. However, mortality and morbidity rates remain high in such patients where a molecular target of therapy is currently sought. Programmed Death Ligand-1 (PD-L1) is a co-inhibitory molecule that impairs the T-cell response by downregulating T-cell proliferation and cytokine production. Tumors cells often upregulate PD-L1 and thereby evade the host immune system. PD-L1 has been successfully targeted in solid tumors. We sought to determine the expression of PD-L1 in penile cancers.

Design: Fifty-three invasive penile SCCs diagnosed between 1985 and 2013 were retrieved from our surgical pathology archives. Representative formalin-fixed, paraffinembedded archival blocks were used for the construction of 2 high-density tissue microarrays (TMAs). PD-L1 was investigated by immunohistochemistry using rabbit monoclonal anti PD-L1 antibody (Cell Signaling, E1L3N, 1:100). PD-L1 positivity was defined as presence of any extent (percent) of tumor cell membranous positivity in one or more representative TMA spot. Findings were correlated with clinicopathologic parameters and outcomes by univariate and multivariate analyses.

Results: Overall 20/53 (38\%) of penile SCCs had positive PD-L1 expression. PD-L1 was expressed by a significant proportion of advanced penile SCC. $41 \%(13 / 32)$ of stage pT2 or more SCC and 38\% (6/16) of tumors with lymph node metastasis were positive for PD-L1.

On univariate analysis, PD-L1 expression did not correlate with patient age, tumor location, histologic subtype, tumor stage, anatomic depth of invasion or tumor grade. PD-L1 positivity did not predict tumor recurrence, progression or cancer related death. On multivariate analysis, only $\mathrm{p} T$ stage $(\mathrm{p}=0.01)$, presence of lymphovascular invasion $(p=0.03)$, perineural invasion $(0.001)$ and presence of urethral invasion $(p=0.008)$ were significantly associated with cancer related death.

Conclusions: Our current study is the largest to assess expression of PD-L1 in clinically annotated North American cohort of penile SCC. PD-L1 was expressed by $38 \%$ of penile SCC including those with advanced disease. Our findings support a rational for targeting immune-checkpoint inhibitors pathway in advanced penile SCC. PD-L1 status was not predictive of tumor recurrence, progression or cancer related death.

884 Regional and Racial Disparities in Bladder Cancer Pathology Undergoing Secondary Review at a Tertiary Referral Center in Alabama Robin Collingwood, Win Shun Lai, Soroush Rais-Bahrami, Jennifer B Gordetsky. University of Alabama at Birmingham, Birmingham, AL.

Background: For bladder cancer, depth of invasion is vital to determine whether a cystectomy is necessary. Regional differences in health care providers and resources can play a significant role in a workup and diagnosis. The purpose of this study was to review bladder specimens referred to our facility to determine what demographic trends were associated with changes in pathologic diagnoses.

Design: A retrospective review of 165 bladder specimens sent to our pathology department for 2nd opinion was performed; any changes in the pathologic grade, stage, or tumor type were noted. Demographic data for each patient and the county they resided in was obtained through medical records and the US Census. Statistical analysis was performed to determine any significant relationship between a change in pathology and patient demographics.

Results: $35 / 165(21 \%)$ patients had a change in diagnosis, of which 20/35 (57\%) had a change in stage, 10/35 (29\%) tumor type, 6/35 (17\%) grade, and 8/35 (23\%) presence of muscularis propria. $2 / 35(6 \%)$ patients had a malignant diagnosis changed to benign. $8 / 35(23 \%)$ patients were downstaged from $\mathrm{T} 2$ to $\mathrm{T} 1$. Changes in various pathologic diagnoses were statistically associated with counties with a higher percentage of Caucasians compared to African Americans, a higher percentage of uninsured citizens, and increased distance from our tertiary referral center. Caucasian patients were more likely to live in counties with a higher average population income and lower percentage of citizens with health insurance. Areas with a higher percentage of African Americans was significantly associated with increased college graduates, decreased uninsured, but increased citizens below poverty level. 


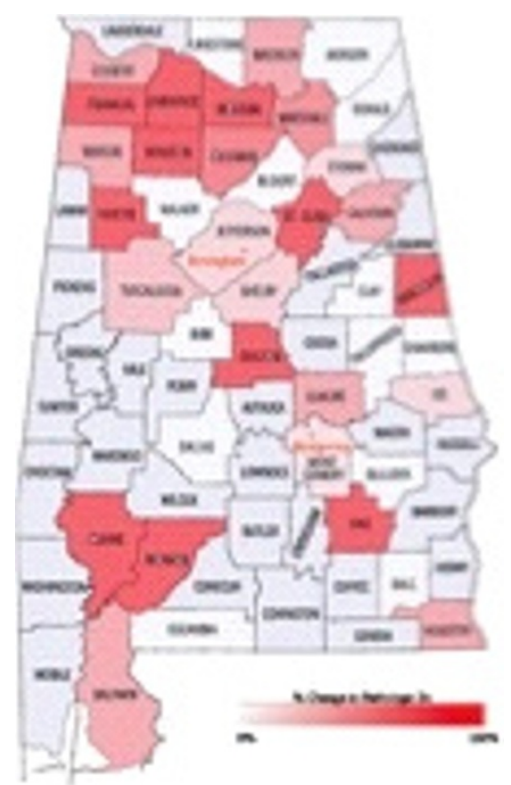

Conclusions: A significant number of patients with bladder cancer had a change in diagnosis upon secondary review at a tertiary referral center. Disparities in pathologic diagnoses were associated with an increased distance from our tertiary referral center and observed from areas with a higher percentage of Caucasians and uninsured citizens.

885 Mutational Profile Using Next Generation Sequencing May Aid in Distinguishing Urachal Adenocarcinoma from Bladder Adenocarcinoma Kristine M Cornejo, Gladell P Paner, Keith Tomaszewicz, Xiuling Meng, Vikas Mehta, Sahussapont J Sirintrapun, Guliz Barkan, Ediz F Cosar, Lloyd Hutchinson. University of Massachusetts Medical School and UMass Memorial Healthcare, Worcester, MA; University of Chicago, Chicago, IL; Loyola University Medical Center, Maywood, IL; Memorial Sloan Kettering Cancer Center, New York, NY.

Background: Urachal adenocarcinoma (UAC) has striking morphologic and immunohistochemical overlap with colorectal adenocarcinoma (CAC) and bladder adenocarcinoma (BAC). Activating mutations in $K R A S$ and $B R A F$ are frequently found in sporadic CAC. To date, the mutational status in UAC and BAC has not been well investigated. The aim of the study was to determine whether the molecular profile of these neoplasms may aid in the diagnosis.

Design: Sixteen UACs ( 9 mucinous, 4 intestinal, 1 signet ring cell, 2 not otherwise specified [NOS] types), and 4 BACs (3 intestinal, 1 signet ring cell) were included in the study. Next generation sequencing (NGS) analysis of 50 cancer "hotspot" gene mutations using the Ampliseq Cancer Hotspot Panel v2 was performed.

Results: RAS mutations were identified in 9 of 16 (56\%) UACs (8 KRAS; 1 NRAS) and none of the $\mathrm{BACs}(0 \%$ ) (table 1), of which $50 \%$ are rare non codon $12 / 61$ mutations. TP53 mutations were found in both UACs $(7 / 16 ; 44 \%)$ and BACs $(4 / 4 ; 100 \%)$. GNAS $(\mathrm{n}=4)$, SMAD4 $(\mathrm{n}=3)$ and BRAF $(\mathrm{n}=1)$ mutations were only found in UACs. In contrast, $\mathrm{APC}(\mathrm{n}=2)$ mutations were only found in BACs.

\begin{tabular}{|c|c|c|c|c|c|c|}
\hline Case & RAS & BRAF & TP53 & GNAS & SMAD4 & APC \\
\hline UAC1 & p.A146T & p.G469A & p.R273C & p.R201H & $\mathrm{N}$ & $\mathrm{N}$ \\
\hline UAC2 & p.A146T & $\mathrm{N}$ & $\mathrm{N}$ & $\mathrm{N}$ & $\mathrm{N}$ & $\mathrm{N}$ \\
\hline UAC3 & p.A146T & $\mathrm{N}$ & $\mathrm{N}$ & p.R201H & $\mathrm{N}$ & $\mathrm{N}$ \\
\hline UAC4 & p.A59E & $\mathrm{N}$ & p.R196Q & $\mathrm{N}$ & $\mathrm{N}$ & $\mathrm{N}$ \\
\hline UAC5 & p.G12D & $\mathrm{N}$ & p.H168Y & p.R201H & c.729delG & $\mathrm{N}$ \\
\hline UAC6 & p.G12D & $\mathrm{N}$ & $\mathrm{N}$ & p.R201H & $\mathrm{N}$ & $\mathrm{N}$ \\
\hline UAC7 & p.G12S & $\mathrm{N}$ & $\mathrm{N}$ & $\mathrm{N}$ & $\mathrm{N}$ & $\mathrm{N}$ \\
\hline UAC8 & p.G12S & $\mathrm{N}$ & $\mathrm{N}$ & $\mathrm{N}$ & $\mathrm{N}$ & $\mathrm{N}$ \\
\hline UAC9 & $\mathrm{N}$ & $\mathrm{N}$ & $\mathrm{N}$ & $\mathrm{N}$ & $\mathrm{N}$ & $\mathrm{N}$ \\
\hline UAC10 & $\mathrm{N}$ & $\mathrm{N}$ & $\mathrm{N}$ & $\mathrm{N}$ & $\mathrm{N}$ & $\mathrm{N}$ \\
\hline UAC11 & $\mathrm{N}$ & $\mathrm{N}$ & $\mathrm{N}$ & $\mathrm{N}$ & p.N129S & $\mathrm{N}$ \\
\hline UAC12 & $\mathrm{N}$ & $\mathrm{N}$ & $\mathrm{N}$ & $\mathrm{N}$ & $\mathrm{N}$ & $\mathrm{N}$ \\
\hline UAC13 & $\mathrm{N}$ & $\mathrm{N}$ & p.E258K & $\mathrm{N}$ & $\mathrm{N}$ & $\mathrm{N}$ \\
\hline UAC14 & $\mathrm{N}$ & $\mathrm{N}$ & p.M246V & $\mathrm{N}$ & p.G386D & $\mathrm{N}$ \\
\hline UAC15 & $\mathrm{N}$ & $\mathrm{N}$ & p.R248W & $\mathrm{N}$ & $\mathrm{N}$ & $\mathrm{N}$ \\
\hline UAC16 & NRAS p.G12D & $\mathrm{N}$ & p.V216L & $\mathrm{N}$ & $\mathrm{N}$ & $\mathrm{N}$ \\
\hline $\mathrm{BACl}$ & $\mathrm{N}$ & $\mathrm{N}$ & p.R248W & $\mathrm{N}$ & $\mathrm{N}$ & p.S1545X \\
\hline $\mathrm{BAC2}$ & $\mathrm{N}$ & $\mathrm{N}$ & p.R248W & $\mathrm{N}$ & $\mathrm{N}$ & $\mathrm{N}$ \\
\hline $\mathrm{BAC} 3$ & $\mathrm{~N}$ & $\mathrm{~N}$ & p.G245C & $\mathrm{N}$ & $\mathrm{N}$ & $\mathrm{N}$ \\
\hline BAC4 & $\mathrm{N}$ & $\mathrm{N}$ & p.R175H & $\mathrm{N}$ & $\mathrm{N}$ & p.E1317Q \\
\hline
\end{tabular}

Conclusions: In summary, UACs and BACs have distinct mutation profiles, which may aid in their diagnosis.
886 TERT Promoter Mutations in Urinary Bladder Adenocarcinoma Morgan Cowan, Simeon Springer, Doreen Nguyen, Diana Taheri, Gunes Guner, Maria Angelica Mendoza Rodriguez, Isabela W Cunha, Dilek Baydar, Stephania Bezerra, Christopher J VandenBussche, Yuxuan Wang, Kenneth Kinzler, Nickolas Papadopoulos, Bert Vogelstein, George J Netto. Johns Hopkins Hospital, Baltimore, MD; Isfahan University of Medical Sciences, Isfahan Kidney Diseases Research Center, Isfahan, Islamic Republic of Iran; AC Camargo Cancer Centre, Sao Paulo, Brazil; Hacettepe University, Ankara, Turkey.

Background: TERT promoter mutations (TERT-mut) have been detected in $60-80 \%$ of urothelial carcinomas. A molecular urine-based screening assay for the detection of TERT-mut is currently being pursued by our group and others. A significant number of bladder carcinomas are adenocarcinoma (ACA). The current study assesses the incidence of TERT-mut in primary adenocarcinomas of urinary bladder.

Design: A retrospective search of our institutional pathology records identified 23 cystectomy specimens with a diagnosis of Primary ACA (2000-2014). All slides were reviewed to confirm tumor type and select a representative FFPE block for mutational analysis. Adequate material for DNA testing was available in 14 cases [7 enteric type and 7 not other specified (NOS)]. Tumor areas were cored for DNA purification. Samples were analyzed with Safe-SeqS, a sequencing error-reduction technology and sequenced using Illumina MiSeq next generation sequencing technology as we previously described (Kinde, I et al. Cancer Res 2013: 367)

Results: Overall, $28.5 \%$ of primary adenocarcinoma harbored TERT-mut. Four of seven $(57 \%)$ of non-enteric (NOS) adenocarcinoma were mutation positive, while none of the enteric-type tumors harbored mutations. All 4 tumors had identical TERT-mut variant to the one we previously found in urothelial carcinomas of the bladder (g.1295228C $>\mathrm{T})$.

\begin{tabular}{|l|l|l|l|}
\hline \multirow{2}{*}{ Histologic Type } & \multirow{2}{*}{ TERT promoter mutation } & \multicolumn{2}{|l|}{ Mutation Type } \\
\cline { 3 - 4 } & & $228 \mathrm{~T}>\mathrm{C}$ & $250 \mathrm{~T}>\mathrm{C}$ \\
\hline Enteric $(\mathrm{n}=7)$ & $0(0 \%)$ & - & - \\
\hline Non-Enteric NOS $(\mathrm{n}=7)$ & $4(57 \%)$ & $4 / 4(100 \%)$ & 0 \\
\hline All $(\mathrm{n}=14)$ & $4(28.5 \%)$ & $4 / 4(100 \%)$ & 0 \\
\hline
\end{tabular}

Conclusions: Similar to urothelial carcinoma, we found a relatively high rate of TERT-mut among non-enteric type adenocarcinoma further supporting the potential utility of TERT-mut urine-based screening assay for bladder cancer including primary adenocarcinoma. The contrast in the incidence of TERT-mut between enteric and nonenteric types of bladder adenocarcinoma is intriguing and most likely reflect differences in tumorigenesis pathways among these histologic types of tumors.

\section{TERT Promoter Mutations in Squamous Cell Carcinoma of the} Bladder

Morgan Cowan, Simeon Springer, Doreen Nguyen, Diana Taheri, Gunes Guner, Maria Angelica Mendoza Rodriguez, Maria DC Rodriguez, Isabela W Cunha, Dilek Bavdar, Yuxuan Wang, Kenneth Kinzler, Nickolas Papadopoulos, Bert Vogelstein, George $J$ Netto. Johns Hopkins Hospital, Baltimore, MD; Sidney Kimmel Comprehensive Cancer Center, Baltimore, MD; Isfahan University of Medical Sciences, Isfahan Kidney Diseases Research Center, Isfahan, Islamic Republic of Iran; AC Camargo Cancer Center, Sao Paulo, Brazil; Hacettepe University, Ankara, Turkey.

Background: Previously, TERT promoter mutations have been detected in $60-80 \%$ of urothelial carcinomas, including those with focal squamous differentiation. Squamous cell carcinoma (SCC) in other organs, including, lung and cervix has not been shown to harbor high rates of TERT promoter mutations. No study has yet reported the presence of TERT promoter mutations in pure SCC of the bladder.

Design: Retrospective review of the surgical pathology archives for cystectomy specimens of pure primary bladder SCC identified 15 cases (2000-2014). All histologic slides and medical records were reviewed to confirm the diagnosis of primary SCC of the bladder. Representative tumor areas were cored

for DNA purification. Samples were analyzed with Safe-SeqS, a sequencing errorreduction technology and sequenced using Illumina MiSeq as we previously described (Kinde, I et al. Cancer Res 2013: 367)

Results: Similar to our previous findings in urothelial carcinoma, TERT promoter mutations proved highly prevalent in SCC of the bladder with mutations detected in $12 / 15(80 \%)$ tumors. Ten of $13(83 \%)$ conventional SCC revelaed the g. 1295228 $\mathrm{C}>\mathrm{T}$ TERT promoter mutation with the remaining $2(17 \%)$ tumors with papillary and sarcomatoisd features showing g.1295250C $>\mathrm{T} T E R T$ mutation variant.

\begin{tabular}{|l|l|l|l|}
\hline \multirow{2}{*}{ SCC Histologic Features } & \multirow{2}{*}{$\begin{array}{l}\text { TERT promoter } \\
\text { mutation detected }\end{array}$} & \multicolumn{2}{|l|}{ Mutation } \\
\cline { 3 - 4 } & $10(77 \%)$ & $228 \mathbf{C}>\mathbf{T}$ & $\mathbf{2 5 0} \mathbf{C}>\mathbf{T}$ \\
\hline Conventional SCC $(\mathrm{n}=13)$ & $1(100 \%)$ & 0 & $8(80 \%)$ \\
\hline SCC with Papillary Features $(\mathrm{n}=1)$ & $1(100 \%)$ \\
\hline SCC with Sarcomatoid Features $(\mathrm{n}=1)$ & $1(100 \%)$ & 0 & $1(100 \%)$ \\
\hline All $(\mathrm{n}=15)$ & $12(80 \%)$ & $2(17 \%)$ & $10(83 \%)$ \\
\hline
\end{tabular}

Conclusions: TERT promoter mutations are present in pure squamous cell carcinoma of the bladder in high rates, similar to urothelial carcinoma.This similarity lends support to shared tumorigenesis. Furthermore, a urine screening assay for bladder carcinoma based on TERT promoter mutations would detect most SCC as well as urothelial carcinomas. 
888 Human Hemochromatosis Protein (HFE) Immunoperoxidase Stain Highlights Choriocarcinoma within Mixed Germ Cell Tumors

Jesse L Cox, Geoffrey A Talmon, Scott A Koepsell. University of Nebraska Medical Center, Omaha, NE.

Background: The identification of choriocarcinoma within a germ cell tumor can have major implications for the subsequent staging and treatment of testicular neoplasms. Immunoperoxidase staining greatly enhances the speed and sensitivity of identifying occult, though clinically significant, tumor components. In mixed germ cell tumors, staining for beta-human chorionic gonadotropin ( $\beta-\mathrm{hCG}$ ) has historically been used to assess for the presence and burden of choriocarcinoma. However, current $\beta$-hCG stains produce variable, sometimes intense, staining of trophoblastic elements and surrounding tissue, interfering with and clouding the assessment of true-positive staining. Human hemochromatosis protein (HFE) is a membrane-bound mediator of iron transport expressed at high levels within human placental tissues, as determined by microarray analysis. Additionally, previous reports have demonstrated that choriocarcinoma cell lines express HFE, although in vivo expression has not been examined.

Design: HFE expression was examined by immunohistochemistry in patients with choriocarcinoma (4 samples), mixed germ cell tumors (11), and seminoma (4), to determine whether HFE could identify the trophoblastic components of choriocarcinoma, and to characterize non-specific staining by HFE. Moreover, to determine whether HFE highlighted syncitiotrophoblasts, intermediate trophoblasts and/or cytotrophoblasts, products of conception (11) were also examined. $\beta$-hCG immunohistochemical staining was used as a control.

Results: HFE consistently demonstrated cytoplasmic and membranous staining, highlighting both syncytiotrophoblasts and cytotrophoblasts within choriocarcinoma and products of conception. Mild punctate staining was seen within seminomas and germ cell tumors without choriocarcinoma upon examination for HFE. This background staining corresponded with lymphocytic infiltrate within the tumor, corroborating prior reports that HFE highlights monocytes and macrophages.

Conclusions: HFE may serve as an alternative target from $\beta$-hCG for immunoperoxidase studies when trying to detect occult choriocarcinoma.

889 Integrated Molecular Classifier Determines Transdifferentiation to Neuroendocrine Prostate Cancer

Joanna Cyrta, Himisha Beltran, Davide Prandi, Juan Miguel Mosquera, Matteo Benelli, Scott A Tomlins, Olivier Elemento, Andrea Sboner, Levi Garraway, Mark Rubin, Francesca Demichelis. Weill Cornell Medicine of Cornell University, New York, NY; University of Trento, Trento, Italy; University of Michigan, Ann Arbor, MI; Dana Farber Cancer Institute, Boston, MA

Background: Distinguishing conventional castrate-resistant prostate adenocarcinoma (CRPC-A) from poorly differentiated CRPC with neuroendocrine differentiation (CRPC-N) is important for clinical decision making. We analyzed genomic alterations, DNA methylation and gene expression profiles of CRPC-N compared to CRPC-A. This study nominated an integrated, gene expression-based signature highly specific for CRPC-N.

Design: Metastatic CRPC ( $\mathrm{n}=114$ ) with available fresh frozen material were classified based on consensus pathology guidelines. Whole exome sequencing was performed on 70 CRPC-A (51 patient [pts]) and 44 CRPC-N (30 pts) samples; RNA-seq on 34 CRPC-A (25 pts) and 15 CRPC-N (10 pts); eRRBS on 18 CRPC-A (12 pts) and 10 CRPC-N (8 pts). Integrative analysis of these data nominated a set of 70 genes with the most significant differential methylation $(\mathrm{n}=11)$, deletion/amplification $(\mathrm{n}=16)$ and/or expression status $(\mathrm{n}=38)$ and 5 additional relevant genes from previous studies. Using gene expression levels of these 70 genes, Integrated NEPC score (Pearson's correlation coefficient) represented the probability of a sample to be CRPC-N.

Results: The score correctly classified nearly all samples from this study $(>0.99$ precision and recall) and most samples from independent datasets: TCGA (all correctly classified as non-CRPC-N), International SU2C/PCF cohort (precision and recall $=0.6$ ) and our precision medicine CRPC cases (precision $=1$, recall $=0.86$ ). Interestingly, 4 independent CRPC-A cases with a high NEPC score revealed clinical and/or pathologic features of CRPC-N upon reevaluation.

Conclusions: Morphology and IHC are the current standard for CRPC-N diagnosis, but may be insufficient in atypical cases, cases of CRPC-A transitioning to CRPC-N and/or those with "small cell-like" behavior in spite of adenocarcinoma morphology. The proposed transcriptome-based signature uses a more exhaustive molecular profile for CRPC classification and could represent a valuable diagnostic and prognostic tool for patients with advanced CRPC.

890 Do African Americans with Low Risk Prostate Cancer on Needle Biopsy (Eligible for Active Surveillance), Have More Aggressive Pathology in Radical Prostatectomy Specimens Compared to Their European American Counterparts?

MHD Fayez Daaboul, Oudai Hassan, Dongping Shi, Muhammad K Alsafadi, Isaac Powell, Michael L Cher, Wael A Sakr. WSU, Detroit, MI.

Background: Previous reports indicate that low risk prostate cancer (PCA) newly diagnosed on needle biopsies $(\mathrm{Bx})$ is associated with worse prognostic parameters on radical prostatectomy specimens (RPS) in African American (AA) compared to European American (EA) patients. The objective of this study was to compare the pathological findings in RPS for patients of the two racial groups whose initial Bx findings would have qualified them to opt for enrolling in active surveillance (AS) protocol.

Design: Consecutive AA and EA patients newly diagnosed PCA at our institution whose disease was limited to $<=3$ cores of different anatomic sites, $<=50 \%$ involvement of any core and Gleason score (GS) of $<=6$ who chose to be treated by RP were included in this study. The RPS of the AA and EA patients were compared relevant to pathologic stage, GS, tumor volume and extent of anterior/apical distribution (AD), classified as prominent or absent/ minimal.

Results: One hundred and thirty two AA and 44 EA patients qualified for the study. $91 \%$ and $9 \%$ of AA patients had stages pT2 and pT3 respectively. The corresponding figures for EA were $89 \%$ and $11 \%$, ( $\mathrm{p}=\mathrm{NS}$ ). No significant differences were found in GS or tumor volume in RPS between the two groups. Twenty nine (22\%) of AA patients had prominent $\mathrm{AD}$ compared to $3(6.8 \%)$ EA patients $(\mathrm{p}=0.016)$. We found significant associations between prominent $\mathrm{AD}$ and pathologic stage and $\mathrm{GS}$ in $\mathrm{AA}$ patients ( $\mathrm{p}=0.015$ and 0.021 respectively), [Table 1].

\begin{tabular}{|l|l|l|l|l|}
\hline \multicolumn{2}{|l|}{ Pathological Parameters } & AA & EA & p value \\
\hline \multirow{2}{*}{ Stage } & $<=\mathrm{T} 2$ & $118(90.8 \%)$ & $39(88.6 \%)$ & \multirow{2}{*}{0.43} \\
\cline { 2 - 4 } & $>=\mathrm{T} 3$ & $12(9.2 \%)$ & $5(11.4 \%)$ & \\
\hline \multirow{2}{*}{ GS } & $<=6$ & $83(63.8 \%)$ & $34(77.3 \%)$ & \multirow{2}{*}{0.071} \\
\cline { 2 - 4 } & $>6$ & $47(36.2 \%)$ & $10(22.7 \%)$ & \\
\hline \multirow{2}{*}{ AD } & No & $103(78 \%)$ & $41(93.2 \%)$ & \multirow{2}{*}{0.016} \\
\cline { 2 - 4 } & Yes & $29(22 \%)$ & $3(6.8 \%)$ & \\
\hline \multirow{2}{*}{ Prominent AD } & High stage & $7(24.1 \%)$ & $0(0 \%)$ & 0.015 \\
\cline { 2 - 4 } & High GS & $16(55.2 \%)$ & $0(0 \%)$ & 0.021 \\
\hline \multirow{2}{*}{ Tumor Volume } & $<20 \%$ & $82(82 \%)$ & $28(82.4 \%)$ & \multirow{2}{*}{0.59} \\
\cline { 2 - 4 } & $>=20 \%$ & $18(18 \%)$ & $6(17.6 \%)$ & \\
\hline
\end{tabular}

Conclusions: Prominent anterior/apical tumor distribution in RPS was significantly higher in AA compared to EA patients in our study cohort of low risk PCA on Bx. Furthermore, we found a statistically significant association between AD and RPS stage and GS in AA patients. Our findings are consistent with data suggesting that PCA with AD component, often of higher GS could be missed on initial Bx and that MRI aided sampling maybe indicated to detect this anatomic component, particularly in AA patients.

891 Molecular Subtypes of Bladder Cancer and Their Signature Markers in Multi-Institutional Cohorts

Vipulkumar Dadhania, Charles C Guo, Miao Zhang, Tadeusz Majewski, Li Zhang, Jolanta Bondaruk, Shizhen Zhang, Woonyoung Choi, David McConkey, Colin Dinney, Keith Baggerly, Bogdan Czerniak. The University of Texas MD Anderson Cancer Center, Houston, TX.

Background: Recently we have proposed a molecular classification of conventional urothelial carcinoma which could be divided into two categories designated as basal and luminal. In addition, a subset of luminal cancers was characterized by upregulation of 553 regulatory genes and is referred to as

p53-like. The purpose of this project is to verify whether such subtypes can be identified in several independent cohorts of bladder cancers.

Design: Molecular subtyping was performed by analyzing gene expression profiles with previously described algorithms on the following cohorts: (1) The TCGA cohort containing 129 cases of muscle invasive tumors; (2) MDACC cohort of fresh frozen tumor tissues which included 65 superficial and 77 invasive tumors; (3) Swedish cohort containing fresh frozen tumor samples of 116 superficial and 192 invasive tumors; and (4) the second MDACC cohort of formalin-fixed paraffin-embedded tissue (FFPE) from 89 muscle invasive tumor samples. Analysis of biomarkers by immunohistochemistry (IHC)

was performed on the FFPE cohort using the parallel tissue microarray. The following markers were selected for IHC: CK18, CK20, uroplakin, GATA3, ER, and HER-2 for luminal subtype; 16 , Bcl-2, desmin, SMA, myosin, and calponin for p53-like subtype; and CK5/6, CK14, CD44, cyclinD1, p63, and vimentin for basal subtype.

Results: Two subtypes defined as luminal and basal were identified in all four cohorts. Additionally, a subset of luminal subtype showed upregulation of $\mathrm{p} 53$ regulatory genes. IHC disclosed that 12 of the 18 selected markers showed distinctive staining among molecular subtypes. Luminal cancers were characterized by the co-expression of CK20 and GATA3. The p53-like cancers were characterized by co-expression of uroplakin and Her-2. Basal cancers were characterized by co-expression of CK5/6, CK14, and CD44. Conclusions: Our study validated the hypothesis that based on gene expression profiles, bladder cancer can be classified into several subtypes which can also be identified with a limited number of IHC markers. This validation has important implications for bladder cancer diagnosis and patient management.

\section{MAGI-2 is Associated with Biochemical Recurrence in Prostate} Cancer

Stephanie N David, Shanna Arnold, Rajen Goyal, Jeffery Goldstein, Lan L Gellert, Peter E Clark, Omar Hameed, Giovanna A Giannico. Vanderbilt University Medical Center, Nashville, TN; Department of Veterans Affairs, Nashville, TN.

Background: Membrane-associated guanylate kinase, WW and PDZ domain-containing protein 2 (MAGI-2) promotes the activity of phosphatase and tensin homolog (PTEN). Recent studies suggest that dysregulation of this signaling pathway has a role in prostate carcinogenesis. We have previously demonstrated that MAGI-2 is elevated in high grade prostatic intraepithelial neoplasia (HGPIN) and prostate cancer across all Gleason grades compared to normal glandular epithelium by immunohistochemistry (IHC). Our study aims to expand upon these findings and determine whether MAGI-2 expression has prognostic utility in prostate cancer.

Design: Tissue microarrays consisting of 1-mm cores were constructed from 56 cases of radical prostatectomy to include benign prostatic glands, HGPIN, and adenocarcinoma. IHC for MAGI-2 was performed. MAGI-2 percent thresholded area (\%AREA) was quantified by image analysis. Multivariable Cox proportional hazards and logistic 
regression models were used to correlate MAGI-2 expression with clinical outcomes of survival and recurrence. Generalized Estimating Equations (GEE) with linear and logistic regression models were used to correlate MAGI-2 with core histology.

Results: Mean age and PSA at presentation were 60 years (range 41-72) and $10.8 \mathrm{ng} / \mathrm{ml}$ (range 4-80). Median time to biochemical recurrence (BCR) was 11.9 months. MAGI-2 \%AREA was inversely associated with progression from HGPIN to adenocarcinoma of low to high Gleason grade (OR, 0.98; slope, $-0.02 ; \mathrm{P}=0.005)$ and HGPIN to cancer of any Gleason score (OR, 0.97; $\mathrm{P}=0.007)$. After adjusting for race, PSA, Gleason grade, stage tumor volume, extraprostatic extension and nodal involvement, MAGI-2 \%AREA was a significant independent predictor of $\mathrm{BCR}$ ( $\mathrm{OR}, 0.93$; $95 \%$ CI, $0.87-0.99$; $\mathrm{P}=0.019$; bootstrap $\mathrm{P}=0.002$ ). These results were maintained in a more restrictive model, adjusting for Gleason grade and stage only (OR, $0.95 ; 95 \%$ CI, $0.91-0.99 ; \mathrm{P}=0.037$; bootstrap $\mathrm{P}=0.013$ ). The addition of MAGI- $\%$ AREA in both models to these standard clinical parameters improved accuracy of predicting BCR by $5.5 \%(91.8 \%$ vs. $86.3 \%)$ and $5.4 \%(82.6 \%$ vs. $77.2 \%)$, respectively.

Conclusions: These results suggest that MAGI- 2 expression is reduced during prostate cancer progression and that retention of MAGI-2 signal reduces odds of BCR. These results further substantiate MAGI-2's role in prostate neoplasia. Such findings may be used to guide treatment decisions and post-operative surveillance intervals.

893 PIM1 Expression in Prostate Cancer: Correlation with ERG Rearrangement, bcl-2 and Ki67 Index

Silvia de Muga, Joan Gil, Silvia Hernandez, Alba Font, Marta Lorenzo, Nuria Juanpere, Lluis Fumado, Lluis Cecchini, Laia Serrano, Josep Lloreta. Universitat Pompeu Fabra, Barcelona, Spain; Hospital del Mar-Parc de Salut Mar-IMIM, Barcelona, Spain; Autonomous University of Barcelona, Barcelona, Spain.

Background: PIMI has been related to genomic instability, $A K T$ activation and $E R G$ expression in prostate cancer (PCa), but data on the relationship between these two genes and tumor features is limited. The aim of this study has been to analyze the relationship between $E R G$, PIMI, bcl-2 and Ki67 expression, and their relationship with Gleason score (GS).

Design: PIM1 expression was assessed by qRT-PCR in 55 frozen PCa and 3 non-tumor samples (Parc de Salut MAR Biobank - MARBiobanc) of Barcelona, Spain. In addition, PIM1 (ab75776, Abcam) immunohistochemistry (IHC) was performed in 9 TMA blocks containing samples from 260 radical prostatectomy specimens. The relationship with ERG, bcl-2 expression and Ki67 labelling index, as well as the association with Gleason score (GS) was statistically assessed.

Results: PIM1 mRNA was overexpressed in $43.6 \%$ (24/55) of prostate tumors. It was associated to $\mathrm{G} \geq 8$ prostate tumors (Fisher's Exact test, $\mathrm{p}=0.012$ ) as $16.7 \%(3 / 18)$ of $\mathrm{GS}=6,50 \%(11 / 22)$ of $\mathrm{GS}=7$ and $66.7 \%(10 / 15)$ of $\mathrm{GS} \geq 8$ tumors showed PIMI overexpression. Almost 84\% (218/260) of the cases overexpressed PIM1 protein (96 had low expression and 122 had high expression). According to GS, 79.8\% (142/178) of GS $\leq 7$ and $92.7 \%(76 / 82)$ of GS $\geq 8$ had PIM1 protein overexpression, thus the latter was associated to $\mathrm{GS} \geq 8$ (Chi-square test, $\mathrm{p}=0.008$ ). We found a significant association between higher PIM1 protein levels and ERG expression, as 57.1\% (141 cases) were concordant (Fisher Exact test; $p=0.001$ ). ERG positive tumors correlated with higher levels of PIM1 and Bcl-2 (Student's t-test, $\mathrm{p}<0.01$ for PIM1 and $\mathrm{p}=0.03$ for Bcl-2). Both PIM1 and Bcl-2 weakly correlated with higher Ki67 indexes (Pearson's $r=0.19, p<0.01$ for PIM1 and Spearman's rho=0.22, $p<0.01$ for Bcl-2). ERG also showed a marginally significant association with Ki67 levels (Student's t-test $\mathrm{p}=0.09$ ). Conclusions: PIM1 overexpression is associated to the more aggressive PCa, and it is related to ERG protein overexpression. The association of these two genes with higher bcl-2 expression and Ki67 index suggests that they may have an effect on apoptosis and tumor cell proliferation.

Supported by FIS/ Instituto de Salud Carlos III/ FEDER/PI12/01426, Spanish Ministry of Health and personal AECC (Asociación Española Contra el Cáncer) grant.

894 Urinary Exosomes with TMAs Validation: The Mirror of Prostate Alterations

Ines De Torres, Tamara Sequeiros, Dirk Michiel Pegtel, Eduard Sabido, Marina Rigau, Iolanda Garcia-Grau, Jordi Temprana-Salvador, Juan Morote, Santiago Ramon y Cajal, Mireia Olivan. Vall d'Hebron University Hospital, Universitat Autònoma de Barcelona (UAB), Barcelona, Spain; Centre de Regulació Genòmica (CRG), Barcelona, Spain; Vall d'Hebron Institut de Recerca (VHIR), Barcelona, Spain; Vall d'Hebron University Hospital and Autònomous University of Barcelona (UAB), Barcelona, Spain; VU University Medical Center, Cancer Center Amsterdam, Amsterdan, Netherlands. Background: New easy and reliable biomarkers for prostate cancer diagnosis (PCa) would be desirable to identify those patients who could avoid overtreatment. Because of the location of the prostate in direct contact with the urethra, desquamated cells and molecules secreted, including exosomes like vesicles (ELV), are present in human urine. The urine exosomes have been recently described as carriers of genetic information and a potential source of new cancer biomarkers. The aim of this study is to identify protein biomarkers in urine exosomes for early non-invasive detection and stratification of PCa in order to verify if these proteins could reflect in urine what is happening in the prostate gland.

Design: Protein biomarker candidates for PCa were initially identified from a discovery phase done in urinary exosomes, isolated by ultracentrifugation from urine obtained after digital rectal examination. A label-free LC-MS/MS protein quantitation was performed on 24 urine samples: 8 benign samples, 8 low-risk PCa samples, and 8 high-risk PCa samples. Proteins significantly changing in abundance were selected for further reaction monitoring (SRM) validation 53 urinary exosomes samples from PCa patients and 54 from benign counterparts. Subsequently IHC in TMAs prostate cancer was performed to validate the most promising candidates.
Results: 1673 proteins were identified including PSA, PSMA, and ACPP. A panel of 64 candidates was selected for validation by SRM. Ultimately a profile of 2 novel urinary exosomes-associated protein biomarkers was identified, as well as a profile of 5 proteins able to significantly distinguish between high and low risk patients validated on TMA samples.

ADVS and TGM4 were the most significant proteins for discriminating PCa and normal prostate (p-value $<0.001 ;$ ROC value 0,75 and 0.77 respectively).

Conclusions: The proteomic studies in urine exosomes could reflect what is happening in the prostate gland. Our results support that the biomarkers obtained, could be possible good candidates in early diagnosis. Furthermore these results could help decisionmaking in the personalized treatment of prostate cancer patients.

\section{PIK3CA, MET and TP53 Gene Mutations in Penile Cancer}

Jasreman Dhillon, Philippe E Spiess, Elena Ryzhova, Yin Xiong, Julio Pow-Sang, Anthony M Magliocco. Moffitt Cancer Center, Tampa, FL.

Background: Penile cancer is a rare but aggressive disease. HPV is a known factor in this tumor and is associated with improved outcome. Little information is known about the genetic mutations involved and their prognostic value. This information is useful for understanding the molecular heterogeneity and possibilities of selecting targeted therapy. Design: We selected 20 cases (HPV + 11; HPV- 9) of penile squamous cell carcinoma. DNA was extracted from formalin fixed paraffin embedded tumors and subjected to PCR amplification. Tumor tissue was screened for more than 250 mutations involving 26 oncogenes and tumor suppressor genes. We also investigated if there were driver mutations detectable.

Results: Mutations were identified in 9 of 20 cases $(45 \%)$. PIK3CA was the most common ( $\mathrm{n}=7$ ) with PIK3CA_E545K mutation present in 6 cases and PIK3CA_H104L mutation in one case. 5 of $11 \mathrm{HPV}$ positive cases had mutations and all involved PIK3CA E545K. Two cases had MET N375S mutation. Both of these were HPV negative cases (well differentiated squamous cell carcinoma and verrucous carcinoma). A poorly differentiated squamous cell carcinoma had mutation involving TP53 R248Q.

\begin{tabular}{|l|l|l|}
\hline Mutation Detected & HPV + Cases & HPV - Cases \\
\hline None & 6 & 5 \\
\hline PIK3CA & 5 & 2 \\
\hline MET & 0 & 2 \\
\hline TP53 & 0 & 1 \\
\hline
\end{tabular}

Presence of mutations was not significantly associated with HPV status (chi square test, $\mathrm{p}=1.0000), \mathrm{p} 53(\mathrm{p}=1.0000), \mathrm{p} 16(1.0000)$ and HPV ISH $(\mathrm{p}=0.6843)$. Patients without mutations, 16 positive and HPV positive status had a better chance of overall survival, but did not reach significance value $(p<0.05)$. Patients without PIK3CA E545K and MET mutations had better overall survival but did not reach significance value (log rank test, $\mathrm{p}=0.2862$ and 0.3373 ).

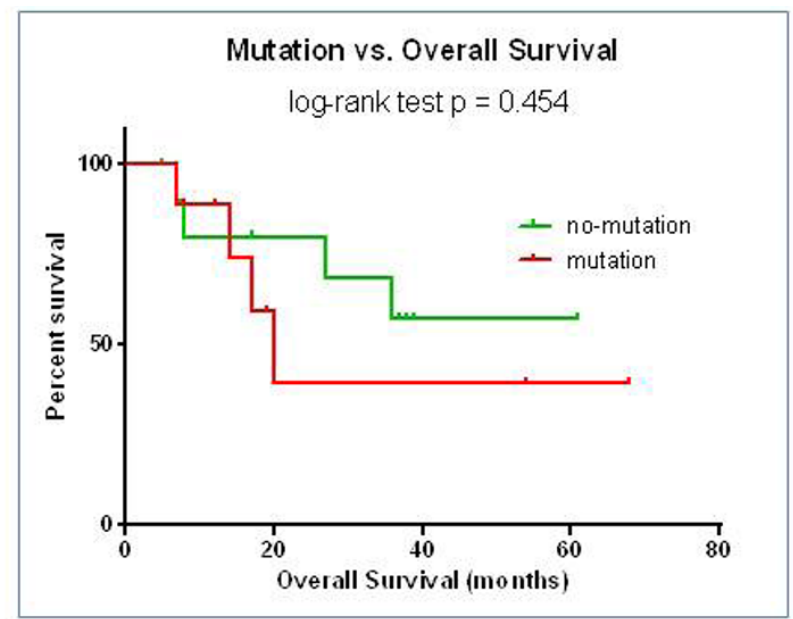

Conclusions: 1. Multiple mutations were detected with PIK3CA being the most common, similar to cervical cancer

2. This is a potentially targetable pathway for future therapy

3. Why PIK3CA mutations are common in HPV associated cases is a potential area of future investigation

896 Identification of Urinary MiRNAs for the Assessment of Small Renal Masses

Ashley Di Meo, Henriett Butz, Maria D Pasic, Michael Jewett, Alexander Romaschin, Eleftherios P Diamandis, George M Yousef. Li Ka Shing Knowledge Institute of St. Michael's Hospital, Toronto, ON, Canada; The Advanced Centre for Detection of Cancer at the Lunenfeld-Tanenbaum Research Institute of Mount Sinai Hospital, Toronto, ON, Canada; University of Toronto, Toronto, ON, Canada; Princess Margaret Hospital, University Health Network, University of Toronto, Toronto, ON, Canada.

Background: Renal cell carcinoma (RCC) is the most common malignancy of the adult kidney. Many RCCs are incidentally discovered small renal masses (SRMs) that measure $\leq 4 \mathrm{~cm}$ in diameter (stage Tla tumors). SRMs can either be benign tumors or renal cell carcinoma (RCC-SRMs). Moreover, RCC-SRMs can either be progressive or non-progressive. Distinction between these entities can only be done using invasive 
biopsy with limited success. Currently, all SRMs are treated by nephrectomy due to the lack of biomarkers that can predict behavior. Discovery of non-invasive urinary prognostic biomarkers can significantly improve SRM management.

Design: We performed a global screen of 754 urinary miRNAs by RT-PCR in patients with progressive and non-progressive clear cell RCC-SRMs in addition to patients with benign (oncocytoma) renal lesions. Bioinformatics analysis was performed to identify targeted pathways of dysregulated miRNAs.

Results: Analysis shows that miRNAs are differentially expressed in urine from patients with benign, progressive and non-progressive RCC-SRM lesions. Combinations of miRNAs were shown to have better discrimination between benign in addition to progressive and non-progressive renal lesions. From the list of miRNAs identified to have significant differential expression between benign, progressive and non-progressive lesions, many were previously reported in cancer. Common cancer pathways predicted to be targeted by identified miRNAs, include the MAPK $\left(\mathrm{p}=3.0 \times 10^{-15}\right)$ and PI3K-Akt $\left(p=9.0 \times 10^{-6}\right)$ signaling pathways. In addition, a number of pathways identified were previously found to be linked to $\mathrm{RCC}$, including the insulin signaling pathway ( $\mathrm{p}=$ $\left.3.5 \times 10^{-7}\right)$ and VEGF signaling pathway $(\mathrm{p}<0.0001)$.

Conclusions: Taken together, dysregulated miRNAs represent potential prognostic biomarkers able to distinguish between patients with benign, progressive, and nonprogressive renal lesions. Moreover, using bioinformatics analysis, we identified a number of pathways targeted by candidate miRNAs.

\section{Growth Pattern Analysis in Low Grade Clear Cell Renal Cell} Carcinomas: Prognostic Value and Biologic Significance

Salvador Diaz-Cano, Russel Sutherland, Jane Moorhead, Alfredo Blanes, Richard Dobson. King's College Hospital/King's College London, London, United Kingdom; University of Malaga School of Medicine, Malaga, Spain.

Background: The Pan-Cancer Analysis Project aimed to identify the genomic changes in cancer types from the Cancer Genome Atlas (TCGA). The meaning of architectural features in clear cell renal cell carcinomas (ccRCC) by Fuhrman grade (FG) has not been investigated at clinic-pathologic or genetic levels in this set.

Design: A systematic evaluation of 401 ccRCC included: microscopic satellites, heterogeneous clones, FG (1-4), confluent necrosis, spindle cell presence, primary and secondary growth pattern (tubular-nested-thick trabecular-solid), tumor infiltrating lymphocytes, stromal reaction (none/myxoid/desmoplastic), and edges (pushing/ infiltrative). A combined nuclear-architectural grade (CNAG) defined 3 subgroups: low nuclear(N)-architectural(A) grade, low N-high A grade, and high N-A grade; lowintermediate-high CNAG, respectively. Clinical data were also collected (gender, age, and stage). Whole-exome sequencing was performed on tumor and normal tissues from ccRCC available at the TCGA. We used a Random Forest machine learning approach comparing low FG (1-2, 174 cases) vs. high FG (3-4, 139 cases) grade.

Model Analysis

- Data was retrieved from the Pan-Cancer Analysis repository

- Functional somatic mutations unique to tumors were identified and represented as samples $\mathrm{x}$ genes mutation matrix (mutated $=1$, non-mutated $=0$ ).

- Pairwise Random Forest models were built for the low-intermediate-high CNAG subgroups

- Variable selection using Fisher's Exact test was conducted to reduce the number of predictors with 50 fold cross-validation design.

Random Forest models were based on the training set using the caret package in R, and predictive accuracy measured in an independent test set.

Results: Complete histological-molecular data were available in 313 cases (120 low CNAG, 54 intermediate CNAG, 139 high CNAG). Intermediate CNAG were significantly different from low CNAG regarding microscopic satellites $(48 / 54 \mathrm{vs}$. $105 / 120, \mathrm{P}=0.007)$, and morphologically heterogeneous clones (43/54 vs. $112 / 120$, $\mathrm{P}<0.001)$. The age, gender, proteins and variants model performed with an AUC of 0.803 and the predictive features for intermediate-high $\mathrm{CNAG}$ were: TP53 (OR $=8.940, \mathrm{P}<$ 2.0E-16), PIK3CA (OR = 0.239, $\mathrm{P}=4.75 \mathrm{E}-05)$, PIK3R1 $(\mathrm{OR}=0.235, \mathrm{P}=1.72 \mathrm{E}-03)$. Conclusions: Intermediate CNAG clear cell RCCs are distinctive neoplasms, morphologically heterogeneous and angioinvasive malignancies progressing through TP53 and PIK3 pathways, which offers options for targeted therapy.

898 Potential Pitfalls in the Utility of Programmed Death-1 Expression in the Prediction of Prognosis in Patients with Metastatic and Non-Metastatic Clear Cell Renal Cell Carcinoma: An Analysis of 72 Cases

Michelle DiMarco, Rishi $R$ Sekar, Dattatraya Patil, Viraj A Master, Adeboye O Osunkoya. Emory University School of Medicine, Atlanta, GA.

Background: Clear cell renal cell carcinoma (CCRCC) is the most common subtype of renal cell carcinoma and is well known to have a propensity to metastasize. Programmed Death-1 (PD-1) is known to play a pivotal role in regulating host immune responses in a variety of malignances. However, the correlation between PD-1 expression in tumor infiltrating lymphocytes of primary non-metastatic CCRCC and matched primary and metastatic CCRCC has not been well characterized, especially regarding PD-1 expression at the tumor-parenchymal interface.

Design: A search was made through our Urologic Pathology database for nephrectomy cases of primary CCRCC without clinical or pathologic evidence of metastasis. In addition, nephrectomy cases of matched primary CCRCC and corresponding lung metastasis were also obtained. The slides were re-reviewed and sections of blocks with tumor and adjacent benign renal parenchyma were obtained for PD-1 immunohistochemical stains on all cases. Tumors were considered negative for PD-1 when less than $1 \%$ of the tumor had positive expression.

Results: Fifty three cases of primary CCRCC without clinical or pathologic evidence of metastasis were analyzed. Of these, 24/53 (45\%) were positive for PD- 1 . Nineteen nephrectomy cases of matched primary CCRCC and metastatic CCRCC to the lung were also obtained. Of these, $13 / 19(68 \%)$ primary tumors exhibited PD-1 positivity and $11 / 19(57 \%)$ of the corresponding metastatic nodules were PD-1 positive. Only 9/19 (47\%) matched pairs were positive for PD-1 in both the primary and metastatic tumor. A pattern of positivity concentrated at the interface between tumor and benign renal parenchyma was observed in 18/72 (25\%) of all primary tumors.

Conclusions: PD-1 expression was higher in the primary tumors of patients with metastatic CCRCC versus those without metastatic CCRCC. PD-1 expression in the primary tumor does not always match the corresponding metastatic tumor. The potential intratumoral heterogeneity of PD-1 expression in CCRCC (tumor-parenchymal interface versus tumor away from the interface) implies that its utility as a predictive biomarker should be done with caution, especially in needle core biopsies of kidney tumors.

899 Metastatic Potential of Gleason Score $\leq 7$ Tumor at Radical Prostatectomy (RP) Using the 2014 Modified Gleason Score: Effect of High Grade Tertiary Patterns

Mairo L Diolombi, Jonathan I Epstein. Johns Hopkins Hospital, Baltimore, MD.

Background: In studies prior to the 2005 Modified Gleason Score (GS), metastases to pelvic lymph nodes (LN) were occasionally seen with GS6 at RP. Using the 2005 Modified GS, several studies have shown that pure GS6 does not spread to pelvic LN. What is not known is using the 2014 Modified GS what is the risk of pelvic LN metastases with GS6 with $<5 \%$ pattern 4 (tertiary pattern 4 -TP 4 ) vs. $3+4$ vs. $3+4$ with $<5 \%$ pattern 5 (tertiary pattern 5 -TP5) vs. $4+3$ vs. $4+3$ with $<5 \%$ pattern 5 (TP5).

Design: We searched our RP database (2005-2014) for cases of GS $\leq 7$. Since 2005, we have graded all cribriform cancer as Gleason pattern 4 consistent with the 2014 Modified GS. All prostatectomies were embedded in entirety and included pelvic LN dissections. A total of 7906 cases were identified, of which 75 had a positive LN.

Results: The incidences of metastases at RP for $3+3,3+3$ with TP $4,3+4,3+4$ with TP $5,4+3$, and $4+3$ with TP5 were $0 \%, 0.2 \%, 0.7 \%, 0.8 \% 4.5 \%$ and $6.1 \%$ respectively.

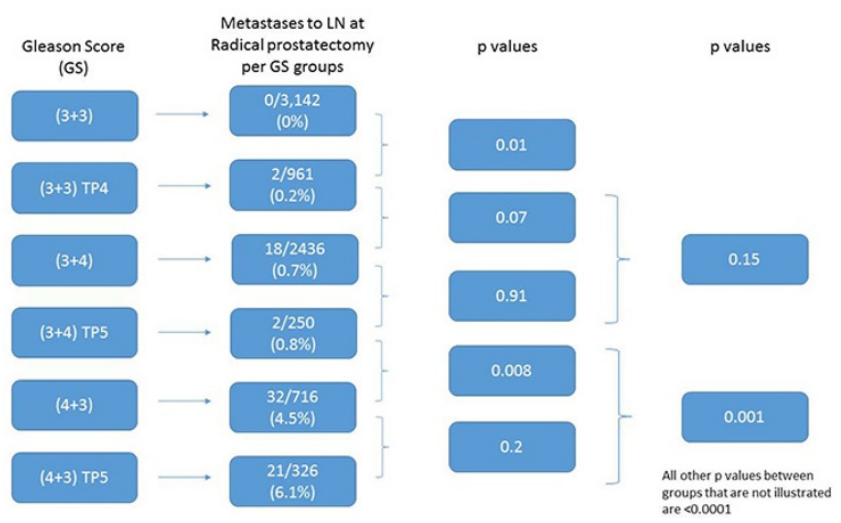

There was no statistically significant difference in risk for LN metastases at RP for men with $3+3$ with TP 4 vs. $3+4$, and for men with $3+4$ vs. $3+4$ with TP5. There was a statistically significant difference in risk for $\mathrm{LN}$ metastases at RP for men with $3+3$ vs. $3+4$.

Conclusions: This study supports our previous finding that men with $3+3$ at RP have no risk of LN metastases. These findings also support the 2014 revisions to RP grading where $3+3$ with TP4 (2005 Modified Grading System) is now considered 3+4 with recording percent pattern 4 , since TP4 increases the risk of LN metastases. It also supports keeping $3+4$ with $<5 \%$ pattern 5 , and $4+3$ with $<5 \%$ pattern 5 as $3+4$ and $4+3$, respectively, with a notation of the higher tertiary grade patterns. The less than $5 \%$ higher grade component did not increase the risk of LN metastases within a given grade. Finally, our findings highlight that $3+4$ and $4+3$ are distinct groups with respect to LN metastases and should not be combined under the umbrella designation of GS 7 as is often the case in the literature.

900 Analysis of Urothelial Carcinomas with Grade Heterogeneity: Evidence for an Early Role of CDKN2A Deletions in the FGFR3 Pathway Michelle Downes, Berber Weening, Bas WG van Rhijn, Cherry L Have, Kilian M Treurniet, Theo Van Der Kwast. Sunnybrook Health Sciences Centre, Toronto, ON, Canada; University Health Network, Toronto, ON, Canada; Erasmus Medical Centre, Rotterdam, Netherlands; Netherlands Cancer Institute, Amsterdam, Netherlands; Academic Medical Centre, Amsterdam, Netherlands.

Background: The current dual pathway model of urothelial carcinogenesis does not explain grade and stage progression in patients with initial low grade, non-muscle invasive (NMI) urothelial carcinomas. Previous work has demonstrated FGFR3 mutations to be a hallmark of the low grade pathway with progression to muscle invasive cancer occurring when FGFR 3 mutant tumours exhibit a homozygous $C D K N 2 A$ deletion. The phenomenon of grade heterogeneity in urothelial carcinoma represents the morphologic manifestation of grade progression. We hypothesized that within low grade areas ofsuch cancers, molecular evidence ( $C D K N 2 A$ deletion, increased MIB1 labelling and p53 overexpression) heralding grade progression would be present. Design: A retrospective search of the laboratory information system identified nineteen NMI urothelial carcinomas showing grade heterogeneity $(<20 \%$ high grade foci). Slides were scanned (Omnyx ${ }^{\text {TM }}$ VL4, 40X) and low and high grade foci digitally annotated. Immunohistochemistry for p53 (Leica) and MIB-1 (DAKO) was performed. CDKN2A deletions were assessed using multicolour FISH. FGFR3 mutation status was analysed using a SNaPshot PCR assay. 
Results: Among these 19 urothelial carcinomas 10 carried a FGFR3 mutation, and a homozygous $C D K N 2 A$ deletion in low grade areas was identified in $8 / 9(88 \%)$ technically suitable cases. Five of them represented a non-invasive (pTa) urothelial carcinoma. The homozygous $C D K N 2 A$ deletion was also found in low grade areas in 5/9 FGFR3 wild type urothelial carcinomas. Increased MIB-1 expression was seen in low grade areas of $12 / 19$ and in high grade areas of $17 / 19$ cases. In contrast, p53 staining was increased in only $1 / 19$ low grade and in $7 / 19$ high grade areas. Increased MIB-1 and p53 staining was noted in the low grade areas of 4 of 10 and 1 of 10 FGFR3 mutant cases, respectively.

Conclusions: Firstly, the elevated MIB-1 score in low grade foci compared to p53 suggests that MIB-1 is a better predictor of an associated high grade component. Secondly, that particularly in the FGFR3 mutant pathway homozygous deletions of $C D K N 2 A$ occur early, prior to grade and stage progression. Therefore, $C D K N 2 A$ deletions may serve as a prognostic biomarker in low grade urothelial carcinomas.

\section{The Histological Predictors of Metastasis and Chemotherapy/} Radiotherapy Response in Pure Classic Seminoma

Linnea Duke, Zari Dastani, Bader AlSaikhan, Armen Aprikian, Fadi Brimo. McGill University Health Center, Montreal, QC, Canada.

Background: A small minority of testicular pure classic seminomas (CS) behave aggressively and respond poorly to chemotherapy and/or radiation (CT/RT) when given in the context of retroperitoneal lymphadenopathy. We herein examine 47 consecutive radical orchiectomies of pure $\mathrm{CS}$ with clinical follow-up in order to determine whether certain pathological parameters are associated with metastases and advanced clinical stage and/or poor response to CT/RT.

Design: Only included were cases with radiological and oncological follow-up of at least one year. Full response to CT/RT was defined as residual tumor mass $\leq 1 \mathrm{~cm}$. Detailed histological evaluation was undertaken and the captured parameters were correlated with presence/absence of metastasis, and with response to CT/RT when given. Results: 19 patients had concurrent or subsequent lymph node metastases. CT was given in 15 and RT in 6 patients while 26 patients remained on surveillance without additional treatment. The following histological features were significantly associated with metastasis on univariate analysis: tumor size $>4 \mathrm{~cm}(\mathrm{p}=0.0001)$, tumor necrosis $(\mathrm{p}=0.003)$, lymphovascular invasion $(\mathrm{p}=0.004)$ and invasion of the rete testis $(p=0.002)$, hilar soft tissue $(p=0.001)$ and spermatic cord $(p=0.001)$. In contrast, tumor multicentricity $(\mathrm{p}=1)$, areas of tumor regression $(\mathrm{p}=0.4)$ and invasion of the tunica albuginea $(\mathrm{p}=0.1)$ were not significantly associated with metastasis. A logistic regression stepwise model with the two most prognostic parameters (lymphovascular invasion + tumor size $\geq 4$ ) predicted the presence of metastasis with an accuracy of $87 \%$. None of the histological features was significantly predictive of the response to CT/RT.

Conclusions: This study shows that tumor size $\geq 4$ and invasion of the rete testis and hilar soft tissue are important predictors of metastases in pure CS, and that their inclusion should likely be considered in the testis TNM staging system. On another hand, response to $\mathrm{CT} / \mathrm{RT}$ in CS cannot be reliably predicted based on the primary tumor characteristics.

902 Pathologic Correlation of Targeted Sampling in Patients with 3D Magnetic Resonance Imaging/Transrectal Ultrasound Fusion Biopsies

Zachary J Dureau, M Andrew Toussaint, Adeboye O Osunkoya. Emory University School of Medicine, Atlanta, GA.

Background: Transrectal ultrasound-guided biopsies (TRUS-GB) have traditionally been used to screen for prostate cancer (PCa). Some recent studies have also demonstrated the potential advantage of targeted magnetic resonance imaging-guided biopsies (TMRI-GB). More recently, a 3D method fusing these two modalities, known as a magnetic resonance imaging/transrectal ultrasound fusion biopsy (3D TMRI/ TRUS-FB), has been utilized. This approach crafts three dimensional images while combining the systematic (but non-targeted) sampling approach of TRUS-GB with the high quality targeted approach of TMRI-GB. There are very limited studies in the literature correlating lesions targeted by suspicion and final pathologic findings when using TMRI/TRUS-FB.

Design: A search was made through our Urologic Pathology files for prostate needle core biopsies that were obtained via 3D TMRI/TRUS-FB at our institution.

Results: Nineteen cases were identified. Mean patient (PT) age was 66 yrs (range: 45-78 yrs). One hundred eighty-eight total cores were sampled, with an average of 10 cores per procedure (range: 3-20 cores). One hundred twenty-two cores $(65 \%)$ were considered high suspicion (HS) and 66 cores (35\%) were considered low or intermediate suspicion (LIS). Following pathologic interpretation, 10/19 (53\%) cases were negative for malignancy. Ninety-six cores were from these cases. Fifty-one cores were considered HS and 45 cores were considered LIS. Nine of $19(47 \%)$ cases were found to have PCa. Ninety-two cores were from PCa cases. Seventy one of $92(77 \%)$ cores were sampled as HS, with 24 (34\%) of these being positive for PCa. Twenty one of $92(23 \%)$ cores were samples as LIS, with $5(24 \%)$ of these being positive for PCa. The Gleason score (GS)/Grade Groups (GG) in this PTs were as follows: $2 / 9(22 \%)$ PTs had a GS of $3+3=6$ (GG1), 3/9 (33\%) PTs had a GS of $3+4=7$ (GG2), 3/9 (33\%) PTs had a GS of $4+3=7$ (GG3), 1/9 (12\%) had a GS of 4+5=9 (GG5).

Conclusions: In our study, we found that 3D TMRI/TRUS-FB has a $77 \%$ sensitivity and $47 \%$ specificity for detecting the presence of $\mathrm{PCa}$. Given the superior image quality and comparatively good sensitivity, 3D TMRI-TRUS-GB appears to be a relatively effective method for the diagnosis of PCa. It is highly conceivable that $3 \mathrm{D}$ TMRITRUS-GB may also play a critical role in active surveillance and tumor mapping of patients with known $\mathrm{PCa}$.
903 Maspin Deficiency Promotes Prostatic Intraepithelial Hyperplasia Sijana H Dzinic, Ye-Shih Ho, Rodrigo Valdivia-Fernandez, M Magarida Bernardo, Shijie Sheng, Wael A Sakr. WSU, Detroit, MI.

Background: The role of tumor suppressor maspin in the genesis and pathogenesis of prostate gland has been implicated. In particular, maspin is down regulated in prostate cancer. Recently we also showed that the expression of maspin in prostate cancer cells can avert the cancer cell stemness towards differentiation and render tumor cells more sensitive to therapeutic agents. To definitively address the function of maspin in prostate epithelial cells, a genetic model is desired. Unfortunately, two earlier attempts to generate maspin knockout mouse generated controversial results. This issue needs to be resolved for future clinically relevant study of maspin.

Design: We generated a conditional maspin knockout (maspin KO) mouse model, by deleting exon 4 of the maspin gene that encompasses three critical exterior domains of the maspin protein. The ablation strategy also includes the generation of a frameshift. To achieve early and complete maspin KO, we crossed the heterozygous C57BL-6

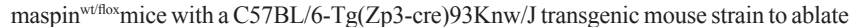
maspin exon 4 during oogenesis. The mouse prostate tissues were harvested from 6-week and 20-week old mice, respectively. For the histopathological evaluation of the mouse prostate tissues, we focused on the dorsolateral prostate, which is believed to closely resemble the peripheral zone of human prostate where $\mathrm{PCa}$ arises.

Results: Homozygous maspinKO mice, both male and female, are viable and are born in a normal Mendelian distribution with respect to other genotypes. Mouse maspin in postnatal wild type mice is expressed in an epithelial-specific manner. Interestingly, maspin KOmice are significantly underweight by approximately $20 \%$, as compared to gender and age-matched wild type mice. Maspin KO dorsolateral prostate spontaneously developed prostatic intraepithelial hyperplasia lesions with budding intraluminal projection and a degree of nuclear enlargement. There is a lack of epithelial polarity, cellcell adhesion and cell/matrix interaction. In addition, the stroma is more fibromuscular than that in wild type mice.

Conclusions: Maspin is critical in the maintenance of prostate epithelial homeostasis. Our results support a tumor suppressive role of maspin, and the utility of the maspinKO model for prostate cancer-related studies.

904 Radical Prostatectomy Findings in the Post-PSA Screening Era Liz Edmund, Andres Matoso. Rhode Island Hospital and Brown University, Providence, RI.

Background: Serum prostate-specific antigen (PSA) levels had been widely used for screening of prostate adenocarcinoma until May of 2012 when the U.S. Preventive Services Task Force (USPSTF) recommended against it based on concern for harm caused by screening and over treatment of clinically insignificant prostate cancer. We aimed to study changes in radical prostatectomy findings after the issue of USPSTF recommendation against PSA-based screening

Design: We retrieved and reviewed all consecutive RP performed at our institution immediately after the USPSTF issued its recommendation against PSA-based screening for prostate adenocarcinoma (5/2012) until 5/2015 (3 years). The control group included all RPs in the 3 years immediately before the USPSTF recommendation. Results: The results are summarized in Table 1.

\begin{tabular}{|l|l|l|l|}
\hline & $\begin{array}{l}\text { Post PSA-screening } \\
(\mathrm{n}=311)\end{array}$ & $\begin{array}{l}\text { Pre PSA-screening } \\
(\mathrm{n}=476)\end{array}$ & P value \\
\hline Age average years (range) & $60(38-81)$ & $61(42-79)$ & NS \\
\hline Gleason score No. $(\%)$ & & & \\
\hline 6 & $38(12.22 \%)$ & $116(24.35 \%)$ & $<0.01$ \\
\hline $3+4=7$ & $167(53.70 \%)$ & $255(53.57 \%)$ & NS \\
\hline $4+3=7$ & $64(20.58 \%)$ & $64(13.45 \%)$ & 0.01 \\
\hline $\begin{array}{l}6 \text { with tertiary } 4 \text { or higher } \\
\text { No. (\%) }\end{array}$ & $13(4.18 \%)$ & $20(4.20 \%)$ & NS \\
\hline 8 or above & $41(13.18 \%)$ & $40(8.40 \%)$ & 0.04 \\
\hline Organ confined & $195(62.70 \%)$ & $340(71.43 \%)$ & 0.01 \\
\hline Extraprostatic extension (focal) & $63(20.26 \%)$ & $99(20.80 \%)$ & NS \\
\hline $\begin{array}{l}\text { Extraprostatic extension } \\
\text { (established) }\end{array}$ & $53(17.04 \%)$ & $38(7.98 \%)$ & $<0.01$ \\
\hline Positive margins & $42(13.50 \%)$ & $115(24.16 \%)$ & $<0.01$ \\
\hline Seminal vesicle involvement & $23(7.40 \%)$ & $27(5.67 \%)$ & NS \\
\hline Lymph node involvement & $7(6.36 \%)$ & $6(3.70 \%)$ & NS \\
\hline Pathologic stage pT3 or above & $119(38.26 \%)$ & $139(29.20 \%)$ & 0.01 \\
\hline
\end{tabular}

Conclusions: Within a single institution, after the USPSTF recommendation against PSA-screening, the total number of RPs decreased by $35 \%$ and the number of pure Gleason score 6 at RP decreased significantly. At the same time, the post-PSA screening group had significantly higher proportion of Gleason score $4+3=7$ or above, and a significantly higher proportion of established extraprostatic extension. There was no difference in the proportion of very adverse findings including seminal vesicle invasion or lymph node involvement. Although these findings are of a limited population, they may be representative of a national trend and suggest that while many patients with clinically insignificant prostate cancer could have avoided the adverse effects of screening and treatment; a higher proportion of men undergo RP with more advanced disease and more adverse findings. 
905 Clinicopathologic Features and Outcomes of Anterior-Dominant Prostate Cancer (APCa): Implications for Diagnosis and Treatment

Sara Falzarano, Ming Zhou, Jesse K McKenney, Christopher G Przybycin, Eric A Klein, Cristina Magi-Galluzzi. Cleveland Clinic, Cleveland, OH; NYU, New York, NY. Background: An increasing number of APCa has been reported in recent years. APCa are difficult to detect by digital rectal examination (DRE), poorly visualized on imaging, and require more biopsy sessions to diagnose. Although reported to have lower Gleason scores (GS) and rates of extraprostatic extension (EPE), at least one study reported higher tumor volumes (TV) and higher PCa specific mortality rates in patients with APCa. We herein describe clinicopathological features of a series of APCa with emphasis on clinical outcomes.

Design: We searched our radical prostatectomy (RP) database for cases with an anteriordominant index tumor. Pre-operative clinical [age, initial PSA (iPSA), race, family history (FH), DRE findings], pathologic [GS, pathologic stage (pT), margin status, lymph node status $(\mathrm{pN})$, greatest tumor dimension (GTD), TV], and post-operative follow up (FU) data were collected in an IRB approved database. Biochemical failure (BF) was defined as PSA rise $>0.2 \mathrm{ng} / \mathrm{mL}$ on two consecutive measurements. Cases with history of neoadjuvant therapy or no available FU were excluded.

Results: Of $1580 \mathrm{RP}$ with available map reconstruction by expert GU pathologists, 150 $(9 \%)$ contained APCa. Upon review of medical records, 16 cases were excluded. Mean age and median iPSA of remaining patients $(\mathrm{n}=134)$ were 59 years and $6.43 \mathrm{ng} / \mathrm{mL}$, respectively. Most patients $(89 \%)$ were Caucasian. A positive FH was seen in 41 (31\%) patients. DRE was negative in $87 \%$. GS was 6 in $13 \%, 3+4$ in $61 \%, 4+3$ in $13 \%, 7$ with tertiary pattern 5 in $5 \%, 8$ in $2 \%$, and 9 in $5 \%$. Median GTD was $23 \mathrm{~mm}$; median TV was $1.8 \mathrm{cc} ; 57 \%$ of tumors were organ confined (pT2); $43 \%$ had EPE (pT3a). Margins of resection were positive in $58(43 \%)$ tumors, of which $22(38 \%)$ were otherwise pT2. Two (3\%) of 60 patients with lymph node dissection were pN1. Mean FU was 23 months $(1-120)$. BF was reported in $7(5 \%)$ patients with median time to failure of 9 months $(0-50)$. Bone metastasis was present in two patients with $\mathrm{BF}$, one of which died of disease at 116 months. No local failure was documented by either CT scan or biopsy. All patients with BF had positive margins and showed significantly higher iPSA ( 8.0 vs. $17.3 \mathrm{ng} / \mathrm{mL}, \mathrm{p}<0.05)$ than those without.

Conclusions: A small percentage (9\%) of prostate tumors from our RP series were located in the anterior zone. Most $(87 \%)$ were undetectable by DRE. A third of patients had positive family history. Despite the fact that $43 \%$ of APCa showed EPE, the incidence of $\mathrm{BF}(5 \%)$ and $\mathrm{CF}(2 \%)$ was overall low. All patients with $\mathrm{BF}$ had positive margins and significantly higher iPSA.

906 Clinical Validation of the 2005 ISUP Gleason Grading System in a Cohort of Intermediate and High Risk Men Undergoing Radical Prostatectomy

Sheila Faraj, Stephania M Bezerra, Kasra Yousefi, Helen Fedor, Stephanie Glavaris, Misop Han, Alan Partin, Elizabeth Humphrevs, Jeffrey Tosoian, Michael H Johnson, Elai Davicioni, Bruce Trock, Edward M Schaeffer, Ashley Ross, George J Netto. Johns Hopkins Medical Institution, Baltimore, MD; GenomeDx Biosciences, Vancouver, BC, Canada.

Background: To clinically validate the ISUP 2005 Gleason grading system for its ability to predict the occurrence of metastasis, biochemical recurrence, and prostate cancer specific mortality (PCSM) in a natural history cohort of intermediate and high risk men undergoing radical prostatectomy (RP).

Design: The primary endpoint of interest was defined as regional or distant metastases. We queried our institutional RP database for men with NCCN clinically localized intermediate to high risk disease undergoing RP between 1992 and 2010 with no additional treatment until the time of metastatic progression. 745 patients were identified and a case-cohort design was utilized to select $35 \%$ (265) men. Following the case-cohort design, 91 non-selected metastatic patients were added to the selected sub-cohort. Twenty-three patients had unavailable tumor blocks. Consequently, a total of 333 available RP samples were re-reviewed by two urologic pathologists, blinded to clinical outcome and Gleason score (GS) was reassigned per the 2005 ISUP modified Gleason system. Reassigned GS was compared to the original GS using survival c-index and decision curve analysis.

Results: Cumulative incidence of metastasis was $0 \%, 8.4 \%, 24.5 \%$ and $44.4 \%$ among specimens that were downgraded, unchanged, had one point GS increase and two point GS increase, respectively. The reassigned Gleason grades raised the hazard ratio for metastasis in GS 8 and 9 compared to GS 7 from 2.77 and 5.91 to 3.49 and 9.31, respectively. The survival concordance index of pathologic GS increased from 0.70 to 0.80 when samples were re-graded at 5 years post RP. The concordance index of the reassigned GS was higher than the original GS (C-index: 0.77 vs 0.64$)$ for predicting PCSM at 10 years post RP.
(C) Survival c-index at 10 Years Post-RP - PCSM

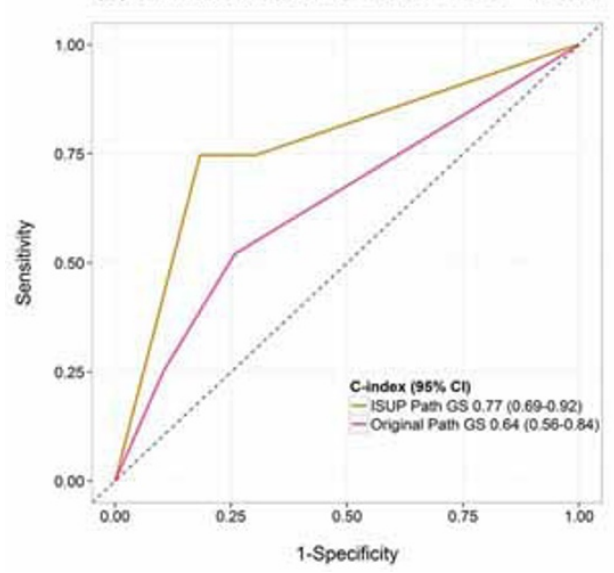

Conclusions: The ISUP 2005 Gleason grading system improved the prediction of metastasis and PCSM in clinicopathological models. This validates the updated Gleason grading system using an unambiguous clinical endpoint and highlights the need for reassignment of Gleason grading according to ISUP 2005 system when considering comparisons of novel biomarkers to clinicopathological variables in archival cohorts.

907 The Utility of HNF 1 Beta Immunohistochemical Staining in the Diagnosis of Malignant Germ Cell Tumors of the Testis

Faysal A Fedda, Charles Quick, Emily Holthoff, Neriman Gokden, Roni M Cox. UAMS, Little Rock, AR.

Background: Hepatocyte nuclear factor 1 beta (HNF 1 beta) is a transcription factor that plays a part in embryonal development and glucose homeostasis. Recent immunohistochemical (IHC) studies have shown a potential diagnostic use of HNF 1 beta in the differential diagnosis of ovarian neoplasms and in the differential diagnosis of chromophobe renal cell carcinoma. In a previous study, positive HNF 1 beta IHC staining was observed in yolk sac tumors of the ovary, but was negative in dysgerminomas. The goal of this study is to examine the immunohistochemical staining of HNF 1 beta in germ cell tumors of the testis and determine the potential usefulness of this stain as a diagnostic marker.

Design: IHC staining on whole sections of paraffin-embedded, formalin fixed tissue blocks was performed to include 16 cases of embryonal carcinoma, 15 cases of seminoma, 14 cases of yolk sac tumor, 7 cases of teratoma, and 3 cases of choriocarcinoma. Slides were stained with HNF 1 beta antibody (Sigma-Aldrich, rabbit polyclonal, 1:200 dilution) and evaluated with only nuclear staining considered positive. The HNF 1 beta slides were evaluated for staining and graded as follows: 0 - no staining, $1+-<10 \%, 2+-10-25 \%, 3+-25-49 \%$, and $4+-50 \%$ or greater. Cases with $3+$ and $4+$ staining were considered positive.

Results: The results of HNF1-beta IHC staining are summarized in the table below. Yolk sac tumors showed positive nuclear staining in $12 / 14$ cases (85.7\%). Teratoma showed positive nuclear staining in $5 / 7$ cases $(71.4 \%)$ with predominantly positive staining in the glandular elements and to a lesser extent in the squamous elements. Embryonal carcinoma was positive in 1/16 cases (6.3\%). Using a cut-off of $25 \%$ ( $3+$ and $4+)$ staining, no positive cases were observed in seminoma $(0 / 15)$ or choriocarcinoma $(0 / 3)$. Syncytiotrophoblasts were noted to have strong positive cytoplasmic staining. Sensitivity and specificity of HNF1-beta for yolk sac tumor was $86 \%$ and $85 \%$, respectively.

\begin{tabular}{|l|l|l|l|l|l|}
\hline Germ Cell Tumor & 0 & $1+$ & $2+$ & $3+$ & $4+$ \\
\hline Embryonal Carcinoma & 8 & 7 & 0 & 0 & 1 \\
\hline Yolk Sac Tumor & 0 & 1 & 1 & 3 & 9 \\
\hline Seminoma & 11 & 3 & 1 & 0 & 0 \\
\hline Teratoma & 0 & 2 & 0 & 3 & 2 \\
\hline Choriocarcinoma & 2 & 0 & 1 & 0 & 0 \\
\hline
\end{tabular}

Conclusions: HNF1-beta IHC staining may be a useful marker in the differential diagnosis of germ cell tumors of the testis. Yolk sac tumors tend to display strong, diffuse nuclear staining, as does the glandular elements of teratoma. Embryonal carcinomas, seminomas, and choriocarcinomas tend to display no staining or only weak nuclear staining in less than $25 \%$ of the neoplastic cells.

908 Ability to Accurately Diagnose Prostate Cancer Gleason Grade / Score Using Automated Image Analysis Tools with Multiplex Immunofluorescence

Gerardo Fernandez, Richard Scott, Faisal Khan, Michael J Donovan. Icahn School of Medicine at Mount Sinai Hospital, New York, NY.

Background: The Gleason score (GS) is the most widely used tissue-based architectural and morphological assessment of prostate cancer (PCa). Manual pathologist scoring of H\&E stained tissue sections is limited by intra and inter-observer reproducibility. We employed multiplex immunofluorescent (MIF) morphometry of PCa using gland rings to develop an automated $\mathrm{PCa}$ grading system and compared this approach with standard pathologist assessment. 
Design: 324 prostatectomy images from previously stained tissue arrays using an MIF assay of CK-18 (epithelial cells), CK 5/6 (basal cells), Androgen Receptor (AR), Ki67, AMACR and DAPI (nuclear marker) were analyzed. Images were captured with a Nikon i90 microscope equipped with a CRI multiplex camera. In addition to the clinical GS and dominant Gleason grade (GG), each image was reviewed by a pathologist to generate an image specific GG. We employed novel gland ring segmentation algorithms and nuclear architecture quantification to univariately and multivariately predict Gleason differentiation.

Results: The automated MIF Gleason correlated with both the image-specific GG and clinical Gleason GS; the best Pearson's correlation co-efficient was -0.48 and -0.27 respectively. In a multivariate model, we achieved an accuracy of $84 \%$ in predicting the image GG and $85 \%$ for the clinical GS. Dominant GG 3 accuracy was similar for both clinical and image derived GG (Spec $=92 \%$, NPV $=89 \%$ vs. Spec $=97 \%$, NPV $=$ $85 \%$ ). Dominant GG 4 was more accurate with the image GG (Sens $=62 \%$, PPV $=70 \%$ vs. Sens $=19 \%, \mathrm{PPV}=56 \%$ ). Additionally, the features correlated strongly with clinical progression (concordance index $(\mathrm{CI})=0.89$ ), significantly outperforming the clinical GS $(\mathrm{CI}=0.78)$.

Conclusions: Automated image analysis using MIF morphometry improves discrimination of dominant Gleason grade 4 vs. clinical Gleason. Such approaches should improve risk assessment and positively impact on post-treatment decisionmaking.

909 Warty Penile Intraepithelial Neoplasia: Morphological and Viral Characterization in 24 Cases Using Laser Capture Microdissection (LCM)PCR

Maria Jose Fernandez Nestosa, Nuria Guimera, Diego F Sanchez, Sofia Canete, Elsa F Velazquez, David Jenkins, Wim Quint, Antonio L Cubilla. Universidad Nacional de Asunción, Asunción, Paraguay; DDL Diagnostic Laboratory, Rijswijk, Netherlands; Instituto de Patología e Investigación, Asunción, Paraguay; Facultad de Ciencias Médicas-UNA, Asunción, Paraguay; Miraca Life Sciences and Tufts University, Boston, MA.

Background: Penile precancerous lesions are classified in non-HPV and HPV-related. Lesions related to the virus comprise variable morphologies, more frequently basaloid or mixed warty-basaloid. Pure warty PeIN are unusual lesions and they are typically part of multifocal PeIN.

Design: The objectives of the study were to morphologically characterize and to detect HPV genotypes in warty PeIN using Laser Capture Microdisection (LCM) technique. PCR analyses was performed by $\mathrm{SPF}_{10}$-DEIA-LiPA $_{25}$ (version 1). DNA quality of negative cases was confirmed by RNAseP/PhHV qPCR

Results: Warty PeINs clinically presented as part of a multicentric lesion in the majority of the cases. They were associated with other subtypes of PeIN in the same specimen. Grossly they were sharply delineated slightly elevated reddish granular or villous lesion. Microscopically there were parakeratosis, papillomatosis, pleomorphic koilocytosis, squamous cell maturation and atypias involving full epithelial thickness. Clear cells were prominent. High risk HPV genotypes were identified in 20 cases and low risk HPVs, in 4 cases. Each lesion demonstrated a single HPV genotype. Among the high risk group, HPV 16 and 56 were the commonest present in 5 cases each, followed by HPV 39, in 3 cases. Other high risk genotypes were 18, 30, 33, 66 and 73. Low risk HPVs detected were 11 (2 cases), 84 and 87.

Conclusions: Warty PeIN was pathologically distinctive, HPV-related, usually multicentric and, unlike other HPV induced subtypes of PeIN where HPV 16 predominates, it showed an heterogeneous spectrum of several HPV genotypes.

910 Assessing \% Gleason Pattern (GP) 4 in Needle Biopsies: An Interobserver Reproducibility Study

SW Fine, J Sarungbam, HA Al-Ahmadie, Y-B Chen, A Gopalan, SJ Sirintrapun, VE Reuter, SK Tickoo. Memorial Sloan Kettering Cancer Center, New York, NY.

Background: Gleason grading on prostatic needle biopsy is a key factor in determining eligibility for and progression on active surveillance (AS). While Gleason score (GS) $\leq$ 6 is the most common GS in AS-eligible patients, it has been suggested that GS $3+4=7$ on needle biopsy may also be managed by AS, when the volume of Gleason pattern 4 (\%GP4) is low. The reproducibility of assessing \%GP4 has not been well studied. Given its potential impact on management with AS, this study sought to determine the interobserver reproducibility for \%GP4 among a group of urologic pathologists. Design: Six urologic pathologists assessed the \%GP4 in each of 49 digitally scanned (Aperio CS) prostatic needle biopsy cores which were reported clinically as GS 3+4=7. For statistical analysis, $\% \mathrm{GP} 4$ was grouped as $\leq 10 \%, 11-50 \%$ and $>50 \%$ to better identify agreement in cases with low volume GP 4 .

Results: Overall agreement among the 6 pathologists using \%GP4 groups was moderate $(\kappa=0.42)$. There was agreement for \%GP4 group among all observers in $15 / 49(31 \%)$ cases; however, agreement was seen in $5 / 6$ observers in $30 / 49(61 \%)$ cases and in $4 / 6$ observers in $42 / 49(86 \%)$ cases. 10 cases had at least one observer who assigned $>50 \%$ GP4 (i.e. GS $4+3=7) ; 8(80 \%)$ of these had at least $5 / 6$ observers who assigned $\geq 30 \%$ GP4. 9 cases had at least one observer assigning $0 \%$ GP4 (i.e. GS $3+3=6) ; 7(78 \%)$ of these had at least $5 / 6$ observers who assigned $\leq 10 \%$ GP 4 .

Conclusions: 1 . While the overall concordance for determining \%GP4 among all pathologists in this study is only moderate, most pathologists concurred on the assignment of \%GP4 grouping in the overwhelming majority of cases.

2. For cases with low volumes of Gleason pattern 4 , while differences in interobserver interpretation exist, the differences appear to be minor, and may not significantly alter management in centers in which GS $3+4=7$ with low $\% \mathrm{GP} 4$ is considered eligible for active surveillance.
3. For cases with high volumes of Gleason pattern 4 , interobserver interpretational differences also seem to be too modest to affect clinical management $(4+3$ versus $3+4$ with high \%GP4).

4. Achieving reasonable and potentially clinically useful levels of interobserver reproducibility likely requires grouping \%GP4 into clinically/prognostically meaningful categories; a larger cohort study is underway to help determine ideal groupings.

911 A Comparison of Multiparametric Magnetic Resonance Imaging of the Prostate and Histologic Prostatectomy Findings - A Single Institution's Experience

Erin Faber, Julie Anne Bishop, Mostafa M Fraig, Albert Seow, Houda Alatassi. University of Louisville, Louisville, KY

Background: Pretreatment staging of prostate cancer can help in determining patients' eligibility for surgical resection. Detection of prostate cancer by multiparametric magnetic resonance imaging (mpMRI) has shown consistently high specificity and invariably high negative predictive values. Although extracapsular extension and seminal vesicle involvement are more of a staging issue rather than one of detection, the findings from these two areas are nevertheless very important in treatment planning. Design: We queried our institution's database to identify radical prostatectomy specimens with a preoperative mpMRI using 2 tesla scanner and revealed 21 cases. All of the mpMRI scans were examined by a single radiologist. Based on the latest AJCC staging, 11 cases had T2 disease, 9 cases had T3 and one case had T4 disease. In all cases the entire prostatic capsule was submitted for histopathological evaluation. The prostatectomy specimens were reviewed for extraprostatic extension (EPE) and seminal vesicle involvement (SVI) by a single pathologist who is experienced in prostate pathology.

Results: For SVI, there was $100 \%$ agreement between the mpMRI results and the histopathological evaluation in all 21 cases. In regards to EPE, false negative results were identified in three cases. In addition, one case was reported as suspicious on MRI, however, no EPE was identified by histopathologic evaluation. In all cases the EPE was focally present in less than one high power field and only in one section.

Conclusions: Our study supports the utility of mpMRI in the detection and staging of prostate cancer. As a non-invasive technique, mpMRI carries less morbidity and risk for patients. This imaging has the potential to markedly improve the choice of treatment. MRI is very sensitive and specific for detecting SVI and very specific for EPE-both of which have a significant clinical impact.

912 BK Virus: Unveiling a Pathogen in 46 Autopsies of Allogeneic Stem Cell Transplant Recipients

Kenneth Friedman, Rima Pai, William F Glass, Elizabeth Shpall, Katy Rezvani, Ala Abudayyeh, Miao Zhang. University of Texas MD Anderson Cancer Center, Houston, TX; University of Texas Health Science Center at Houston, Houston, TX.

Background: BK virus (BKV) is known to be causally related to chronic kidney disease $(\mathrm{CKD})$ in renal transplant recipients. Seropositive BK in adults ranges from $60-80 \%$; however in immunocompromised patients, BK viruria can progress to BK viremia and lead to tubulo-interstitial inflammation known as BK virus nephropathy. In allogeneic hematopoietic stem cell transplant (HSCT) recipients, BKV is associated with hemorrhagic cystitis. However, the relationship between BKV and CKD in HSCT recipients is not as well established and deserves investigation, as up to $65 \%$ of HSCT patients develop CKD. We focused on investigating the prevalence of BKV in autopsy kidneys from patients that had undergone allogeneic HSCT.

Design: The electronic medical record was searched retrospectively for patients who had undergone an allogeneic HSCT and had an autopsy performed between 2004 and 2012. Formalin fixed paraffin embedded kidney tissue blocks from these patients were analyzed by light microscopy and anti-SV40 immunohistochemistry for detection of BKV. Cases were considered positive for BKV if moderate-to-strong staining was present in nuclei of renal tubular epithelial cells with appropriate controls and semiquantitated based on percentage of total tubular epithelial cells affected $(<25 \%$ vs. $>25 \%$ ). Additional clinicopathologic parameters were collected.

Results: A total of 46 patients were included in the study. All patients (100\%) had positive SV40 staining in at least $25 \%$ of tubular epithelial cells with 16 patients $(35 \%)$ having more than $25 \%$ involved tubular cells. Of thirteen patients who had undergone pre-mortem BKV testing, $10(77 \%)$ tested positive. Light microscopic findings at autopsy included unremarkable renal parenchyma in $25(54 \%)$ cases, hemorrhagic cystitis in 8 $(17 \%)$ cases and viral cytopathic effect in the tubules in 1 case $(2 \%)$

Conclusions: BK virus is highly prevalent in kidneys at the time of death in patients who had a history of allogeneic HSCT. Although there are many factors involved in chronic kidney disease in HSCT patients, the finding of universal presence of BK virus in autopsy kidneys warrants rigorous study.

913 Immunohistochemical Comparison of BAP1 Immunoexpression Status between Needle Biopsies and Nephrectomies in Clear Cell Renal Cell Carcinoma

Paul Friedman, Farrah Homayoun, Vitaly Margulis, Jeffrey Cadeddu, Dinesh Rakheja, James Brugarolas, Payal Kapur. University of Texas Southwestern Medical Center, Dallas, TX

Background: We have previously shown that BAP1 immunohistochemistry (IHC) in clear cell renal cell carcinoma (ccRCC) nephrectomy specimens has outstanding correlation with $B A P 1$ mutation status and that loss of BAP1 is associated with reduced rate of ccRCC-specific survival (HR, 3.06; 95\% CI, $2.28-4.10 ; \mathrm{p}=6 \cdot 10^{-14}$ ).

Design: We retrospectively evaluated 357 patients that underwent core biopsies of renal masses from January 2008 to July 2015. Nephrectomy was performed in 124 $(35 \%)$ cases, with $83(67 \%)$ diagnosed as ccRCC. Adequate tissue was available for 53 
paired core biopsy/nephrectomy cases. Additionally, 19 post-nephrectomy metastatic ccRCC cases with core biopsies of metastatic site were reviewed. BAP1 IHC expression between core biopsies and representative section from nephrectomies was evaluated to determine concordance rates

Results: Of the 53 core biopsies, $49(92 \%)$ were BAP1 positive, and $4(8 \%)$ were negative. Renal biopsies correctly identified BAP1 status in $44(90 \%)$ of 49 BAP1positive and all $4(100 \%)$ BAP1-negative cases. In the 5 discordant cases with positive BAP1 staining in the core, the representative nephrectomy section showed only subclonal loss of BAP1 (focally negative). Overall concordance in this cohort was $48 / 53(91 \%)$. In the metastatic ccRCC cohort, correlation between nephrectomy and metastatic lesion biopsy was $18 / 19(95 \%)$. Seven (37\%) of the 19 cores from metastatic ccRCC were BAP1 negative, and all showed BAP1 loss in the nephrectomy ( 3 with only focal loss in the higher grade area). One nephrectomy with focal loss had a metastatic biopsy that was positive.

\begin{tabular}{|l|l|l|}
\hline Table 1. BAP1 status biopsy vs nephrectomy \\
\hline \multirow{2}{*}{ Nephrectomy BAP1 status } & \multicolumn{2}{|l|}{ Biopsy BAP1 status } \\
\cline { 2 - 3 } & Pos (49) & Neg (4) \\
\hline Positive (44) & 44 & 0 \\
\hline Negative (4) & 0 & 4 \\
\hline Focal negative (5) & 5 & 0 \\
\hline
\end{tabular}

\begin{tabular}{|l|l|l|}
\hline Table 2. BAP1 status nephrectomy vs metastatic lesion biopsy \\
\hline \multirow{2}{*}{ Nephrectomy BAP1 status } & \multicolumn{3}{|l|}{ Biopsy BAP1 status } \\
\cline { 2 - 3 } & Pos (12) & Neg (7) \\
\hline Positive (11) & 11 & 0 \\
\hline Negative (4) & 0 & 4 \\
\hline Focal negative (4) & 1 & 3 \\
\hline
\end{tabular}

Conclusions: Biopsy of renal mass yields adequate material for assessing BAP1 status by IHC, and can serve as an important prognostic indicator that can inform preoperative risk stratification and guide treatment planning. Instances with focal BAP1 loss in nephrectomies demonstrate an aggressive subclone with greater propensity for metastasis.

\section{EZH2 as a Novel Target for Aggressive Clear Cell Renal Cell}

\section{Carcinoma}

Paul Friedman, Alana Christie, Xian-Jin Xie, Dinesh Rakheja, James Brugarolas, Payal Kapur. University of Texas Southwestern Medical Center, Dallas, TX.

Background: Recently overexpression and amplification of EZH2 has been described in tumors including carcinomas, lymphomas, and sarcomas. We investigate the clinicopathologic significance of EZH2 using immunohistochemistry (IHC) in a cohort of 145 cases for which we have VHL, BAPI and PBRM1 sequencing data and extensive clinical annotation.

Design: IHC for EZH2 was performed on sections of tissue microarrays (TMAs) constructed from 145 primary ccRCC cases (stages I to II) treated at our institution with nephrectomy. Duplicate $1.0 \mathrm{~mm}$ cores of representative tumor were obtained from each case to construct the TMAs. Based on published literature, a cut-of value of $\geq 25 \%$ nuclear EZH2 expression in the tumor cells was considered as overexpression. EZH2 overexpression was correlated with pathological parameters using t-test (for continuous variables) and Fisher's exact test (for categorical variables).

Results: Benign kidney and low-grade ccRCC were predominantly negative for EZH2. EZH2 overexpression was seen in $11 \%$ of the cohort. Interestingly, EZH2 is located on chromosome $7 \mathrm{q} 35$, a region amplified in $13 \%$ of ccRCC. Our preliminary analyses showed that tumors with EZH2 overexpression were associated with aggressive features including higher nuclear grade, sarcomatoid and rhabdoid histology, presence of necrosis, and higher TNM and pathologic T stage ( $p<0.05$ for all). Most strikingly, the median overall survival for patients with EZH2 overexpressing tumors was only 1.1 years $(95 \% \mathrm{CI}, 0.8-1.4)$ compared to 11.4 years $(95 \% \mathrm{CI}, 10.9-11.9)$ for patients with tumors showing low EZH2 expression, corresponding to $\mathrm{HR}$ of 7.4 (95\% CI, 3.2-17.2, Figure). RNA-seq information obtained from the KIRC-TCGA Data Portal showed similar results (https://tcga-data.nci.nih.gov/tcga/).

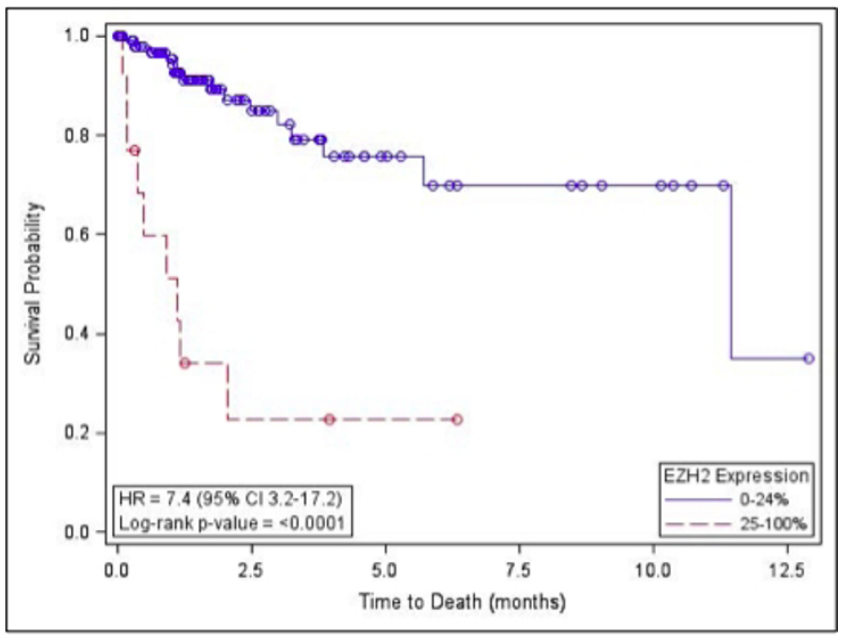

Conclusions: Our study indicates that ccRCCs with EZH2 overexpression are associated with aggressive pathologic features and poor clinical outcomes. EZH2 IHC can be easily incorporated into practice to identify this subset of ccRCC that may benefit from upfront aggressive or targeted treatment options.

915 Proliferation Index of Different Gleason Grade 4 Prostatic Adenocarcinoma Morphologies

Liying Fu, Michael Hwang, Adebowale Adeniran, Peter A Humphrey. Yale University School of Medicine, New Haven, CT.

Background: Gleason score is one of the most powerful prognostic indicators for prostatic adenocarcinoma, and its accurate assessment is important in determining patient management and therapy. Gleason grade 4 prostatic cancer is a morphologically heterogeneous group, and includes cribriform glands, poorly-formed glands, and fused glands. Recent data indicate that cribriform morphology has a more aggressive clinical course. Studies have shown that higher Gleason score and more clinically aggressive prostate cancers tend to have a higher proliferation index as assessed by Ki67 labeling index (Ki67 LI). However, there are no data on the proliferation index of different Gleason grade 4 morphologies. Our study aimed to compare Ki67 LI among different Gleason grade 4 morphologies.

Design: The pathology database was searched for prostatectomy specimens with a diagnosis of prostatic carcinoma Gleason score 7 to 9 from 2009 to 2013. The 2011 AFIP modified Gleason scheme was used in assigning Gleason scores. All available cases were reviewed by two genitourinary pathologists and areas with different Gleason patterns were circled. All selected blocks were divided into five groups based on the Gleason pattern: Gleason 3 (G3), Gleason 4 with cribriform (G4-C), Gleason 4 with poorly formed glands (G4-P), Gleason 4 with fusion (G4-F), and Gleason 5 (G5). Ki67 immunohistochemical stains were performed, and the Ki67 LI was defined as the number of immunoreactive cells divided by the total counted cells on printed color images taken from areas with highest Ki67 positivity. All data are presented as mean \pm SE, and non-paired $t$ test or Brown-Forsythe test follow by Games-Howell post hoc test were used for statistical analysis.

Results: 145 blocks were selected from 62 cases and were stained for Ki67. The Ki67 LI for prostatic adenocarcinoma with different Gleason patterns is listed in Table 1.

\begin{tabular}{|l|l|l|l|l|l|}
\hline Gleason pattern & G3 & G4-C & G4-F & G4-P & G5 \\
\hline Ki67 LI (\%) & $2.99 \pm 0.24$ & $5.78 \pm 0.54$ & $3.90 \pm 0.31$ & $3.86 \pm 0.33$ & $12.93 \pm 3.64$ \\
\hline Foci number (n) & 79 & 58 & 44 & 53 & 8 \\
\hline
\end{tabular}

Among groups with Gleason grade 4 morphologies, G4-C showed a higher Ki67 LI than G4-F $(\mathrm{p}<0.01)$ and G4-P $(\mathrm{p}<0.01)$. G4-P showed a small but statistically significant higher Ki67 LI than G3 $(\mathrm{p}<0.05)$. G5 showed a higher Ki67 LI compared to any grade 4 patterns.

Conclusions: Gleason grade 4 prostate cancer with cribriform growth demonstrates a higher Ki67 LI than other Gleason grade 4 morphologies, consistent with the more clinically aggressive nature of cribriform adenocarcinoma of the prostate.

916 The Prognostic Significance of Perineural Invasion in Prostatic Core Biopsies

Manal Gabril, Tianzhu Zhang, Jonathan Izawa, Madeleine Moussa. Western University/ London Health Sciences Centre, London, ON, Canada.

Background: The prognostic significance of perineural invasion (PNI) in prostate cancer (Pca) is debated. PNI is defined as the presence of cancer tracking along or around a nerve. Since PNI is a major mechanism of Pca extension from parenchyma to periprostatic soft tissue, PNI which is sampled on prostate core biopsy may signal an increased likelihood of extraprostatic extension (EPE) of cancer in radical prostatectomy (RP). The objective of the study is to evaluate the significance of PNI in the prostate core biopsy in predicting EPE/high pathological stage (specifically pT3a) in RP. Design: 389 prostatic core biopsies and their corresponding RP specimens performed at the LHSC were included (2010 to 2013). The cases were divided into "group 1" and "group 2": 188 and 201 cases with negative and positive PNI biopsy respectively. Clinicopathological data (age, PSA level, Gleason score (GS), \% of tumor volume, number of positive specimens in biopsy, presence or absence of EPE (focal/non-focal) and PNI) was collected.

Results: 65/188 (34.5\%) cases from "group 1" and 136/201 (67.6\%) cases from "group 2" showed positive EPE in the corresponding RP. 106/136 (79.7\%) cases in "group 2" had non-focal EPE ( $p=<0.001)$. PNI was significantly associated with EPE (Table 1). Furthermore, the cases were categorized into 4 groups: PNI - /EPE - (n=123), PNI - / $\mathrm{EPE}+(\mathrm{n}=65), \mathrm{PNI}+/ \mathrm{EPE}-(\mathrm{n}=65)$ and $\mathrm{PNI}+/ \mathrm{EPE}+(\mathrm{n}=136)$. There was a significant difference in Gleason Score, tumor volume and number of positive specimens between the 4 groups $(\mathrm{p}<0.001)$.

Table 1: Logistic regression analysis of PNI in biopsies for predicting EPE in RP

\begin{tabular}{|l|l|l|l|l|l|l|}
\hline \multicolumn{3}{|c}{ Univariate } & \multicolumn{5}{c|}{ Multivariate } \\
\hline & Odds Ratio & $95 \% \mathrm{CI}$ & $p$-value* & Odds Ratio & $95 \% \mathrm{CI}$ & $p$-value* \\
\hline EPE & 3.96 & $2.60-6.03$ & $<0.001$ & 2.34 & $1.38-3.98$ & $<0.001$ \\
\hline
\end{tabular}

${ }^{*} \mathrm{p}<0.05$ significant

Conclusions: The presence of perineural invasion in prostate core biopsy is significantly associated with EPE in RP which indicates high pathological stage (pT3a). Based on these results, perineural invasion in prostate core biopsies should be considered as an independent prognostic factor in selecting the management options (surgery vs. surveillance) for the patients. 
917 The Spectrum of Oncocytic Renal Tumors from Oncocytoma to Eosinophilic Variant Chromophobe Renal Cell Carcinoma - Is There a Need to Sub-Classify and What Is the Malignant Potential?

Ramya Gadde, Ravi Barod, Craig G Rogers, Nilesh S Gupta, Sean R Williamson. Henry Ford Health System, Detroit, MI.

Background: Oncocytoma is well-recognized as a benign renal neoplasm, and chromophobe renal cell carcinoma (RCC), whether classic or eosinophilic variant, is generally regarded as having less aggressive behavior than other RCC subtypes. We analyzed oncologic outcomes in our patients with such renal tumors, including oncocytoma, eosinophilic variant chromophobe RCC, classic chromophobe RCC, and tumors with oncocytic morphology that evaded definitive classification.

Design: Our pathology database was queried for oncocytomas, chromophobe RCCs, and other oncocytic renal neoplasms (unclassified, hybrid, and borderline tumors) diagnosed between 2006 and 2013. Other tumor subtypes, such as clear cell or papillary RCC with variant oncocytic morphology, were excluded

Results: A total of 126 cases were retrieved, including 38 oncocytomas, 31 eosinophilic variant chromophobe RCCs, and 14 oncocytic tumors with inconclusive morphology. These were compared to 43 chromophobe RCC with classic (non-eosinophilic) histology. Follow-up for 83 patients with oncocytic renal tumors, ranged from 1 month to 102 months (median 44), and revealed no adverse events (recurrence or metastasis), including 14 oncocytic tumors that were difficult to definitively classify.

\begin{tabular}{|l|l|l|l|l|l|l|l|l|l|}
\hline & \multicolumn{2}{|c|}{} & $\begin{array}{l}\text { Size } \\
\text { (me- } \\
\text { dian) }\end{array}$ & $\begin{array}{l}\text { Follow } \\
\text { up } \\
\text { months } \\
\text { (me- } \\
\text { dian) }\end{array}$ & $\begin{array}{l}\text { Stage } \\
\text { pT3a }\end{array}$ & $\begin{array}{l}\text { Recur- } \\
\text { rence }\end{array}$ & $\begin{array}{l}\text { Metas- } \\
\text { tasis }\end{array}$ \\
\cline { 2 - 10 } & $\leq 4 \mathrm{~cm}$ & $\begin{array}{l}>4- \\
7 \mathrm{~cm}\end{array}$ & $>7 \mathrm{~cm}$ & $\begin{array}{l}\text { Not } \\
\text { avail- } \\
\text { able }\end{array}$ & & & & & \\
\hline $\begin{array}{l}\text { Onco- } \\
\text { cytoma } \\
\text { (38) }\end{array}$ & 24 & 8 & 5 & 1 & 3.0 & 39.5 & $\begin{array}{c}\text { Not ap- } \\
\text { plicable }\end{array}$ & No & No \\
\hline $\begin{array}{l}\text { Eo- } \\
\text { sinophilic } \\
\text { chromo- } \\
\text { phobe } \\
\text { (31) }\end{array}$ & 23 & 4 & 4 & 0 & 2.85 & 48 & None & No & No \\
\hline $\begin{array}{l}\text { Border- } \\
\text { line (14) }\end{array}$ & 10 & 4 & 0 & 0 & 3.35 & 37.5 & 1 & No & No \\
\hline
\end{tabular}

As a control group, classic chromophobe RCCs were variably larger $(0.8$ to $23.0 \mathrm{~cm}$, mean $4.9 \mathrm{~cm})$, with a subset $(10 / 43)$ being pT3a, due to vein invasion (1), renal sinus and perinephric fat invasion (1), and perinephric fat invasion (8).

Conclusions: Our findings support the benign behavior of oncocytoma and nonaggressive behavior of eosinophilic variant chromophobe RCC and related borderline oncocytic neoplasms. Despite that some tumors exhibit oncocytic morphology that evade definitive classification, outcomes in these patients are generally good, with no disease recurrence or metastasis in this study. Taking into account the indolent behavior of these tumors may aid in designing clinical follow-up strategies and patient counseling, even when features are not perfect for diagnosis of oncocytoma.

918 Pathologic Findings in Patients with Targeted Magnetic Resonance Imaging-Guided Prostate Needle Core Biopsies Following Prior Negative Transrectal Ultrasound-Guided Prostate Needle Core Biopsies

Rachel Geller, Zachary J Dureau, M Andrew Toussaint, Sherif G Nour, Adeboye O Osunkoya. Emory University School of Medicine, Atlanta, GA.

Background: The likelihood of identifying prostate cancer $(\mathrm{PCa})$ decreases after the first negative transrectal ultrasound-guided biopsy (TRUS-GB), and after each subsequent negative TRUS-GB. In contrast to the systematic (but non-targeted) sampling approach of TRUS-GB, targeted magnetic resonance imaging-guided biopsies (TMRI-GB) target regions of the prostate suspicious for $\mathrm{PCa}$, based on findings on multiparametric MRI. There are limited studies in the literature examining the pathologic findings in patients with at least 1 prior negative TRUS-GB and subsequent TMRI-GB.

Design: A search was made through our Urologic Pathology files for prostate needle core biopsies that were obtained via TMRI-GB following at least one prior negative TRUS-GB from 2013-2015.

Results: Twenty seven cases were identified. Mean patient (PT) age was 63 yrs (range: 45 - $78 \mathrm{yrs}$ ). Ten of 27 (37\%) PTs had 1 prior benign TRUS-GB, 17/27 (63\%) had 2 or more prior benign TRUS-GBs prior to TMRI-GB. At the time of TMRI-GB, all PTs were described as having at least 1 focal abnormality suspicious for PCa on diagnostic MRI An average of 5 lesions were identified per PT, and an average of 16 cores (range: 9-24) were collected per TMRI-GB procedure. Overall, TMRI-GB results were malignant in $9 / 27(33 \%)$ patients. TMRI-GB findings were malignant in 4 of $10(40 \%)$ patients with 1 prior benign TRUS-GB and 5 of 17 (29\%) with 2 prior benign TRUS-GBs. The Gleason scores (GS) and Grade Groups in these PTs were as follows: 3/9 (33\%) PTs had a GS of $3+3=6$ (Grade Group 1), 3/9 (33\%) PTs had a GS of 3+4= 7 (Grade Group 2), $2 / 9$ PTs $(22 \%)$ had a GS of $4+4=8$ (Grade Group 4$)$, and 1/9 PTs had a GS of 5+4=9 (Grade Group 5). Non-cancerous findings included: unremarkable, benign prostatic tissue (54\%), inflammation (40\%), high grade prostatic intraepithelial neoplasia (3\%), benign fibromuscular tissue $(2 \%)$, and benign prostatic hyperplasia $(1 \%)$.

Conclusions: In our study, TMRI-GB identified PCa in 33\% of patients with at least one prior negative TRUS-GB, in contrast to the $10-17 \%$ detection rate of $\mathrm{PCa}$ on repeat TRUS-GB of PTs with one prior negative TRUS-GB documented in the literature. TMRI-GB appears to be a more effective alternative to repeat TRUS-GB in patients with at least one prior negative TRUS-GB.
919 HER2 Status in Metastazising Urothelial Bladder Cancers Vera Genitsch, Philipp Niederberger, Aurel Perren, Roland Seiler, George N Thalmann, Achim Fleischmann. University of Bern, Bern, Switzerland.

Background: Approximately $10 \%$ of invasive urothelial cancers of the bladder (UBC) are Her2 amplified and might be candidates for anti-Her2 targeted therapies. Better knowledge about the distribution of these targets in primary tumors and metastases is necessary to assess the potential benefit of these therapies.

Design: Out of 152 patients with UBC treated by cystectomy and pelvic lymphadenectomy 31 had one and 121 had $\geq$ two lymph node metastases. Tissue microarrays (TMA) were constructed containing two samples each from the primary tumors and from corresponding lymph node metastases $(n=1-4)$. Her2 gene status was determined by fluorescence in-situ hybridization using the categories non-amplified, borderline-amplified and amplified (HER2/CEP17 fluorescence ratios <1.8, 1.8-2.2 and $\geq 2.2$ ).

Results: Her2 status in the tumor tissues with informative results from the two TMA spots: (a) of 116 primary UBC $88 \%$ were homogeneously non-amplified, $8 \%$ were homogeneously amplified and in $4 \%$ one spot was amplified, the other was not; (b) corresponding data from the 214 lymph node metastases were $77 \%, 20 \%$ and $3 \%$.

An amplification status could be assigned to the primary tumor and multiple corresponding metastases in 86 patients: (a) $88 \%$ of these patients had non-amplified primary UBC, in $73 \%$ all metastases were non-amplified, in $2 \%$ all were amplified and in $13 \%$ there were amplified and non-amplified metastases; (b) the remaining $12 \%$ of these patients had amplified primary UBC, in $7 \%$ all metastases were amplified and in $5 \%$ there were amplified and non-amplified metastases.

Conclusions: Our data suggest that only a moderate fraction of metastases from Her2 amplified primary UBC is targetable by anti-Her2 therapies. However, the metastatic component of a subset of non-amplified primary UBC reveals de novo Her2 amplification. Therefore, determination of Her2 status in the metastases is useful.

\section{Testicular Torsion: A Single Tertiary Care Institution Study of 210 Cases}

Alireza Ghaffarieh, Karen Elizabeth Trevino, Thomas MUlbright, Muhammad TIdrees. Indiana University School of Medicine, Indianapolis, IN.

Background: Testicular torsion is a surgical emergency; accurate diagnosis and prompt treatment may help salvage testicular function. Various radiological and laboratory diagnostic modalities are used to diagnose this condition and treat in a timely fashion. Unfortunately orchiectomy is the outcome in many cases, and these end up on pathologists' desks for evaluation. Accurate diagnosis is important for various reasons, including litigation and patient reassurance. We evaluated orchiectomies performed for testicular torsion at our institution and analyzed clinical and morphologic features. Design: An electronic data search of our institutional files was performed for consecutive surgical cases diagnosed as testicular torsion from 2001-2014. The surgical pathology reports were evaluated for morphologic features. Clinical data was obtained from clinical notes. All consultation cases were excluded.

Results: Two hundred and ten consecutive orchiectomies were evaluated. 28 were adults with a mean age of 34.4 years (range 19-58). The remaining 182 were children ( $>1$ yr. 92, 1-12 months 72 and $<1$ month 18). 142 (67\%) were white while $39(18.6 \%)$ were African American. Ethnicity was not available or other in the remaining. These ethnicities are similar to those of the served population. The left testis was involved more often than right ( $63 \%$ vs $37 \%)$. The time of year of presentation was evaluated to assess for seasonal variation. There was no statistical difference among different months and seasons of the year. Symptoms varied in adults and children; adults most commonly presented with sudden scrotal pain and swelling while children presented with a firm mass and abnormal position of the testis. The most common microscopic finding was hemorrhage (84\%) with associated infarction of the parenchyma (83\%). Other findings included combinations of venous congestion, edema, hemosiderin deposition, fibrosis and calcifications. The latter two were almost always found in patients who presented with gradual pain and delayed treatment.

Conclusions: The most vulnerable age for developing torsion is before age 1 year, when almost $50 \%$ of childhood cases present ( $43 \%$ overall). Torsion in adults occurs mostly in young men, probably indicating a developmental predisposition in conjunction with an active lifestyle as risk factors. Left sided torsion is more common. There is no apparent ethnic predilection or evidence of seasonal variation. The sign and symptoms for adults and children are different. Morphological changes correspond to the chronicity of the torsion.

\section{Testicular Appendageal Tumors Evaluated in Orchiectomies, a} Single Institution Experience

Alireza Ghaffarieh, Erin Baumgartner, Thomas M Ulbright, Muhammad T Idrees. Indiana University School of Medicine, Indianapolis, IN; University of Louisville, Louisville, KY.

Background: Tumors arising in the testicular appendages, such as the epididymis, rete testis, and other hilar structures, are rare and consequently have primarily been reported only in small series and case reports. The rarity of these tumors poses diagnostic challenges for the general pathologists, unfamiliar with many of these entities. A comprehensive audit of neoplasms of the testicular appendages is not available in the literature. We documented numerous rare entities specific to these structures in order to provide a clear idea of what may arise in association with these structures.

Design: We electronically retrospectively searched for cases occurring from 19812015. We reviewed 5392 cases, and identified a total of 57 cases fulfilling the search parameters. All reports were evaluated, and clinical information was obtained from clinician notes. 
Results: A wide morphologic spectrum of epithelial and nonepithelial neoplasms was identified in relation to the appendageal structures. The most common benign neoplasm was adenomatoid tumor (10), followed by serous cystadenoma of the epididymis and benign epididymal cyst ( 8 each). The most common malignant neoplasm was malignant mesothelioma (7). Four of 7 mesotheliomas were epithelioid while the remaining was of papillary type. For details see table 1 .

\begin{tabular}{|l|l|l|l|}
\hline Diagnosis & Average Age & Age Range & \# of Cases \\
\hline Adenomatoid tumor & 43 & $31-65$ & 10 \\
\hline Benign epididymal cyst & 50 & $21-80$ & 8 \\
\hline Serous cystadenoma & 42 & $22-95$ & 8 \\
\hline Mesothelioma & 63 & $47-85$ & 7 \\
\hline Rete testis carcinoma & 59 & $32-74$ & 5 \\
\hline Sertoliform cystadenoma & 53 & $21-84$ & 4 \\
\hline Serous carcinoma & 33 & $30-36$ & 3 \\
\hline Benign cyst of tunica albuginea & 65 & $61-72$ & 3 \\
\hline Mesothelial hyperplasia & 50 & $24-75$ & 3 \\
\hline Mullerian type cyst & 17 & $16-19$ & 2 \\
\hline Retinal anlage tumor & 1 & 1 & 2 \\
\hline Clear-cell adenocarcinoma & 70 & 70 & 1 \\
\hline Cystadenocarcinoma of epididymis & 64 & 64 & 1 \\
\hline
\end{tabular}

Conclusions: The neoplasms and nonneoplastic lesions arising in association with testicular appendages display a broad spectrum of morphologies and occur over a broad age range. It is important to accurately diagnose these tumors and differentiate them from tumors arising from the testis, especially germ cells and metastasis from other organs.

922 Occult Metastases in Pelvic Lymph Nodes Removed during Radical Cystectomy for Urothelial Carcinoma

Briana R Gibson, James L Ellenburg, Todd M Stevens, William E Grizzle, Soroush RaisBahrami, Jennifer B Gordetsky. University of Alabama at Birmingham, Birmingham, AL.

Background: In patients with high-grade, muscle-invasive urothelial carcinoma (UC) of the bladder, pelvic lymphadenectomy during radical cystectomy demonstrates a wellestablished survival advantage, increasing with the number of lymph nodes removed, even if negative for metastatic disease. This finding may potentially be explained by the presence of occult micrometastases and isolated tumor cells (ITCs) within lymph nodes reported as negative by routine histologic evaluation.

Design: We queried archives from 2000 to 2014 for radical cystectomy specimens with high-grade UC invading the perivesical tissue and negative lymph nodes (pT3N0). Five levels with interval spacing of 50 microns between each were cut for each lymph node block. Two sections were cut per level: one stained for hematoxylin and eosin and the second for AE1/AE3. Micrometastases were defined as tumor deposits $>0.2 \mathrm{~mm}$ but $<2$ $\mathrm{mm}$. ITCs were defined as $\leq 0.2 \mathrm{~mm}$. Medical records and publically available Social Security Database were reviewed to assess patient clinical and survival outcomes. Results: We identified 21 cases with a total of 370 lymph nodes. 6/21 (29\%) patients had occult metastases on prior routine histologic evaluation. 5 patients had metastatic UC, for a total of 8 positive lymph nodes. One patient had metastatic prostatic adenocarcinoma. Three patients had micrometastases and 3 patients had ITCs. Micrometastases and ITCs were each present in $4 / 8$ positive lymph nodes. 2 of the $6(33 \%)$ patients had lymphovascular invasion identified in the primary tumor. There was no significant difference in cancer-specific survival and overall survival between the group of patients who remained N0 vs those upstaged due to discovery of micrometastases and ITCs on Kaplan-Meier survival curve analysis: log-rank test p-value $=0.46$ and 0.42 , respectively.

Conclusions: In patients with pT3 UC undergoing cystectomy, we demonstrated the presence of occult metastases in $29 \%$ of patients. ITCs and micrometastases have rarely been studied in UC, where removal of "negative" lymph nodes confers a survival advantage. The high percentage of occult metastases present in these cases may explain the proven survival advantage of removing previously considered "negative" lymph nodes. This finding might also have implications in the histologic evaluation of lymph nodes.

923 Urothelial Carcinoma of the Upper Urinary Tract: A Clinicopathologic Study of 100 Cases

Syed Gilani, Bogdan A Czerniak, Charles C Guo. MD Anderson Cancer Center, Houston, TX.

Background: Urothelial carcinoma of the upper urinary tract (UC-UUT) is an uncommon disease that accounts for only $5 \%$ of all UCs. Owing to its relative rarity, most studies of UC-UUT have been limited to small series of cases. Here we analyzed the pathologic and clinical features of UC-UUTs in a large cohort of patients from a single institution.

Design: We retrospectively searched our pathology database and found 100 cases of UC-UUT that had been treated from 1991 to 2006. All patients had undergone radical nephroureterectomy. Slides were reviewed for pathologic analysis. Demographic and clinical information was collected from medical records. Tissue microarrays, constructed with use of three 1-mm cores from each case, were used for immunohistochemical evaluation.

Results: The patients included 66 men and 34 women with a mean age of 67 years (range, 32-91 years). The tumors were located at the ureter $(n=38)$, renal pelvis $(n=43)$, both renal pelvis and ureter $(n=12)$, and ureteropelvic junction $(n=7)$. The UCs included high grade $(n=68)$ and low grade $(n=32)$. The tumors were noninvasive in 23 cases and invasive in 77 cases, including T1 $(n=18)$, T2 $(n=21)$, T3 $(n=29)$, and T4 $(n=9)$. Compared with ureteral UC, pelvic UC showed higher grade ( $72 \%$ versus $60 \%)$ and higher stage $(63 \%$ versus $55 \%)$. Follow-up information was available for all patients for a mean period of 56 months (range, 1-238 months). 55 patients died in 39 months (mean) after surgery, and 45 patients were alive in 78 months (mean). Mortality was significantly associated with tumor grade and stage. Immunohistochemical analysis showed that GATA-3 was more frequently expressed in ureteral UC than in pelvic UC ( $45 \%$ versus $18 \%$ ). CK14 was expressed in 16 cases of UC-UUT and showed significant association with advanced stage, distant metastasis, and high mortality.

Conclusions: UC-UUT is an aggressive disease that often presents at advanced stage and is associated with poor clinical outcome. GATA-3 is expressed at different levels in ureteral and pelvic UCs and should therefore be used with caution as a biomarker in pelvic UC. CK14 is a potentially valuable biomarker that can predict cancer metastasis and patient outcome in UC-UUT.

\section{Reflex FISH Testing of Subdivided Cytologically Atypical Urine} Specimens

Ryan Glass, Dwayne Breining, Rubina Cocker. North Shore Long Island Jewish, New Hyde Park, NY.

Background: FISH UroVysion remains an important ancillary test for the detection of high grade urothelial carcinoma (HGUC) due to its reported high sensitivity. Our recent study investigated its use with the Paris System, particularly the new atypical subcategories: Atypical Urothelial Cells uncertain significance (AUC-US) and cannot exclude HGUC (AUC-H). We found that FISH can be optimized by limiting use to AUC-US patients. The current study aims to confirm this and evaluate variations in specific cytogenetic abnormalities.

Design: A laboratory system search was conducted from 2007-2015 to identify all urine cytologies with concurrent FISH testing and biopsy results within six months. Specimens reported as "atypical" were manually reviewed and reclassified into AUC-US and AUS-H based on Paris system criteria. Biopsy outcomes were compared between the reclassified AUC-US and AUC-H groups, likelihood ratios were calculated based on FISH results, and cytogenetic abnormalities were tabulated.

Results: The search identified 244 cases including 72 atypical specimens ( 57 reclassified as AUC-US, 15 as AUC-HG). $40 \%$ of AUC-H versus $19.3 \%$ of AUC-US cases had HGUC on biopsy.

\begin{tabular}{|c|c|c|c|c|}
\hline Cytology & $\begin{array}{l}\text { Pretest Prob } \\
\text { of HGUC }\end{array}$ & \multicolumn{2}{|c|}{$\begin{array}{l}\text { Likelihood } \\
\text { Ratio }\end{array}$} & $\begin{array}{l}\text { Postest } \\
\text { Prob }\end{array}$ \\
\hline \multirow{2}{*}{ NMC } & \multirow{2}{*}{0.18} & + & 1.67 & 0.27 \\
\hline & & - & 0.71 & 0.14 \\
\hline \multirow{2}{*}{ AUC-US } & \multirow{2}{*}{0.19} & + & 2.44 & 0.37 \\
\hline & & - & 0.49 & 0.11 \\
\hline \multirow{2}{*}{ AUC-HG } & \multirow{2}{*}{0.40} & + & 6 & 0.80 \\
\hline & & - & 0.38 & 0.20 \\
\hline \multirow{2}{*}{ SUS } & \multirow{2}{*}{0.47} & + & 1.11 & 0.50 \\
\hline & & - & 0.83 & 0.43 \\
\hline \multirow{2}{*}{ POS } & \multirow{2}{*}{0.58} & + & 0.99 & 0.58 \\
\hline & & - & 1.07 & 0.60 \\
\hline
\end{tabular}

The greatest benefit for FISH testing occurred in AUC-US specimens (LR+ 2.44, LR0.49). Among patients positive for FISH testing, duplications of chromosome 3, 7 or 17 were present in the majority of cases.

\begin{tabular}{|l|l|l|}
\hline \multirow{2}{*}{$\begin{array}{l}\text { Cytology } \\
\text { (FISH Pos) }\end{array}$} & Biopsy & HGUC \\
\cline { 2 - 3 } & NEG & $\mathbf{9}$ \\
\hline NMC & $\mathbf{1 8}$ & 8 \\
\hline Dup & 16 & 4 \\
\hline Del & 4 & 3 \\
\hline Both & 2 & $\mathbf{7}$ \\
\hline AUC-US & $\mathbf{1 1}$ & 7 \\
\hline Dup & 11 & 1 \\
\hline Del & 1 & 1 \\
\hline Both & 1 & $\mathbf{4}$ \\
\hline AUC-H & $\mathbf{0}$ & 3 \\
\hline Dup & 0 & 1 \\
\hline Del & 0 & 0 \\
\hline Both & 0 & $\mathbf{1 2}$ \\
\hline SUS & $\mathbf{1 0}$ & 12 \\
\hline Dup & 10 & 1 \\
\hline Del & 1 & 1 \\
\hline Both & 1 & $\mathbf{1 8}$ \\
\hline POS & $\mathbf{5}$ & 18 \\
\hline Dup & 5 & 7 \\
\hline Del & 0 & 7 \\
\hline Both & 0 & \\
\hline & & 7 \\
\hline
\end{tabular}

9 p23 deletions were present in few cases, particularly those with atypical cytology, and rarely as the sole cytogenetic abnormality. 
Conclusions: Patients from the AUC-H group had double the risk of HGUC as those from the AUC-US group. FISH was helpful in identifying occult malignancies among AUC-US patients. The majority of patients with positive FISH had chromosome duplications. Of the few that had deletions, most had concomitant duplications. These data suggest that subdividing atypical specimens and limiting the use of certain FISH probes may optimize HGUC screening and FISH testing while reducing screening costs.

925 MAGI-2 Is a Sensitive and Specific Marker of Prostatic Adenocarcinoma: Comparison with AMACR

Jeffery Goldstein, Rajen Goyal, Joseph T Roland, Lan L Gellert, Peter E Clark, Giovanna A Giannico, Omar Hameed. Vanderbilt University Medical Center, Nashville, TN.

Background: Membrane-associated guanylate kinase, WW and PDZ domain-containing protein 2 (MAGI-2) is a scaffolding molecule which interacts with multiple cell adhesion molecules, receptors and the cytoskeleton. MAGI-2 gene rearrangements have been described in prostate cancer. We compared MAGI-2 and alpha-methylacyl CoA racemase (AMACR) expression by immunohistochemistry to compare their utility in the diagnosis of prostatic adenocarcinoma.

Design: Three tissue microarrays consisting of 5121 -mm cores including benign prostatic glands, high grade prostatic intraepithelial neoplasia (HGPIN), and adenocarcinoma were constructed from 78 radical prostatectomies. Immunohistochemistry for AMACR (Dako, 13h4, Carpinteria, CA, neat) and MAGI-2 (MAGI-2: HPA013650, SigmaAldrich, St. Louis, MO, 1:100) was performed. Visual (H-score) and digital (percent area multiplied by mean optical density; STAIN) image analysis were performed.

Results: MAGI-2 and AMACR expression were significantly higher in adenocarcinoma and HGPIN compared to benign (All $\mathrm{p}$ values $<0.001$ ). By image analysis, a receiver/ operator curve comparing MAGI-2 and AMACR yielded an area under the curve of 0.846 and 0.937 , respectively. At H-score cutoffs of 300 and 200, MAGI-2 was more accurate, while AMACR was more accurate at H-score of 100.

\begin{tabular}{c|lccccc} 
H Score Cutoff & Protein & Specificity & Sensitivity & PPV & NPV & Accuracy \\
\hline \multirow{2}{*}{300} & MAGI-2 & 0.988 & 0.222 & 0.949 & 0.555 & 0.601 \\
& AMACR & 1 & 0.090 & 1 & 0.532 & 0.553 \\
\hline \multirow{2}{*}{200} & MAGI-2 & 0.835 & 0.719 & 0.816 & 0.745 & 0.776 \\
& AMACR & 1 & 0.401 & 1 & 0.634 & 0.706 \\
\hline \multirow{2}{*}{100} & MAGI-2 & 0.311 & 0.946 & 0.583 & 0.850 & 0.631 \\
& AMACR & 0.977 & 0.731 & 0.968 & 0.790 & 0.856 \\
\hline
\end{tabular}

In cores with coexistent malignant and benign glands, MAGI-2 was more accurate than AMACR (95\% vs 91\%, respectively). MAGI-2 staining was discriminatory in all 4 cases where AMACR was not.

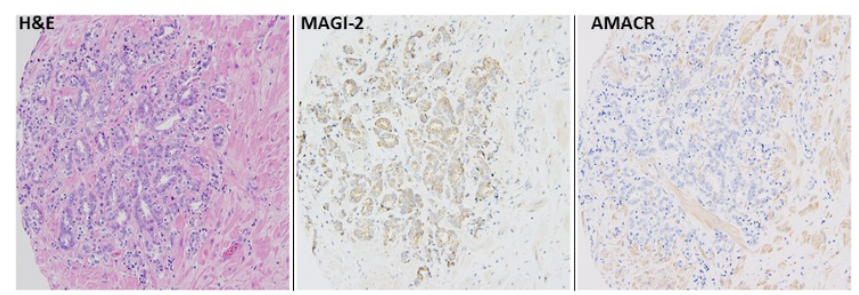

Infiltrative glands (H\&E, left panel), appropriately stained by MAGI-2 (middle), while AMACR is negative (right).

Conclusions: MAGI-2 is less specific than AMACR for the diagnosis of adenocarcinoma, however its superior sensitivity suggests it could be a useful adjunct when AMACR is negative.

926 Human Epidermal Growth Factor Receptor 2 Expression in Micropapillary Urothelial Carcinoma of the Bladder: An Analysis of 27 Cases

Abigail L Goodman, Adeboye O Osunkoya. Emory University School of Medicine, Atlanta, GA.

Background: The significance of human epidermal growth factor receptor 2 (HER2) overexpression in breast cancer is well established, and these patients are subsequently treated with Trastuzumab. However, only few large studies have analyzed HER2 expression in invasive high grade urothelial carcinoma (UCA). Micropapillary UCA is an aggressive variant of UCA, which usually presents at an advanced stage and is associated with a poor prognosis. In this study, we sought to analyze the expression of HER2 in a large cohort of Micropapillary UCA.

Design: A search was performed through our Urologic Pathology files and expert consult files of the senior author for cases of micropapillary UCA of the bladder. Only cases with available tissue blocks were included. HER 2 expression was determined by immunohistochemistry. HER2 immunostaining for intensity and percent of neoplastic cells staining were evaluated. HER2 expression was considered positive if there was a staining intensity of $2+$ or greater in at least $10 \%$ of neoplastic cells. Statistical analysis was performed using the Mann-Whitney Test, Kruskal-Wallis Test, or ANOVA as appropriate.

Results: Twenty-seven cases were identified. The mean patient age was 68 years (range: 39-87 years). There were 26 male patients and 1 female patient. Pathologic stages were as follows: pT3 $(11 / 27,41 \%)$, pT2 $(10 / 27,37 \%)$, pT1 $(5 / 27,18 \%)$ and pTa $(1 / 27,4 \%)$.
Angiolymphatic invasion was present in 15/27 (55\%) cases. Ten of $27(37 \%)$ patients developed metastatic disease. Twenty of $27(74 \%)$ cases were positive for HER2. Eleven of $15(73 \%)$ cases with angiolymphatic invasion were positive for HER2. Seven of 10 $(70 \%)$ cases that developed metastasis were positive for HER2. Six of 11 (54\%) cases with pT3 disease were positive for HER2, 8/10 (80\%) cases with pT2 disease were positive for HER2, $5 / 5(100 \%)$ cases with pT1 disease were positive for HER2 and the only case with pTa disease was positive for HER2.

Conclusions: In our study, HER 2 was overexpressed in $74 \%$ of patients with micropapillary UCA of the bladder. In our cohort, a significant number of patients with angiolymphatic invasion also had HER2 overexpression. Interestingly, although the majority of cases with advanced disease also demonstrated positive HER2 expression, it was more predominant in cases with less advanced disease. It is therefore highly conceivable that both patients with early or advanced micropapillary UCA of the bladder, including those with angiolymphatic invasion may benefit from Trastuzumab therapy.

\section{Morphologic and Immunohistochemical Correlates of 99mTc-} sestamibi SPECT/CT Imaging Findings of Renal Neoplasms

Michael A Gorin, Steve P Rowe, Mehrbod S Javadi, Mohamad E Allaf, Jonathan I Epstein, Alexander Baras. Johns Hopkins, Baltimore, MD.

Background: In a recent publication from our group, we found that single-photon emission computed tomography/x-ray computed tomography (SPECT/CT) utilizing the mitochondrial imaging agent ${ }^{99 \mathrm{~m}} \mathrm{Tc}$-sestamibi allowed for the highly accurate ( $>$ 95\%) differentiation of clear cell (ccRCC) and papillary renal cell carcinomas (pRCC) apart from benign oncocytomas and hybrid oncocytic/chromophobe tumors (HOCTs). Chromophobe renal cell carcinomas, however, showed a high degree of variability in terms of radiotracer uptake. This study aims to identify histomorphologic and/or immunohistochemical features of chromophobe renal cell carcinoma that could be used to explain these findings.

Design: From our study cohort, which included 50 patients imaged with ${ }^{99 \mathrm{~m}} \mathrm{Tc}$-sestamibi SPECT/CT prior to surgical resection, cases of chromophobe RCC were reviewed for the presence of eosinophilic features in a blinded manner. In addition, randomly selected cases from a number of renal tumor histologies were stained for a mitochondrial content as well as expression of the multidrug resistance (MDR) protein, a membraneassociated protein responsible for eliminating ${ }^{99 \mathrm{~m}} \mathrm{Tc}$-sestamibi from cells. Finally, a tissue microarray of the full imaged cohort was constructed.

Results: Interestingly, the two cases of chromophobe RCC that showed high levels of ${ }^{99 \mathrm{~m}} \mathrm{Tc}$-sestamibi uptake similar to that seen with oncocytomas were characterized as eosinophilic variants on blinded pathologic review. The initial staining of randomly selected cases for mitochondrial content and MDR protein expression level suggests that there exists a varied constellation of staining across the spectrum of renal neoplasms examined. The degree of radiotracer uptake across this spectrum of renal tumors is at least in part explained by the preliminary results.

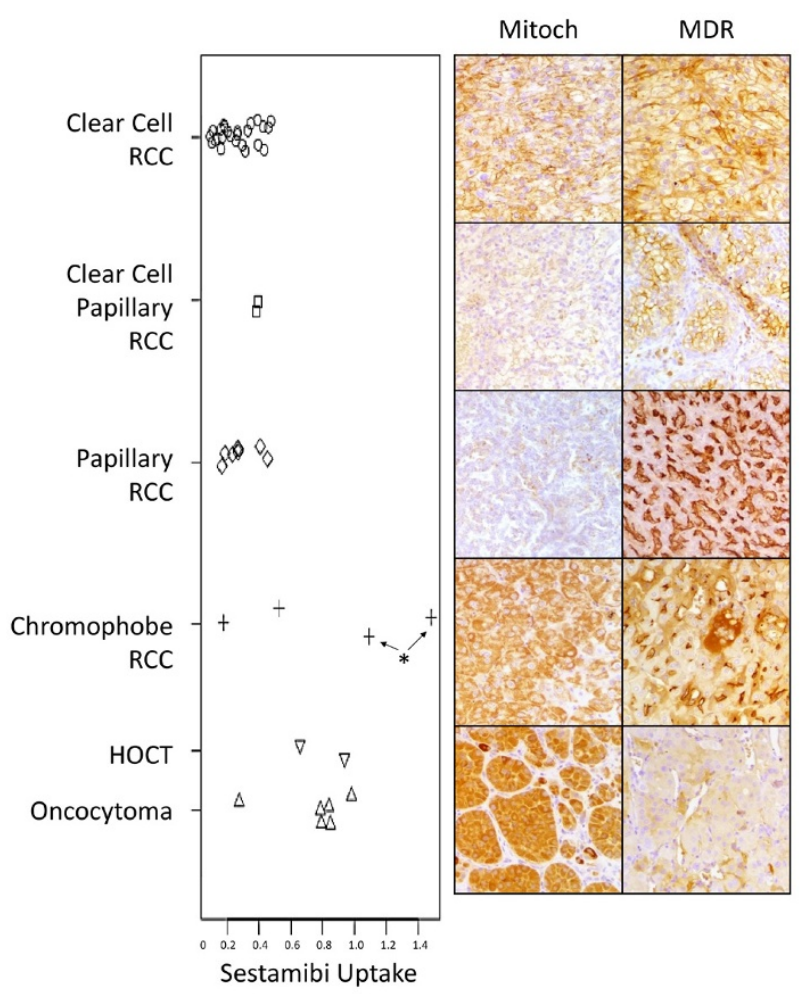

Figure 1. Histomorphologic and immunohistochemical correlates of the distribution of sestamib radiotracer uptake across a spectrum of renal neoplasm.

The staining patterns for mitochondrial content (Mitoch) and a multidrug resistance transport protein (MDR) are shown in 
Conclusions: Our preliminary findings suggest that there exist histomorphologic and immunohistochemical findings that explain the variable uptake of ${ }^{99 \mathrm{~m}} \mathrm{Tc}$-sestamibi observed in cases of chromophobe RCC. Future work aims to more thoroughly explore these findings utilizing a utilizing a TMA constructed from the study cohort.

\section{Molecular Evidence of Preneoplastic Potential of Intestinal} Metaplasia in the Urinary Bladder

Zakaria Grada, Andres Matoso, Virginia Breese, Cynthia Jackson, Ali Amin. Rhode Island Hospital/Brown University, Providence, RI.

Background: Intestinal metaplasia (IM) is an occasional finding in urinary bladder specimens. It is unclear if IM without dysplasia is a precursor of malignancy in the urinary system. This is the first study to investigate presence of mutations commonly identified in colorectal adenocarcinoma in IM.

Design: 24 random cases were retrieved from the archives of the Rhode Island Hospital Pathology Department including 7 cases of IM of bladder (one case harboring high grade dysplasia), 7 tubulovillous adenomas (TVA) of the colon, and 10 cases of high grade papillary urothelial carcinoma (UC). DNA isolation from paraffin embedded tissue sections was performed followed by mutation analysis for $B R A F, A P C, K R A S$, MET, NRAS, PIK3CA, CTNNB1, FBXW7 and TP53 using MassARRAY@ System (Sequenom Laboratories, San Diego, CA).

Results: The IM cases included 5 males and 2 females with mean age of 52 (range: $30-71 \mathrm{y})$. Clinical presentations included hematuria $(\mathrm{n}=2)$ and surveillance for urothelial carcinoma $(\mathrm{n}=5)$. In $5(71 \%)$ cases with IM no mutation was identified. One IM case revealed APC mutation and another showed NRAS mutation. Among the TVA cases, 6 of $7(85.7 \%)$ showed $K R A S$ mutation and 3 of $7(42 \%)$ showed $A P C$ mutation. Among UC cases, 1 revealed KRAS mutation, 2 had PIK3CA mutation and all cases were negative for $A P C$ mutation. Clinical follow-up for the IM patients was available with a median follow-up of 70 months. One patient - without any mutation - developed invasive bladder adenocarcinoma with metastasis to liver and lung; but neither of the 2 patients harboring mutation developed any malignancy.

Conclusions: A minority of cases with IM without dysplasia bear mutation in the genes commonly associated with colon adenocarcinoma, suggesting a premalignant potential for such lesions possibly following the colorectal pathways of carcinogenesis. A larger cohort of patients with longer follow-up is needed to better establish whether close follow-up is warranted for mutation-harboring IM of bladder.

929 Value of a Genomic Risk Classifier (GPS Score) in Relation to Histopathologic Parameters

Nancy Greenland, Bradley Stohr, Jeffrey P Simko. University of California, San Francisco, CA.

Background: While clinical and pathologic parameters are excellent predictors of patient outcomes in prostate cancer patient management, numerous attempts have been made to further improve current risk assessment predictors by including tissue-based biomarker evaluations into these algorithms. Many of these tests now have been turned into commercial products. The value of these tests is still unknown, as they have never been prospectively evaluated. Here, we attempted to correlate histologic findings from prostate biopsies to the results obtained from one of these commercial tests (the Genomic Health GPS score) with the goal of trying to identify potential utility and limitations of the test results. Specifically, for cases where the GPS score predicted either a more or less aggressive tumor than that predicted by clinicopathologic parameters alone, we retrospectively evaluated the prostate biopsy to determine if the disagreement was due to inaccurate Gleason grading, or that the test provided additional information. Design: The GPS score provided by Genomic Health is designed to identify patients at risk of aggressive pathologic findings at prostatectomy using an assay that measures expression level of 17 genes that are then incorporated into an algorithm that also considers clinicopathologic parameters. The test provides an absolute test score (GPS score) and after incorporation of clinicopathologic parameters a percent likelihood of favorable pathology at prostatectomy. We retrospectively evaluated the pathologic findings for all cases sent to this company for testing from a single institution that had results that deviated from those predicted by the NCCN risk assignment alone. For each case, the Gleason score was reassigned, the NCCN risk reassessed, and the value of the test reevaluated.

Results: Of 212 biopsies tested, 7 failed due to insufficient tumor amount, and 5 were rejected for Gleason score beyond validated test range. NCCN scores before and after testing were concordant in 152 of 200 cases tested. Of these, 30 were discordant between NCCN low and very low risk. 10 cases were intermediate risk before testing and low risk after; pathology review showed that two of these were due to misgrading. 8 cases went from low risk to intermediate after testing, and two of these were due to misgrading. Conclusions: Gene expression-based assessment (GPS score) appears to be valuable for identifying both cases that have been incorrectly graded, as well as a subset without any as of yet recognizable pathologic features, although the number of cases where the test appears to add value is low.

930 ERG/PTEN Status of Isolated PIN Occurring in Cystoprostatectomy Specimens without Invasive Prostatic Adenocarcinoma

Liana B Guedes, Carlos L Morais, Jessica Hicks, Angelo M De Marzo, Tamara Lotan. Johns Hopkins School of Medicine, Baltimore, MD.

Background: High grade prostatic intraepithelial neoplasia (PIN) is widely believed to represent a precursor to invasive prostatic adenocarcinoma. However, recent molecular studies have suggested that retrograde spread of invasive adenocarcinoma into pre-existing prostatic ducts can morphologically mimic PIN. Thus, molecular characterization of PIN occurring in radical prostatectomies or needle biopsies is likely to be partially confounded by intraductal spread of invasive adenocarcinoma.
To assess ERG/PTEN status in PIN foci likely to represent true precursor lesions, we studied isolated PIN occurring without associated invasive adenocarcinoma in cystoprostatectomies.

Design: We queried the Johns Hopkins Pathology Database for cystoprostatectomies occurring between 2009 and 2014. Of 344 cystoprostatectomies, 33\% (114/344) contained invasive prostatic adenocarcinoma in the partially submitted prostate (10 blocks/case on average) and were excluded from the study. Of the remaining cases without sampled cancer, 30\% (69/230) showed 135 separate foci of PIN and were immunostained for ERG and PTEN using genetically validated protocols.

Results: Of foci of PIN with evaluable ERG status, $8 \%$ (9/109) were positive for ERG, with $15 \%(9 / 61)$ of cystoprostatectomy specimens containing one ERG-positive focus of PIN. PTEN loss was present in $1 \%(1 / 88)$ of evaluable PIN foci or $2 \%(1 / 60)$ of all specimens containing PIN.

Conclusions: ERG expression occurs in up to $15 \%$ of cystoprostatectomy specimens containing isolated PIN without associated invasive adenocarcinoma, while PTEN loss is exceedingly rare. Though we cannot entirely rule out the presence of small unsampled foci of invasive prostatic adenocarcinoma in these cases, these PIN foci are unlikely to represent retrograde spread of invasive tumor. Thus, ERG rearrangement, but not PTEN loss, may occur early during prostatic tumorigenesis.

\section{Analytic Validation of RNA In Situ Hybridization (RISH) for AR and} AR-V7 Expression in Human Prostate Cancer

Liana B Guedes, Carlos L Morais, Qizhi Zheng, John Isaacs, Emmanuel S Antonarakis, Angelo M De Marzo, Jun Luo, Tamara Lotan. Johns Hopkins School of Medicine, Baltimore, MD

Background: RNA expression of androgen receptor (AR) splice variants may be a biomarker of resistance to AR-targeted therapies in castrate resistant prostate cancer (CRPC). Here, we analytically validated our previously reported (Antonarakis et al, NEJM, 2014) RNA in situ hybridization (RISH) assay for total AR and AR-V7 in formalin fixed paraffin embedded (FFPE) prostate tumors.

Design: We used prostate cell lines and xenografts to validate a chromogenic RISH assay to detect RNA containing AR exon 1 (AR-E1, surrogate for total AR RNA species) and cryptic exon 3 (AR-CE3, surrogate for AR-V7 expression). RISH signals were quantified with two image analysis software platforms in primary tumors and CRPC, comparing to known AR and AR-V7 status by immunohistochemistry and RT-PCR where available. Results: Across all samples tested, both AR-E1 and AR-CE3 RISH signals were localized in nuclear punctae, in addition to the expected cytoplasmic speckles. The quantified RISH assay correlated with total AR and AR-V7 levels by RT-PCR in cell lines and xenografts. AR-E1 and AR-CE3 expression levels were highly correlated in all samples $\left(\mathrm{R}^{2}=0.693-0.991\right)$ and were highest in a subset of CRPC metastases and cell lines. AR-E1 was weakly correlated with quantified AR protein levels in primary tumors $\left(\mathrm{R}^{2}=0.232\right)$. Interestingly, mean AR-E1 expression was 2.7 and 1.7-fold higher in primary tumor glands compared to admixed benign glands in two independent cohorts $(\mathrm{p}=0.009$ and $\mathrm{p}=0.04)$. CE3 expression showed a similar trend.

Conclusions: RISH for AR-E1 and AR-CE3 are sensitive and specific assays and quantified RISH correlates with total AR and AR-V7 RNA levels in cell lines and xenografts. Expression of AR-E1, a surrogate for total AR RNA, is higher in primary tumor cells compared to benign glands. Dedicated clinical studies will be required to further validate these assays as prognostic or predictive biomarkers.

932 PNL2: An Adjunctive Biomarker for Renal Angiomyolipomas and PEComas

Previn Gulavita, Christopher Fletcher, Michelle Hirsch. Brigham and Women's Hospital, Boston, MA.

Background: Angiomyolipomas (AML) and Perivascular Epithelioid Cell Tumors (PEComa) are members of the MiTF family of tumors, and occur not infrequently in the kidney, most often sporadically or as part of the Tuberous Sclerosis Complex. Traditionally, immunohistochemical melanoma markers, such as HMB45 and MelanA have been used to support the diagnosis of these lesions; however, low sensitivity due to a frequent focal staining pattern renders interpretation difficult in some cases. PNL2 is a sensitive and specific biomarker for melanoma; PNL2 has also been shown to be positive in soft tissue AMLs and other PEComas. The goal of this study was to determine whether or not PNL2 may be a better biomarker than HMB45 and MelanA for AMLs and PEComas in the kidney.

Design: Immunohistochemical staining for PNL2 was performed on 191 tumors (49 whole mount sections, 142 on a tissue microarray), including 22 AMLs, 3 epithelioid AMLs (EpAML), 3 sclerosing PEComas, and 6 malignant PEComas, 6 Xp11.2 translocation renal cell carcinomas (RCC), 111 clear cell RCCs, 16 papillary RCCs, 9 chromophobe RCCs, 3 mucinous tubular and spindle cell RCCs, 2 clear cell tubulopapillary RCCs, 2 collecting duct RCCs, 1 renal angiomyoadenomatous tumor, 1 oncocytoma, 2 mixed epithelial stromal tumors, 1 dedifferentiated liposarcoma, 2 leiomyosarcomas, and 1 solitary fibrous tumor. HMB- 45 and MelanA were also evaluated in a subset of the MiTF tumors. Any presence of PNL2 staining in tumor cells was considered a positive result.

Results: Overall, $85 \%$ of AMLs and PEComas were positive for PNL2, compared to $81 \%$ and $88 \%$ with HMB 45 and MelanA, respectively. However, in $55 \%$ of cases PNL2 stained more extensively when compared to HMB45. In contrast, the extent of staining for PNL2 and MelanA in most cases, when present, was nearly the same. See the Table below for the breakdown of staining by MiTF tumor subtype. All remaining renal tumors and RCCs, except one Xp11.2 translocation RCC, were negative for PNL2. 


\begin{tabular}{|l|l|l|l|}
\hline & PNL2 (\%) & HMB45 (\%) & MelanA (\%) \\
\hline AML (N=22) & 86 & 86 & 100 \\
\hline EpAML (N=3) & 67 & 67 & 67 \\
\hline Malignant PEComa (N=6) & 83 & 67 & 50 \\
\hline Sclerosing PEComa (N=3) & 100 & 100 & 0 \\
\hline
\end{tabular}

\begin{tabular}{|l|l|l|l|l|}
\hline $\begin{array}{l}\text { Diagnosis of } \\
\text { SFT }\end{array}$ & $\begin{array}{l}\text { Sensitivity } \\
(\%)\end{array}$ & $\begin{array}{l}\text { Specificity } \\
(\%)\end{array}$ & $\begin{array}{l}\text { Positive Predictive } \\
\text { Value(\%) }\end{array}$ & $\begin{array}{l}\text { Negative Predictive } \\
\text { Value }(\%)\end{array}$ \\
\hline Only STAT6+ & 90.91 & 75 & 71.43 & 92.31 \\
\hline Only ALDH1+ & 83.33 & 80 & 76.92 & 85.71 \\
\hline $\begin{array}{l}\text { STAT6 + and } \\
\text { ALDH1+ }\end{array}$ & 90.91 & 100 & 100 & 93.75 \\
\hline
\end{tabular}

Conclusions: PNL2 is a highly sensitive and specific marker for renal AML and PEComas when compared to non-MiTF renal tumors and RCCs. While sensitivity is similar to that of HMB45 and MelanA in conventional AMLs, it may be a more reliable marker in rarer subtypes such as sclerosing and malignant PEComa. Additionally, for cases that are morphologically suspicious for AML/PEComa, but negative for HMB45 and MelanA, PNL2 may be a useful adjunct biomarker.

\section{Morphologic and Molecular Evaluation of Thyroid-Like Follicular} Carcinoma of the Kidney

Previn Gulavita, Ming Zhou, Mahul B Amin, Deepika Sirohi, Elizabeth Garcia, Paola Dal Cin, Sean R Williamson, Michelle Hirsch. Brigham and Women's Hospital, Boston, MA; New York University, New York, NY; Cedars Sinai Medical Center, Irvine, CA; Henry Ford Hospital, Detroit, MI.

Background: Thyroid-like follicular carcinoma of the kidney (TLFCK) is a rare yet emerging subtype of renal cell carcinoma (RCC) that has morphologic resemblance with thyroid neoplasms. Some believe that TLFCK is a variant of papillary RCC, but there is limited supporting evidence for this association. Additionally, morphologic similarity to mesonephric carcinoma of the gynecologic tract (gyn-MC) raises the possibility that TLFCK may be of mesonephric origin.

Design: 10 renal neoplasms, plus 1 renal mesonephric remnant (MR), with morphologic features similar to that seen in thyroid or gyn-MCs were evaluated morphologically, immunophenotypically, and with next generation sequencing (NGSeq) to gain a better understanding of the morphologic features and genetic/molecular alterations.

Results: Tumors presented in patients ranging 15-77 years old (1:1 M:F), and ranged from 1 to $16.5 \mathrm{~cm}$ (median $3.2 \mathrm{~cm}$ ). 8 cases were pTla, $2 \mathrm{pT}$. All 10 tumors appeared well circumscribed and had at minimum a tubular architecture, 9 including micro and macrofollicular formation with luminal eosinophilic material. 4 cases also had papillary features. 9 cases demonstrated nuclear grooves; one had prominent nuclear clearing and pseudoinclusions. All 10 cases were PAX8 and EMA positive, and TTF1 negative. Similar to gyn-MCs and 1 MR, 3 cases were positive for GATA3, CD10 and calretinin; 2 of these were $\mathrm{CK} 7$ and AMACR negative. 6 GATA3 negative cases were $\mathrm{CK} 7$ and AMACR positive; $\mathrm{CD} 10$ and calretinin were also negative. 1 case was negative for all markers except CD10. NGSeq was performed on 7 cases. A total of 40 gene mutations were identified (range 5-11 per case); 6 mutations recurred in 2 cases each. Copy number variation (CNV) was present in 5 cases, including 2 cases with $+7 /+17$. Other chromosomal gains included $+2,+8,+12$ and +16 . No chromosomal loss was seen. 2 cases demonstrated focal copy number gain at the region of SOX9, BCL2, and MET. One pT3a case presented with LN metastases. All patients are negative for active disease (up to 36 months follow-up).

Conclusions: These findings demonstrate that RCCs with thyroid-like morphology demonstrate immunoprofiles and chromosomal alterations that overlap with mesonephric and papillary RCC. Interestingly, trisomy 7, 12, 16 and 17 has been previously reported in presumed TLFCK. Additional investigation of the molecular alterations in TLFCK may help gain a better understanding of the pathophysiology of these relatively indolent tumors

934 Diagnostic Value of Dual Reactivity of STAT6 and ALDH1 in the Differential Diagnosis of Solitary Fibrous Tumor vs Stromal Tumor of Unknown Malignant Potential of the Prostate

Gunes Guner, Stephania M Bezerra, Diana Taheri, David J Zahavi, Maria Angelica Mendoza Rodriguez, Rajni Sharma, Justin A Bishop, Jonathan I Epstein, George J Netto. Johns Hopkins Medical Institution, Baltimore, MD; Isfahan University of Medical Sciences, Isfahan Kidney Diseases Research Center, Isfahan, Islamic Republic of Iran. Background: The diagnosis of solitary fibrous tumor (SFT) in the prostate can be challenging, especially on small biopsies. Prostatic stromal tumors of unknown malignant potential (STUMP) and SFT may share some overlapping morphologic features and both express CD34. NAB2-STAT6 gene fusions have recently been identified in SFT of various sites, the fusion leads to nuclear translocalization of the STAT6 protein. Immunohistochemical nuclear staining of the STAT6 antibody is now considered an adjunct for SFT diagnosis. We evaluated the utility of STAT6 and an emerging stemness marker ALDH1 immunostains in the differential diagnosis of SFT vs stromal lesions of the prostate.

Design: Thirty-two cases (12 needle biopsies, 13 RRP and 7 TURP) were retrieved from surgical pathology and consultation archives (1995-2015). They included 16 STUMPs, 12 SFTs, 4 low grade prostatic stromal sarcomas. Sections were stained with polyclonal STAT6 antibody (Santa Cruz, S20, 1:100) and monoclonal ALDH1 antibody (BD Biosciences, Clone 44, 1:250). Unequivocal nuclear staining for STAT6 and cytoplasmic ALDH1 staining were considered positive.

Results: Ten of 11 evaluable SFTs demonstrated nuclear STAT6 positivity. Four of 15 evaluable STUMP cases were STAT6 positive, in the later cases, staining tended to be weak or focal. ALDH1 positivity was seen in 10/12 evaluable SFTs, while 3/15 STUMPs were ALDH1-positive. All four low grade stromal sarcomas were STAT6 negative and 2 were ALDH1-positive.

The sensitivity and specificity of STAT6 alone for the diagnosis of SFT was $90.91 \%$ and $75 \%$, respectively. A combination of STAT6 and ALDH1 positivity improved specificity $(100 \%)$ for the diagnosis of SFT.

Conclusions: STAT6 and ALDH1 are useful markers in the differential diagnosis of prostatic SFT vs STUMP. A combination of STAT6 and ALDH1 positivity is highly scpecific for the diagnosis of SFT while dual negativity for both markers is useful for ruling out SFT.

935 Molecular Characterization of Clinically Aggressive Micropapillary Bladder Cancer

Charles C Guo, Vipulkumar Dadhania, Li Zhang, Jolanta Bondaruk, Tadeusz Majewski, Maciej Sykulski, Weronika Wronowska, Anna Gambin, Ashish Kamat, Colin Dinney, Woonyoung Choi, David McConkey, John Weinstein, Bogdan Czerniak. The University of Texas MD Anderson Cancer Center, Houston, TX; Institute of Statistics, University of Warsaw, Warsaw, Poland.

Background: Progression of conventional urothelial carcinoma of the bladder to a tumor with unique microscopic features referred to as micropapillary carcinoma is coupled with aggressive clinical behavior signified by a high propensity for regional lymph node and distant organ metastases resulting in shorter survival. Here we report on the expression profile of micropapillary cancer and identify the signature molecular mechanisms that drive its development and aggressive behavior.

Design: We performed whole genome mRNA expression profiling and unsupervised hierarchical clustering analysis using formalin fixed paraffin embedded tissues on 43 micropapillary bladder cancers and a reference set of 89 conventional urothelial carcinomas with follow-up data. The micropapillary tumors were comprised of 35 cases in which only mRNA from the micropapillary component was available, and the remaining eight cases in which mRNA was extracted from both the conventional urothelial carcinoma and micropapillary components of the same tumors.

Results: We identified that the striking feature of micropaillary carcinoma was widespread dysregulation of gene expression resulting in upregulation of multiple oncogenic pathways affecting cell proliferation and signal transduction. In contrast to conventional urothelial carcinomas which, based on their expression, can be equally divided into luminal and basal subtypes, micropapillary cancers were almost exclusively luminal. Consistent with their luminal nature, micropapillary cancers were characterized by active PPAR $\gamma$ and estrogen receptor transcriptions which were previously shown to drive the expression pattern of luminal bladder cancer. They also showed suppression of p63 target genes which were typically activated in basal cancers. Similar to conventional luminal urothelial carcinomas, a subset of micropapillary cancer exhibited the activation of the wild-type p53 downstream genes and represented the most aggressive variant of the disease with the shortest survival among the molecular subtypes. The signature feature of micropapillary cancer was the downregulation of miRNA296-5p and the activation of chromatin-remodeling complex RUVBL1.

Conclusions: We identified several putative molecular mechanisms that contribute to the development of micropapillary cancer and its clinical behavior. Our observations have important implications for prognostication and future development of more effective therapies for this unique variant of bladder cancer.

936 Urinary Bladder Paragangliomas: Analysis of Succinate Dehydrogenase and Outcome

Sounak Gupta, Jun Zhang, Lori A Erickson. Mayo Clinic, Rochester, MN.

Background: Paragangliomas (PGL) of the Urinary Bladder (UB) are rare tumors and can arise as a part of the hereditary paraganglioma-pheochromocytoma syndrome due to underlying mutations in the succinate dehydrogenase (SDH) genes. The SDH complex, comprising of subunits A, B, C, D and SDHAF2, plays an important role in Krebs cycle and serves as a tumor suppressor. Germline mutations coupled with a somatic mutation of any of these subunits leads to SDH deficiency and can be screened for, by absence of immunohistochemical detection of SDHB. In this study of 11 cases with long-term follow up, we sought to characterize the clinicopathologic features of malignant UB-PGLs.

Design: The study involved 11 cases of UB-PGLs diagnosed between 1972 and 2013 at our institution. Clinical follow up information was available for $9 / 11$ cases with a mean follow up of 124 months (13-281). The cases were reviewed and graded based on GAPP criteria (maximum score of 10pts) which included: histological pattern, cellularity, comedo-necrosis, capsular/vascular invasion, Ki67 labeling and catecholamine type IHC was performed on representative tissue blocks for Ki67 and SDHB. Proliferative index was calculated by manual quantification of Ki-67 positive cells at selected hotspots using ImageJ (NIH). The medical record was accessed for documentation of germline SDH mutation status.

Results: In our series, UB-PGLs had a female predilection (8/11 cases) and $5 / 11$ cases were malignant. Patients with malignant UB-PGLs tended to be younger (mean age at diagnosis: 43 vs $49 \mathrm{yrs})$, have larger lesions $(5.8 \mathrm{vs} 1.5 \mathrm{~cm})$ and presented with catecholamine excess (norepinephrine alone or norepinephrine and dopamine, $4 / 4 \mathrm{vs}$ $2 / 6$ patients with benign UB-PGLs). Patients with malignant UB-PGLs showed a higher mean Ki67 proliferation rate $(4.2 \%$ vs $1.3 \%$ ) as well as a higher GAPP grade (mean score of 5.5 vs 3.8). IHC for SDHB expression revealed loss of expression in $2 / 6$ cases of benign PGLs compared to $4 / 5$ patients with malignant PGLs. Interestingly, of these 4 patients, 2 had a documented mutation of SDHB, 1 patient had a SDHC mutation and another patient had a history of familial disease without mutation analysis being performed. No SDH mutation analysis was performed for the benign cohort. 
Conclusions: Our study indicates SDH mutation status and loss were strongly predictive of malignant behavior besides younger age at diagnosis, larger tumor size and two components of the GAPP grading system: higher Ki67 proliferation rate and catecholamine type.

\section{Paraganglioma Associated Renal Lesions}

Sounak Gupta, Jun Zhang, Lori A Erickson. Mayo Clinic, Rochester, MN.

Background: Germline mutations, followed by a "second hit" to the remaining allele of the von Hippel-Lindau (VHL) and succinate dehydrogenase (SDH) genes have been associated with the development of pheochromocytomas (PCC) and paragangliomas (PGL) as part of the VHL and hereditary paraganglioma-pheochromocytoma syndromes (hPGL-PCC). Both syndromes are associated with the development of renal neoplasms: clear cell (CC) renal cell carcinoma (RCC) in VHL syndrome and a broader spectrum of renal neoplasms in hPGL-PCC. In this study, we have characterized the clinicopathologic features of patients with PCCs/PGLs and associated renal lesions.

Design: The study involved 13 cases of PCCs (9) and PGLs (4) diagnosed between 1981 and 2014 at our institution and clinical follow up information was available for 12/13 cases, with a mean follow up of 73 months (4-215). Both the primary and associated renal lesion were reviewed and subjected to IHC for SDHB. The PCCs/PGLs were graded based on GAPP criteria (maximum score of 10pts) which included: histological parameters, Ki67 labeling and catecholamine type. The medical record was accessed for documentation of germline VHL \& SDH mutation status.

Results: The spectrum of associated renal lesions included 2 PGLs, 9 CC RCCs (Grade 1-2), 1 tubulocystic RCC and 1 case of multiple papillary adenomas. These were diagnosed concurrently with PGLs and PCCs for 7 cases, while the remaining 6 cases were diagnosed on average, 27 months prior. $3 / 13$ patients had metastatic PCCs/PGLs and tended to be younger ( $41 \mathrm{vs} 48 \mathrm{years})$ and have larger tumors $(10.1 \mathrm{vs} 5.7 \mathrm{~cm})$ with only $1 / 3$ cases demonstrating SDHB mutation and loss of expression compared to $2 / 9$ benign cases. 3 patients had VHL syndrome and presented with PCCs and CC RCCs, with none developing metastatic disease and only 1/3 PCCs showing loss of SDHB. Renal PGLs and CC RCC demonstrated weak SDHB expression ( $0-1+)$ while papillary adenomas and tubulocystic RCCs showed strong expression (2-3+). Amongst the renal lesions, 3 patients had metastatic CC RCCs with only 1 case showing loss of SDHB without concurrent loss in the associated PCC. Overall, the GAPP score had limited utility in separating benign and metastatic tumors (mean score: 3.4 vs 5).

Conclusions: Renal lesions associated with PGLs tend to be diagnosed concurrently or on average 27 months prior, with CC RCCs being the most common. Up to a third of these patients have VHL syndrome or mutations in SDH. However, in this set of cases neither SDHB expression status nor GAPP criteria appear to reliably separate patients with benign and metastatic disease.

938 Study of KRAS and BRAF Mutations among Different Tumor Types in the Lower Urinary Tract

Jen-Fan Hang, Chin-Chen Pan. Taipei Veterans General Hospital, Taipei, Taiwan. Background: The mitogen-activated protein kinase (MAPK) signaling pathway is known to be activated in various kinds of somatic cancers. The most common causes of activation in this pathway are point mutations involving $K R A S$ and $B R A F$ genes. However, the frequency of $K R A S$ and $B R A F$ mutations among different tumor types in the lower urinary tract is inconsistent and not well-established in the literature. For example, KRAS mutation has been reported from $4-29 \%$ in the urothelial carcinomas, while data is limited for adenocarcinomas of urachus and urinary bladder. The aim of this study was to investigate the KRAS and BRAF mutations in several types of lower urinary tract cancers.

Design: Forty-eight high-grade urothelial carcinomas, 20 high-grade urothelial carcinomas with glandular differentiation, 39 low-grade urothelial carcinomas, 7 papillary urothelial neoplasms with low malignant potential, 5 primary adenocarcinomas of urinary bladder and 13 urachal adenocarcinomas were recruited for this study. The clinical information was obtained from the medical records and the original H\&E slides were reviewed. The selected tumorous areas of each case was microdissected and subjected to DNA extraction, polymerase chain reaction and direct sequencing of $K R A S$ (exon 2) and BRAF (exon 15) genes.

Results: There were $5(38.5 \%)$ and $1(20 \%)$ cases harboring KRAS mutations in the adenocarcinomas of urachus and urinary bladder respectively. On the other hand, only $6(5.3 \%)$ cases with $K R A S$ mutations were found in the usual urothelial neoplasms. The KRAS mutations were found mainly at codon 12 and 13 and were significantly more frequent in the adenocarcinomas than in the usual urothelial neoplasms ( $\mathrm{p}<0.001$, Chi square). The BRAF D594G mutation was noted in 1 primary adenocarcinoma of urinary bladder only.

\begin{tabular}{|l|l|l|}
\hline Tumor types (n) & $\begin{array}{l}\text { KRAS mutation } \\
(\%)\end{array}$ & $\begin{array}{l}\text { BRAF mutation } \\
(\%)\end{array}$ \\
\hline Urachal adenocarcinoma (13) & $5(38.5)$ & $0(0)$ \\
\hline Primary adenocarcinomas of urinary bladder (5) & $1(20)$ & $1(20)$ \\
\hline High-grade urothelial carcinoma (48) & $4(8.3)$ & $0(0)$ \\
\hline $\begin{array}{l}\text { High-grade urothelial carcinomas with glandular } \\
\text { differentiation (20) }\end{array}$ & $0(0)$ & $0(0)$ \\
\hline Low-grade urothelial carcinoma (39) & $2(5.1)$ & $0(0)$ \\
\hline $\begin{array}{l}\text { Papillary urothelial neoplasms with low malignant } \\
\text { potential (7) }\end{array}$ & $0(0)$ & $0(0)$ \\
\hline
\end{tabular}

Conclusions: The KRAS mutations are more frequently seen in the adenocarcinomas of urachus and urinary bladder than the usual urothelial neoplasms of urinary bladder, while the $B R A F$ mutations are rarely present in these tumors.
939 Comprehensive Molecular Analysis of Small Cell Carcinoma of the Bladder

Xueli Hao, Matthew T Chang, Alexander V Penson, Neil Desai, Gopa Iyer, Victor E Reuter, Satish K Tickoo, Anuradha Gopalan, Sahussapont J Sirintrapun, Samson Fine, Ying-Bei Chen, Jonathan E Rosenberg, Anne M Schultheis, Britta Weigelt, Sasinya N Scott, Eugen K Cha, Dean F Bajorin, Michael F Berger, Bernard H Bochner, Guido Dalbagni, David B Solit, Barry S Taylor, Hikmat A Al-Ahmadie. Memorial Sloan Kettering Cancer Center, New York, NY; UT Southwestern, Dallas, TX.

Background: Small cell carcinoma of the bladder (SCCB) is a rare, high grade neuroendocrine neoplasm with aggressive clinical course and poor outcome. It is often associated with classical urothelial carcinoma (UC). A comprehensive molecular analysis of this tumor is lacking.

Design: From 145 histologically confirmed cases of SCCB, we performed a combination of whole genome, whole exome, (WGS, WES) and deep targeted sequencing (MSKIMPACT) of tumor and matching normal from 61 cases, including a subset of cases where components of small cell and non-small cell histology were dissected and sequenced separately. Integrating molecular, clinical and pathway data along with comparative genomic analyses of the TCGA $(n=131)$, our institutional prospective sequenced UC $(n=167)$ and published results of small cell carcinoma of the lung (SCCL, $\mathrm{n}=110$ ), we identified lineage- and histology-specific lesions and significant differences in the prevalence of specific pathway aberrations.

Results: Median patient age at presentation was 69 years (range, 38 to 94 y) with a male to female ratio of $4: 1$.

Mutations in the TERT promoter (93\%) and TP53 (90\%) and inactivation of RB1 $(87 \%)$ were found in nearly all samples, suggesting G1/S cell cycle checkpoint loss is an obligatory step in SCCB pathogenesis. Overall the molecular profile of SCCB was similar to that of $\mathrm{UC}$, with high prevalence of mutations in chromatin remodeling genes (ARID1A, KMT2A, KMT2C, KMT2D, CREBBP and EP300). Notably, mutations in $K D M 6 A$ and $F G F R 3, C C N D 1$ amplifications and $C D K N 2 A$ deletions were significantly fewer in SCCB compared to UC. More SCCB had E2F3 amplification (34\%) and ERBB2 mutations ( $23 \%$ ) than seen in UC, $15 \%$ and $13 \%$ respectively. $2 / 3$ 's of SCCB had evidence of whole genome duplication.

The main differences between SCCB and SCCL were the lack of TERT promoter mutations in SCCL and very low frequency of mutations in chromatic remodeling genes and $E R B B 2$ or $E 2 F 3$ amplification in SCCL. In almost all cases of SCCB, the distribution of trinucleotide mutation context was associated with mutation by the APOBEC enzyme, while in SCCL it was universally associated with mutagens found in tobacco smoke. Conclusions: SCCB shares many genetic alterations with classic UC. Loss-of-function mutations in RBI and TP53 and TERT promoter mutations are present in the vast majority of SCCB, often co-occurring with $E 2 F 3$ locus amplifications. Frequent $E R B B 2$ mutations may represent a potential therapeutic target in patients with SCCB. Molecular profiling suggests that the mechanism of mutagenesis between SCCB and SCCL is different.

940 Succinate Dehydrogenase (SDH)-Deficient Renal Cell Carcinoma, a Report of 7 Cases with an Emphasis on High Grade Morphologic Features Xueli Hao, Hikmat A Al-Ahmadie, Anuradha Gopalan, Sahussapont J Sirintrapun, Samson Fine, Satish K Tickoo, Victor E Reuter, Ying-Bei Chen. Memorial Sloan Kettering Cancer Center, New York, NY.

Background: SDH-deficient renal cell carcinoma (RCC) is a rare entity that is often recognized by its monomorphic tumor cells with abundant eosinophilic flocculent cytoplasm, intracytoplasmic inclusions and round to oval low-grade nuclei in nested and tubular patterns. SDH-deficient RCC with high grade morphologic features has been reported, but is relatively less recognized. Here we report our institutional experience with SDH-deficient RCC with high grade features, with clinical correlation.

Design: We retrospectively identified seven cases of SDH-deficient RCC from 2011 to 2014 from the pathology archives of our institution. Immunohistochemistry (IHC) for SDHB and SDHA were performed in all cases. Morphologic features assessed included cytoplasm, nucleolar grade, architecture, necrosis and lymphovascular invasion.

Results: Mean patient age at presentation was 30 years (range, 18 to $54 \mathrm{y}$ ) with a male to female ratio of 4:3. Tumor size varied from $3.5-14 \mathrm{~cm}$ (median $8.7 \mathrm{~cm}$ ). Nucleolar (nuclear) grade was 3-4 (n=5) or $2(n=2)$. Tumor stage was pT3-pT4 $(n=4)$, pT1a-pT2 $(n=2)$, metastasis in one patient. Classic low grade morphology was seen in 2 tumors. In five tumors, tumor cells had prominent nucleoli (nucleolar grade of 3 or 4), coarse chromatin, irregular nuclear membranes, and abundant dense eosinophilic cytoplasm. Areas mimicking HLRCC-carcinoma with focal perinuclear halos were seen in two of five patients. Solid/diffuse pattern was predominant with focal nested or tubulocystic features. Sarcomatoid features $(2 / 5)$ and necrosis (3/5) were also present. By IHC, SDHB expression was lost in tumor cells $(7 / 7)$, while SDHA expression was retained in all cases (7/7). Germline mutations in $S D H B$ were confirmed in five patients; information is not available in two patients. Mean clinical follow up was 10 months (range, 1-39 m). Four of the five patients with high grade morphology had metastatic disease. These patients died of disease after a mean follow up of 14 month (range, 1-39 m). The two patients with low grade morphology were alive after 7 months and 1 month follow up, respectively.

Conclusions: SDH-deficient RCC encompasses a larger morphologic spectrum than the typical low grade histology and should be included in the differential diagnosis of high grade RCC, especially HLRCC-carcinoma given the patient's age and morphologic overlaps. SDH-deficient RCC appears to behave very aggressively when harboring high grade morphologic features. 
941 Discrepancy Between Local and Central Pathological Review of Radical Orchiectomy Specimens

Saul E Harari, Daniel J Sassoon, John Eble, Thomas M Ulbright, David J Grignon, Mohammed Idrees, Costantine Albany, Timothy A Masterson, Nasser H Hanna, Richard $S$ Foster, Lawrence H Einhorn, Liang Cheng. Indiana University School of Medicine, Indianapolis, IN

Background: This study compares the experience at a large academic institution with a uniquely high volume of orchiectomy cases versus that of other hospitals.

Design: A database search was conducted to collect orchiectomy cases that were sent to our institution for treatment of testicular cancer from 2014 to 2015. Cases sent for consultation without final diagnosis from outside hospitals were excluded. One hundred and one consecutive cases were evaluated for comparison of final diagnosis between the outside institution and our hospital.

Results: Of the 101 cases reviewed 48 (48\%) had identical stage and matching histologic subtypes. 72 cases had identical stage; 65 cases had identical histological subtypes. 17 cases were downstaged by our institution: 14 cases from T2 to T1, 2 cases from T3 to T2, and 1 case from T3 to T1. Conversely, 7 cases were upstaged by our institution, all from $\mathrm{T} 1$ to $\mathrm{T} 2$. The following subtypes were not recognized based on the original pathology reports: yolk sac tumor ( 15 cases), teratoma (8 cases), embryonal carcinoma ( 6 cases), and seminoma ( 5 cases). On the other hand, outside pathology reports included the following histologic subtypes that were not reported in our final diagnosis: choriocarcinoma ( 6 cases), yolk sac tumor ( 4 cases), seminoma (3 cases), teratoma (2 cases), and embryonal carcinoma ( 1 case).

Conclusions: Less than half of cases have the same stage and histological subtype. There is a tendency to overstage from outside institutions. Yolk sac tumor was the most under-recognized histologic subtype, while choriocarcinoma was the most often overdiagnosed. Pathologists evaluating orchiectomy specimens should be aware of the major pitfalls in classification and staging, many of which may have impact on patient management.

942 Needle Biopsy of Renal Masses: Analysis of 121 Matched Biopsy and Resection Cases

Saul E Harari, Daniel J Sassoon, John Eble, Liang Cheng, Mohammed Idrees, Helen E Michael, David J Grignon. Indiana University School of Medicine, Indianapolis, IN. Background: Performance of needle biopsy in the setting of renal mass is increasingly common and presents pathologists with new challenges in diagnosing, subtyping and grading specimens.

Design: A database search was conducted to identify patients with in-house renal biopsy and corresponding resection specimens. Starting from 2008, 121 such patients were identified for analysis.

Results: The study included 121 biopsies of renal masses with accompanying resection specimens. The biopsy diagnoses included 97 renal cell carcinomas $(80 \%), 7$ nonneoplastic/non-diagnostic $(6 \%), 3$ renal epithelial neoplasms not further classified $(2 \%), 3$ urothelial carcinomas ( $2 \%), 3$ nephroblastomas ( $2 \%), 2$ oncocytomas $(2 \%)$, and 6 miscellaneous (5\%).

The diagnosis of renal cell carcinoma was confirmed in 96/97 cases (99\%). 83 biopsy diagnoses ( $86 \%$ ) of renal cell carcinoma were also definitively subtyped . Of these, 76 $(92 \%)$ were confirmed at resection. The discrepant cases included 4 clear cell renal cell carcinomas later deemed unclassifiable, 1 clear cell renal cell carcinoma changed to Xp11 translocation renal cell carcinoma, 1 mucinous tubular and spindle cell carcinoma to type 1 papillary renal cell carcinoma, and 1 type 1 papillary renal cell carcinoma to type 2 papillary renal cell carcinoma.

A Fuhrman nuclear grade was assigned in 71 of the biopsies diagnosed as clear cell or papillary renal cell carcinoma $(72 \%)$. The grade upon resection was the same in 45 cases $(63 \%)$, and higher in $26(37 \%)$. There were no instances of downgrading.

Of the 7 biopsies with non-neoplastic diagnoses, the resection specimen revealed carcinoma in 6 cases $(86 \%)$. The 7 th was diagnosed at biopsy as fibrotic tissue with chronic inflammation, which was confirmed as pyelonephritis at resection.

Significant diagnostic discrepancies included one case changed from sarcomatoid carcinoma to dedifferentiated liposarcoma and one from renal cell carcinoma to a melanocytic neoplasm.

Conclusions: Needle biopsy of renal masses can reliably diagnose the mass given that lesional tissue is obtained. Subtyping of renal cell carcinoma is possible in the majority of cases with a high degree of accuracy. Nuclear grading on biopsy reliably provides a minimum grade, but is limited by a significant frequency of under-grading.

943 Prostate Cancer Grade Group 4: Gleason $3+5=8$ and $5+3=8$ Outcomes Versus $4+4=8$

Nicholas Harding-Jackson, Elizabeth Whittington, Daniel C Eastwood, George A Tjionas, Oleksandr N Kryvenko, Swikrity Upadhyay, Merce Jorda, Kenneth A Iczkowski. Medical College of Wisconsin, Milwaukee, WI; University of Miami, Miami, FL.

Background: 5 prognostic grade groups (G $1-5$ ) are in use for prostatic biopsies (PMID:23464824): Gleason scores (GS) $6,3+4=7,4+3=7,4+4=8$, and 9-10. Regarding G4, outcome in less-prevalent, GS $3+5$ and $5+3=8$ tumor vs. $4+4=8$ is uncertain. Design: Biopsy slide sets accessioned from 2003-13 at 2 institutions, with highest GS (in $\geq 1$ part) of $3+5=8$ or $5+3=8$, were regraded by urologic pathologists based on ISUP 2014 criteria. Contemporaneous controls with highest GS 4+3, 4+4, or $4+5$ were reviewed. Follow-up was based on electronic medical record.

Results: Men with recurrent, persistent, or metastatic cancer were $24.1 \%$ in GS $4+3$, $67.3 \%$ in GS $4+4,77.5 \%$ in GS $3+5,73.4 \%$ in GS $5+3$, and $74.3 \%$ in GS $4+5$. Death from cancer was significant $(\mathrm{p}=.002)$, with $31 \% 36$-month incidence in GS $5+3$, versus $16 \%$ in GS $4+5$ and $3.1 \%$ in GS $3+5$. Biopsy GS did not differ in prostatectomy findings: grade, stage, or margin status; men with biopsy GS $4+4$ had mean $28 \%$ gland involved vs. $27 \%$ for GS $3+5 / 5+3$.

\begin{tabular}{|l|l|l|l|l|l|l|}
\hline Biopsy GS--> & $\mathbf{4 + 3}$ & $\mathbf{4 + 4}$ & $\mathbf{3 + 5}$ & $\mathbf{5 + 3}$ & $\mathbf{4 + 5}$ & $\mathbf{p}$ \\
\hline $\mathrm{n}=$ & 56 & 113 & 54 & 17 & 112 & \\
\hline $\begin{array}{l}\text { Follow-up mos. mean/ } \\
\text { median }\end{array}$ & $45 / 46$ & $45 / 35$ & $47 / 43$ & $14 / 7$ & $33 / 26$ & $<0.001^{1}$ \\
\hline $\begin{array}{l}\mathbf{1}^{\circ} \text { Therapy: } \dagger \\
\text { Prostatectomy }\end{array}$ & $26.8 \%$ & $29.5 \%$ & $25 \%$ & $13.3 \%$ & $12.1 \%$ & $0.005^{2}$ \\
\hline ADT and/or XRT & $48.2 \%$ & $64.2 \%$ & $66.7 \%$ & $73.3 \%$ & $74.6 \%$ & \\
\hline Cryotherapy & $12.5 \%$ & $3.2 \%$ & $2.1 \%$ & $6.7 \%$ & $2.0 \%$ & \\
\hline Manage expectantly & $12.5 \%$ & $3.2 \%$ & $6.3 \%$ & $6.7 \%$ & $11.1 \%$ & \\
\hline Outcome: $\dagger$ BCR & $11.1 \%$ & $6.8 \%$ & $10.2 \%$ & $6.7 \%$ & $3.0 \%$ & $<0.001^{2}$ \\
\hline Persistent cancer* & $7.4 \%$ & $28.2 \%$ & $40.8 \%$ & $40.0 \%$ & $21.8 \%$ & \\
\hline Metastatic cancer & $5.6 \%$ & $22.3 \%$ & $26.5 \%$ & $26.7 \%$ & $49.5 \%$ & \\
\hline No evidence of tumor & $75.9 \%$ & $42.7 \%$ & $22.5 \%$ & $26.7 \%$ & $25.7 \%$ & \\
\hline $\begin{array}{l}\text { Death from cancer } \\
\text { (36-month Cumulative } \\
\text { Incidence) }\end{array}$ & $2 / 54(0)$ & $\begin{array}{l}7 / 106 \\
(0.034)\end{array}$ & $\begin{array}{l}2 / 46 \\
(0.024)\end{array}$ & $\begin{array}{l}2 / 14 \\
(0.314)\end{array}$ & $\begin{array}{l}15 / 100 \\
(0.162)\end{array}$ & $0.002^{345}$ \\
\hline
\end{tabular}

${ }^{1}$ Kruskal-Wallis Test ${ }^{2}$ Exact Chi-Square test ${ }^{3}$ Gray's Test ${ }^{4} \mathrm{p}=0.023$ with $4+5$ excluded ${ }^{5} \mathrm{p}=0.011$ for $4+4$ and $3+5$ combined vs. $5+3$. $\dagger$ If known. ADT/XRT: androgen deprivation, radiotherapy. BCR: Biochemical recurrence= serum PSA post-op. rise to $0.2 \mathrm{ng} / \mathrm{mL}$. *Proven cancer or PSA progression after ADT/XRT, or cryotherapy. Conclusions: Current data suggest that GS $3+5 / 5+3$ cancers on biopsy are at least as aggressive as $4+4$. Men with $3+5 / 5+3$ were slightly less likely to undergo prostatectomy. The worse disease-free and death rates of GS $5+3$ in particular, would support its placement in Group 5, which is proven to carry worse outcome than Group 4 (PMIDs:25623747, 24129893).

944 Active Surveillance for Newly Diagnosed Low Risk Prostate Cancer: Institutional Experience of Initial Choice and Follow Up in a Racially Mixed Population

Oudai Hassan, MHD Fayez Daaboul, Dongping Shi, Isaac Powell, Michael L Cher, Wael Sakr. WSU, Detroit, MI.

Background: More patients diagnosed with low risk prostate cancer ( $\mathrm{PCa})$ are choosing active surveillance (AS) for initial management of their disease. In addition to variations in clinicopathological criteria, follow up designs and definition of progression, the representation of minorities in reported AS series is limited. This objective of this study is to reports our institutional experience with AS and follow up biopsy (Bx) findings in a racially mixed population.

Design: All patients who opted for AS between 2008-2015 at our institution were included. Ethnicity was classified as African American (AA), European American (EA) or others. Detailed pathological findings of initial and follow up Bx were documented. Progression was defined as higher Gleason score (GS), more cores with PCa or higher $\%$ core involvement on follow up Bx. Opposite criteria were used to define tumor "downgrading".

Results: One hundred and twenty two patients, 66 (54\%) AA, 25 (21\%) EA and 31 $(25 \%)$ of other ethnicites were included. 104 patients $(85 \%)$ had $\mathrm{PCa}$ in $<=3$ cores, $<=50 \%$ involvement of any core and GS of $<=6$. Eleven patients $(9 \%)$ had a component of Gleason pattern 4 on initial $\mathrm{Bx}$ and $7(6 \%)$ had $>3$ positive cores and/or $>50 \%$ involvement of any positive core. Median follow up was 14 months (1-51). More EA patients had"down graded" PCa on follow up bx and less discrepancy in GS between bx and RP. There were no statistically significant differences among the ethnic groups analyzed in disease progression or the parameters summarized in (Table 1).

\begin{tabular}{|l|l|l|l|l|}
\hline Study Groups / Subsets & Overall & AA & EA & Others \\
\hline Patients on AS & 122 & $66(54 \%)$ & $25(21 \%)$ & $31(25 \%)$ \\
\hline Median \# of follow up Bxs & $1(1-3)$ & $1(1-3)$ & $1(1-3)$ & $1(1-3)$ \\
\hline Progression & $35(29 \%)$ & $19(29 \%)$ & $7(28 \%)$ & $9(29 \%)$ \\
\hline Downgraded & $10(8 \%)$ & $5(8 \%)$ & $4(16 \%)$ & $1(3 \%)$ \\
\hline Same findings & $47(39 \%)$ & $25(37 \%)$ & $7(28 \%)$ & $15(48 \%)$ \\
\hline Negative follow up Bxs & $30(24 \%)$ & $17(26 \%)$ & $7(28 \%)$ & $6(20 \%)$ \\
\hline Opted for RP & $17(14 \%)$ & $8(12 \%)$ & $4(16 \%)$ & $5(16 \%)$ \\
\hline Higher GS on RP & $6(35 \%)$ & $3(38 \%)$ & $1(25 \%)$ & $2(40 \%)$ \\
\hline
\end{tabular}

Conclusions: The majority of men who chose AS for initial management of low risk PCa show same or lower pathology on follow up Bxs. We have not found ethnically based significant differences in disease progression in our cohort. These preliminary results vary from previous reports possibly due to number analyzed and the relatively shorter follow up. Larger, multi institutional studies applying similar inclusion criteria to ethnically mixed populations are needed for more definitive results. 
945 Genitourinary Rhabdomyosarcoma - A Comparative Analysis of Adult vs. Pediatric Cases

Jing He, Hernan Correa, Justin M Cates, Giovanna A Giannico, Omar Hameed, Lan L Gellert. Vanderbilt University, Nashville, TN.

Background: The majority of genitourinary rhabdomyosarcomas (GU-RMS) occur in patients aged $<15$ years. Adult onset GU-RMS is very rare and there is limited data in the literature. The goal of this study was to perform a comparative analysis of adult vs. pediatric GU-RMS.

Design: A retrospective search of the pathology database from 1995 to 2015 was performed to identify all cases of GU-RMS diagnosed at our institution. Pathological features were reviewed and clinical data were collected from the charts.

Results: Overall, 31 GU-RMS cases were identified (median age: $8 \mathrm{y} ; \mathrm{M} / \mathrm{F}$ ratio: 25/6), including 8 adults and 23 children. Clinical and pathologic variables are summarized in table 1 .

The most notable differences between adult and pediatric cases include tumor location, histologic type and stage at presentation. The most common location in adult cases was prostate $(4 / 8,50 \%)$, while majority of pediatric cases occurred in either bladder $(11 / 23$, $48 \%)$ or testis/paratesticular region $(10 / 23,43 \%, \mathrm{p}=0.03) .3$ of the $8(37.5 \%)$ adult cases were alveolar RMS (ARMS), in comparison to 1 of $23(4.3 \%)$ pediatric cases $(\mathrm{p}=0.10)$. The diagnosis of ARMS is predominantly based on histology, with 2 of 3 adult ARMS positive for $\mathrm{t}(2,13)$ or $\mathrm{t}(1,13)$ fusion transcripts on molecular tests. $75 \%(6 / 8)$ of adult cases presented at stage III or higher, compared to $35 \%(8 / 23)$ in pediatric cases $(\mathrm{p}=0.10)$. Outcome information is available for 24 cases with median follow up time of 8 years. 3 adult cases (1 ARMS, 2 ERMS) died of disease with median survival of 20 months. 2 of 4 pediatric cases died of disease at 1 and 4 years. The other 2 pediatric cases died of secondary Ewing's sarcoma and synovial sarcoma at 6 and 12 years.

\begin{tabular}{|l|l|l|l|l|l|}
\hline \multicolumn{1}{|c|}{ Variable } & & Total & Adult & Children & P value \\
\hline Overall & & 31 & 8 & 23 & \\
\hline Age(median,yrs) & & & 20 & 5 & \\
\hline Gender & & & & & 0.57 \\
\hline & Male & 25 & 7 & 18 & \\
\hline & Female & 6 & 1 & 5 & \\
\hline Tumor location & & & & & 0.03 \\
\hline & Prostate & 6 & 4 & 2 & \\
\hline & Bladder & 12 & 1 & 11 & \\
\hline & Testis/Paratesticular & 13 & 3 & 10 & \\
\hline RMS histologic type & & & & & 0.10 \\
\hline & Embryonal & 25 & 5 & 20 & \\
\hline & Alveolar & 4 & 3 & 1 & \\
\hline & Other & 2 & 0 & 2 & \\
\hline Stage & & & & & 0.10 \\
\hline & Stage I and II & 17 & 2 & 15 & \\
\hline & Stage III and IV & 14 & 6 & 8 & \\
\hline Overall survival (n=24) & & & & & 0.37 \\
\hline & Alive & 17 & 4 & 13 & \\
\hline & Dead & 7 & 3 & 4 & \\
\hline
\end{tabular}

Conclusions: Compared to pediatric cases, adult onset GU-RMS more often involve the prostate gland, exhibit alveolar histology, and present at higher stage.

946 Mucinous Adenocarcinoma of Prostate and Prostatic Adenocarcinoma with Mucinous Components; A Clinicopathological Analysis of 81 Cases

Samaratunga Hemamali, Brett Delahunt, Lars Egevad, John Yaxley, Shulammite Johannsen, Geoff Coughlin, Troy Gianduzzo. Aquesta Pathology, Brisbane, Queensland, Australia; University of Queensland, Brisbane, Queensland, Australia; Wellington School of Medicine and Health Sciences, Wellington, Otago, New Zealand; Karolinska Institute, Stockholm, Sweden; Wesley Hospital, Brisbane, Queensland, Australia. Background: The clinical significance of mucinous components in prostatic adenocarcinoma remains uncertain.

Design: The Aquesta Pathology database revealed 81 cases of prostatic adenocarcinoma with mucinous components in radical prostatectomy specimens since 2008. These were reviewed and graded using the 2014 International Society of Urological Pathology (ISUP) recommendation of grading the underlying architecture. We present clinicopathological characteristics of this group and compare with those of a Gleason score (GS) matched group with no mucinous components.

Results: Mucinous components of $5-100 \%$ were found in $2.4 \%$ and $\geq 25 \%$ in $35(1.0 \%)$ of 3375 cases. The mean age was 61.8 years (range 44-74), the mean preoperative serum PSA $8.65 \mathrm{ng} / \mathrm{mL}$ (range 2.5- 25.2) and clinical stage cT1 in $81 \%$ and cT2 in 19 $\%$. The morphology of the mucinous component included cribriform glands in $83 \%$, single well-formed glands in $91 \%$ and poorly formed glands and fused glands each in $100 \%$ of cases. Papillary structures were seen in one cancer and single cells/strands of cells in another. In $15 \%$ of cases with extraprostatic extension, mucinous components were found outside the capsule. Gleason scores were $3+4$ in $13.6 \%, 4+3$ in $53.1 \%$, $4+4$ in $2.5 \%, 3+4$ or $4+3$ with tertiary 5 in $12.3 \%$ and $9-10$ in $18.5 \%$. Extraprostatic extension (EPE) was found in $50.6 \%$ of cases ( $44.4 \%$ established EPE). In $24.6 \%$, the tumour volume was $\geq 3 \mathrm{~cm}^{3}$ and $11 \%$ had surgical margin positivity. Seminal vesicle involvement was found in $3.8 \%$ and 1 of 23 cases who had a lymphadenectomy had lymph node metastases $(4.3 \%)$. Comparatively, grade-matched non-mucinous carcinoma had EPE, tumour volume of $\geq 3 \mathrm{~cm}^{3}$, surgical margin positivity, seminal vesicle involvement and lymph node metastases in $38.3 \%$ (34.5\% established), $38 \%$, $12.3 \%, 6.2 \%$ and $3.2 \%$ respectively. The 5 year biochemichal recurrence-free survival rates for mucinous vs. non-mucinous cancer were $18.6 \%$ vs. $17.6 \%$

Conclusions: Prostatic adenocarcinoma with mucinous components is often high grade with $86 \%$ of cases having a GS of $>3+4$. However, the prognosis of this group appears to be similar to non-mucinous cancers of similar GS

947 Concurrent TMPRSS2-ERG and SLC45A3-ERG Rearrangements Plus PTEN Loss Are Associated with an Aggressive Tumor Subset of Prostate Cancer

Silvia Hernandez, Alba Font-Tello, Nuria Juanpere, Silvia de Muga, Marta Lorenzo, Lluis Fumado, Sergio Serrano, Josep Lloreta. Universitat Pompeu Fabra, Barcelona, Spain; Hospital del Mar-Parc de Salut Mar-IMIM, Barcelona, Spain; Autonomous University of Barcelona, Barcelona, Spain.

Background: $S L C 45 A 3$ is the second most common 5' partner gene among the $E R G$ rearrangements in PrCa. Some papers have addressed the potential role of the SLC45A3$E R G$ rearrangement in prostate cancer. TMPRSS2 and SLC45A3 rearrangements have been found to coexist in the same tumor in a subset of cases, but the meaning of this association is still unknown.

Design: SLC45A3-ERG and TMPRSS2-ERG rearrangements and their association with $E R G$ and $P T E N$ expression and with pathological features have been analyzed in a series of $80 \mathrm{PrCa}$ (PSMAR-Biobank, Barcelona, Spain). ERG and PTEN mRNA expression were assessed by quantitative RT-PCR; both TMPRSS2-ERG and SLC45A3-ERG were assessed by RT-PCR and FISH. SLC45A3-ERG and TMPRSS2-ERG rearrangements were also sequenced; and ERG protein expression was analyzed by IHC. Pearson ChiSquare or Fisher's Exact test were used for categorical variables, and Kaplan-Meier for PSA progression-free survival.

Results: TMPRSS2-ERG was the only rearrangement in $51.6 \% \mathrm{GS} \leq 7$ and $22.2 \% \mathrm{GS}>8$ tumors ( $\mathrm{p}=0.027)$. SLC45A3-ERG was found in $25(31.25 \%)$ cases, 20 of them with concurrent TMPRSS2-ERG rearrangement: $11.5 \% \mathrm{GS}=6,22.2 \% \mathrm{GS}=7$, and $50 \% \mathrm{GS} \geq 8$ tumors ( $\mathrm{p}=0.013)$.Double rearrangements were associated with higher levels of $E R G$ mRNA ( $\mathrm{p}=0.04)$. Double rearrangement plus PTEN loss was detected in $0 \% \mathrm{GS}=6$; $14.7 \% \mathrm{GS}=7$, and $29.4 \% \mathrm{GS}>8$ tumors $(\mathrm{p}=0.032$ ). Furthermore, this triple change was present in $19.2 \%$ stage T3-4 but not in any of the stage T2 tumors $(\mathrm{p}=0.05)$. There was a high correlation between detection of both rearrangements by FISH and RT-PCR (TMPRSS2-ERG $\mathrm{\kappa}=0,922 ;$ SLC45A3-ERG $\mathrm{\kappa}=0,866$ ). No relationship was found with PSA progression-free survival.

Conclusions: Single TMPRSS2-ERG fusion is associated with low grade $\mathrm{PrCa}$. Subsequent development of SLC45A3-ERG results in higher ERG expression. TMPRSS2-ERG/SLC45A3-ERG rearrangements are associated with high grade PrCa. The combination of double rearrangement and PTEN loss, according to our series, is never found in low grade, low stage tumors. These findings could be potentially useful in therapeutic decision making in $\mathrm{PrCa}$.

Supported by FIS/ Instituto Carlos III/FEDER/PI12/01426, Spanish Ministry of Health.

948 FOXO1 and p53 Are Associated with Poor Prognosis in Urothelial Cell Carcinoma

Silvia Hernandez, Alba Font-Tello, Nuria Juanpere, Marta Lorenzo, Lara Nonell, Albert Frances, Sergio Serrano, Josep Lloreta. Universitat Pompeu Fabra, Barcelona, Spain; Hospital del Mar-Parc de Salut Mar-IMIM, Barcelona, Spain; Parc de Salut Mar-IMIM, Barcelona, Spain; Hospital del Mar-Parc de Salut Mar-IMIM, Barcelona, Spain; Autonomous University of Barcelona, Barcelona, Spain.

Background: Nuclear FOXOs stop cell cycle progression, promote apoptosis, and negatively control angiogenesis. They are downregulated in various cancer types. In spite of extensive literature, the prognostic value of $\mathrm{p} 53$ in urothelial carcinoma remains unclear in advanced disease and high grade superficial tumors. FOXOs and $\mathrm{p} 53$ could play similar roles as tumor suppressor genes. However, little is known about the role of FOXO1 and its relationship with the $\mathrm{p} 53$ status in bladder cancer.

Design: FOXO1 and p53 expression were analyzed by immunohistochemistry (IHC) in 162 and 155 urothelial cell carcinomas, respectively. Tumor samples were selected retrospectively from Parc de Salut MAR Biobank (MARBiobanc) of Barcelona, Spain. IHC was performed in FFPE TMA sections. FOXO1 and p53 histoscores were obtained. Results: Decreased FOXO1 expression (histoscore values $<10$ ) was associated with high grade, WHO $2004(\mathrm{p}=0.030)$; grade 3 , WHO $1973(\mathrm{p}=0.025)$; high stage $(\mathrm{p}=0.0001)$, and high stage/grade bladder tumors $(\mathrm{p}=0.0004)$. $\mathrm{p} 53$ overexpression (histoscore values $>20$ ) was associated with high grade, WHO $2004(\mathrm{p}=0.017$ ); grade 3, WHO $1973(\mathrm{p}<0.0001)$, high stage $(\mathrm{p}<0.0001)$ and high stage/grade $(\mathrm{p}<0.0001)$. Comparing $w t$ cases $v s$ to those harboring one or the two alterations, the combination FOXO1 downregulation/p53 overexpression is strongly associated with high grade, WHO $2004(\mathrm{p}=0.004)$; grade 3 , WHO $1973(\mathrm{p}<0.0001)$, high stage $(\mathrm{p}<0.0001)$, and high stage/grade $(\mathrm{p}<0.0001)$ bladder tumors. $\mathrm{p} 53$ overexpression was associated with tumor progression ( $\log$ Rank, $\mathrm{p}=0.01$ ), while decreased FOXO1 showed a trend to be associated with it ( $\log$ Rank, $\mathrm{p}=0.06$ ). To harbor one alteration (FOXO1 downregulation or p53 overexpression), was even more strongly associated with bladder tumor progression (Log Rank, $\mathrm{p}=0.005)$.

Conclusions: Decreased FOXO1 expression and $\mathrm{p} 53$ overexpression are associated with grade, stage and progression. This combination of prognostic markers could be helpful in the prognostic stratification of bladder cancer patients.

Supported by FIS/ Instituto Carlos III/ FEDER $<$ ins cite="mailto:E0037" datetime="2011-02-28T09:50">/</ins $>$ PI12/01426, Spanish Ministry of Health. 
949 Atypical Cribriform Lesion of the Prostate Shares Similar ERG and PTEN Expression Patterns as Intraductal Carcinoma of the Prostate and Is Associated with a Higher Stage and Grade Than Invasive Cancer Alone Richard Hickman, Hui Yu, Max Kong, Rajal Shah, Ming Zhou, Jonathan Melamed, Fang-Ming Deng. NYU Langone Medical Center, New York, NY; Miraca Life Sciences, Dallas, TX.

Background: Atypical cribriform lesion (ACL) of the prostate exhibits greater architectural complexity and/or nuclear atypia than high grade prostate intraepithelial neoplasia (HGPIN), but lacks the diagnostic features for intraductal carcinoma (IDC). The clinicopathological significance of ACL is unclear. Since IDC is associated with nuclear ERG expression and cytoplasmic PTEN loss, whereas HGPIN is usually not, we sought to investigate the ERG and PTEN status of ACL and its topographic relationship with invasive acinar carcinoma (IAC). We also compared the clinical characteristics of ACL- and IDC-associated carcinoma with cases of IAC without ACL/ IDC.

Design: We examined 310 radical prostatectomy specimens that were received within our institution between July 2013 and December 2014 using hematoxylin and eosin staining with adjunct PIN4 immunohistochemistry. Lesions were classified as ACL if 1) they displayed a loose cribriform arrangement wherein $>50 \%$ of the intraductal mass consisted of punched-out luminal spaces, and 2) they showed relatively uniform nuclei that lack the classic nuclear features of IDC. ERG and PTEN immunohistochemistry was conducted on blocks with the most prominent cribriform lesions from each prostate $(\mathrm{n}=46, \mathrm{n}=34$, respectively).

Results: 46 cases had either ACL ( $\mathrm{n}=10)$, IDC $(\mathrm{n}=6)$ or combined ACL and IDC $(\mathrm{n}=30)$. All ACL and IDC foci, except 1 ACL focus, were located within $3 \mathrm{~mm}$ of IAC. The ERG status of all ACL and IDC cases were identical to the nearby IAC, contrasting with $50 \%$ of HGPIN $(p<0.01)$. The frequency of uniform PTEN loss in studied 34 cases was similar in ACL and IDC (66.7\%; 66.6\%, respectively). Most cases with ACL and IDC had concordant PTEN expression patterns as the nearby IAC $(66.7 \%, 76.6 \%$, respectively). No cases of HGPIN showed uniform PTEN $\operatorname{loss}(0 \%, \mathrm{p}<0.01)$. ACL- and or IDC-associated carcinoma $(\mathrm{n}=46)$ had a higher stage (stage 3$)$ and grade (Gleason score: $4+3=7$ or greater) compared to IAC-only $(n=264, p<0.01)$.

Conclusions: ACL and IDC are in proximity to IAC, share similar ERG and PTEN expression profiles and are associated with a higher stage and grade than IAC-only. Despite ACL appearing less worrisome than IDC, the finding of ACL in biopsy or prostatectomy specimens warrants prompt attention and management.

950 Do Specific Gleason Pattern 4 Morphologies Detected on Transrectal Ultrasound (TRUS)-Guided Biopsies Predict Upgrading or Upstaging in Gleason Score 3+4=7 Prostate Cancer?

Kevin Hogan, Susan J Robertson, Nicola Schieda, Kien T Mai, Daniel T Keefe, Eric C Belanger, Rodney H Breau, Chris Morash, Trevor A Flood. The Ottawa Hospital, Ottawa, ON, Canada; The University of Ottawa, Ottawa, ON, Canada.

Background: Selected patients with Gleason score (GS) $3+4=7$ prostate cancer (PCa) detected on TRUS-guided biopsies may be considered for active surveillance (AS); however, a proportion of these will harbor more aggressive disease. The purpose of this study was to determine if specific morphologies of Pattern 4 may predict upgrading and/or upstaging after radical prostatectomy (RP).

Design: A database search for men with GS $3+4=7$ PCa diagnosed on TRUS-guided biopsy that underwent RP between January 2010 and June 2015 identified 154 patients. Two blinded genitourinary pathologists independently reviewed the biopsies and assessed for: ill-defined glands (IDG), fused glands, cribriform formations, and glomerulations. Patient age and serum prostate-specific antigen (PSA) were also recorded. Gleason score and stage (presence or absence of organ-confined disease $[\mathrm{OCD}]$ ) were retrieved from RP reports. Data were compared using multi-variate regression, chi-square and Spearman correlation. Inter-observer agreement was calculated using the Kappa statistic.

Results: The mean patient age was $63.2 \pm 6.5$ years with $28.6 \%$ (44/154) upgraded and $44.8 \%(69 / 154)$ with non-OCD after RP. PSA was associated with both upgrading $(8.5 \pm 5.4$ vs. $6.9 \pm 3.2 \mathrm{ng} / \mathrm{mL},[\mathrm{p}=0.04])$ and non-OCD $(8.2 \pm 4.6$ vs. $6.7 \pm 3.2 \mathrm{ng} /$ $\mathrm{mL},[\mathrm{p}=0.03])$. IDG and fused glands were not associated with upgrading or nonOCD ( $p>0.05)$ with moderate to strong inter-observer agreement $(K=0.61-0.92)$. Cribriform formations were associated with both upgrading and non-OCD $(\mathrm{p}=0.004$, 0.003 respectively) with moderate agreement $(\mathrm{K}=.72)$. Only rare biopsies demonstrated glomerulations and therefore this morphology could not be further analysed.

Conclusions: Presence of cribriform morphology on TRUS-guided biopsy is predictive of upgrading and upstaging at RP whereas IDG and fused glands were not useful. Cribriform morphology may be of importance when considering patients with GS $3+4=7$ PCa for active surveillance.

951 Oncocytic Adrenal Cortical Neoplasm, a Clinical and Pathological Study of 39 Cases

He Huang, Priya Rao, Kanishka Sirca, Mouhammed A Habra, Miao Zhang. The University of Texas MD Anderson Cancer Center, Houston, TX.

Background: Oncocytic adrenal cortical neoplasm (OACN) is rare. Proper categorization remains a challenge since the Weiss criteria for classifying conventional adrenal cortical neoplasms (CACN), is not entirely applicable to OACN. Quantitative Ki-67 (qKi-67), using clinically to select patients for chemotherapy, has not been evaluated in $\mathrm{OACN}$ patients.

Design: We identified 39 cases of OACN from 1990-2015. Tumors had been categorized as benign, uncertain malignant potential (UMP) and carcinoma. qKi-67 was performed on 22 cases. CACN with comparable Ki-67 were evaluated as controls.

Results: The cohort includes 23 female and 16 male OACN patients (mean age: 47.2 yr). OACN were categorized by pathologic features (necrosis, mitotic activity, capsular or venous invasion and metastasis) into benign $(\mathrm{n}=8), \mathrm{UMP}(\mathrm{n}=22)$ and carcinoma $(\mathrm{n}=9)$. All benign tumors were absent of these features. All carcinomas exhibited at least two of these features. UMP cases had atypical features, such as large tumor size, diffuse growth pattern and/or marked nuclear atypia. qKi-67 was performed on 22 cases (4 benign (mean: 4\%), 13 UMP (mean: $9 \%$ ) and 5 carcinomas (mean: 12\%)). Follow up information was available for 17 cases. 3 patients with benign tumors had no evidence of disease (NED) (mean follow up (f/u): 4.7 m). 9 UMP patients had 8 NED and one death of unknown cause (mean f/u: $13.1 \mathrm{~m}$ ). Among 5 patients with carcinoma (mean f/u: $23.6 \mathrm{~m}$ ), 1 had NED, 3 were alive with disease (AWD) and 1 died of disease (DOD). The control cohort consisted of $20 \mathrm{CACN}$ with comparable clinical features. All 3 patients with adenoma (mean f/u: $16.7 \mathrm{~m}$; mean Ki-67 (mKi-67): $3 \%$ ) and 6 patients with UMP (mean f/u: $21.6 \mathrm{~m} ; \mathrm{mKi}-67: 6 \%$ ) had NED. Of the 11 patients with carcinoma (mean f/u: 35.3 m; mKi-67: 18\%), 4 had NED, 3 were AWD and 4 were DOD. High qKi-67 correlated with clinical outcome in CACN where it was 9\% in NED vs $19 \%$ in non-NED (AWD+DOD) patients ( $p=0.01, \mathrm{f} / \mathrm{u} 28.3 \mathrm{~m}$ ). However, qKi-67 did not correlated with outcome in OACN patients $(p=0.9, \mathrm{f} / \mathrm{u} 14.7 \mathrm{~m})$.

Conclusions: We examined the largest cohort of $\mathrm{OACN}$ cases and found that benign and UMP tumors behaved comparably to $\mathrm{CACN}$ while oncocytic carcinoma appeared less aggressive than the conventional counterparts that had similar qKi-67. qKi-67, an objective measurement for clinical decision making for additional toxic chemotherapy, is applicable in CACN. However, it is not as effective in separating aggressive from indolent $\mathrm{OACN}$.

\section{Proliferation Index of Gleason Grade 3 Prostate Adenocarcinoma} with and without Associated Grade 4

Michael Hwang, Liying Fu, Adebowale Adeniran, Peter A Humphrey. Yale University School of Medicine, New Haven, CT.

Background: Gleason score (GS) 6 cancer in the most recently modified Gleason grading scheme represents a more uniform group with a more indolent behavior. It is unclear if Gleason grade 3 in pure form is different from Gleason grade 3 associated with grade 4 cancer. Ki67 labeling or proliferation index (Ki67 LI) is a promising prognostic factor with higher values associated with disease aggressiveness. This study aimed to establish whether Ki67 LI is different in Gleason grade 3 (G3) component in GS 6 cancer compared to Gleason grade 3 in cancer with GS $\geq 7$.

Design: The pathology database was searched for prostatectomy specimens with a diagnosis of prostatic adenocarcinoma with GS 6 and GS 7 to 9 (2009 to 2013). The 2011 AFIP modified Gleason scheme was used in assigning Gleason scores. Ki67 immunostains were performed and were divided into three groups for analysis: GS 6 , and GS $\geq 7$ with or without cribriform pattern. The Ki67 LI was defined as the number of immunoreactive cells divided by the total counted cells on printed color images taken from areas with highest Ki67 positivity. Non-paired t test and Pearson's correlation test were used for statistical analysis.

Results: 49 cases of GS $\geq 7$ and 18 cases of GS 6 were selected. The Ki67 LI of Gleason grade 3 was $2.33 \%$ in GS 6 cases ( $\mathrm{n}=18), 2.73 \%$ in GS $\geq 7$ cases without cribriform pattern ( $\mathrm{n}=14)$, and $3.78 \%$ in $\mathrm{GS} \geq 7$ cases with cribriform pattern ( $\mathrm{n}=35)$. A pair-wise analysis showed positive correlation of Ki67 LI between grade 3 and grade 4 in cases with cribriform pattern ( $\mathrm{n}=35$; Pearson's $\mathrm{r}=0.432 ; \mathrm{p}=0.01)$ and no correlation between grade 3 and grade 4 in cases without cribriform pattern $(n=14$; Pearson's $r=-0.102$; $\mathrm{p}=0.728$ ). The Ki67 LI of grade 3 associated with cribriform pattern was higher than pure grade $3(\mathrm{p}=0.039)$.

Conclusions: The higher proliferation index of Gleason grade 3 associated with cribriform grade 4 compared to pure grade 3 suggests that not all Gleason grade 3 carcinoma cells are identical. Gleason grade 3 cancer may be influenced by associated high-grade cancer, especially cribriform adenocarcinoma.

\section{PTEN Expression in Chromophobe Renal Cell Carcinoma Versus} Clear Cell Renal Cell Carcinoma

Gena Ibrahim, Manal Gabril, Christopher Howlett. Western University/ London Health Sciences Centre, London, ON, Canada.

Background: Chromophobe renal cell carcinoma (chRCC) is a rare type of renal tumors. Less is known in literature regarding the genetic mutations of chRCC. PTEN genes are tumor suppressor genes that normally regulate cell growth and apoptosis. Some of the studies confirmed that PTEN inactivation might contribute to renal cell carcinoma. Loss-of-function mutations of PTEN, a negative regulator of m-TOR through the PI3K/Akt pathway, are found in approximately $5 \%$ of patients with RCC and this genetic mutations that lead to constitutive increase in m-TOR activity. Our objectives of the study were to determine the status of PTEN protein expression and subsequently activation (positive expression) of m-TOR and Phospho-4E-BP1 markers in chRCC vs. clear cell renal cell carcinoma (ccRCC).

Design: We built a tissue microarray (TMA) containing duplicate cores from 84 renal tumors specimens ( $35 \mathrm{chRCC}$ and $49 \mathrm{ccRCC}$ cases) performed at LHSC were included (2000-2010). Then we investigated the immunohistochemistry expression of the PTEN, Phosho-mTOR and Phospho-4E-BP1 in TMA. Immunoexpression of three markers were evaluated and semi-quantitatively classified as absent/ loss, weak, moderate, or strong and assessed for both cytoplasmic and nuclear expression. Clinicopathological data (tumor grade, tumor stage, survival data and metastasis) was collected. The results of PTEN expression will be confirmed with using Fish analysis of PTEN. Results: 27 out of $35(77.1 \%)$ cases of chRCC and 24 out of 49 (48.9\%) cases of ccRCC showed weak to loss expression of PTEN. 5 out of these 27 cases of chRCC and 19 out of these 24 cases of ccRCC showed positive of either Phosho-mTOR or Phospho4E-BP1 expression (table 1). 17 out of 27 cases $(62.9 \%)$ of chRCC with weak or loss PTEN expression showed high Fuhrman grade 3 or $4 / 4$. 
Table 1: Correlation between PTEN expression and Phospho-mTOR/Phospho4E-BP-1 expression

\begin{tabular}{|c|c|c|}
\hline \multicolumn{2}{|l|}{ PTEN exp } & $\begin{array}{l}\text { Increased Phospho-mTOR or } \\
\text { Phospho-4E-BP1 expression }\end{array}$ \\
\hline \multicolumn{2}{|l|}{$\operatorname{chRCC}(\mathrm{n}=35)$} & \multirow[b]{2}{*}{$5 / 27(18.5 \%)$} \\
\hline $\begin{array}{l}\text { weak PTEN exp } \\
(5 / 35)\end{array}$ & $\begin{array}{l}\text { loss PTEN exp } \\
(22 / 35)\end{array}$ & \\
\hline \multicolumn{2}{|l|}{ ceRCC $(n=49)$} & \multirow[b]{2}{*}{ 19/24 (79.1\%) } \\
\hline $\begin{array}{l}\text { weak PTEN exp } \\
(5 / 49)\end{array}$ & $\begin{array}{l}\text { loss PTEN exp } \\
(19 / 49)\end{array}$ & \\
\hline
\end{tabular}

Conclusions: Our results indicate that PTEN protein loss is common in chRCC cases. However chRCC cases showed low incidence of increase in expression of $\mathrm{m}$-TOR or Phospho-4E-BP1 in contrast to ccRCC cases. These results might help in understanding more about PTEN mutations in chRCC as well as its relationship to m-TOR activation of PI3K/Akt pathway. Also, this will offer some basis for the development of modified forms of therapy for this tumor.

954 Racial Disparity in Prostatic Expression of Pro-inflammatory and Paracrine Factors

Kenneth A Iczkowski, Jim R Lambert, Kathleen C Torkko, Daniel C Eastwood, Robert $S$ Wilson, Girish V Shah, MS Lucia. Medical College of Wisconsin, Milwaukee, WI; University of Colorado, Aurora, CO; University of Louisiana, Monroe, LA.

Background: Growth differentiation factor 15 (GDF-15, PDF, NAG-1) is a divergent TGF- $\beta$ superfamily anti-inflammatory cytokine. We have shown that GDF-15 is generally increased in prostate cancer [PMID:12894347] and bears an inverse relationship to inflammation in benign prostate [PMID:25327758]. Calcitonin (CT) functions within the prostate, as a paracrine/ neuroendocrine promoter of prostate cancer (PC) growth, invasion, and metastasis, and also modulates NF-kappaB and associated pro-inflammatory signaling [PMID:18784182].

Design: 7 Tissue microarrays representing 3-4 evaluable punched cores each of $\mathrm{PC}$ and benign tissue taken from prostatectomy specimens from 91 African-American (AA) and 89 white (W) men, matched for Gleason score (6 and 7, mean 6.47), stage, and margin status, were stained. Staining was done with validated goat polyclonal antibody to GDF-15 or CT, and each core was scored based on \% cells and intensity, as 0 to $3+$. Wilcoxon rank-sum statistical test was used to assess differences by race.

Results: GDF-15 expression in PC was higher in AA than W men ( $\mathrm{p}<0.0001$ ), mean 2.16 vs. 1.41 , median 2 vs. 1.5. In benign epithelium, it was also higher but not significantly $(\mathrm{p}=0.14)$, mean 0.99 vs. 0.91 , median 1 vs. 0.5 .

CT reactivity of PC was borderline-decreased in AA men ( $\mathrm{p}=0.06)$, mean 0.90 vs. 1.14 , median 1 vs. 1 ; but no racial differences in $\mathrm{CT}$ were seen in benign epithelium, mean 0.39 vs. $0.55(\mathrm{p}=0.2)$.

Conclusions: Our finding of higher GDF-15 in AA men despite their tendency toward more frequent prostatic inflammation [PMID:9605645] and different gene variants governing inflammation that are predictive of PC [PMID:19830784], suggests that in PC, GDF-15 bears a different relationship to inflammation than the inverse relationship found in benign prostate [PMID:25327758]. That is, the increased GDF-15 expression in PC could serve to counteract an anti-cancer action of inflammation rather than being pro-tumorigenic per se. The trend toward less CT in PC of AA men, though of borderline significance, is consistent with prior research that had shown AA men to have decreased chromogranin A expression in benign [PMID:15882754] and malignant [PMID:22110216] prostate. Experimentally, neuroendocrine cells exert a paracrine effect that inhibits PC growth [PMID:15368468].

This work is supported by the Department of Defense PCBN, Award No. W81XWH-10-2-0056 and W81XWH-10-2-0046.

955 Clinical Significance of Percentage of Gleason Pattern 4 in Gleason Score 7 Prostate Cancer at Radical Prostatectomy

Mohsin Jamal, Sean R Williamson, Mireya Diaz-Insua, Mani Menon, Hans Stricker, James Peabody, Craig G Rogers, Nilesh S Gupta. Henry Ford Hospital, Detroit, MI. Background: We sought to determine the prognostic value of percentage Gleason pattern 4 in radical prostatectomy specimens.

Design: We selected 400 patients who underwent robotic radical prostatectomy (RRP) between 2010 and 2011 with Gleason score 7. Data collected included pT stage, tumor volume, margin status, angiolymphatic invasion and lymph node involvement and biochemical recurrence (BCR) at 2 years after surgery.

Results: Mean age of the patients was 62 years. We stratified the patients into four quartiles based on percentage of Gleason pattern 4 (GP4). Q1: percent of Gleason 4 $<=20, \mathrm{Q} 2:>20 \%-35 \%, \mathrm{Q} 3:>35 \%-65 \%$, Q4: $>65 \%$. Table 1 shows these quartiles correlated with multiple pathological outcomes.

Table 1. Association of categorical pathology outcomes by quartiles of percentage of RRP Gleason 4

Table 1. Association of categorical pathology outcomes by quartiles of percentage of RRP
\begin{tabular}{|l|c|c|c|c|c|}
\hline \multicolumn{1}{|c|}{ Outcome } & Q1 (n=118) & Q2 $(\mathbf{n}=99)$ & $\mathbf{Q 3}(\mathbf{n}=99)$ & $\mathbf{Q 4}(\mathbf{n}=84)$ & p-value \\
\hline Organ confined & 75.4 & 43.4 & 33.3 & 33.3 & $<0.001$ \\
\hline EPE & 24.6 & 56.6 & 66.7 & 66.7 & $<0.001$ \\
\hline SVI & 0 & 7.1 & 14.1 & 22.6 & $<0.001$ \\
\hline Margin + & 22.9 & 27.3 & 25.3 & 33.3 & 0.153 \\
\hline Lymph node + & 0 & 6.1 & 8.1 & 15.5 & $<0.001$ \\
\hline Angio invasion & 0 & 5.1 & 14.1 & 21.4 & $<0.001$ \\
\hline BCR $^{1}$ & 5.1 & 11.5 & 14.3 & 39.8 & $<0.001$ \\
\hline
\end{tabular}
Numbers represent percent of individuals within the quartile of the distribution of percent RRP GP4 with the particular outcome

p-value based on Mantel-Haenszel statistic. ${ }^{1}$ Six cases did not have BCR information.
Percentage of GP4 was associated with BCR when other pathologic features were accounted for. A percent greater than 65 (i.e. fourth quartile) was significantly different from a percent of 20 or less in providing a greater likelihood for recurrence $\mathrm{OR}=6.53$ $(95 \%$ CI $2.43,17.56)$. The other factors that were also significant were margins, positive lymph nodes and angiolymphatic invasion. These four variables yielded a c-statistic of 0.82 . If percentage of GP4 is considered as a continuous measure, it is also independently related with PSA recurrence within 2 years of surgery. A unit increase in the percent of GP4 increases the likelihood of recurrence by 3 percent, $O R=1.03(1.01,1.04)$.

Conclusions: 1. Gleason 7 prostatic adenocarcinoma represents a heterogenous group of tumors. A subgroup of tumors with Gleason score $3+4=7$ with low percentage of GP4 $(<=20 \%)$ carry favorable prognosis compared to Gleason score $3+4=7$ tumors with higher percentage $(>20 \%)$ of pattern 4 .

2. Percentage of GP4 is an important parameter that should be reported on all radical prostatectomies with Gleason score 7, given its independent prognostic value.

3. Additional studies are needed to determine the role of higher Gleason pattern percentages in tumors other than Gleason score 7.

956 Significance of Percentage of Gleason Pattern 4 at Needle Biopsy in Predicting Final Gleason Score and Correlation with Pathologic Outcomes at Radical Prostatectomy

Mohsin Jamal, Sean R Williamson, Mireya Diaz-Insua, Mani Menon, Hans Stricker, James Peabody, Craig G Rogers, Nilesh S Gupta. Henry Ford Hospital, Detroit, MI. Background: The 2005 ISUP modified Gleason grading scheme resulted in upgrade of some Gleason score (GS) 6 tumors to GS7. Most practices currently provide percentage of higher Gleason patterns in prostate needle biopsies (PNB), with the most common scenario being percentage of Gleason pattern 4 (\%G4). The aim of our study was to evaluate the clinical significance of reporting this finding.

Design: We analyzed PNBs between 2011 - 2013 with diagnosis of GS7 and 8 prostatic adenocarcinoma ( $\mathrm{PCa}$ ) and corresponding robotic radical prostatectomy (RRP) specimens.

Results: A total of 162 cases with both PNB and RRP specimens were selected. Mean age of these patients was 62 years. The $\% \mathrm{G} 4$ on biopsy correlated significantly with percentage of positive cores $(\mathrm{rho}=0.60, \mathrm{p}<0.001)$ and tumor volume $(\mathrm{rho}=0.52, \mathrm{p}<0.001)$. We stratified the patients into four quartiles based on $\% \mathrm{G} 4$ on PNB . Q1: $\% \mathrm{G} 4<=10$, Q2: $>10 \%-20 \%$, Q3: $>20 \%-50 \%$, Q4: $>50 \%$. Correlation of the $\% \mathrm{G} 4$ quartiles with the GS on RRP is listed in Table 1.

Table 1. Gleason score change from PNB to RRP by quartile of biopsy \%G4

\begin{tabular}{|c|c|c|c|c|c|c|}
\cline { 3 - 8 } \multicolumn{1}{c|}{} & \multicolumn{5}{c|}{ GS on RRP specimen } \\
\hline Quartile & $\begin{array}{c}\text { GS on } \\
\text { biopsy }\end{array}$ & $\mathbf{3 + 3 = 6}$ & $\mathbf{3 + 4 = 7}$ & $\mathbf{4 + 3 = 7}$ & $\mathbf{4 + 4 = 8}$ & $\mathbf{4 + 5}=\mathbf{9}$ \\
\hline Q1 & $3+4=7$ & 14.6 & 72.9 & 10.4 & 0 & 2.1 \\
\hline Q2 & $3+4=7$ & 4.8 & 69.1 & 23.8 & 0 & 2.4 \\
\hline Q3 & $3+4=7$ & 3.1 & 59.4 & 34.4 & 0 & 3.1 \\
\hline Q4 & $4+3=7$ & 0 & 23.8 & 66.7 & 4.8 & 4.8 \\
\hline Q4 & $4+4=8$ & 0 & 7.1 & 35.7 & 42.9 & 14.3 \\
\hline
\end{tabular}

Numbers represent percent of individuals within the quartile of the distribution of percent GP4 on biopsy with the GS on RRP.

For quartiles Q1, Q2 and Q3, there was a significant increase of upgrading in the RRP specimen to $4+3=7$ with increase of $\% \mathrm{G} 4$ on biopsy ( $\mathrm{p}-\mathrm{v}=0.005$ ). Likewise, within Q4, there was a significant upgrade of GS in the RRP specimen (from $4+3=7$ to $4+4=8$ and $4+5=9$ ).

Q1 was associated with greater percentage of organ confined disease, and negative lymph nodes (LN). In contrast, Q3 and Q4 were associated with high percentage of established extraprostatic extension, seminal vesicle invasion, angio invasion and positive LN (Table 2).

Table 2. Association of categorical pathology outcomes by quartiles of percentage of biopsy Gleason 4

\begin{tabular}{|c|c|c|c|c|c|}
\hline Outcome & Q1 $(n=48)$ & $Q 2(n=42)$ & Q3 $(n=33)$ & $\mathrm{Q}_{4}(\mathrm{n}=39)$ & p-value \\
\hline Organ confined & 64.6 & 42.9 & 36.4 & 38.5 & 0.010 \\
\hline Extraprostatic & 35.4 & 57.1 & 62.5 & 61.5 & 0.012 \\
\hline extension & 41.2 & 58.3 & 25.0 & 29.2 & 0.092 \\
\hline $\begin{array}{l}\text { Focal } \\
\text { Established }\end{array}$ & 58.8 & 41.7 & 75.0 & 70.8 & \\
\hline $\begin{array}{l}\text { Seminal vesicle } \\
\text { invasion }\end{array}$ & 2.1 & 2.4 & 15.2 & 15.4 & 0.006 \\
\hline Margin + & 22.9 & 9.5 & 18.2 & 28.2 & 0.434 \\
\hline Positive LN & 0 & 2.4 & 3.0 & 10.3 & 0.016 \\
\hline Angio invasion & 2.1 & 2.4 & 9.1 & 23.1 & $<0.001$ \\
\hline $\begin{array}{l}\text { Perineural } \\
\text { invasion }\end{array}$ & 6.3 & 2.4 & 6.1 & 0 & 0.227 \\
\hline Recurrence $^{1}$ & 4.3 & 0 & 6.5 & 15.8 & 0.020 \\
\hline
\end{tabular}

Numbers represent percent of individuals within the quartile of the distribution of percent biopsy Gleason 4 with the particular outcome. Q1: percent of biopsy Gleason $4<=10, Q 2:>10 \%-20 \%, Q 3$ : $>20 \%$ - 50\%, Q4: >50\%. p-value based on Mantel-Haenszel statistic. ${ }^{1}$ Four cases did not have recurrence information.

Conclusions: $\% \mathrm{G} 4$ is a clinically significant parameter that provides valuable information in management of patients with PCa. GS7 PCa with minor \%G4 shows favorable pathologic outcome, whereas there is an increase in upgrading and adverse pathologic outcomes with increase in $\% \mathrm{G} 4$ reported on PNB. 
957 Interobserver Agreement of Clear Cell Renal Cell Carcinoma (CCRCC) Grading Systems among Genitourinary Pathologists

Matt Jeffreys, Jesse K McKenney, Neriman Gokden, Shree G Sharma, Stephen M Bonsib, Christopher G Przybycin, Cristina Magi-Galluzzi, Jordan P Reynolds, Christina Stallworth, Jonathan Myles, Katherine Watts, Emily Holthoff, Andres A Roma, Roni M Cox. UAMS, Little Rock, AR; Nephropath, Little Rock, AR; Cleveland Clinic, Cleveland, $\mathrm{OH}$.

Background: Recent efforts to improve the prognostic significance and reproducibility of grading systems in renal cell carcinoma have resulted in alternatives to the Fuhrman grading system. At the International Society of Urologic Pathology (ISUP) consensus in Vancouver, a grading system based upon nucleolar prominence was instituted for clear cell and papillary renal cell carcinomas, which has since largely replaced the Fuhrman grading system. Recently, Delahunt et al proposed a modified grading system based on both ISUP grade and the presence or absence of tumor necrosis. In the current study, we examined the interobserver agreement between genitourinary pathologists for both nucleolar grading of the ISUP system and the presence or absence of necrosis for the newly proposed modified grading system in CCRCC.

Design: Fifteen H\&E stained slides of CCRCC were independently reviewed by 12 genitourinary pathologists, with each pathologist assigning an ISUP grade and evaluating for the presence or absence of tumor necrosis. Slides were selected to represent the spectrum of ISUP grades and included cases with rhabdoid and sarcomatoid differentiation.

Results: Kappa analysis on interobserver agreement of ISUP grading was 0.5156 (moderate agreement). Six cases had less than 9 reviewers agreeing on ISUP nucleolar grade. In two of these cases, the disagreement was based on the threshold for the degree of nuclear pleomorphism sufficient for ISUP grade 4 assignment. Two cases each had disagreement between classification as ISUP grade 1-2 and as ISUP grade 2-3. The kappa analysis for the presence or absence of necrosis was 0.7972 (strong agreement). Kappa analysis for the modified (ISUP grade + necrosis) grading system was 0.5724 (moderate agreement).

Conclusions: Moderate interobserver agreement was obtained with both ISUP grading of CCRCC and the proposed modified grading system that incorporates both ISUP grade and necrosis. Strong interobserver agreement was observed when evaluating for the presence or absence of tumor necrosis. The degree of nuclear pleomorphism required to assign an ISUP grade 4 was noted to be a problematic area. A mild increase in interobserver agreement was noted with the modified (nucleolar + necrosis) grading system as compared to the ISUP grading system.

958 Whole-Mounting of Prostates from Radical Cystoprostatectomies Enhances the Staging of Both Bladder and Prostate Cancers within the Specimens

Liwei Jia, Robin M Elliott, Gregory MacLennan. Case Western Reserve University, Cleveland, $\mathrm{OH}$.

Background: Histopathologic evaluation of the prostate in radical cystoprostatectomy specimens varies between institutions. Little data is available regarding the potential staging value of whole-mounting the prostate in cystoprostatectomy specimens. In recent years, we elected to begin whole-mounting the prostates in these cases. After a considerable experience in whole-mounting, we aimed to assess whether this technique provides more accurate staging data for both bladder cancer and prostatic adenocarcinoma identified in cystoprostatectomy specimens, as compared to routine processing with only representative sampling of the prostate.

Design: A retrospective search was performed to identify pathology reports from patients who had radical cystoprostatectomy for bladder cancer in our institution between 01/01/1995 and 08/31/2015. Prostates from cystoprostatectomies were routinely processed as standard representative sections from 1995 to 2011 and as whole mount sections from 2012 to 2015. Pathology reports of each case were reviewed. The histopathologic type and stage of bladder cancer were recorded for routinely processed and whole-mounted specimens. The cases with incidental prostatic adenocarcinoma were evaluated for Gleason score and stage, employing the same practices that are routinely applied to prostate cancer cases in our institution.

Results: A total of 159 cystoprostatectomy specimens were identified, including 105 routinely processed and 54 whole mounted specimens. In the routinely processed group, 86 cases showed invasive bladder cancer, 16 of which showed prostatic stromal invasion by bladder carcinoma, while 33 cases of invasive bladder were diagnosed in the wholemounted group, with prostatic stromal invasion by bladder carcinoma in 8 cases. Thus, the incidence of advanced bladder cancer (stage pT4) is $18 \%$ in the routinely processed group and $24 \%$ in the whole-mounted group. Incidental prostatic adenocarcinoma was identified in 24 cases (23\%) in the routinely processed group and 25 cases $(46 \%)$ in the whole-mounted group. Among these prostatic adenocarcinomas, 7 cases from the routinely processed group $(7 \%)$ and 8 cases from the whole-mounted group $(15 \%)$ were clinically significant, with Gleason score of 7 or higher.

Conclusions: Based on our experience, whole-mounting of the prostate in radical cystoprostatectomy specimens increases the incidence of identification of prostatic stromal involvement by bladder cancer (stage pT4), and the detection rate for clinically significant prostatic adenocarcinoma in these specimens.

959 Comparative Analysis of Neuroendocrine Phenotype in Metastases from Advanced Prostate Cancer and Their Corresponding Primary Tumor Rafael E Jimenez, Jad Abbas, John C Cheville, Manish Kohli. Mayo Clinic, Rochester, MN.

Background: Neuroendocrine differentiation (NED) in metastatic prostate cancer (MPC) is considered an aggressive phenotype with poor outcomes and limited treatment options. However, it is a common phenomenon in primary prostate cancer (PPC).
There is little information regarding the correlation of NED between the primary and metastatic tumor, as metastatic site biopsies (MSB) are not routinely performed during clinical care. We evaluated a matched archival set of MSB with their corresponding PPC to determine patterns of NED in in both settings.

Design: We identified MPC patients (pts) who underwent MSB during routine clinical care and who had a PPC sampled at our institution. Whole sections were analyzed for MPC specimens, while a tissue microarray (TMA) was constructed for the PPC. Immunohistochemical analysis was performed for expression of chromogranin-A and synaptophysin, and graded as $0,1+, 2+$, or $3+$, according to staining in none, $<10 \%, 11-$ $50 \%$, or $>50 \%$ of tumor cells, respectively. Highest score was recorded when discrepant results between the two stains were obtained.

Results: Out of 187 pts with a MSB at our institution (1994-2013), 36 had adequate primary tissue in our archives to construct a TMA. Five cases were excluded, as the MSB did not have enough tissue for subsequent analysis. PPC was evaluated in material from radical prostatectomies (25), cystoprostatectomy (2) transurethral resection (2), and needle core biopsy (1). MSB included bone (12), liver (7), lung (6), bladder (2), soft tissue (2), brain (2). In the PPC, staining was observed in $21(68 \%)$ cases (15 1+, $12+, 53+)$. On the MPC, staining was observed in $14(45 \%)$ cases $(51+, 52+, 43+)$. Of 25 PPC with a $0 / 1+$ score, $20(80 \%)$ had a $0 / 1+$ MPC, while 5 had $>2+$ MPC. Of the 6 PPC with $\mathrm{a} \geq 2+$ score, 3 had $0 / 1+$ and $3 \geq 2+$ MPC (p=NS). Of 9 MPC with $\geq 2+$, $6(66 \%)$ had $0 / 1+$ expression on the PPC.

Conclusions: While statistical significance was not reached due to small number of $\geq 2+$ PPC cases, the data suggest that absent/focal NED is more commonly associated with absent/focal NED in the metastatic setting. However, diffuse NED may not develop until the metastatic progression of prostate cancer.

\section{ERG and Prostein Expression in Prostate Cancer Identify Different} Pathways of Progression

Nuria Juanpere, Josep Lloreta, Alba Font, Marta Lorenzo, Raquel Albero, Ivonne Vazquez, Lluis Fumado, Lluis Cecchini, Sergio Serrano, Silvia Hernandez. Hospital del Mar-PSMar, Barcelona, Spain; Pompeu Fabra University, Barcelona, Spain; Autonomous University of Barcelona, Barcelona, Spain.

Background: $E R G$ is the most frequent partner of TMPRSS2 rearrangement, and $S L C 45 A 3$ (prostein), related to prostatic differentiation, is the second most common. Its rearrangement results in loss of prostein expression. The aim of the present study has been to analyze the relationship between the immunohistochemical (IHC) expression of ERG and prostein and clinical-pathological variables of PCa.

Design: 221 PCa distributed in 9 TMA were included: 39 cases with GS=6, 104 with $\mathrm{GS}=7$, and 78 with $\mathrm{GS} \geq 8$. IHC expression was assessed with an anti-ERG monoclonal $\mathrm{Ab}$ (EPR3864, Epitomics, Burlingame, CA, USA) and an anti-prostein monoclonal Ab (10E3, DAKO, Glostrup Denmark). Biological samples were obtained from PSMAR Biobank. Clinical-pathological endpoints were GS and PSA-progression free survival. Results: ERG expression was detected in $103(46.6 \%)$ and prostein loss in $71(32.1 \%)$ PCa. ERG expression did not vary across the different GS categories $(\mathrm{p}=0.52)$. Conversely, prostein loss was more frequent in cases with higher GS [5/39 with GS $=6$ $(12 \%), 32 / 104$ with $\mathrm{GS}=7(30.7 \%)$ and $37 / 78(43.6 \%)$ with $\mathrm{GS} \geq 8 \mathrm{PCa}(\mathrm{p}=0.003)$. The association ERG+/prostein loss was significantly related to grade [21/143 (14.7\%) PCa with $\mathrm{GS} \leq 7$ vs $20 / 78(25.5 \%)$ with $\mathrm{GS} \geq 8](\mathrm{p}=0.045)$. The coexistence of $E R G$ positivity and preserved prostein expression was significantly less common in high grade $\mathrm{PCa}$ $[12 / 39(30.8 \%)$ cases with $\mathrm{GS}=6,33 / 104(31.7 \%)$ with $\mathrm{GS}=7$ and $17 / 78(21.8 \%)$ with $\mathrm{GS} \geq 8] \quad(\mathrm{p}=0.012)$. In $\mathrm{GS} \geq 8 \mathrm{PCa}, \mathrm{ERG}$ expression was more often associated with Gleason pattern $3: 12 / 36(66.6 \%) \mathrm{GS} \geq 8 \mathrm{PCa}$ with pattern 3 and $13 / 42(30.9 \%) \mathrm{GS} \geq 8 \mathrm{PCa}$ without it were $\mathrm{ERG}+(\mathrm{p}=0.001)$. $\mathrm{ERG}+$ /prostein loss was detected in $13 / 36(36 \%) \mathrm{GS} \geq 8$ $\mathrm{PCa}$ with pattern 3 compared to $7 / 41$ ( $17 \%$ ) $\mathrm{GS} \geq 8 \mathrm{PCa}$ without it $(\mathrm{p}=0.057)$. $\mathrm{ERG}+$ cases were associated with shorter PSA-progression free survival ( $\log$ Rank test $\mathrm{p}=0.022$ ). Conclusions: ERG expression is associated with shorter PSA-progression free survival. ERG + cases with normal prostein tend to be of lower GS. Prostein loss relates to higher GS PCa, usually combined with ERG + . The association of ERG + with the presence of pattern 3 in higher GS PCa suggests that progression from pattern 3 to 4 is more common in rearranged $\mathrm{PCa}$ and that non-rearranged cases would more often be de novo pattern 4 . Supported by FIS/Instituto Carlos III/FEDER PI12/01426, FEDER PT13/0010/0005, Spanish Ministry of Health, and the "Xarxa de Bancs de tumors, Pla Director d'Oncologia de Catalunya (XBTC)

961 Immunohistochemistry of Prostaglandin Receptors in Bladder Cancer as Predictors of Tumor Progression as Well as Chemosensitivity Eiji Kashiwagi, Hiroki Ide, Satoshi Inoue, Takashi Kawahara, George JNetto, Alexander Baras, Hiroshi Miyamoto. Johns Hopkins University, Baltimore, MD.

Background: Prostaglandin E2 (PGE2) mediates its effects by activating the G proteincoupled E-prostanoid (EP) receptors (e.g. EP2, EP3, EP4). Recent evidence indicates that these receptors mediate the tumor promoting activity of PGE2 in various malignancies. The current study aims to determine the expression status of EP receptors in bladder cancer and its prognostic significance.

Design: We immunohistochemically stained for EP2/EP3/EP4 in 129 bladder tumor and matched non-neoplastic bladder tissue specimens as well as for EP2/EP4 in separate tissue microarrays consisting of 37 muscle-invasive bladder cancer specimens from patients who received at least 3 cycles of cisplatin + gemcitabine neoadjuvant chemotherapy.

Results: EP2/EP3/EP4 was positive in 70\%/87\%/74\% of tumors, which was significantly higher than in benign urothelial tissues $[23 \%(P<0.001) / 70 \%(P=0.003) / 43 \%$ $(P<0.001)]$. Twenty-five $(49 \%)$ of 51 lower grade tumors vs. 65 (83\%) of 78 high-grade carcinomas $(P<0.001)$ and $47(60 \%)$ of 78 non-muscle-invasive tumors $v s .43(84 \%)$ of 51 muscle-invasive carcinomas $(P=0.006)$ were immunoreactive for EP2. Similarly, 62 $(84 \%)$ of 78 non-muscle-invasive tumors vs. 50 (98\%) of 51 muscle-invasive carcinomas 
were immunoreactive for EP3 ( $P=0.002)$. However, there were no statistically significant associations between EP4 positivity and tumor grade or $\mathrm{pT}$ stage as well as between expression pattern of each receptor and $\mathrm{pN}$ status. Kaplan-Meier and log-rank tests revealed that positivity of EP2 $(P=0.035)$ or EP4 $(P=0.027)$ in patients with muscleinvasive tumor strongly correlated with disease-specific mortality. Multivariate Cox model showed a trend toward significance in EP2 positivity ( $\mathrm{HR}=6.452,95 \% \mathrm{CI}=0.864-$ $48.179, P=0.069)$ and significance in $\mathrm{EP} 4$ positivity $(\mathrm{HR}=5.604,95 \% \mathrm{CI}=1.283-24.475$, $P=0.022)$ as independent predictors for disease-specific survival. In a separate set of tissue specimens, EP2/EP4 was positive in $31(84 \%) / 30(81 \%)$ of 37 cases with chemotherapy, including $11(69 \%) / 11(69 \%)$ of 16 responders vs. $20(95 \%) / 19(90 \%)$ of 21 non-responders, respectively. In particular, moderate to strong positivity of EP2 $(P=0.008)$ or EP4 $(P=0.099)$ was associated with chemoresistance.

Conclusions: Compared with non-neoplastic urothelium, significant increases in the expression of EP receptors in bladder tumor were observed. Overexpression of EP2 and EP3 was strongly associated with high-grade and/or muscle-invasive tumors. Our results also suggest that EP2 or EP4 expression serves as a predictor of prognosis after radical cystectomy as well as chemosensitivity.

\section{Genomic Analysis of Pure Adult Yolk Sac Tumor of the Testis}

Jennifer Katzenberg, John A Thorson, Thomas M Ulbright, Donna Hansel. University of California at San Diego, San Diego, CA; Indiana University, Indianapolis, IN. Background: Pure adult yolk sac tumors (YST) of the testis are rare. In contrast to type I germ cell tumors (GCTs) that occur in children and include pure YST, the adult pure YST counterpart is considered a type II GCT with derivation of the YST element from a precedent embryonal carcinoma (EC). Prior molecular analysis has focused on assessment of transdifferentiation of GCT elements. In this study, we analyzed 3 pure adult YST using a 397-gene analysis panel to identify putative pathways in development of this rare tumor type.

Design: DNA was extracted from archived formalin-fixed, paraffin embedded blocks followed by fragmentation and indexed adapter ligation.DNA sequences of interest were enriched with a custom Agilent hybrid capture probe set, then sequenced on an Illumina HiSeq 2500 using 101 bp reads. A custom bioinformatics pipeline was used to identify variants and query the 1000 Genomes database to aid in the identification of germline variants and the COSMIC and cBioportal databases for known cancer association(s). SIFT and PolyPhen-2 predictions were used to evaluate variants of uncertain significance (VUS) for possible deleterious effects on protein structure or function. Slides and immunohistochemistry were re-reviewed to confirm the diagnosis. Results: Age, AFP (ng/mL) and tumor size for patients included: Patient 1, 36 yo, AFP 8360, $9.0 \mathrm{~cm}$; Patient 2, 20 yo, AFP 46, $3.9 \mathrm{~cm}$; Patient 3, 47 yo, AFP 10925, 10.5 $\mathrm{cm}$. All 3 tumors showed probable somatic mutation of the DNA mismatch repair gene MSH6 and germline mutation of the ERCC5 DNA repair enzyme. All 3 tumors also showed gene mutations that influence histone modification, including histone demethylase (KDM5A; somatic 1 patient) and histone acetylation (IL7R, germline 3 patients; $K A T 6 B$, germline 1 patient and somatic 1 patient; $C R E B B P$, somatic 1 patient). Pathway activation of both PPARgamma signaling (PPARgamma and THRAP3 somatic mutation) and adherens junction/Wnt/APC pathway (CTNNB1, FAT1, RNF43 somatic mutations) were also observed. NOTCH1, NOTCH3 and KRAS were mutated in individual patients.

Conclusions: Our data suggests that defects in DNA repair and altered histone modification are common in pure adult YST and may contribute to the pathogenesis of unusual tumor.

963 ELK1 Immunohistochemistry in Bladder Cancer as a Predictor of Tumor Progression as Well as Chemosensitivity

Takashi Kawahara, Hiroki Ide, Hasanain Shareef, Satoshi Inoue, Eiji Kashiwagi, George J Netto, Alexander Baras, Hiroshi Miyamoto. Johns Hopkins University, Baltimore, MD. Background: A transcriptional factor ELK1 is phosphorylated and activated via the MAPK/ERK pathways. Using cell line and animal models, we recently found that ELK1 signals not only promoted bladder cancer growth but also reduced the cytotoxic activity of chemotherapeutic drugs, including cisplatin. The current study aims to determine the expression status of ELK1 and phospho-ELK1 (p-ELK1) in bladder cancer and its prognostic significance.

Design: We immunohistochemically stained for ELK1 and p-ELK1 in 129 bladder tumor and paired non-neoplastic bladder tissue specimens as well as for p-ELK1 in separate tissue microarrays consisting of 37 muscle-invasive bladder cancer specimens from patients who received 3 (without dose reduction) or $\geq 4$ (with or without dose reduction) cycles of cisplatin + gemcitabine neoadjuvant chemotherapy.

Results: ELK1/p-ELK1 was positive in $100 \%(25 \% 2+, 75 \% 3+) / 66 \%(36 \% 1+, 15 \%$ $2+, 16 \% 3+$ ) of tumors, which was significantly higher than in non-neoplastic urothelial tissues $[100 \%(8 \% 1+, 37 \% 2+, 55 \% 3+) / 35 \%(21 \% 1+, 12 \% 2+, 2 \% 3+)]$. There were no statistically significant differences in ELK1 or p-ELK1 levels between male versus female tumors, low-grade versus high-grade tumors, and $\mathrm{pN} 0$ versus $\mathrm{pN}+$ tumors. Kaplan-Meier and log-rank tests revealed that patients with p-ELK1-positive nonmuscle-invasive and $\mathrm{p}$-ELK1-positive muscle-invasive tumors had significantly higher risks of tumor recurrence $(P=0.043)$ and disease progression $(P=0.045) /$ cancer-specific mortality $(P=0.008)$, respectively. In contrast, no significant associations between ELK1 levels in these tumors and patient outcomes were found. Multivariate analysis further identified p-ELK1 positivity in muscle-invasive tumors as an independent predictor of cancer-specific mortality hazard ratio $(\mathrm{HR})=2.693,95 \%$ confidence interval $(\mathrm{CI})=1.164$ $6.229, P=0.021]$. There was also a trend toward significance between $\mathrm{p}$-ELK1 positivity in non-muscle-invasive tumors and tumor recurrence $(\mathrm{HR}=2.829,95 \% \mathrm{CI}=0.976-8.204$, $P=0.056)$. In a separate set of tissue specimens, p-ELK1 was positive in $21(57 \%)$ of
37 cases with chemotherapy, including $6(38 \%)$ of 16 responders versus $15(71 \%)$ of 21 non-responders. Thus, $\mathrm{p}$-ELK1 positivity significantly $(P=0.039)$ correlated with chemoresistance.

Conclusions: ELK1 is likely to be activated in most of bladder cancers, supporting our preclinical findings. The current results also suggest that p-ELK1 positivity precisely predicts recurrence of non-muscle-invasive tumor, progression of muscle-invasive tumor, and, more interestingly, chemoresistance in patients with muscle-invasive tumor.

964 Vasitis Nodosa and Related Lesions: A Modern Immunohistochemical Staining Profile with Special Emphasis on Diagnostic Dilemmas Brie Kezlarian, Liang Cheng, Nilesh S Gupta, Sean R Williamson. Henry Ford Health System, Detroit, MI; Indiana University, Indianapolis, IN.

Background: Vasitis nodosa is a benign proliferation of vas deferens epithelium, thought to be a response to trauma, obstruction, or previous surgical intervention, usually vasectomy. Although diagnosis is usually straightforward, worrisome histologic features are well-known, such as prominent nucleoli, pseudoinvasive growth pattern, and perineural invasion. We sought to characterize the immunohistochemical staining pattern with a modern antibody panel, with particular emphasis on cases occurring in worrisome clinical scenarios.

Design: We queried our database for cases of vasitis or epididymitis nodosa or sperm granuloma, yielding a cohort of 28 specimens. Ultimately, 21 were confirmed to have vasitis nodosa and were included in the final cohort. These, in addition to two posttreatment prostate and bladder carcinomas mimicking vasitis nodosa were stained with antibodies to PAX-8, CD10, p63, racemase (AMACR), prostein and prostate-specific antigen (PSA)

Results: Two diagnostically problematic cases of vasitis nodosa included 1) bladder muscle and florid soft tissue involvement in a cystectomy specimen after prostatectomy and 2) involvement of the ampulla and ejaculatory duct in a radical prostatectomy specimen. PAX-8 yielded consistent positive (100\%) nuclear staining in the lesional glands, often stronger and more uniform than native vas deferens. CD10 similarly labeled the proliferative glands, in addition to luminal and extra-cellular secretions, and the basement membranes and apical surfaces of native and proliferative glands. Labeling for p63 was basally located, but often markedly attenuated or lacking in the proliferative glands compared to native epithelium. Racemase positivity was variable but often present (18/21). PSA and prostein were consistently (100\%) negative.

Conclusions: In addition to characterizing the immunohistochemical signature of vasitis nodosa, this study unearthed novel diagnostic pitfalls. Rare problematic lesions include "invasion" of the ejaculatory duct at the prostate and involvement of bladder muscle after prostatectomy. The proliferative vasitis nodosa glands often have a prostate cancer-like staining pattern with variable racemase positivity and negative or patchy p63, in contrast to the native vas deferens. However, reliable positivity for PAX-8 and negative staining for PSA and prostein aid in distinguishing from prostate cancer.

\section{Copper Transporter CTR1 Expression Predicts Platinum Response} in Muscle-Invasive Bladder Cancer

Deepak Kilari, Kenneth A Iczkowski, Chintan Pandya, Adam Robin, Edward M Messing, Elizabeth Guancial, Eric S Kim. Medical College of Wisconsin, Milwaukee, WI; University of Rochester, Rochester, NY.

Background: Platinum (Pt)-based neoadjuvant chemotherapy (NAC) is the standard of care for muscle-invasive bladder cancer (MIBC). However, at least half of patients do not respond to NAC. Non-responders are subject to the adverse effects of Pt, with delay in definitive treatment. Copper Transporter Receptor 1 (CTR1) imports Pt into cells while CTR2, ATP7A, and ATP7B export Pt; their levels of expression may influence Pt sensitivity in MIBC.

Design: We identified matched paraffin-embedded tissue from pre-NAC transurethral bladder tumor resection (TURBT) and post-NAC radical cystectomy (RC) specimens in 47 patients with MIBC who received Pt-based NAC. None of the patients had receieved chemotherapy for any malignancy prior to the diagnosis of urinary bladder cancer. Tumor and adjacent normal tissues ( $\geq 3$ cores each on tissue microarrays) were stained with CTR1 antibody. CTR1 expression was determined through immunohistochemistry by two pathologists blinded to the outcome $(0=$ none; $1+=$ rare, $<10 \%$ cells; $2+=$ moderate, $11-50 \%$ cells; and $3+=$ intense in $>50 \%$ cells). Pathologic response was defined as either down-staging to non-MIBC $(\leq \mathrm{pT} 1 \mathrm{~N} 0 \mathrm{M} 0)$ or complete pathologic response (pT0). Pathologic outcome was compared between CTR1 expression groups. Results: $21 / 47$ (44\%) of patients showed pathologic response to chemotherapy. $8 / 47$ $(17 \%)$ had pT0 cancer at cystectomy. Wilcoxon sign-rank test was performed on matched samples dichotomized into high expression $(3+)$ versus lower expression $(\leq 2+) .43 \%$ of TURBT and $41 \%$ of RC specimens expressed a CTR 1 score of $3+$. In both pre-NAC TURBT and post-NAC RC specimens, CTR1 expression score of $3+$ correlated with pathologic response ( $\mathrm{p}=0.0076$ and $\mathrm{p}=0.023$, respectively). 18 of 25 (72\%) patients with CTR 1 expression of $\leq 2$ had $\geq p T 2$ cancer despite NAC. No difference in CTR1 expression was evident between matched pre-NAC and post-NAC specimens. Expression of CTR2, ATP7A, and ATP7B, and of CTR1 in benign urothelium did not predict response.

Conclusions: This is the first study demonstrating a correlation between tumor CTR1 expression and pathologic outcome in Pt-treated MIBC. These findings suggest that CTR1 expression may be a biomarker for Pt sensitivity. 
966 Renal Tumors with Papillary Morphology

Max Kong, Jianhong Li, Fang-Ming Deng, Peng Lee, Jonathan Melamed, Ming Zhou. New York University, New York, NY

Background: Histological classification of renal tumors is important for prognostic and therapeutic considerations. Papillary morphology, focal or diffuse, is common and can be seen in many different types of renal tumors. It is very important to remember that not all renal tumors with papillary morphology or a papillary component are papillary renal cell carcinoma (PRCC). We studied the incidence of papillary morphology in renal tumors and morphological features that are associated with PRCC.

Design: Partial and radical nephrectomies performed in 2014 were reviewed for diagnosis, histological classification, pathological characteristics of the tumor (tumor capsule, histiocyte aggregates, psammoma calcification), presence and amount (\%) of papillary morphology, and the morphology of areas adjacent to papillary architecture (solid, cystic, necrosis).

Results: Of 149 renal tumors reviewed, there were 77 (52\%) clear cell RCC (CCRCC), $32(21 \%)$ PRCC, 9 (6\%) chromophobe RCC (ChRCC), $4(3 \%)$ clear cell tubulopapillary RCC (CCTPRCC), 14 (9\%) oncocytoma (Onco), 10 (7\%) unclassified RCC and 5 (3\%) other rare tumors including tubulocystic RCC and metanephric adenoma. Papillary architecture was present in $103(69 \%)$ of cases, including $47 / 77$ (61\%) CCRCC, $32 / 32$ (100\%) PRCC, $7 / 9$ (78\%) ChRCC, 4/4/(100\%) CCTPRCC and 5/14 (36\%) Onco. The mean percentage of papillary component was $28.3 \%$ (range $1-100 \%$ ) in all tumors, $11.8 \%$ $(1-90 \%)$ in non-PRCC, $64.8 \%(5-100)$ in PRCC. In non-PRCC tumors, the papillary components accounted for $<10,10-49$ and $\geq 50 \%$ of the tumor volume in $45(63 \%)$, $21(30 \%)$ and $5(7 \%)$ cases. In 49/71 (69\%) of these cases, papillary morphology was adjacent to cystic and necrotic areas. Of the 3 histological features (tumor capsule, histiocyte aggregates, psammoma calcification), 65/71 of non-PRCC and 3/32 of PRCC had none or 1 feature, while 6/71 non-PRCC and 29/32 PRCC had $\geq 2$ features $(p<0.001)$. Conclusions: Papillary morphology is common in non-PRCC renal tumors, some of which have extensive papillary architecture. In majority of the cases, the papillary morphology is seen next to cystic and necrotic areas. Presence of $>2$ of the histological features (tumor capsule, histiocyte aggregates, psammoma calcification) is strongly suggestive of PRCC diagnosis.

967 Assessment of Differences in Prostate Cancer Characteristics between Asian and Non-Asian Men

Pavel Kopach, Elizabeth Genega. Tufts Medical Center, Boston, MA.

Background: Evaluation of prostate cancer (PCa) biological behavior has shown racial differences in disease onset and progression. Yet, there is a paucity of data on clinicopathologic and prognostic characteristics of PCa among Asian men (AM). While the incidence of $\mathrm{PCa}$ is reported to be lower in $\mathrm{AM}$ than other ethnic groups, there is still controversy regarding the prognosis and outcome in AM population compared to non-Asian (NAM). It remains unclear whether there is an intrinsic biological or genetic difference in PCa of AM.

Design: The surgical pathology files were searched from 2005 to 2008 for patients with prostate biopsies performed at our institution. We evaluated the incidence, clinicopathologic characteristics and management of PCa in AM compared to NAM. Results: We analyzed 500 men, of which 168 (33.6\%) were AM and 332 (66.4\%) were NAM. Patients underwent prostate needle biopsy due to increased PSA level $(92.9 \%$ AM; $84.9 \%$ NAM) or abnormal rectal exam (7.1\% AM; $15.1 \%$ NAM). AM (47.5\%) presented at significantly older age (age $\geq 71)$ compared to NAM $(22.5 \% ; \mathrm{P}<0.01)$. The majority of NAM presented at age less than $70(60.5 \%)$ and a greater number of NAM $(32.2 \%)$ were at age less than 60 compared to AM (14.3\%). AM showed significantly higher preoperative serum PSA (mean $7.59 \mathrm{ng} / \mathrm{mL}, 95 \%$ CI 6.8-8.4), than NAM (mean $6.4 \mathrm{ng} / \mathrm{mL}, 95 \% \mathrm{CI} 5.8-6.9 ; \mathrm{P}<0.05$ ), but AM had a significantly lower incidence of $\mathrm{PCa}$ $(36.9 \%, \mathrm{n}=62)$ compared to NAM $(59.9 \%, \mathrm{n}=199, \mathrm{P}<0.01)$. The proportion of patients choosing surgical treatment was higher among NAM than in AM $(\mathrm{P}<0.05)$. For those patients who underwent radical prostatectomy ( $\mathrm{AM}=27.4 \%$; $\mathrm{NAM}=42.7 \%$ ), there was no statistical difference in Gleason score between both groups $(\mathrm{P}>0.05)$. High-grade disease $(\geq 8)$ on prostatectomy was present in $9.5 \%(8 / 85)$ of NAM and $5.9 \%(1 / 17)$ in AM. There was also no significant difference in pathologic $\mathrm{T}$ stage between AM and NAM $(\mathrm{P}>0.05)$. Bone metastases were identified in $3.2 \%$ of AM and $3.0 \%$ of NAM $(\mathrm{P}>0.05)$. Among the patients who underwent non-surgical treatment, AM had significantly higher post-treatment serum PSA (mean $3.42 \mathrm{ng} / \mathrm{mL}, 95 \%$ CI 2.7-4.1) compared to NAM (mean $2.43 \mathrm{ng} / \mathrm{mL}, 95 \%$ CI 1.9-2.8; P $<0.05$ ). There was no PCa related mortality in either group.

Conclusions: AM and NAM men demonstrated significant differences in disease incidence, age at diagnosis, pre- and post-treatment PSA levels. Despite higher preoperative PSA level the majority of pathologic diagnoses in AM were benign compared to NAM, indicating that preoperative PSA may not be useful for predicting $\mathrm{PCa}$ among certain ethnic groups.

968 High Fidelity of Driver Chromosomal Alterations among Primary and Metastatic Renal Cell Carcinomas: Implications for Disease Evolution and Treatment

Erik Kouba, Novae Simper, John N Eble, David J Grignon, Mingsheng Wang, Shaobo Zhang, Lisha Wang, Guido Martignoni, Sean R Williamson, Matteo Brunelli, Claudio Luchini, Anna Calio', Liang Cheng. Indiana University School of Medicine, Indianapolis, IN; Fudan University Shanghai Cancer Center, Shanghai, China; Universitá di Verona, Verona, Italy; Henry Ford Health System, Detroit, MI.

Background: Recent studies have demonstrated considerable genomic heterogeneity in both primary and metastatic renal cell carcinomas (RCCs). This mutational diversity has serious implications for the development and implementation of targeted molecular therapies. We evaluated 32 cases of primary RCC with their associated metastatic tumors to determine if the hallmark chromosomal anomalies of these tumors are preserved over the course of disease progression.

Design: Thirty two matched pairs of primary and metastatic renal cell carcinomas (21 clear cell RCC, 11 papillary RCC) were analyzed. All tumors were evaluated for chromosome $3 p$ deletion and trisomy 7 and 17 using fluorescence in situ hybridization (FISH).

Results: Of the 21 clear cell RCCs, 17 primary tumors $(81 \%)$ showed a deletion of chromosome 3p. All 17 of the corresponding metastatic tumors displayed the same abnormality. Two additional tumors developed $3 p$ deletion in the metastasis. All clear cell RCCs were disomic for chromosomes 7 and 17. In contrast, 9 of the 11 papillary RCCs (82\%) showed trisomy for both chromosomes 7 and 17 . These molecular aberrations were conserved in the paired metastatic tumors.

Conclusions: Our results demonstrated a high degree of genomic fidelity among the primary and metastatic lesions in renal cell carcinomas. These findings may have important clinical and diagnostic implications.

969 Radical Prostatectomy (RP) Findings in Hispanic Men Qualifying for Active Surveillance (AS)

Oleksandr N Kryvenko, Kirill Lyapichev, Felix M Chinea, Nachiketh S Prakash, Alan Pollack, Sanoj Punnen, Merce Jorda. University of Miami, Miami, FL.

Background: The Epstein AS criteria were developed predominantly in Whites and have shown inferiority in Blacks. Hispanics were not tested.

Design: Analysis of RP in Hispanics with T1c Gleason score 6 PC ( $\leq 2$ cores) in template biopsy, $\mathrm{PSA}<10$. Hispanic ethnicity was identified by the North American Association of Central Cancer Registries criteria in the National Hispanic Identification Algorithm. T-test, Wilcoxon rank-sum, and Fisher's test were used.

Results: In 18 of 71 men PSA density was $>0.15$ - 3 of these had insignificant PC (2 had severe inflammation) and 15 had significant PC defined by volume (4), grade (1), grade\&volume (10). Remaining 53 men are summarized in table. No patient had lymph node metastasis. One had vanishing PC. Twenty (38\%) had significant PC defined by volume (8), grade (8), volume \& grade (4). Six men had tertiary pattern 4,3 had $3+4=7,1$ had $3+5=8,1$ had $4+5=9$. In cases with higher RP grade the dominant tumor nodule was anterior in $5(42 \%)$ and posterolateral and not the biggest tumor nodule in $3(25 \%)$ cases. Remaining 5 cases had posterolateral small volume dominant tumor nodules (mean, $0.27 \mathrm{ml}$; range, $0.09-0.45$ ).

\begin{tabular}{|c|c|c|c|c|c|}
\hline & \multirow{2}{*}{ All (53) } & \multicolumn{3}{|c|}{ Study; median (mean, range) } \\
\hline & & & Insignificant (33) & Significant (20) & $\mathrm{p}$ \\
\hline \multicolumn{2}{|l|}{ Age } & $61(59,43-70)$ & $59.0(59.0,43-70)$ & $61.5(59.1,43-69)$ & 0.96 \\
\hline \multicolumn{2}{|l|}{ PSA } & $\begin{array}{l}4.35(4.18,0.3- \\
9.25)\end{array}$ & $3.8(3.98,0.3-9.25)$ & $4.75(4.51,1.4-7.3)$ & 0.36 \\
\hline \multicolumn{2}{|l|}{ BMI } & $27(26.9,18.6-35.4)$ & $27(27.3,18.6-35.4)$ & $26.2(26.1,20.7-31)$ & 0.24 \\
\hline \multicolumn{2}{|c|}{$\begin{array}{l}\text { Prostate weight, } \\
\mathrm{gm}\end{array}$} & $44.9(51.1,20-158)$ & $41(52.6,20-158)$ & $45(48.6,31-97)$ & 0.77 \\
\hline \multicolumn{2}{|c|}{$\begin{array}{l}\text { Total tumor } \\
\text { volume, } \mathrm{ml}\end{array}$} & \begin{tabular}{|l|}
$0.276(0.493$ \\
$0.004-2.977)$
\end{tabular} & $\begin{array}{l}0.102(0.168,0.004- \\
0.612)\end{array}$ & $\begin{array}{l}0.793(1.012, \\
0.138-2.977)\end{array}$ & $<0.01$ \\
\hline \multicolumn{2}{|l|}{ PSAD } & \begin{tabular}{|l|}
$0.071(0.085$ \\
$0.015-0.148)$ \\
\end{tabular} & $\begin{array}{l}0.064(0.077,0.015- \\
0.147)\end{array}$ & $\begin{array}{l}0.11(0.097,0.021- \\
0.148)\end{array}$ & 0.049 \\
\hline \multicolumn{2}{|c|}{ PSA mass, $11 / 4 \mathrm{~g}$} & \begin{tabular}{|l|}
$0.493(0.483$ \\
$0.037-1.076)$
\end{tabular} & $\begin{array}{l}0.456(0.453,0.037- \\
1.076)\end{array}$ & $\begin{array}{l}0.519(0.532, \\
0.176-0.957)\end{array}$ & 0.24 \\
\hline \multicolumn{2}{|c|}{$\begin{array}{l}\text { PSA mass } \\
\text { density, } \hat{1}^{1} / 4 \mathrm{~g} / \mathrm{gm}\end{array}$} & \begin{tabular}{|l|}
$0.0093(0.0098$, \\
$0.0019-0.0195)$
\end{tabular} & \begin{tabular}{|l|}
$0.0079(0.0088$, \\
$0.0019-0.0161)$
\end{tabular} & $\begin{array}{l}0.012(0.0114 \\
0.0039-0.0195)\end{array}$ & 0.027 \\
\hline \multicolumn{2}{|c|}{$\begin{array}{l}\text { Anterior } \\
\text { dominant }\end{array}$} & 17 & 6 & 11 & 0.0075 \\
\hline \multirow{3}{*}{ Stage } & pT2 & 43 & 30 & 13 & \multirow{3}{*}{0.012} \\
\hline & pT2+ & 5 & 2 & 3 & \\
\hline & pT3 & 4 & 0 & 4 & \\
\hline
\end{tabular}

Conclusions: PSA density threshold of 0.15 works well in Hispanic men. Performance of the contemporary AS criteria is better than in Black and worse than White men. Large volume anterior disease could be reliably detected by multiparametric MRI before enrolling patients in AS and missed small volume posterolateral PC should not pose immediate harm and would likely be detected on the repeat template biopsies. PSA mass density accounting for BMI and prostate weight showed the best performance in distinguishing men with significant PC.

970 Multi-Tumor Sampling Tissue Microarray Approach from Radical Prostatectomies to Screen Biomarkers Relevant to Active Surveillance Ibrahim Kulac, Gunes Guner, Helen Fedor, Stephanie Glavaris, Tamara Lotan, H Ballentine Carter, Jonathan I Epstein, Angelo M De Marzo. Johns Hopkins University, Baltimore, MD.

Background: Active surveillance (AS) is safe for the majority of men with very low risk prostate cancer ( $\mathrm{Pca}$ ). Yet, some leave the AS program as a result of "progression" that is defined as an increased volume or a higher Gleason score (GS) on surveillance biopsy. While most of the men with progression have favorable pathology at radical prostatectomy (RP), very few ( $\mathrm{N}=32$ out of 1298 total men enrolled in AS) were found to have life threatening disease, defined as GS $>=4+3=7$; or GS $3+4=7$ with either SV invasion or tertiary Gleason Pattern (GP) 5. A TMA was constructed from these "escape" cases in order to find biomarkers that may be helpful to predict which patients with apparent low GS on biopsy harbor much more aggressive disease. Since 
PTEN loss on GS6 needle biopsies has been shown to be associated with upgrading in RP specimens (PMID: 24993522), we sought to determine the rate of PTEN loss in different GP components of "escapes".

Design: 26 escape "cases" and a matched group of "controls" also meeting the criteria for AS who underwent RP that had GS6 organ confined Pca were included. A TMA was constructed where each tumor nodule from escape "cases" was mapped in 3-D and sampled at least twice with a $0.6 \mathrm{~mm}$ core. If a tumor nodule was composed of different grades, areas with different GPs were sampled separately when possible. Both dominant and secondary nodules (if present) of controls were also sampled.

Results: In dominant nodules from the escape cases $(\mathrm{N}=26)$, PTEN loss occurred in 5 of the GP4 or mixed GP3/4 but in none of the pure GP3. 2 of the secondary nodules (all=GS7) had PTEN loss and none of the additional nodules showed PTEN loss in the Escape cases. In the dominant and secondary nodules of controls only 1 tumor showed PTEN loss ( $p=0.021$ comparing rate of PTEN loss in cases vs. controls)

Conclusions: PTEN loss was associated with a higher GS, as shown previously. Yet, loss was relatively uncommon in the GP3 component of the dominant and secondary nodules in escape cases. Thus, while the risk of unsampled higher grade disease is $\sim 3 \mathrm{x}$ higher if PTEN loss is found on a GS6 biopsy (PMID:2632359), PTEN loss in isolated GP3 was relatively infrequent in the minute areas sampled for this study. This will likely translate into a low negative predictive value for detecting aggressive disease in very low volume GP3 only biopsies. Additional markers with proposed high predictive ability for finding unsampled high grade disease are needed if samples with very low volume disease are used.

971 Gleason Grade 4 Prostate Cancer Growth Patterns: An Interobserver Study among Expert Genitourinary Pathologists

Charlotte Kweldam, Daan Nieboer, Theodorus H Van der Kwast, Geert van Leenders, Gleason Grade 4 Subtype Collaborative Group. Erasmus Medical Center, Rotterdam, Netherlands; University Health Network, Toronto, Canada.

Background: Gleason grade 4 prostate cancer represents a heterogeneous group of architectural growth patterns including ill-defined, fused, cribriform and glomeruloid. The aim of this study was to assess the interobserver reproducibility of individual Gleason grade 4 growth patterns.

Design: Twenty-three expert genitourinary pathologists assigned a Gleason grade (3, 4, or 5) to 60 high-magnification pictures of selected prostate cancer cases. The selection comprised comparable numbers of Gleason grade 3, 4 and 5 cases, including several purposely selected ambiguous cases. If a Gleason grade 4 was assigned, participants were requested to specify its growth pattern. We considered consensus to be reached when at least $80 \%$ of the participants agreed and a classification as "favored" when $60 \%-80 \%$ concurred.

Results: Gleason grade was consented in 47/60 (78\%) cases and favored in 9/60 (15\%); consensus on Gleason grade 4 was reached in 35 cases $(58 \%)$. When agreement on Gleason grade was less than $80 \%$, ill-defined $(6 / 13,46 \%)$ and fused $(7 / 13,54 \%)$ were the predominant alternatively assigned patterns. Among the 20 cases where at least 1 pathologist assigned ill-defined growth pattern none $(0 \%, 0 / 20)$ reached consensus. In the same way, consensus for fused was reached in 2\% (1/41), for cribriform $23 \%$ (7/30), and for glomeruloid 38\% (5/13). In 9/35 (26\%) consented Gleason grade 4 cases, participants disagreed on its growth pattern. These cases were characterized large epithelial proliferations with delicate intervening fibro-vascular cores and were alternatively assigned fused or cribriform growth pattern.

Conclusions: Consensus on Gleason grade 4 growth pattern was predominantly attained for cribriform and glomeruloid growth patterns and was low for ill-defined and fused morphology. These data indicate that Gleason grade 4 cribriform pattern, which has been associated with worse clinical outcome, is a reasonably reproducible marker in prostate cancer.

\section{Disease-Specific Survival of Patients with Invasive Cribriform and} Intraductal Prostate Cancer at Diagnostic Biopsy

Charlotte Kweldam, Intan Kummerlin, Daan Nieboer, Esther Verhoef, Ewout Steyerberg, Theodorus H Van der Kwast, Monique Roobol, Geert van Leenders. Erasmus Medical Center, Rotterdam, Netherlands; University Health Network, Toronto, Canada.

Background: Recent studies have shown that invasive cribriform and intraductal carcinoma are adverse prognostic factors in radical prostatectomies. To affect clinical decision-making, the added value of both pathological features in pre-treatment diagnostic biopsies needs to be assessed. We aimed to determine the prognostic value of invasive cribriform and intraductal carcinoma in diagnostic biopsies of prostate cancer patients on time to disease-specific death.

Design: We reviewed all diagnostic biopsies from 1031 patients included in the first screening round of the European Randomized Study of Screening for Prostate Cancer (ERSPC). Each biopsy was scored for modified Gleason score, presence of intraductal carcinoma, tumor percentage and individual Gleason grade 4 and 5 growth patterns. Patients who either had invasive cribriform growth or intraductal carcinoma were categorized as CR/IDC+. The primary outcome was disease-specific survival, which had been evaluated by an independent cause-of-death committee.

Results: The median follow-up was 13 years (IQR 9.4-16). After pathologic revision, 486 patients had Gleason score $6(47 \%)$ and 545 had 7 or higher $(53 \%)$. The 15 -year disease-specific-survival probabilities were $99 \%$ in Gleason score $6(n=486), 94 \%$ in CR/ IDC- $\geq 7(n=356)$ and $67 \%$ in $C R / I D C+\geq 7(n=189)$. In a multivariable cox regression analysis the following parameters predicted adverse outcome: Gleason score 8-10 (HR $3.1,95 \% \mathrm{CI} 1.1-8.3, P=.03$ ), CR/IDC+ status (HR 2.8, 95\% CI 1.5-5.2, $P<.001)$, PSA at diagnosis (HR 1.2 per doubling level, $95 \%$ CI 1.0-1.5, $P=.03$ ), and number of positive biopsy cores (HR 1.3, 95\% CI 1.1-1.5, $P=.004$ ).
Conclusions: Biopsy CR/IDC status is an independent predictor of disease-specific survival in prostate cancer patients. This may offer new opportunities for improving clinical-decision making, particularly in patients with Gleason score 7 prostate cancer.

973 Loss of Expression of INSL3 by immunohistochemistry Predicts Leydig Cell Tumor

Nelli Lakis, Zakaria Grada, Kara A Lombardo, Shamlal Mangray, Andres Matoso. Rhode Island Hospital/Brown University, Providence, RI.

Background: Insulin-like 3 (INSL3) is a hormone member of the relaxin-insulin-like family of peptide factors. It is almost exclusively produced by Leydig cells (LCs) within the testis and leads to physiological testicular descent during embryonic development. We aimed to investigate the expression of INSL3 by immunohistochemistry (IHC) in normal LCs, in Leydig cell hyperplasia (LCH) and in Leydig cell tumor (LCT) Design: IHC for INSL3 was analyzed in 16 orchiectomy specimens using a rabbit polyclonal antibody (Phoenix Pharmaceuticals, H-035-27; 1:2000 dilution). Results were interpreted using a combined score of extent and intensity on a scale of $0-3$ for percentage of cells and 0-3 for intensity. A combined score (CS) of $0-1=$ negative; 2 $=$ equivocal; $\geq 3$ = positive.

Results: The mean age of patients with LCTs was 41 (range 11-71). The average size of the tumor was $1.9 \mathrm{~cm}(0.4-3.9 \mathrm{~cm})$. The cases of LCH were a secondary finding in cases of malignant germ cell tumor $(n=5)$. Normally distributed LCs showed strong immunostaining in the cytoplasm in all cases and served as an internal control $(16 / 16$, $100 \%)$. Nine of ten $(90 \%)$ of the LCTs were completely negative and one case was equivocal. All 5 cases $(100 \%)$ of $\mathrm{LCH}$ were positive, including in areas of small nodule formation (Figure 1).

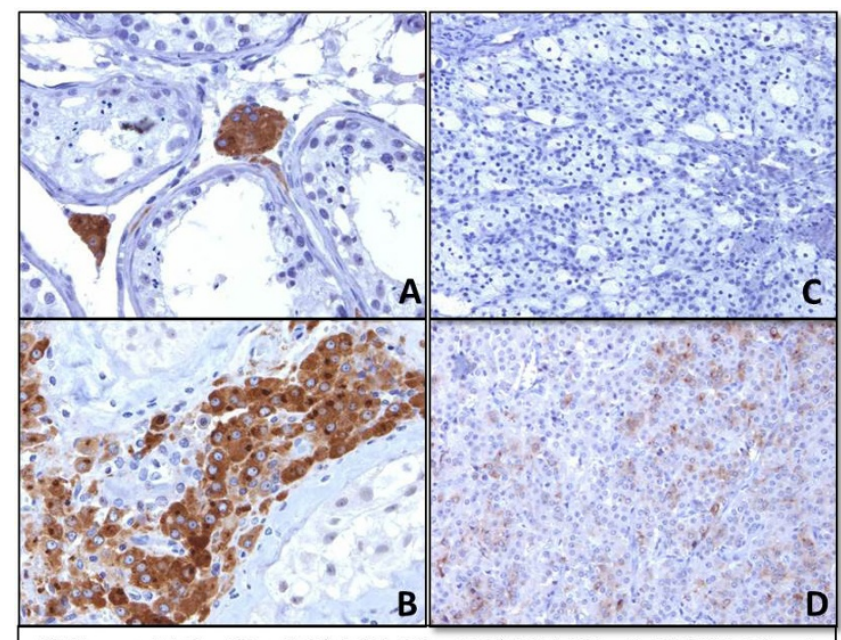

A) Normal LCS, CS = 3 B) LCH, CS = 3 C) LCT, CS = 0 D) LCT, CS = 1

Other sex cord stromal tumors tested were consistently negative including Sertoli cell tumor/nodules $(\mathrm{n}=4)$, fibrothecoma $(\mathrm{n}=1)$ and granulosa cell tumor $(\mathrm{n}=1)$.

Conclusions: INSL3 is a reliable marker of normal LCs. Its expression is retained in LCH but lost in LCTs. INSL3 IHC could be helpful to highlight Leydig cells in cases where it is difficult to identify them (i.e. small testicular biopsies performed for infertility workup) and to help in the differential diagnosis between florid LCH and LCT. While its role in tumorigenesis is largely unknown, its consistent underexpression in LCT suggests it could have a tumor suppressor role.

974 Expression of the Glucocorticoid Receptor in Prostatic Adenocarcinoma: An Immunohistochemical and Clinicopathologic Study Nelli Lakis, Andres Matoso, Shamlal Mangray, Murray B Resnick, Evgeny Yakirevich. Rhode Island Hospital/Brown University, Providence, RI

Background: Glucocorticoid receptors (GR) mediate the action of steroid hormones in a variety of tissues, including the prostate. Synthetic GR agonists or glucocorticoids (GCs), including prednisone, hydrocortisone and dexamethasone are used for the treatment of hormone refractory prostate cancer (HRPC) with response achieved in approximately $20 \%$ of patients. The role of GCs and GR expression in localized prostate cancer and cancer progression is unknown. The aim of this study was to evaluate GR expression by immunohistochemistry (IHC) in prostatic adenocarcinoma (AC) and correlate it with clinicopathologic characteristics.

Design: Immunohistochemical staining for GR was analyzed in 183 radical prostatectomy microarrayed specimens using a rabbit polyclonal antibody PA1-511A against GR (Affinity Bioreagents Golden, CO) at 1:400. Immunoreactivity was assessed according to a combined score of the extent and intensity on a scale of 1-4 for percentage of cells stained and 1-3 for intensity of staining. A total score of $\geq 5$ was considered strong, while $\leq 4$ was considered weak. The associations between GR expression, Gleason score, pathologic stage and survival were analyzed.

Results: Strong nuclear GR expression was localized to the nuclei of non-neoplastic glandular basal and luminal cells, and benign stromal cells. In the prostatic AC cases the staining pattern was similar to that in the non-neoplastic prostate with predominant nuclear GR localization. GR expression in tumor cells was found in $56 \%$ of cases with a distribution shown in Table 1 . 


\begin{tabular}{|l|l|l|l|l|}
\hline \multicolumn{5}{|l|}{ Table 1. GR Expression in Prostatic AC } \\
\hline & Cases (\%) & Negative (\%) & Weak (\%) & Strong (\%) \\
\hline Gleason $\leq 6$ & $99(54)$ & $49(50)$ & $29(29)$ & $21(21)$ \\
\hline Gleason $\geq 7$ & $84(46)$ & $29(34)$ & $25(30)$ & $30(36)$ \\
\hline Stage $\leq$ T2 & $148(81)$ & $61(41)$ & $41(28)$ & $46(31)$ \\
\hline Stage $\geq T 3$ & $35(19)$ & $13(36)$ & $11(32)$ & $11(32)$ \\
\hline
\end{tabular}

GR expression was higher in cases of Gleason $\geq 7 \mathrm{vs}$. Gleason $\leq 6(\mathrm{p}=0.05)$. There was no association between GR expression and pathologic stage. There was no difference in overall survival between GR positive vs. GR negative patients; however, disease free survival at 10 years was higher in GR negative patients ( $62 \%$ vs. $40 \%, \mathrm{p}=0.03$ ). Conclusions: This study is the first to examine GR expression in prostatic AC. GR expression is associated with higher Gleason score and lower disease free survival. This data supports a role for GR activity in promoting prostate cancer progression and invites further evaluation of GR as a potential therapeutic target in prostatic AC.

975 Prevalence, Distribution, and Viral Loads of 15 High-Risk HPV Types in Squamous Cell Carcinoma of the Scrotum

Marcos Lepe, M Ruhul Quddus, Pradip Manna, Jonathan I Epstein, Andres Matoso. Rhode Island Hospital and Brown University, Providence, RI; Women and Infant's Hospital and Brown University, Providence, RI; Johns Hopkins Hospital, Baltimore, MD; Physicians Reference Laboratory, LLC, Overland Park, KS; Brown University, Providence, RI.

Background: Squamous cell carcinoma (SCC) of the scrotum is associated with infection by high-risk human papilloma virus (HR-HPV) in a subset of patients. Which subtype(s) of HR-HPV are involved in this neoplasm is unknown.

Design: Paraffin embedded tissue samples from 27 cases of SCC of the scrotum were retrieved and all 15 subtypes of HR-HPV and their viral load were detected using multiplex real-time PCR method. The results were correlated with the histopathologic and clinical features.

Results: HR-HPV were identified in 15/27 (55\%) of the cases, including HPV16 ( $\mathrm{n}=8$ ), HPV35 ( $=7)$, HPV31 $(n=5)$, HPV33 ( $=4)$, HPV59 $(n=4)$, HPV18 $(n=2)$, HPV51 $(\mathrm{n}=2), \operatorname{HPV} 39(\mathrm{n}=1), \operatorname{HPV} 56(\mathrm{n}=1), \operatorname{HPV} 82(\mathrm{n}=1)$. The average viral loads normalized to 1,000 cells was 23,266 for HPV16; 35 for HPV18; 100,350,749 for HPV31; 406 for HPV33; 179,514 for HPV 35; 35 for HPV51; 1 for HPV39; 7 for HPV56; 828 for HPV59; and 6,734 for HPV82. Both HPV types 39 and 56 were detected but their levels were below the limit of quantification. All cases were negative for HPV45, HPV52, HPV58, HPV68 and HPV73. In-situ carcinomas had higher viral loads than invasive (50M vs. $2 \mathrm{M}$ in average). The average age of HPV positive and negative cases was similar; 55 (range 39-74) and 51 (range 39-63) respectively. Seven specimens were positive for multiple subtypes. 5/11 (45\%) case of invasive carcinoma were positive for HR-HPV versus 10/16 (63\%) of in-situ carcinomas. The highest proportion of HR-HPV positive cases was seen in basaloid type $(6 / 7 ; 86 \%)$, followed by warty $(3 / 4 ; 75 \%)$ and usual type $(6 / 16 ; 38 \%) .7 / 15$ (47\%) of the HPV positive cases were also positive for p16 by immunohistochemistry and 5/15 (33\%) were positive by FISH.

Conclusions: Similar to SCC of the vulva and penis, the most frequently HPV-positive tumors are basaloid and warty types. However, a smaller proportion of SCC usual-type are also positive for HPV. Our results show that $33 \%$ of cases are associated with HPV subtypes other than 16 or 18 and, therefore, are not currently covered by commercially available vaccines. Additionally, $70 \%$ of HPV $16 / 18$ positive cases are also coinfected with other subtypes, which suggest the vaccines would not work in those cases either.

976 A New AR-V7 Rabbit Monoclonal Antibody for Imunohistochemical Application

Aihua Li, Yuekai Zhang, Shihua Sun, Jason Law, Hongyang Pan, Jackie Chan, Daniel NavaRodrigues, Stephen Plymate, Johann S De Bono, Taiying Chen. Epitomics - an Abcam ${ }^{\circledR}$ Company, Burlingame, CA; University of Washington, Seattle, WA; Royal Cancer Hospital, London, United Kingdom.

Background: An underlying mechanism of failed response of metastatic castrationresistant prostate cancer (mCRPC) to enzalutamide and abiraterone treatment has been uncovered. A splice variant of androgen receptor protein AR-V7 (also known as AR3) has been attributed to the failure of MCRPC endocrine treatment. Therefore, an assay for AR-V7 protein in tissue by or circulating tumor cells is of critical importance. We report here a newly developed AR-V7 rabbit monoclonal antibody, clone EP343, and assessment of its utility for detection of AR-V7 expression.

Design: Rabbits were immunized with AR-V7 peptide. Hybridomas were generated using RabMAb technology. Antibodies were characterized by ELISA, WB using human cancer cell lines and immunohistochemistry (IHC) utilizing formalin fixed and paraffin embedded (FFPE) human normal, tumor tissue microarrays (TMA) and prostate cancer (PC) xenograft tissues. The correlation between IHC and mRNA level in xenograft tissues was also analyzed.

Results: An 80kDa AR-V7 protein was detected by WB in 22RV1 (AR-V7+) cells and CRPC tissue but not in DU-145 (AR-) PC cells. AR negative PC-3 cells also showed positive signal but no AR-V7 mRNA can be detected in these cells nor can the signal be decreased by siRNA specific for AR-V7. Tumor cell lines of the colon, breast, liver and glioblastoma tested were negative. IHC analysis of TMAs comprising 28 types of normal tissues and 16 types of tumor tissues was negative. Additional TMAs for 8 types of tumor tissues showed that AR-V7 was negative in tumors of the brain, stomach, breast, ovary, germ cell and lymphomas. Specific nuclear staining was found in 2 of 10 PCs, 1 of 56 colon carcinomas. Xenograft tumors from AR-V7+ PC cells VCAP and LNCaP 95 showed strong nuclear staining in tumor cells. The positive staining is correlated with the mRNA level of AR-V7.

Conclusions: RabMAb anti-AR-V7, clone EP343 is specific and sensitive in the detection of target AR-V7 proteins in FFPE tissues. Its WB and IHC reactivity in PC-3 and colon carcinoma respectively warrant further investigations. EP343 may be a potential tool for IHC assessment of AR-V7 expression in CRPC. Additional studies using large number of CRPC are needed to further determine the clinical utility of this antibody.

\section{Diagnostic Accuracy of Subpatterns of Gleason Pattern 4 Prostate} Cancer Morphological Subpatterns

Jianhong Li, Rajal Shah, Ali Amin, Ritu Bhalla, Kasturi Das, Fang-Ming Deng, Peng Lee, Andres Matoso, Jonathan Melamed, Savvas Mendrinos, Wei Tian, Oksana Yaskiv, Ming Zhou. New York University, New York, NY; Miracal Life Sciences, Irving, TX; Brown University, Providence, RI; Louisiana State University, New Orleans, LA; North Shore LIJ, Lake Success, NY.

Background: Gleason pattern (GP) 4 prostate cancer (PCa) signals aggressive disease. It has several morphological subpatterns, including poorly formed glands $(\mathrm{P})$, fused glands (F), glomeruloid structures $(\mathrm{G})$ and cribriform glands $(\mathrm{C})$. Recent studies found that $\mathrm{C}$ subpattern is associated with a worse prognosis than other subpatterns. It is therefore important to correctly identify GP4 subpatterns. We investigated the diagnostic accuracy of GP4 subpatterns among genitourinary (GU) pathologists

Design: 47 glass slides containing 55 biopsy cores of various amounts of GP4 PCa were distributed to $12 \mathrm{GU}$ pathologists who were asked to identify all GP4 subpatterns and the most common subpattern in each core. A subpattern is considered present or absent by consensus when $\geq 75 \%$ (9) or $\leq 25 \%$ (3) participants recognized it in a core. A subpattern is considered the most common pattern when indicated by $\geq 9$ participants. Diagnostic accuracy $=$ (correctly identified cores with and without a subpattern)/total cores.

Results: Results: P, F, C and G subpatterns are identified in $32(58 \%), 21(38 \%), 13$ $(24 \%)$ and $8(15 \%)$ and of 55 cores. 1, 2, 3 and 4 subpatterns were identified in 32 $(58 \%), 14(25 \%), 2(4 \%)$ and $1(2 \%)$ of 55 cores. In $6(11 \%)$ there was no consensus. Of 17 cores with $\geq 2$ subpatterns, $\mathrm{P}$ and $\mathrm{F}$ subpatterns were seen together in 16 (94\%) while $\mathrm{G}$ and $\mathrm{C}$ subpatterns together in $2(12 \%)$. The diagnostic accuracy for $\mathrm{P}, \mathrm{F}, \mathrm{C}$ and $\mathrm{G}$ were $61.2 \%, 36.1 \%, 79.4 \%$ and $77.1 \%$, respectively (p values for $\mathrm{P} / \mathrm{F}, \mathrm{P} / \mathrm{G}$ and $\mathrm{G} / \mathrm{C}$ were $<0.001,<0.001$ and 0.317 ). P, F, C and G were the most common subpattern in 18 (33\%), $6(11 \%), 9(16 \%)$ and $1(2 \%)$, respectively. $20(36 \%)$ cores had no consensus regarding the most common subpattern. In these cores, the 2 most common subpatterns were $\mathrm{P}$ and $\mathrm{F}$ in 17 (85\%), $\mathrm{P}$ and $\mathrm{G}$ in $1(5 \%), \mathrm{F}$ and $\mathrm{G}$ in $1(5 \%)$ and $\mathrm{P}$ and $\mathrm{C}$ in $1(5 \%)$ core, suggesting that $\mathrm{P}$ and $\mathrm{F}$ subpatterns were often mistaken for each other. Conclusions: GU pathologists can reliably identify $\mathrm{C}$ and $\mathrm{G}$ subpatterns with diagnostic accuracy $>75 \%$ in prostate biopsies. However, the diagnostic accuracy for $\mathrm{P}$ and $\mathrm{F}$ is significantly lower. F subpattern has the worst diagnostic accuracy at $36 \%$, indicating a need for better definition of these 2 subpatterns.

978 High-Grade Prostatic Intraepithelial Neoplasia with Adjacent Small Atypical Glands (PINATYP): Reappraisal of Diagnostic Criteria

Jianhong Li, Fang Bu, Fang-Ming Deng, Peng Lee, Jonathan Melamed, Rajal Shah, Ming Zhou. New York University, New York, NY; Miracal Life Sciences, Irving, TX. Background: High-grade prostatic intraepithelial neoplasia (HGPIN) with adjacent small atypical glands (PINATYP) may represent minute focus of invasive prostate cancer (PCa) or budding/tangentially sectioned glands off adjacent HGPIN. Such distinction, although important, is often difficult in biopsy samples. We studied the morphological features that may aid the differential diagnosis of the two entities.

Design: We studied 50 foci of HGPIN not associated with PCa (non-PCa foci) and 47 foci of HGPIN intermixed with PCa (PCa foci) in 43 radical prostatectomies. A cocktail of p63, K903 and AMACR were performed on all chosen areas. The following parameters were measured for both groups: number of HGPIN and adjacent atypical glands (ATYP), number of ATYP with and without basal cells, distance of ATYP from the HGPIN glands $(<0.1 \mathrm{~mm}$ vs. $>0.1 \mathrm{~mm})$ and concordance of basal cell marker and AMACR expression between ATYP and adjacent HGPIN. Discordance in basal cell staining is defined as ATYP without basal cells adjacent to HGPIN glands with complete or near complete basal cell lining.

Results: The ratio of ATYP/HGPIN glands was significantly higher in PCa foci than in non-PCa foci ( 1.64 vs. $0.37, \mathrm{p}<0.001)$. ATYP in non-PCa foci were more likely to be very close to and within $0.1 \mathrm{~mm}$ of HGPIN compared to PCa foci $(97 \%$ vs. $47 \%$, $\mathrm{p}<0.001)$. ATYP in non-PCa foci is more likely to have basal cell lining than ATYP in PCa foci $(97 \%$ vs. $23 \%$ p $<0.001)$. Only 16 ATYP (out of 1346 HGPIN, 0.01 ATYP per HGPIN) in non-PCa foci had no basal cell lining, compared with $1239(77 \%)$ in $\mathrm{PCa}$ foci. Of 16 ATYP without basal cells in non-PCa foci, 3 (19\%) had basal cell marker and AMACR expression discordant with adjacent HGPIN. In contrast, of 1239 ATYP without basal cells in PCa foci, 943 (77\%) had basal cell marker and $495(40 \%)$ had AMACR expression discordant with HGPIN.

Conclusions: ATYP adjacent to HGPIN in non-PCa foci are more likely to preserve basal cells staining and significantly more likely to have basal cell and AMACR expression concordant with adjacent HGPIN. Basal cell negative ATYP adjacent to HGPIN is exceedingly rare in the absence of concomitant PCa. Our results suggest that PINATYP in prostate biopsy without $\mathrm{PCa}$, especially when there are several ATYP glands with basal cell and AMACR expression discordant with adjacent HGPIN, likely represents early invasive $\mathrm{PCa}$ and should prompt more aggressive workup. 
979 Quantifying Gleason Pattern 4 Prostate Cancer in Prostate Needle Biopsy: An Interobserver Reproducibility Study

Jianhong Li, Jianbo Li, Ali Amin, Ritu Bhalla, Kasturi Das, Fang-Ming Deng, Peng Lee, Andres Matoso, Jonathan Melamed, Savvas Mendrinos, Wei Tian, Oksana Yaskiv, Rajal Shah, Ming Zhou. New York University, New York, NY; Cleverland Clinic, Cleveland, $\mathrm{OH}$; Louisiana State University, New Orleans, LA; Northshore LIJ Health System, Lake Success, NY; Brown University, Providence, RI; Miracle Life Sciences, Irving, TX. Background: Gleason pattern (GP) 4 indicates potentially clinically significant prostate cancer (PCa). Furthermore, the quantity of GP4 cancer significantly impacts the prognosis. Recently, ISUP recommended that percentage of GP4 be included in the biopsy report. However, the interobserver reproducibility of quantifying GP4 among pathologists is unknown. It is also not known if the subpatterns of GP4 affect how pathologists quantify GP4

Design: 47 glass slides containing 55 biopsy cores of various amounts of GP4 PCa were distributed to $12 \mathrm{GU}$ pathologists who were asked to quantify percentage of GP4 and identify the most common GP4 subpattern (poorly formed glands [P], fused glands [F], cribriform $[\mathrm{C}]$ and glomeruloid $[\mathrm{G}]$ ) in each core. A subpattern is considered the most common pattern by consensus when identified by $\geq 75 \%$ (9) participants.

Results: 35 biopsy cores contained Gleason score (GS) $3+4=7$ PCa while 20 contained GS4+3=7 PCa. The mean PCa length was $5.4(0.6-13) \mathrm{mm}$. P, F, C and G was the most common subpattern in $18(33 \%), 6(11 \%), 9(16 \%)$ and $1(2 \%)$ cores, respectively. There was no consensus regarding the most common subpattern in $20(36 \%)$ cores. The interobserver reproducibility for 12 pathologists to quantify 55 biopsy cores was fair (kappa $=0.57$ ). These 55 cores were divided into 3 groups based on the PCa length, group $1(\leq 2 \mathrm{~mm})$, group $2(2.1-5 \mathrm{~mm})$ and group $3(>5 \mathrm{~mm})$. The kappa values for these 3 groups were $0.51,0.50$ and 0.66 , respectively ( $\mathrm{p}$ value was $>0.05$ for group 1 vs 2 and $<0.05$ for group 2 vs 3 ). These 55 cores were also divided into 4 groups according to their most common subpattern (P, F, [C or G], no consensus). The kappa values for these 4 groups were 0.43 . $0.57,0.74$ and 0.57 , respectively ( $\mathrm{p}$ value $<0.05$ for $\mathrm{P}$ vs $\mathrm{F}$ and $\mathrm{F}$ vs [C or G]). 11 of 12 participants based GP4 percentage quantification on the areas of GP4 vs total cancer area, and one participant based the quantification on the length of GP4 vs total cancer length.

Conclusions: The reproducibility of quantifying percentage of GP4 PCa is only fair (kappa $=0.57$ ) among GU pathologists. The reproducibility is significantly lower for small focus of cancer and cancer with predominant poorly formed and fused gland pattern. These findings argue for more training and education for pathologists before reporting $\% \mathrm{GP} 4$ becomes mandatory.

980 Pediatric Cystic Nephromas Are Morphologically, Immunohistochemically, and Genetically Distinct from Most Adult Cystic Nephroma/Mixed Epithelial Stromal Tumor (MEST) Family Lesions

Yunjie LI, Bruce R Pawel, Dana A Hill, George J Netto, Jonathan I Epstein, Pedram Argani. Johns Hopkins University School of Medicine, Baltimore, MD; Children's Hospital of Pennsylvania, Philadelphia, PA; Children's National Medical Center, Washington, DC.

Background: The term cystic nephroma (CN) has historically been applied to both pediatric and adult cystic renal neoplasms. In the 2016 World Health Organization classification, pediatric $\mathrm{CN}$ is considered a distinct entity associated with DICERI mutations, while adult $\mathrm{CN}$ are considered part of the mixed epithelial stromal tumor (MEST) family of neoplasms. A detailed comparison of these two lesions has not been performed.

Design: We reviewed cases classified as pediatric $\mathrm{CN}$ and adult MEST/CN from two institutions. Six cases originally classified as adult MEST/CN were excluded and reclassified as tubulocystic renal cell carcinoma (RCC) (1 case), multilocular cystic RCC (2 cases) and smooth muscle and adenoma-like renal tumor (SMART) (3 cases). One case originally classified as pediatric $\mathrm{CN}$ was excluded and reclassified as localized segmental polycystic kidney disease. This left 12 adult MEST/CN cases and 7 pediatric $\mathrm{CN}$ cases for study. On all cases, immunohistochemistry (IHC) for estrogen receptor (ER) and inhibin was performed, and mutation status of DICERI was assessed when possible.

Results: Comparison of Pediatric CN and Adult MEST/CN

\begin{tabular}{|l|l|l|l|}
\hline & Pediatric CN & Adult MEST/CN & p Value \\
\hline Mean Age (years) & 1.4 & 52.2 & $<0.00001$ \\
\hline Gender (M:F) & $6: 1$ & $0: 12$ & 0.0003 \\
\hline Subepithelial Cellular Stroma & $6 / 7(86 \%)$ & $11 / 12(92 \%)$ & $\mathrm{NS}$ \\
\hline Wavy or Ropy Collagen & $0 / 7(0 \%)$ & $7 / 12(58 \%)$ & 0.02 \\
\hline ER IHC Labeling & $7 / 7(100 \%)$ & $12 / 12(100 \%)$ & $\mathrm{NS}$ \\
\hline Inhibin IHC Labeling & $0 / 7(0 \%)$ & $10 / 12(83 \%)$ & 0.0007 \\
\hline DICER1 Mutations & $6 / 7(86 \%)$ & $1 / 10(10 \%)$ & 0.004 \\
\hline
\end{tabular}

Conclusions: While pediatric $\mathrm{CN}$ may show cellular subepithelial condensations that mimic ovarian stroma, true ovarian-like stroma with ropy collagen and inhibin labeling is typically absent in pediatric $\mathrm{CN}$ and present in most adult MEST/CN family tumors. ER labeling in pediatric $\mathrm{CN}$ may reflect induction associated with cyst formation as previously been described in response to obstruction (Mod Pathol 2008; 21:60-5). The absence of DICER1 mutations in most adult MEST/CN family tumors corroborates morphologic and IHC differences supporting the concept that most adult MEST/CN and pediatric $\mathrm{CN}$ represent distinctive neoplastic entities. A small subset of lesions currently classified as adult MEST/CN may be genetically related to pediatric CN.
981 Sunitinib Treatment Induces the Formation of Stem Cell-Like Cancer Cells in Clear Cell Renal Cell Carcinoma

Zsuzsanna Lichner, Henriett Butz, Roy Nofech-Mozes, Sara Riad, Andras Kapus, George M Yousef. Li Ka Shing Knowledge Institute, St Michael's Hospital, Toronto, ON, Canada; University of Toronto, Toronto, ON, Canada.

Background: Sunitinib (SU), a multi-receptor tyrosine kinase inhibitor, is the first line treatment for advanced renal cell carcinoma (RCC). SU prolongs PFS by 6 months; however, patients eventually develop treatment resistance by a largely unknown mechanism. Here we investigated potential mechanisms of SU resistance in vitro and using an animal model.

Design: In vitro: $\mathrm{RCC}$ cell lines were treated with SU and cell proliferation was used as measure of sensitive/resistant status. In vivo: RCC cell lines were xenografted and tumor bearing mice were randomized to vehicle treated, SU sensitive and SU resistant groups $(\mathrm{N}=25)$. Gene- and miRNA expression was assessed by NGS on Illumina MiSeq platform and by TaqMan miRNA arrays. Expression was validated by qPCR and by ICC. Tumorigenicity was assessed by limiting dilution assay (LDA).

Results: In vitro: RCC cell lines were treated with SU for 16-20 weeks. In line with the in vivo expression data, an increase of stem cell marker (OCT4, KLF4, NANOG) and cancer stem cell marker (LGR4, LGR5, DNAJB9, NRCAM, ENG) expression was observed. SU treatment initiated the formation of RCC spheres with stem cell characteristics in the drug sensitive phase, in all cell lines. These spheres expressed high level of stem cell and cancer stem cell markers and were highly clonogenic, indicating potent self-renewal. In vivo: miRNA expression and transcriptome of vehicle treated, SU sensitive and SU resistant xenografts were compared. Differentially expressed genes were grouped into 7 expression patterns. Significant functional categories included stem cell, cell differentiation, development, proliferation, cell-cell and cell-ECM interactions and metabolic processes. Significant signaling pathways were activation of hypoxic pathways, BMP signaling and canonical and non-canonical WNT signaling. LDA revealed that 50 sphere derived ACHN cells were enough to generate a xenograft, indicating high self-renewal ability and tumorigenicity of these cells.

Conclusions: Our results indicate that RCC cells gain stem cell characteristics during SU treatment. We point out a novel mechanism which potentially is able to contribute to the development of SU resistance in advanced RCC.

982 Using Next Generation Sequencing and Fluorescence In Situ Hybridization to Identify a Hemizygous Deletion of the RB1 Gene in Chromophobe Renal Cell Carcinoma: Its Potential Role in Distinguishing Chromophobe Renal Cell Carcinoma from Renal Oncocytoma

Qingqing Liu, Kristine M Cornejo, Liang Cheng, Lloyd Hutchinson, Keith Tomaszewicz, Ediz F Cosar, Bruce A Woda, Zhong Jiang. University of Massachusetts Medical School and UMass Memorial Medical Center, Worcester, MA; Indiana University School of Medicine, Indianapolis, IN.

Background: The histopathological distinction between chromophobe renal cell carcinoma (ChRCC) and renal oncocytoma (RO) may be very challenging as they share overlapping morphological, immunohistochemical and ultrastructural features. The aim of this study was to use next generation sequencing (NGS) to identify a genetic marker that is specifically expressed in $\mathrm{ChRCC}$, but not in RO, thus aiding in the diagnosis of ChRCC.

Design: A total of 20 cases $(\mathrm{ChRCC}, \mathrm{n}=11 ; \mathrm{RO}, \mathrm{n}=9$ ) were retrieved from the surgical pathology files of a tertiary medical center. NGS of 50 cancer "hotspot" gene mutations and gene copy number analysis using the Ampliseq Cancer Hotspot Panel v2 was performed in both ChRCC and RO. Matched normal tissue from the same specimen was used for comparison. The gene copy number data was confirmed by fluorescence in situ hybridization (FISH).

Results: The 11 ChRCC cases were from 3 female and 8 male patients with a mean age of 50.8 (28-75) years. The 9 RO cases were from 2 female and 7 male patients with a mean age of 64.3 (41-84) years. No "hotspot" mutations in cancer-critical genes were identified in either ChRCC or RO. Gene copy number analysis using NGS revealed a hemizygous deletion of tumor suppressor gene RB1 in ChRCC and not RO. Subsequent FISH analysis confirmed the hemizygous deletion of the RB1 gene in 6 of 11 ChRCC $(55 \%)$, but not in any RO $(0 / 9 ; 0 \%)$. The sensitivity and specificity of hemizygous RB1 deletion in differentiating ChRCC from RO is $55 \%$ and $100 \%$, respectively.

Conclusions: In summary, a hemizygous deletion of RB1 by FISH can be used to distinguish ChRCC from RO. The high degree of specificity of RB1 gene deletion for ChRCC in formalin-fixed, paraffin-embedded tissue sections suggests that it may be of diagnostic utility in distinguishing these two entities in daily clinical practice.

983 Mutational Analysis of Prostate Cancer Using Next Generation Cancer Hotspot Panel

Zach Liu, Ming Zhou, Herber Lepor, Roberto Zoino, Gaurav Rajoria, Szczepan Klimek. Histopathology Services, Ramsey, NJ; New York University Medical Center, New York City, NY.

Background: Prostate cancer (PCA) is a clinically and genetically heterogeneous disease. Understanding the genetic abnormalities is necessary to better determine the risk of disease progression and to identify potential targets for precision therapy. Many studies were performed on high grade and advanced stage cancer. We analyzed the mutational landscape in intermediate grade (Gleason score 7) PCA.

Design: 36 cases of PCA with Gleason score $3+4$ or $4+3$ or were selected from archives. The tumor areas were reviewed by pathologists. Formalin-fixed and paraffin embedded tumor was micro-dissected and subjected to the next-generation sequencing using the commercially available Ion Torrent Hotspot Cancer Panel from (ThermoFisher 
Scientific, Waltham, MA). For each gene, the minimum required coverage was 500 sequence reads. The minimum detectable mutant allele ratio was $\sim 5 \%$. This test is for gene mutation, not for copy number changes or translocations.

Results: 36 cases yielded interpretable sequencing data. 23 cases (64\%) showed at least one mutated gene. Of these 23 positive cases, 7 (19\% of total) had multiple gene mutations. The mutated genes include KRAS (2.8\%), TP53 (11.1\%), MLH1 (5.6\%), MET (8.3\%), KIT (22.2\%), IDH1 (2.8\%), JAK3 (5.6\%), JAK2 (2.8\%), BRAF (5.6\%), $\operatorname{ATM}(5.6 \%)$, STK11 (5.6\%), PIK3CA (2.8\%), APC (2.8\%). c-kit mutation at p.M541L was the most frequently mutated gene in our study $22.2 \%$ ). This mutation is identical to the mutations detected in chronic myeloid leukemia and GIST.

Conclusions: Our data showed $64 \%$ of Gleason score 7 PCA harbors mutations in at least one gene in the Ion Torrent Hotspot Cancer Panel. Most of these mutations have been significant in other cancers and their significance is at present unknown in PCA and deserves further investigation. A surprisingly high frequency of c-kit mutation $(22.2 \%)$ was detected in our cohort, much higher than the minor allele frequency in normal population (MAF: 0.06). Further studies are warranted.

984 Clinical Validation of AR-V7 Detection in Circulating Tumor Cells from Castration-Resistant Prostate Cancer Patients

Parvez M Lokhandwala, Stacy Riel, Christopher D Gocke, Ming-Tseh Lin, Gang Zheng, Emmanuel S Antonarakis, Jun Luo, James R Eshleman. Johns Hopkins University School of Medicine, Baltimore, MD

Background: Castration-resistant prostate cancer (CRPC) patients are treated with agents that target the ligand binding domain of the androgen receptor (AR) (e.g. enzalutamide and abiraterone). Approximately $20-40 \%$ of such patients have poor clinical response to these agents, and even patients who initially respond can acquire secondary resistance. Androgen receptor splice variant 7 (AR-V7) lacks the ligandbinding domain. Detection of AR-V7 mRNA in the circulating tumor cells (CTCs) of such patients is associated with resistance to AR-targeting agents but continued sensitivity to taxane therapy. We describe validation of the first AR-V7 laboratorydeveloped test in a CLIA-certified clinical laboratory.

Design: CTCs from blood of advanced prostate cancer patients were isolated by immunomagnetic enrichment. The mRNA from enriched cells was isolated using Oligo- $\mathrm{d}(\mathrm{T})_{25}$-coated beads and reverse transcribed into cDNA. Q-PCR amplification of two house-keeping genes (ACTB and GAPDH), three genes to determine the presence of CTCs (PSMA, PSA and AR-full length (AR-FL)), and AR-V7 was performed. The following specimens were used for validation: a prostate cancer cell line expressing AR-V7 (LNCaP-95), 38 peripheral blood controls (14 males, 24 females), and 21 blood samples from men with CRPC

Results: The assay reproducibly detected the presence of five LnCAP-95 cells spiked into $5 \mathrm{ml}$ of control peripheral blood (or equivalent to an estimated $0.001 \mathrm{ng}$ of LNCaP-95 input RNA), demonstrating high analytic sensitivity. Multiple inter- and intra-run replicates of LNCaP-95 cell line yielded similar Ct values for all genes, showing high analytic precision. All 38 healthy donor samples were negative for AR-V7, but expressed both house-keeping genes, demonstrating high analytic specificity. All healthy donor samples were negative for PSA and PSMA; however, AR-FL was detected at very low levels $(\mathrm{Ct}$ value $>39)$ in 3 healthy donors -2 elderly females and 1 male $($ age $<40$ ). The diagnostic accuracy was confirmed by concurrent testing of 21 CRPC samples between the Luo research laboratory (that developed the test) and our clinical laboratory, which were concordant for AR-V7 (positive in 4 cases, negative in 17 cases). Conclusions: Our clinical assay detects CTC-derived AR-V7 with high analytic sensitivity, analytic precision, diagnostic specificity and diagnostic accuracy. It is currently in use for targeted therapy trials.

985 Pathologic Outcomes and Biochemical Recurrence (BCR) Free Survival in Men Younger Than 45 Years with Prostate Cancer (PCa) Treated with Robotic Radical Prostatectomy (RRP)

Zhichun Lu, Sean R Williamson, Mireva Diaz-Insua, Hans Stricker, Mani Menon, Nilesh $S$ Gupta. Henry Ford Hospital, Detroit, MI.

Background: PCa diagnosed in young men has not been adequately studied in the PSA screening era. There are conflicting studies regarding the behavior of PCa in young men. The aim of our study was to study the clinical and pathological characteristics of $\mathrm{PCa}$ in men younger than 45 years treated with RRP.

Design: Our study group comprised of 117 patients younger than 45 years, who underwent RRP between 2001-2013. We recorded clinical data from hospital records and grading/staging parameters from review of prostate needle biopsies (PNB) and RRP. Results: Age of our study population ranged from 33 to 45 (Mean:42) years. Clinical parameters are listed in table 2. Pre-operative PSA ranged from 0.3 to 22.4 (Mean: 5.78). PNB data was available on 81 pts, Gleason score (GS) 6 in 45 (56\%) cases, GS $3+4=7$ in $28(35 \%)$ cases, GS $4+3=7$ in $5(6 \%)$ cases, GS 8 in $1(1 \%)$ case and GS9/ GS10 in $2(2 \%)$ cases. Follow up period ranged from 1-123.7 (Mean: 38.3 ) months.

\begin{tabular}{|l|l|l|}
\hline Clinical parameters & Sub-categories & Number of patients (\%) \\
\hline \multirow{5}{*}{ Race } & Caucasian & $47(40)$ \\
\cline { 2 - 3 } & African American & $22(19)$ \\
\cline { 2 - 3 } & Unknown & $40(34)$ \\
\cline { 2 - 3 } & Other & $8(7)$ \\
\hline \multirow{5}{*}{ Family History } & Positive & $51(46)$ \\
\cline { 2 - 3 } & Negative & $45(38)$ \\
\hline & Not known & $21(18)$ \\
\hline \multirow{5}{*}{ BCR } & T1c & $85(73)$ \\
\cline { 2 - 3 } & T2a & $15(13)$ \\
\cline { 2 - 3 } & T2b & $6(5)$ \\
\cline { 2 - 3 } & T2c & $10(8)$ \\
\cline { 2 - 3 } & T3b & $1(1)$ \\
\hline & BCR positive & $10(9)$ \\
\cline { 2 - 3 } & BCR negative & $102(87)$ \\
\cline { 2 - 3 } & Lost to follow up & $5(4)$ \\
\hline
\end{tabular}

\begin{tabular}{|l|l|l|}
\hline RRP parameters & Sub-categories & Number of patients (\%) \\
\hline \multirow{5}{*}{ GS } & 6 & $48(42)$ \\
\cline { 2 - 3 } & $3+4=7$ & $54(46)$ \\
\cline { 2 - 3 } & $4+3=7$ & $7(6)$ \\
\cline { 2 - 3 } & 8 & $4(3)$ \\
\cline { 2 - 3 } & $9 / 10$ & $4(3)$ \\
\hline \multirow{5}{*}{ Tumor Volume } & $<5 \%$ & $41(35)$ \\
\cline { 2 - 3 } & $6-10 \%$ & $26(22)$ \\
\cline { 2 - 3 } & $11-20 \%$ & $29(25)$ \\
\cline { 2 - 3 } & $>20 \%$ & $21(18)$ \\
\hline \multirow{5}{*}{ Margins } & pT2 & $89(76)$ \\
\cline { 2 - 3 } & pT3a & $25(21)$ \\
\cline { 2 - 3 } & pT3b & $3(3)$ \\
\hline \multirow{5}{*}{ LN Stage } & Negative & $89(76)$ \\
\cline { 2 - 3 } & Focal positive & $13(11)$ \\
\cline { 2 - 3 } & Positive & $15(13)$ \\
\hline & No lymph nodes present & $30(26)$ \\
\cline { 2 - 3 } & Negative for metastasis & $85(73)$ \\
\cline { 2 - 3 } & Metastasis present & $2(2)$ \\
\hline
\end{tabular}

Conclusions: Young patients with PCa treated with RRP tend to present with lower grade and lower stage disease and show favorable disease-free outcome compared to $\mathrm{PCa}$ seen in older age group. We had high percentage of patients with positive family history of $\mathrm{PCa}$ but did not find significant difference in pathologic outcome and $\mathrm{BCR}$ rates in those patients compared to ones lacking family history of $\mathrm{PCa}$

\section{Amyloidosis of the Bladder and Association with Urothelial} Carcinoma: Report of 18 Cases

Daniel J Luthringer, Deepika Sirohi, Mahul B Amin. Cedars Sinai Medical Center, Los Angeles, CA.

Background: Some forms of amyloidosis are associated with neoplastic processes such as multiple myeloma, lymphoma and endocrine neoplasms including medullary thyroid carcinoma. Amyloid rarely occurs with other epithelial neoplasms. Amyloid deposits are uncommonly found in the bladder and rarely occur in relationship to urothelial carcinoma (UC), with only 2 reported cases. In the largest review of 31 cases of primary localized amyloidosis of the bladder, none had an association with UC. Herein, we report the characteristics of 18 cases of amyloidosis in the bladder, 9 of which were found in relationship to UC.

Design: With IRB approval, we searched departmental and personal consultation files (MBA) for cases of amyloidosis involving bladder tissue spanning years 2005 to 2015. Reports, slides, special studies, clinical records, \& laboratory results [i.e. serum electrophoresis (SPEP), mass spectrometry data, immunohistochemical (IHC) assays] were reviewed. The diagnosis of amyloid was made by Congo red stain showing applegreen birefringence on polarization microscopy and special studies in some cases.

Results: 18 cases of amyloid were identified in bladder specimens (12 biopsies, 4 transurethral resections, 2 cystoprostatectomies). Patient ages ranged from 50 to 102 yrs (mean 74.5) with a M:F of 5:1. Cases presented as mass lesions ( $\mathrm{n}=12)$, hematuria $(n=2) \&$ unspecified $(n=2)$. Congo red stain confirmed amyloid in all cases. Deposits were in the subepithelial stroma, in diffuse, nodular or perivascular distributions. IHC workup for subtyping done in 10 cases showed transthyretin ( $n=6)$, AL ( $n=2)$, Amyloid $\mathrm{P}(\mathrm{n}=1)$ \& undetermined $(\mathrm{n}=1)$ types. SPEP was negative in 5 cases. One case analyzed by mass spectrometry was AL type. 14 cases were classified as localized primary amyloidosis and 4 as secondary due to systemic amyloidosis. 9 cases had an associated UC; 4 low grade non-invasive papillary, 3 high grade non-invasive papillary \& 2 invasive high grade (into lamina propria \& muscularis propria). In UC cases, amyloid deposits were in the stroma immediately beneath or adjacent to the tumor, but never an integral part of the tumor.

Conclusions: Amyloidosis of the bladder rarely occurs and can be the manifestation of localized deposits or systemic disease. It frequently presents as a mucosal mass that can mimic UC. In half of cases, amyloid deposits are found in the stroma near 
various morphologies of UC. The relationship of amyloid in the bladder and UC, though likely not directly causal, appears to be a unique finding not frequently seen with other solid tumors.

\section{Unclassified Renal Cell Carcinomas with Low-Grade Oncocytic} Features: A Histopathologic Study with Outcome

Martin J Magers, Angela Wu, Lakshmi P Kunju. University of Michigan, Ann Arbor, MI. Background: Most unclassified renal cell carcinomas (URCCs) are aggressive and high-grade. However, a subset of URCC with low-grade features has been reported. We evaluated the clinico-pathologic features of low-grade URCC with oncocytic features (LGO-RCC).

Design: An electronic records search identified patients with LGO-RCC resected at our institution (2000-2015). Only cases with prominent low-grade oncocytic areas were included; typical URCCs with significant high-grade atypia including rhabdoid features were excluded. Multiple clinicopathologic parameters and immunohistochemistry were assessed

Results: Nineteen patients [mean 64 years, range 37-80; M:F 10:9; 10 partial and 9 radical nephrectomies; majority (18/19) with a solitary mass] were included. Tumors (mean $5.4 \mathrm{~cm}$, median $5.2 \mathrm{~cm}, 1-13 \mathrm{~cm}$ ) were predominantly pT1 $(68 \%)$ and rarely pT3a (16\%). Most (68\%) had a central scar and about half were mahogany-brown. Most contained small nests or tubules $(68 \%)$ within an edematous stroma (74\%) and/ or solid sheets of cells $(63 \%)$; a subset showed a large nested/pseudopapillary pattern (37\%). While all tumors had dense oncocytic cytoplasm, a subset had focal flocculent cytoplasm (42\%). Atypia was present including mild nuclear pleomorphism (100\%), irregular nuclear contours (68\%), hyperchromasia (47\%), prominent nucleoli $(32 \%)$, and degenerative atypia (47\%). Rare features included scattered clear cells $(16 \%)$, very focal koilocytic atypia $(16 \%)$, necrosis $(5 \%)$, sarcomatoid features $(5 \%)$, and increased mitoses (5\%). Most cases were positive for CK7 (12/19, focal), C-Kit (12/16, diffuse membranous), S100-A1 (8/8), PAX8 (5/5), and AMACR (2/3); vimentin was occasionally expressed (7/15). Based on overall architecture/nuclear features, four patterns were identified: oncocytoma-like $(8 / 19,42 \%)$, prominent tubules/cysts with intratubular secretions $(3 / 19,16 \%)$, prominent intracytoplasmic inclusions $(3 / 19$, $16 \%)$, and not otherwise specified $(5 / 19,26 \%)$. One patient developed metastases/ died of disease $(6.4 \mathrm{~cm}, \mathrm{pT} 1 \mathrm{~b})$, and one was lost to follow up; the remaining were disease free/died of other causes (mean and median follow-up time 19 and 12 months respectively, 0-70 months).

Conclusions: Most LGO-RCCs are relatively small, confined to the kidney, and the vast majority behave in an indolent fashion. Many $(42 \%)$ are architecturally oncocytomalike with cytologic atypia beyond the threshold of typical oncocytoma. LGO-RCC represents a small subset of URCC which should be distinguished from typical URCC.

\section{Spectrum of PD-L1 Expression in Penile Squamous Cell Carcinoma (SqCC)}

Martin J Magers, Aaron Udager, Tzu-Ying Liu, Andrew McDaniel, Stephanie L Skala, Todd Morgan, Ganesh Palapattu, Alon Z Weizer, Tina Fields, Ajjai Alva, Jeffrey S Montgomery, Arul M Chinnaiyan, Scott A Tomlins, Hui Jiang, Rohit Mehra. University of Michigan Health System, Ann Arbor, MI.

Background: $\mathrm{SqCC}$ is the most common primary penile neoplasm. Treatment options for low-stage, localized tumors is primarily surgical resection with or without radiation therapy, while high-stage, locally advanced tumors may also be treated with chemotherapy. Despite current treatment paradigms, however, penile SqCC is associated with significant morbidity. Recent advances in immunotherapy offer promising new therapeutic options for some solid tumors. For example, membranous expression of PD-L1, which facilitates immune checkpoint pathway activity, is present in a subset of primary SqCC of other organs, and immune checkpoint inhibitors (including anti-PD-L1 immunotherapy) are currently in clinical trials for treatment of these tumors. PD-L1 expression, however, has not been previously assessed in penile SqCC.

Design: Penile SqCC cases from 2005 to 2013 were retrospectively identified from the surgical pathology record database at a single large academic institution. Material from 37 patients, including 37 primary tumors and 9 matched inguinal lymph node metastases, was available for analysis, and immunohistochemistry (IHC) using an antiPD-L1 primary antibody (clone $5 \mathrm{H} 1$ ) was performed on representative whole tissue sections. PD-L1 IHC was assessed for the percentage of tumor cells with membranous staining by two study pathologists. Primary tumors were considered positive for PD-L1 expression if $\geq 5 \%$ of tumor cells showed membranous expression.

Results: 23 primary tumors were positive for PD-L1 expression (median \% tumor cells staining $=20$, range $=5-70$ ), and PD-L1 expression in primary tumors strongly correlated with matched lymph node metastases (Pearson correlation coefficient $=$ $0.793 ; \mathrm{p}<0.01$ ). While positive PD-L1 expression was not associated with pathologic T stage, primary tumors with regional lymph node metastases were significantly more likely to be positive for PD-L1 expression (pN1-3 vs. pN0/NX; $p=0.02$ ). Similarly, primary tumors with higher histologic grade (well vs. moderate vs. poor; $\mathrm{p}=0.07$ ) and conventional morphology (usual type vs. others; $p=0.04$ ) were more likely to be positive for PD-L1 expression.

Conclusions: Nearly two-thirds of penile SqCC are positive for PD-L1 expression, with high correlation between primary tumors and inguinal nodal metastases. Positive PD-L1 expression is more common in primary tumors with higher histologic grade, conventional (usual type) morphology, and regional lymph node metastasis. These data provide a rationale for targeted anti-PD-L1 immunotherapy as possible treatment for penile SqCC.
989 The Performance of a 17-Gene Genomic Prostate Score (GPS), PTEN Fluorescence In Situ Hybridization (FISH), and PTEN Immunohistochemistry (IHC) as Predictors of Clinical Recurrence (cR) in Prostate Cancer (PCa) Following Radical Prostatectomy (RP)

Cristina Magi-Galluzzi, Athanasios C Tsiatis, Michael J Bonham, Dejan Knezevic, Tara Maddala, Anne Dee, Sara Falzarano, H Jeffrey Lawrence, Phillip G Febbo, Eric A Klein. Cleveland Clinic, Cleveland, $\mathrm{OH}$; Genomic Health, Inc., Redwood City, CA. Background: PTEN loss by FISH or IHC has been reported in $20-60 \%$ of localized PCa and is viewed as an adverse prognostic factor. The GPS is an analytically and clinically validated RT-PCR assay that measures tumor aggressiveness in biopsies of men with early stage PCa. Gene selection for GPS was based on the strength of association between their expression and clinical outcomes. The prognostic value of GPS and PTEN loss in predicting $\mathrm{cR}$ after RP for clinically localized PCa was assessed.

Design: RPs from 441 patients (Prognostic Grade Group 1 - 23.7\%, 2 - 43.5\%, 3 $18.8 \%, 4-7.6 \%, 5-6.5 \%$ ) with a median follow up of 5.3 years were used to develop GPS. PTEN status was assessed by FISH and IHC in 287 and 369 of these patients, respectively, using tissue microarrays. Evaluable patients were representative of the full cohort. PTEN FISH loss was defined as hemizygous or homozygous deletion; PTEN IHC loss was defined as homogeneous loss of staining. GPS was generated using tumor RNA from microdissected RPs. Multivariable Cox models were used to analyze $\mathrm{cR}$ (defined as distant or local recurrence), and regression to the mean correction was used for GPS. Results: $38 \%$ of patients had PTEN loss by FISH and $25 \%$ by IHC. While PTEN status by FISH or IHC was associated with $\mathrm{cR}(\mathrm{p}<0.05)$ in univariate analysis, after adjusting for GPS, neither FISH nor IHC PTEN was a significant predictor for $c R(p>0.1)$. Concordance between the 2 PTEN methodologies was $66 \%(\mathrm{p}<0.001)$, whereas PTEN status and GPS were only weakly correlated (Spearman corr $=0.2-\mathrm{FISH}, 0.3-\mathrm{IHC}$ ) and a broad range of overlapping GPS was observed in each PTEN category. GPS remained strongly associated with $\mathrm{cR}$ after adjusting for PTEN $[\mathrm{HR} / 20$ units $=4.0(95 \%$ CI $2.1,7.7)$ by FISH; HR/20 units $=4.0(2.3,6.7)$ by IHC]. Of PTEN deleted cases (by FISH), $31 \%$ with the lowest GPS had $<2 \% 10$-year rate of cR. Of PTEN intact cases (by FISH), 29\% with the highest GPS had a 14\% 10-year rate of $\mathrm{cR}$.

Conclusions: GPS is a significant predictor of $\mathrm{cR}$ following RP after adjustment for PTEN status assessed by FISH or IHC. PTEN is not a significant predictor of $\mathrm{cR}$ after adjustment for GPS. GPS can identify a group of PTEN loss patients with a very favorable prognosis and a group of PTEN intact patients with unfavorable prognosis.

990 Stathmin1 is a Sensitive and Specific Biomarker for High Grade Urothelial Carcinomas

Douglas A Mata, Brooke E Howitt, Justine A Barletta, Michelle Hirsch. Brigham and Women's Hospital, Boston, MA.

Background: Stathmin 1 (STMN1), a microtubule destabilizing protein, regulates cytoskeletal dynamics, cell cycle progression, mitosis, and cell migration, and is influenced by $\mathrm{p} 53, \mathrm{p} 27$, and PI3K/Akt pathway activation. STMN1 expression has been shown to be a useful diagnostic biomarker in fallopian tube intra-epithelial carcinomas and squamous intraepithelial lesions of the cervix. It is unclear whether STMN1 is useful in the diagnosis of urothelial carcinoma. The goals of this study were to determine if STMN1 can be used to differentiate between 1) urothelial carcinoma in situ (CIS) and benign/reactive/denuded urothelium (BUM); 2) CIS and the 'shoulder' of a papillary urothelial carcinoma (PUC); and (3) high grade and low grade PUCs.

Design: 114 biopsies were examined, including 46 BUMs, 25 CISs, 24 LG PUCs, and 19 HG PUCs. All cases were examined by H\&E, but scored blindly for STMN1 (absent or scattered basal cells only $=$ negative vs near full to full thickness staining $=$ positive), p53 (wild type vs mutant), CK20 (umbrella or scattered cells = negative vs full thickness $=$ positive), and Ki67 (low vs elevated). Standard binary classification analysis was used to compute the sensitivity and specificity of the markers. Statistical significance was defined as a two-tailed $\mathrm{P}$ value $<0.05$.

Results: Immunohistochemical results are presented in Table 1, and sensitivity and specificity for single or combined biomarkers is presented in Table 2. Based on these results, both STMN1 and p53 appear to be far superior to CK20 for bladder biopsy evaluation.

\begin{tabular}{|l|l|l|l|l|}
\hline & $\begin{array}{l}\text { CK20 } \\
\% \text { positive }\end{array}$ & $\begin{array}{l}\text { p53 } \\
\% \text { mutant }\end{array}$ & $\begin{array}{l}\text { STMN1 } \\
\% \text { positive }\end{array}$ & $\begin{array}{l}\text { Ki67 } \\
\% \text { elevated }\end{array}$ \\
\hline BUM & 20 & 0 & 2 & 2 \\
\hline CIS & 40 & 64 & 88 & 80 \\
\hline HG PUC & 58 & 37 & 89 & 89 \\
\hline LG PUC & 46 & 4 & 4 & 4 \\
\hline
\end{tabular}

\begin{tabular}{|l|l|l|l|}
\hline & $\begin{array}{l}\text { CIS vs BUM } \\
\text { (sensitivity / specificity) }\end{array}$ & $\begin{array}{l}\text { CIS vs HG PUC } \\
\text { (sensitivity / specficity) }\end{array}$ & $\begin{array}{l}\text { HG PUC vs LG PUC } \\
\text { (sensitivity / specificity) }\end{array}$ \\
\hline $\mathrm{CK20}$ & $.53 / .71$ & $.48 / .35$ & $.50 / .62$ \\
\hline $\mathrm{p53}$ & $1.0 / .84$ & $.70 / .57$ & $.88 / .66$ \\
\hline $\mathrm{STMN1}$ & $.96 / .94$ & $.56 / .40$ & $.94 / .92$ \\
\hline $\mathrm{p53}+\mathrm{CK20}$ & $1.0 / .71$ & $.60 / .44$ & $1.0 / .65$ \\
\hline $\begin{array}{l}\mathrm{p53}+ \\
\text { STMN1 }\end{array}$ & $1.0 / .82$ & $.71 / .57$ & $1.0 / .62$ \\
\hline
\end{tabular}

Conclusions: Although STMN1 and p53 show similar sensitivity for CIS, STMN1 appears to be more specific for CIS and the distinction of LG vs HG PUC. Neither p53 nor STMN1 is very useful for distinguishing CIS and the shoulder of a papillary lesion. In addition to being a useful diagnostic biomarker, these results suggest that STMN1 overexpression in high grade urothelial carcinomas may potentiate aberrant cell proliferation, loss of polarity, and/or migration during early tumorigenesis. 
991 Lymphovascular Invasion of the Spermatic Cord in the Absence of Soft Tissue Invasion is a High Risk Finding in Testicular Nonseminomatous Germ Cell Tumors

Brandi C McCleskey, Julie Jorns, David Y Lu, Andres Matoso, Lauren E Schwartz, Jonathan I Epstein, Jennifer B Gordetsky. The University of Alabama at Birmingham, Birmingham, AL; University of Michigan, Ann Arbor, MI; David Geffen School of Medicine at UCLA, Los Angeles, CA; Brown University, Providence, RI; University of Pennsylvania, Philadelphia, PA; The Johns Hopkins Hospital, Baltimore, MD. Background: Current guidelines define testicular germ cell tumors (GCTs) with lymphovascular invasion (LVI) as stage pT2 and those with spermatic cord involvement as stage pT3. Lymphovascular invasion within the spermatic cord without direct involvement of the cord soft tissues is not currently addressed by the AJCC TNM system and its clinical significance has yet to be studied. We investigated the clinical significance of $\mathrm{LVI}$ in the spermatic cord in patients with testicular GCTs

Design: We performed a retrospective, multi-institutional review of testicular GCTs, 2000-2015. Cases with LVI of the spermatic cord without soft tissue invasion of the cord were selected. Clinico-pathologic features were reviewed.

Results: We identified 20 patients, mean age 33, diagnosed with testicular GCTs that had LVI in the spermatic cord without soft tissue invasion of the cord. Mean followup was 31 months. 16 cases were called pT2, one was called pT3, and 2 were not staged. 13/20 (65\%) patients had non-seminomatous germ cell tumors (NSGCT) and $7(35 \%)$ had seminomas. $12 / 13(92 \%)$ NSGCT were of mixed histology and one was pure embryonal. 10/13 (77\%) NSGCT had involvement of the rete testis, $2 / 13(15 \%)$ had involvement of the hilar fat, and 3/13 (23\%) had LVI present at the spermatic cord margin. 9/13 (69\%) patients with NSGCT presented with metastatic disease, 4/13 $(31 \%)$ had disease progression or recurrence after chemotherapy, and one patient died. $2 / 4(50 \%)$ patients who did not initially present with metastatic disease had disease recurrence. 7/20 (35\%) patients had seminomas. 5/7 (71\%) seminomas had rete testis invasion, 3/7 (43\%) had involvement of the hilar fat, and 2/7 (29\%) had LVI present at the spermatic cord margin. 1/7 (14\%) presented with metastatic disease and none had disease recurrence or died.

Conclusions: Patients with NSGCT and LVI in the spermatic cord, without soft tissue invasion of the cord, present at a higher clinical stage. Although this represents a rare finding, our results suggest it is a high risk feature with clinical outcomes like those of pT3 tumors. How to stage these tumors remains controversial.

\section{Submitting the Entire Packet Increases Lymph Node Yield in} Cystectomy Pelvic Lymphadenectomy Specimens

Rebekah McIntosh, Soroush Rais-Bahrami, Jennifer B Gordetsky. UAB, Birmingham, AL.

Background: The total number of lymph nodes removed during radical cystectomy has important prognostic and therapeutic benefits. Studies have shown that survival continues to improve with the more lymph nodes removed. The total number of lymph nodes removed also acts as a surrogate for the quality of the lymphadenectomy. Grossly identifying lymph nodes may be complicated by lymph node size and resident skill level. We investigated the benefit of introducing a grossing method requiring the entire lymph node packet to be submitted.

Design: We retrospectively examined 56 radical cystectomy and cystoprostatectomy cases with concurrent pelvic lymph node dissections at our institution done between 2012 and 2015. A new method requiring total submission of lymph node packets was introduced in cases beginning in 2014. We assessed the difference in lymph node yield, number of lymph node packets submitted, number of blocks, and the proficiency of finding lymph nodes based on PGY level.

Results: 27 cases using the new lymph node grossing method of total packet submission and 29 cases utilizing the older method of individual lymph node submission were identified. Age, pathologic T and N stage, and PGY-level grossing each case were not significantly different between the groups. Significantly more lymph nodes were found when the entire lymph node packets were submitted (mean; range): 25.2; (566) vs $15.9 ;(1-46) \mathrm{p}=0.011$. The new technique required significantly more blocks for processing 19.0 vs $12.4(p=0.017)$. A significant decrease was seen in the number of lymph node packets submitted by the surgeon since implementation of the new method: $2.2 ;(1-6)$ vs $3.9 ;(1-7), \mathrm{p}<0.001$. The PGY level of resident grossing was not significantly associated with number of nodes found or the benefit of increased nodes with implementation of the new technique.

Conclusions: At our institution, surgeons have decreased the average number of lymph node packets submitted with radical cystectomy cases. Even with this decrease in specimens, the introduction of entire lymph node packet submission significantly increases the total number of lymph nodes identified, independent of PGY level grossing the case.
993 Validation of Reactive Stroma as an Adverse Prognostic Factor for Gleason Grade 3+3=6 and 3+4=7 Prostatic Adenocarcinomas (PCA) In the Canary Retrospective Radical Prostatectomy (RP) Cohort

Jesse K McKenney, Wei Wei, Sarah J Hawley, Heidi Auman, Lisa Newcomb, Hilary Boyer, Ladan Fazli, Jeff Simko, Antonio Hurtado-Coll, Dean Troyer, Maria STretiakova, Funda Vakar-Lopez, Peter R Carroll, Matthew R Cooperberg, Martin E Gleave, Raymond Lance, Daniel Lin, Peter S Nelson, Ian Thompson, Lawrence True, Ziding Feng, James D Brooks. Cleveland Clinic, Cleveland, OH; UT MD Anderson, Houston, TX; Canary Foundation, Palo Alto, CA; Fred Hutchinson CRC, Seattle, WA; University British Columbia, Vancouver, BC, Canada; UCSF, San Francisco, CA; Eastern Virginia MS, Norfolk, VA; UW Medical Center, Seattle, WA; UT San Antonio, San Antonio, TX; Stanford University, Stanford, CA.

Background: Predicting the clinical course of a PCA in an individual patient is critical to active surveillance (AS). Our aim was to optimize grading for AS.

Design: We developed a histologic method of recording individual patterns of PCA independent from standard Gleason grade and recorded all patterns present (regardless of extent) from patients in the Canary RP TMA cohort, where highest grade PCA was studied (Adv Anat Pathol 2013;20:39). All were also Gleason graded utilizing current ISUP criteria. Recurrence free survival (RFS) was defined as time until: 1) single serum PSA level $>0.2 \mathrm{ng} / \mathrm{mL}>8 \mathrm{wk}$ after RP; and/or 2) receipt of salvage or secondary therapy after RP; and/or 3) clinical/radiologic evidence of metastasis after RP; and/or 4) PCA death. We correlated histologic patterns with RFS to compare their relative prognostic strength using univariate and multivariate Cox proportional hazard analysis in the 1275 patients with TMA cores showing PCA (significance at $\mathrm{p} \leq 0.05$ ).

Results: We analyzed the Gleason score $3+3=6(n=522)$ and $3+4=7(n=332)$ categories. In univariate analyses for Gleason $3+3=6$ carcinomas, stromogenic patterns and patterns borderline for $3+4=7$ (i.e. focal poorly formed glands versus tangentially sectioned glands) were associated with worse RFS $[p=0.05$ and 0.001 , respectively]. In the grade $3+4=7$ category, the two stromogenic patterns were significantly associated with worse $\mathrm{RFS}[\mathrm{p}=0.008$ and 0.006$]$. Cribriform growth was associated with worse RFS compared to poorly formed glands. All patterns with mucin rupture graded as $3+4=7$ were associated with improved RFS, and two patterns were significant $[\mathrm{p}=0.03$ and 0.04$]$. The multivariate analysis for these 2 Gleason score groups validated that stromogenic patterns were significantly associated with worse RFS, adjusted for extraprostatic invasion, seminal vesicle invasion, margin status, age, and pre-operative PSA ( $p=0.01)$. Conclusions: These data suggest areas for optimizing Gleason pattern assignment and strongly support incorporating stromogenic patterns into the grading system for PCA.

994 Correlation of Gleason Score 7 in Prostate Adenocarcinoma on Needle Biopsy between General and Urological Pathologists: Influence of Percent Pattern $\mathbf{4}$ and Other Histological Factors

Abdelrazak Meliti, Francesca Khani, Mairo L Diolombi, Evita T Sadimin, Jonathan I Epstein. Johns Hopkins Hospital, Baltimore, MD.

Background: Recognizing Gleason pattern 4 in prostatic needle biopsies is crucial and can impact clinical management and treatment options for patients diagnosed with prostatic adenocarcinoma. Recently, it has been recommended to record percent pattern 4 when Gleason score 7 cancer is the highest grade in a case.

Design: 405 prostate needle core biopsies received from other institutions for a second opinion at our institution from February-June 2015 were prospectively diagnosed with prostatic adenocarcinoma Gleason score 7 on review by a consultant genitourinary pathologist. Percentage of core involvement, percentage of Gleason pattern 4 per core, distribution of Gleason pattern 4 (clustered, scattered), morphology of pattern 4 (cribriform, non-cribriform), and whether the cancer was continuous or discontinuous were recorded.

Results: 290 biopsies (71.6\%) were assigned Gleason $3+4$ and 115 biopsies (28.4\%) were assigned Gleason $4+3$ on the consultant's review. When the consultant assigned $3+4$, there was agreement in with the referring pathologists in 187 biopsies $(64.5 \%)$; 79 biopsies $(27.2 \%)$ were under-graded and 24 biopsies $(8.3 \%)$ were over-graded relative to the consultant. When the consultant assigned $4+3$, there was agreement with the referring pathologists in 54 biopsies (47\%); 50 biopsies (43.5\%) were undergraded and 11 biopsies $(9.5 \%)$ were over-graded relative to the consultant. Greater agreement was noted between the consultant and referring pathologists when pattern 4 was clustered in biopsies with Gleason $(3+4)$ and $(4+3)(\mathrm{P}=0.009)$. The percentage of core involvement, morphology of pattern 4 , and continuity of cancer did not affect the agreement between the consultant and referring pathologists. There was a trend $(p=0.06)$ for better agreement based on the percent of pattern 4 . When the consultant diagnosed Gleason $3+4$ and the contributors graded lower, in $80 \%$ of the cases there was $\leq 20 \%$ pattern 4 . When the consultant diagnosed Gleason $4+3$ and the contributors graded lower, $70 \%$ of the cases had $50 \%-70 \%$ pattern 4 .

Conclusions: Including percent pattern 4 in reports on prostate adenocarcinoma can help explain discrepant grading between pathologists in borderline cases. This study also calls in to attention other contributing factors for grading differences between observers based on distribution of the pattern 4 .

995 Histopathological Risk Scoring System as a Tool for Predicting Lymph Nodal Metastasis in Penile Squamous Cell Carcinoma

Santosh Menon, Aakash Sali, Gagan Prakash, Ganesh Bakshi, Amit Joshi, Vedang Murthy, Umesh M Mahantshetty, Sangeeta Desai. Tata Memorial Centre, Mumbai, India. Background: Penile cancer is an aggressive neoplasm and nodal metastasis is the most important prognostic factor which determines the outcome. Elective prophylactic lymphadenectomy may cause severe morbidity. There is a paucity of tools predicting nodal metastasis in penile cancer. 
Design: Retrospective clinicopathologic analysis of consecutive penile squamous cell carcinoma patients who had undergone primary surgical treatment (penectomy specimens and circumcisions) with either upfront or follow-up unilateral/bilateral inguinal lymph node dissections from 2007-2012 was undertaken. Histopathology slides were reviewed and a scoring system was devised by adding up the grade of tumor (score 1-3), anatomical level of invasion (score 1-3) and pattern of infiltration (score 1-3). The scores were correlated with nodal metastasis, disease free survival and overall survival. Multivariate analysis was performed using logistic regression models. Survival curves were calculated using the Kaplan-Meier method and Cox regression method. Comparison between curves were done by Mantel-Cox (log-rank) test.

Results: A total of 162 cases of primary resections of penis with unilateral or bilateral groin node dissection (GND) were identified during the study period.62/68 patients $(91.17 \%)$ and $58 / 94$ patients $(61.7 \%)$ had nodal metastasis on upfront and follow-up nodal basin surgeries, respectively. The risk groups created are shown in table 1.

\begin{tabular}{|l|l|l|}
\hline Risk scoring groups (Modified prognostic index) & Nodal involvement & p value \\
\hline Low risk (score 3 and 4) & $14.3 \%$ & \multirow{3}{*}{ p $<0.001$} \\
\cline { 1 - 2 } Intermediate risk (score=5) & $52.6 \%$ & \\
\hline High risk (score 6 to 9) & $83.7 \%$ & \\
\hline
\end{tabular}

Follow-up was available in 145 patients (89.5\%). Median follow- up was 21 months (1-96 months). Median disease free survival (DFS) was 8 months. Median overall survival (OS) for the whole group was 23 months. Median OS with and without nodal metastasis was 18 months and 30 months respectively. The histological scoring system $(\mathrm{p}=0.045)$ and risk groups $(\mathrm{p}=0.005)$ had statistically significant correlation with DFS but not with OS.

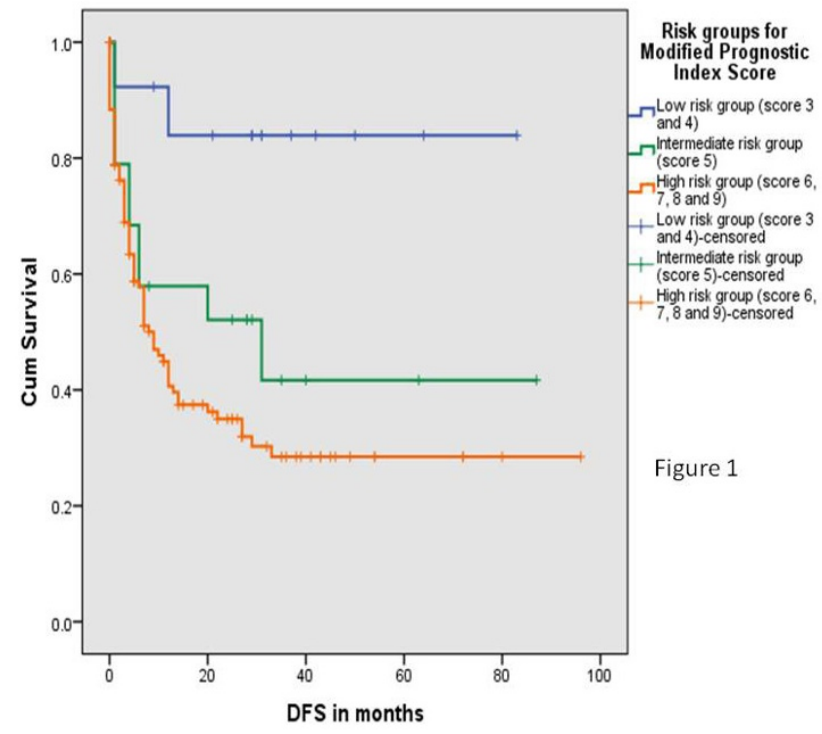

Conclusions: Histopathological risk stratification can predict nodal metastasis and aid in planning management of penile cancer patients.

\section{Uroplakin 2: A Specific Marker of Urothelial Carcinoma}

Ioana Moisini, Rajiv Dhir, Aatur Singhi, Aditi Ranade, Esther Elishaev, David Dabbs, Rohit Bhargava. Magee-Womens Hospital, Pittsburgh, PA; UPMC, Pittsburgh, PA; UHS Wilson Medical Center, Johnson City, NY.

Background: Uroplakin (UPs) are a group of transmembrane glycoproteins localized to the asymmetric unit membrane of the urothelium. Currently UPIII is commonly used as urothelium specific antibody but its sensitivity is less than optimal. We compared the sensitivity of another similar protein UPII with UPIII in 55 bladder urothelial carcinomas and in 19 fetal bladder tissues. We also analyzed UPII reactivity in 439 carcinomas from various sites for its specificity.

Design: Bladder urothelial carcinoma tissue microarray (TMA) and fetal bladder tissues were stained for both UPII and UPIII. All other carcinomas were stained for UPII which were represented on TMAs except for gynecologic squamous cell carcinomas. The tissue reactivity was semi-quantitatively scored using the $\mathrm{H}$-score method where the score ranges from 0 (no reactivity) to 300 (strong reactivity). An H-score of $>10$ was considered positive.

Results: All 19 fetal bladder tissues were strongly positive for both UPII and UPIII. No staining was identified for UPII in normal squamous epithelium in 13 cases tested. UPII versus UPIII reactivity in bladder urothelial carcinoma is reported in table 1

\begin{tabular}{|l|l|l|l|}
\hline Table 1 & UPII neg & UPII pos & Total \\
\hline UPIII neg & 31 & 17 & 48 \\
\hline UPIII pos & 1 & 6 & 7 \\
\hline Total & 32 & 23 & 55 \\
\hline
\end{tabular}

UPII positive in $42 \%$ (23/55); UPIII positive in $13 \%$ (7/55). Average $\mathrm{H}$-score (in positive cases only) was slightly higher in UPII+ cases compared to UPIII ( 99.4 versus $50.8 ; \mathrm{p}=0.1255)$. If all cases are taken into consideration, then UPII $\mathrm{H}$-score was significantly higher than UPIII $(\mathrm{p}=0.0005)$.
UPII reactivity in non-bladder tumors is reported in table 2 .

\begin{tabular}{|l|l|}
\hline Table 2 & UPII + /Total cases \\
\hline Invasive squamous CA & $0 / 11$ \\
\hline Squamous cell CA in-situ & $0 / 3$ \\
\hline Endocervical adenoCA & $0 / 32$ \\
\hline Endometrial adenoCA & $0 / 51$ \\
\hline Ovarian adenoCA & $0 / 52$ \\
\hline Pancreatic adenoCA & $1 / 126^{*}$ \\
\hline CholangioCA & $0 / 57^{*}$ \\
\hline Breast adenoCA (female) & $0 / 53$ \\
\hline Breast adenoCA (male) & $0 / 54$ \\
\hline $\begin{array}{l}\text { *Positive case with H-score of } 15 . \text { Two additional pancreatic and one cholangio case with } \\
\text { H-score 1-10 (considered negative). }\end{array}$ \\
\hline
\end{tabular}

Conclusions: UPII is more sensitive than UPIII for urothelial carcinomas and is relatively specific. UPII stains only rare non-urothelial tumors and the reactivity is often weak. Lack of UPII reactivity in breast carcinomas is helpful as both urothelial and breast cancers share expression of GATA-3. UPII may also be used to distinguish urothelial from gynecologic squamous cell carcinomas, with positive staining favoring urothelial origin.

997 Lymph Nodes Metastasis in Patients with Renal Cell Carcinoma: Correlation with Histologic Subtype and Other Pathologic Features Gabriel E Morey, Jae Y Ro, Luan D Truong, Alberto G Ayala, Steven S Shen. Houston Methodist Hospital and Weill Medical College of Cornell University, Houston, TX. Background: Approximately $25 \%$ of patients with renal cell carcinoma (RCC) have distant metastasis at the time of diagnosis. However, regional lymph node metastasis is less common and the role of lymphadenectomy at the time of nephrectomy is not well defined and controversial. The aim of this study is to determine whether pathologic features of tumor can predict nodal metastasis.

Design: To identify any histologic features that are associated with nodal metastasis, we reviewed 465 radical nephrectomies (1990-2015) with lymph node dissection and correlated the histologic subtype, tumor size, Fuhrman nuclear grade, and presence of sarcomatoid changes with nodal metastasis. Fisher's exact or c2 test was used for comparing categorical variables between the groups.

Results: Of the 465 patients with nephrecomy and nodal dissection, lymph nodal metastases were identified in $78(16.8 \%)$ patients. Node metastases were found in $13.2 \%(48 / 364)$ of clear cell RCC, $29.0 \%(18 / 62)$ of papillary RCC, $0 \%(0 / 21)$ of chromophobe RCC, $100 \%$ (4/4) of collecting duct carcinoma (CDC), and 53.9\% (7/13) of unclassified RCC. Papillary RCC, CDC and unclassified RCCs were more likely to have nodal metastasis than clear cell and chromophobe RCCs $(\mathrm{P}<0.05)$. Sarcomatoid changes were seen in $33.3 \%$ (26/78) of nodal positive RCCs, but only in 5.4\% (21/ $387)$ of node-negative RCCs $(\mathrm{P}<0.001)$. The average tumor sizes for node-positive and node-negative groups were $10.1 \pm 3.4$ and $7.5 \pm 4.2$, respectively $(\mathrm{P}<0.001)$. High grade (Furhman's nuclear grade $3 \& 4$ ) tumors were seen in $88.4 \%(69 / 78)$ of node-positive RCCs, but only in $50.1 \%(194 / 387)$ of node-negative RCCs ( $<<0.001)$. Similarly, locally advanced tumors (T3 and T4) were seen much more often in node-positive group than node-negative group $(78.2 \%$ vs. $40.1 \%, \mathrm{P}<0.001)$.

\begin{tabular}{|l|l|l|l|}
\hline Histologic Subtype & Total Cases (n) & $\begin{array}{l}\text { Cases with Nodal } \\
\text { Metastasis (n) }\end{array}$ & $\begin{array}{l}\text { Nodal Metastasis } \\
(\%)\end{array}$ \\
\hline Clear cell RCC & 364 & 48 & 13.2 \\
\hline Papillary RCC & 62 & 18 & 29.0 \\
\hline Chromophobe RCC & 21 & 0 & 0 \\
\hline Unclassified RCC & 13 & 7 & 53.8 \\
\hline Xp11 translocation RCC & 1 & 1 & 100 \\
\hline
\end{tabular}

Conclusions: Certain histological subtypes (i.e. papillary RCC, CDC, and unclassified), greater tumor size, high nuclear grade and stage, and sarcomatoid change are significantly associated with nodal metastasis. This information may have implications for surgical planning for lymph node dissection at the time of radical nephrectomy.

998 Expression of miRNAs 200c and 33a That Negatively Control HAS2 and HAS3 in Localized Prostate Cancer

Caio M Moura, Sabrina T Reis, Jose Pontes, Nayara Viana, Nelson Dip, Miguel Srougi, Katia RM Leite. University of Sao Paulo Medical School, Sao Paulo, Brazil.

Background: Micro RNAs (miRNAs) are small noncoding molecules with important role in the control of gene expression. miRNA 200c and 33a have as target the hyaluronan synthases HAS2 and HAS3, which promote synthesis of hyaluronic acid (HA). HA is a major component of the extracellular matrix related to tissue repair, neovascularization, epithelial mesenchymal transition, tumor progression and development of metastasis. Our aim is to study the expression of miRNAs 200c and 33a and their targets HAS2 and HAS3 in localized prostate cancer (PCa).

Design: 49 surgical specimens from patients diagnosed with $\mathrm{PCa}$ who underwent radical prostatectomy were the subject of the study. The synthesis of cDNA was made from $0,5 \mu \mathrm{g}$ total RNA and the expression levels of the genes and miRNAs were detected using quantitative real time polymerase chain reaction (qRT-PCR) and the formula $2^{-\triangle \Delta C T}$. The control group consisted of 6 patients with benign prostatic hyperplasia (BPH). Results: The HAS2 and HAS3 were overexpressed in $92 \%$ and $94 \%$ of PCa respectively. The median and range were $4.46(0.41-83.4)$ and $3.56(0.62-343.22)$. MiRNAs 200c and $33 \mathrm{a}$ were underexpressed in $77.5 \%$ and $75.5 \%$ of PCa cases. When we correlate 
the expression of miRNAs with key prognostic factors, we found that lower expression of miR-200c was related to higher Gleason score $(\mathrm{p}=0.031)$ and patients with higher PSA levels $\geq 10 \mathrm{ng} / \mathrm{ml}$ had lower expression of $33 \mathrm{a}(\mathrm{p}=0.026)$.

Conclusions: We showed that HAS2 and HAS3 are overexpressed and miRNAs 200c and 33a, that controls negatively the expression of HAS2 and HAS3 are underexpressed in localized $\mathrm{PCa}$ and are both related to important and unfavorable prognostic factors. With this result, we suggest that miRNAs 200c and 33a are potential prognostic markers for PCa.

999 Prognostic Significance of Lymphatic, Vascular and Perineural Invasion in Bladder Cancer Treated by Radical Cystectomy

Prasuna Muppa, Sounak Gupta, Igor Frank, Stephen A Boorjian, R Jeffrey Karnes, $R$ Houston Thompson, Prabin Thapa, Robert F Tarrell, Loren P Herrera Hernandez, Rafael E Jimenez, John C Cheville. Mayo Clinic, Rochester, MN.

Background: The prognostic significance of lymphatic invasion (LI), vascular invasion (VI) and perineural invasion (PNI) in bladder cancer (BC) patients uniformly treated with radical cystectomy $(\mathrm{RC})$ are not well characterized. Prior studies using smaller cohorts have generated conflicting results regarding the role of LI, VI and PNI in predicting cancer -specific survival (CSS), and local recurrence specific survival (LRSS). Design: We studied a retrospective cohort of $1504 \mathrm{BC}$ patients (including 964 cases of conventional urothelial carcinoma [UC] and 540 cases of variant subtypes) uniformly treated with RC from our institutional registry. The mean follow up period was 10.6 years. All cases were re-reviewed by a urologic pathologist for bladder cancer subtype, stage, LI, PNI, and VI. LI, VI and PNI were defined by the presence of tumor cells within endothelial-lined lymphatic space, tumor cells within smooth muscle-lined blood vessels, and by tumor invasion into the perineural sheath, respectively. These results were correlated with relevant perioperative and clinical variables including outcome. Results: Of the 1504 patients, 965 (64\%) cases with no LI, VI and PNI were considered the reference group. $306(20 \%)$ patients had LI only, $59(4 \%)$ had VI only, $54(4 \%)$ had PNI only, and $120(8 \%)$ had a combination of LI, VI, and PNI. Multivariate analysis showed that, after adjusting for clinical and perioperative variables, LI and VI were significantly associated with CSS ( $\mathrm{HR}=1.7, p<0.0001$ and $\mathrm{HR}=1.5, \mathrm{p}=0.02$, respectively) when compared to the reference group. The frequency of LI, VI and PNI is varied by bladder cancer subtype.

Conclusions: LI is strongly associated with outcome in patients undergoing $\mathrm{RC}$ for $\mathrm{BC}$, and VI to a lesser extent. These features should be clearly defined and reported separately. The frequency of LI, VI and PNI vary by bladder cancer subtype.

\section{Clonality of Urothelial Carcinoma Developing in the Bladder} Following Radical Nephroureterectomy

Paari Murugan, Eugene K Cha, Ying-Bei Chen, Sahussapont J Sirintrapun, Anuradha Gopalan, Samson W Fine, Satish K Tickoo, Victor E Reuter, John P Sfakianos, Sasinya N Scott, Gopa Iyer, Aditya Bagrodia, Ronak Shah, Dean F Bajorin, Jonathan E Rosenberg, Michael Berger, Bernard H Bochner, Jonathan A Coleman, David B Solit, Hikmat A Al-Ahmadie. University of Minnesota, Minneapolis, MN; Memorial Sloan Kettering Cancer Center, New York, NY.

Background: Urothelial carcinoma can often be multifocal and may involve both the bladder and upper tract synchronously or metachronously. To explain this phenomenon, two hypotheses have been proposed: the 'field effect' hypothesis - urothelial cells are primed to undergo malignant transformation by exposure to carcinogens, and the clonal, or 'single progenitor cell,' hypothesis - tumors arise from intraluminal seeding of transformed cells.

Design: To examine their clonal relationships, we compared the genomic profiles of primary upper tract urothelial carcinoma (UTUC) tumors and metachronous bladder tumors (intravesical recurrences) in patients treated with radical nephroureterectomy (RNU) and subsequent transurethral resection. Specimens were analyzed using a nextgeneration, targeted sequencing assay designed to identify point mutations, indels, and copy number alterations in 341 cancer-associated genes.

Results: We analyzed 16 primary UTUC tumors and 41 intravesical recurrences in patients treated with RNU. The median number of intravesical recurrences per patient was 2 (range 1-7) and the interval from RNU to intravesical recurrence ranged from 3.5 months to 129 months. With an average sequencing coverage of 516x, there was strong evidence to support the clonal relationship between primary UTUC tumors and subsequent bladder tumors. In all subsequent bladder tumors, the majority of somatic mutations present in the primary UTUC tumors (median=7, range 4-39) were detected $(128 / 146,88 \%)$. In one example, a patient followed with periodic cystoscopy/cytology who had been disease free for 5.5 years then developed 7 bladder tumors over the next 44 months, each with the identical mutation profile ( 8 mutations) as the primary tumor. Conclusions: In this cohort, bladder tumors following RNU represent true intravesical recurrences, with almost all tumors sharing the same somatic mutation profile as the primary UTUC tumor. These findings argue against the concept of field effect as a mechanism for tumor recurrence and multifocality in urothelial carcinoma and may have important implications for surgical techniques to minimize the risk of intraluminal seeding and the delivery of intravesical therapy following RNU.
1001 Aurora A in Metastatic Castration Resistant Prostate Cancer (mCRPC)

Daniel Nava Rodrigues, Niven Mehra, Ines Figueiredo, Mateus Crespo, Ruth Riisnaes, Susana AA Miranda, Ana Ferreira, Nina Tunariu, Raquel Perez-Lopez, Gunther Boysen, Adam Sharp, Diletta Bianchini, Michael Kolinsky, Pasquale Rescigno, Zafeiris Zafeiriou, Joaquin Mateo, Johann S De Bono. ICR, London, United Kingdom; Royal Marsden Hospital, London, United Kingdom.

Background: Amplification and overexpression of Aurora kinase A (AURA ${ }^{\mathrm{AMP}}$ and AURA $^{\mathrm{OE}}$ ) occurs in diverse solid tumor types, associates with a higher proliferative index and poorer outcome. It may select for tumours susceptible to AURA inhibition. Here we analyse AURA ${ }^{\mathrm{AMP} / \mathrm{OE}}$ and neuroendocrine differentiation (NED) in metastatic castration resistant prostate cancer (mCRPC).

Design: Metastatic CRPC biopsies were analyzed for AURA by FISH; amplification was defined as $\geq 4$ signals and a ratio $\geq 2$. Tumours were further characterized by immunohistochemistry for AURA, Ki67, androgen receptor(AR; amino-terminal epitope) staining (ARPOS vs ARNEG), and neuroendocrine markers chromogranin-A, synaptophysin and CD56. AURA and Ki67 were scored as \% of positive cells and defined as overexpressed or high when scored above the cohort's median. 'Neuroendocrine differentiation (NED)' was defined as $\geq 1$ positive NEmarker in $\geq 30 \%$ of tumor cells. The covariates tested were AURAOE and High Ki67 (both dichotomized at median), AURAAMP, neutrophil-to-lymphocyte ratio (NLR), and established clinical prognostic variables including haemoglobin, lactate dehydrogenase (LDH), alkaline phosphatase, PSA and presence of visceral metastases.

Results: Fifty-six mCRPC biopsies were evaluated; biopsy sites included bone (35.7\%), nodes $(35.7 \%)$ and other $(28.6 \%)$. The median AURA and Ki67 scores were 10 (range $0-70$ ) and 50 (5-95), respectively. FISH data were obtained from 45 patients; AURA AMP was identified in 3 cases (6.67\%). Overall, 17 (30.4\%) tumors had NED biomarker positivity; 5 of these were AR ${ }^{\mathrm{NEG}}$. There was no association between NED biomarker positivity and $\mathrm{AURA}^{\mathrm{OE}}$ or AURA ${ }^{\mathrm{AMP}}$. AURA ${ }^{\mathrm{OE}}$ correlated with Ki67 expression $(\mathrm{r}=0.56$ with $\mathrm{P}<0.001)$. AURA ${ }^{\text {OE }}$ positivity associated with shorter overall survival (OS) from date of biopsy ( 10 vs $13 \mathrm{mo}, \mathrm{P}=0.04$ ); high Ki67 ( 9 vs $19 \mathrm{mo}, \mathrm{P}=0.016$ ), and NED ( 9 vs 13 mo, $\mathrm{P}=0.031$ ) were also prognostic. In univariate analysis, AURA ${ }^{\mathrm{OE}}$ and Ki67 associated with decreased OS, but AURA ${ }^{\mathrm{AMP}}$ did not. Other significant variables tested in a multivariate analysis(MVA) model were AR, NED, visceral mets, NLR and LDH. In MVA there was a relation between $\mathrm{AURA}^{\mathrm{OE}}$ (HR 5.36, with 95\% CI 1.83-15.7, $\mathrm{P}=0.002$ ) and poor OS, independent of NED status.

Conclusions: High AURA expression associates with poor prognosis in $\mathrm{mCRPC}$ and higher proliferation. AURA ${ }^{\text {OEAMPP }}$ did not associate with NED biomarkers.

\section{Monitoring CHD1 During Prostate Cancer Progression}

Daniel Nava Rodrigues, Gunther Boysen, Joaquin Mateo, Rossitza Christova, Ruth Riisnaes, Mateus Crespo, Theresa Y MacDonald, Susana AA Miranda, Ines Figueiredo, Veronica Gil, Sara Aziz, Niven Mehra, Adam Sharp, Pasquale Rescigno, Juan Miguel Mosquera, Christopher E Barbieri, Mark Rubin, Johann S De Bono. ICR, London, United Kingdom; Weil Medical College of Cornell University, New York, NY.

Background: Deletions in the gene Chromodomain Helicase DNA Binding Protein 1 (CHDI) are among the most frequent genomic alterations in prostate cancer (up to $20 \%$ ). CHDI is a ATPase-dependent helicase mediating a variety of biological processes by organizing chromatin structure. In hormone naive prostate cancer, loss of CHD1 has been associated with increased genomic instability and aggressiveness.

Design: We retrospectively identified 52 patients of whom HSPC and CRPC tissue was available for immunohistochemical analysis of CHD1, ERG, PTEN, AR and Ki67 as well as for CHD1 fluorescence in situ hybridization. To determine CHD1 gene status in corresponding CRPC plasma DNA we developed a digital droplet PCR assay. Relationship with outcome was analyzed using univariate Cox regression and log-rank analyses.

Results: By using Fluorescence In Situ Hybridization (FISH) and immunohistochemistry (IHC) in a cohort of $\mathbf{5 2}$ men who developed CRPC, we show that the frequency of loss of CHD1 does not change during disease progression (7.7\% in HSPC vs 9.6\% in CRPC). Homozygously deleted tumors demonstrate a tendency towards poorer prognosis but this observation lacks statistical power in our cohort. Interestingly, in patients that show CHD1 protein expression in their hormone naïve sample, a decrease of expression in the matched CRPC sample correlates with poorer overall survival $(\mathrm{OS})(\mathrm{p}=0.03)$. Although preclinical data suggest a role for CHD1 in androgen receptor (AR) activity (Burkhardt et al.), CHD1 expression did not correlate with AR protein level. Additionally, Ki67 expression did not show correlation with CHD1 levels of expression. Further molecular stratification confirms a known mutual exclusive relationship with the expression of the ERG transcription factor $(\mathrm{p}=0.048)$. Clinically, although decreased CHD1 protein expression during disease progression correlated with worse outcome (overall survival $(\mathrm{p}=0.03)$ and survival from diagnosis of CRPC $(\mathrm{p}=0.02))$, no significant impact on survival after docetaxel or abiraterone treatment was measured.

Conclusions: Here we monitor CHD1 deletion during prostate cancer progression. Our results show that this genomic marker defines a distinct molecular subgroup of prostate cancer. In addition, loss of CHD1 protein expression during disease progression is associated with poorer clinical outcome. 
1003 Natural History of Human Papillomavirus Infection in NonVaccinated Males: Low Clearance Probability in High-Risk Genotypes

Gabriella Nesi, Tommaso Cai, Sandra Mazzoli, Raffaella Santi. University of Florence, Florence, Italy; Santa Chiara Regional Hospital, Trento, Italy; Santa Maria Annunziata Hospital, Florence, Italy.

Background: Human papillomavirus (HPV) infection is one of the most common sexually transmitted infection in both genders. HPV is the main cause of cervical carcinomas in women, and is responsible for anal, penile and oropharyngeal cancer in men. It is therefore imperative to lower the prevalence of HPV infection in the male population. In this study, we aimed to investigate the clearance of type-specific genital HPV infection in a series of heterosexual, non-HPV vaccinated males whose female partners were positive to DNA-HPV tests.

Design: Men attending the same Sexually Transmitted Diseases Center between January 2005 and December 2006 were considered for this long-term longitudinal cohort study. All subjects $(\mathrm{n}=1009)$ underwent a urologic visit and microbiological tests on first void, midstream urine and total ejaculate samples. One hundred and five patients were positive to HPV-DNA ( $\mathrm{n}=105 ; 10.4 \%$; mean age: $34.8 \pm 5.8$ ) and consented to clinical examination and molecular diagnostic assays for HPV detection scheduled every six months (median surveillance period of 53.2 months; range: $48-71$ months). According to Munoz et al., HPV genotypes were classified into high-risk, probable high-risk and low-risk. HPV positive samples which did not hybridize with any of the type-specific probes were referred to as positive non genotype-able.

Results: At enrollment, the distribution of HPV genotypes was as follows: high-risk HPV ( $\mathrm{n}=37)$, probable high-risk HPV $(\mathrm{n}=6)$, low-risk HPV $(\mathrm{n}=23)$, non genotype-able HPV $(n=39)$. A high HPV genotype concordance between stable sexual partnersemerged (kappa $=0.92 ; \mathrm{p}<0.001)$. At the end of the follow-up period, 71/105 (67.6\%) subjects were negative for HPV (mean viral clearance time: 24.3 months). With regard to HPV genotype, viral clearance was documented in $14 / 37(37.8 \%)$ high-risk HPV cases, 6/6 (100\%) probable high-risk HPV cases, 20/23 (86.9\%) low-risk HPV cases, 31/39 (79.5\%) non genotype-able cases. The high-risk HPV genotypes showed the lowest rate and probability of viral clearance $(\mathrm{p}<0.001)$.

Conclusions: In our series, high-risk HPV infections were more likely to persist over time when compared with other HPV genotypes. The good HPV genotype concordance between female partners and enrolled males may be the key to extending vaccination programs to all men with high-risk HPV positive partners.

\section{TERT Promoter Mutations in Aggressive Variants of Urothelial} Carcinoma

Doreen Nguyen, Simeon Springer, Morgan Cowan, Diana Taheri, Gunes Guner, Maria Angelica Mendoza Rodriguez, Stephania Bezerra, Isabela W Cunha, Dilek Baydar, Maria DC Rodriguez, Yuxuan Wang, Christopher J VandenBussche, Kenneth Kinzler, Nickolas Papadopoulos, Bert Vogelstein, George J Netto. Johns Hopkins Hospital, Baltimore, MD; Sidney Kimmel Comprehensive Cancer Center, Baltimore, MD; Isfahan University of Medical Sciences, Isfahan Kidney Diseases Research Center, Isfahan, Islamic Republic of Iran; AC Camargo Cancer Center, Sao Paulo, Brazil; Hacettepe University, Ankara, Turkey.

Background: Somatic activating mutations in the promoter of the telomerase reverse transcriptase (TERT) gene are the most common genetic mutations in urothelial carcinoma (UC) of the bladder and upper urinary tract. Little is known, however, about $T E R T$-mutation status in the relatively uncommon but clinically aggressive plasmacytoid (PUC) and micropapillary (MPC) variants. Our group is persuing a TERT promoter mutations (TERT-mut) urine based screening assay for bladder cancer. We evaluated the presence of TERT-mut in PUC and MPC of bladder.

Design: We retrospectively identified $48 \mathrm{MPC}$ and 16 PUC cases from our archives (2005-2014). All slides were reviewed to confirm the diagnosis. 33 MPC cases and 10 PUC cases had FFPE blocks available for DNA analysis. Intratumoral areas of nonmicropapillary and non-plasmacytoid histology were also evaluated when present. Samples were analyzed with Safe-SeqS, a sequencing error-reduction technology, and sequenced using Illumina MiSeq as previously described (Kinde, I et al. Cancer Res 2013: 367)

Results: Overall, TERT-mut was detected in 91\% (39 of 43) of all cases. All 33 cases of pure MPC as well as UC with focal micropapillary features demonstrated TERT-mut. $56 \%$ of pure PUC cases were also positive. Similar to conventional UC, the predominant mutations identified occurred at positions - 124 (C228T) $(90 \%)$ and -146 (C250T) $(10 \%)$ bp upstream of the TERT ATG start site.

In heterogeneous tumors with focal variant histology,intratumoral concordant mutations were found in variant (MPC or PUC) and corresponsing conventional UC.

\begin{tabular}{|l|l|l|l|l|}
\hline Tumor Histology & $\begin{array}{l}\text { TERT-mut Positive } \\
\mathbf{n}(\%)\end{array}$ & $\begin{array}{l}\text { C228T } \\
\mathbf{n}(\%)\end{array}$ & $\begin{array}{l}\text { C250T } \\
\mathbf{n}(\%)\end{array}$ & $\begin{array}{l}\text { C243T } \\
\mathbf{n}(\%)\end{array}$ \\
\hline PUC (pure plasmacytoid) & $5 / 9(56)$ & $5(100)$ & -- & -- \\
\hline MPC (Pure micropapillary) & $18 / 18(100)$ & $15(83)$ & $3(17)$ & -- \\
\hline $\begin{array}{l}\text { Heterogeneous UC with focal } \\
\text { micropapillary features }\end{array}$ & $15 / 15(100)$ & $13(88)$ & $1(6)$ & $1(6)$ \\
\hline $\begin{array}{l}\text { Heterogeneous UC with focal } \\
\text { plasmacytoid features }\end{array}$ & $1 / 1(100)$ & $1(100)$ & -- & -- \\
\hline Total (n=43) & 91 & $34(79)$ & $4(9)$ & $1(2)$ \\
\hline
\end{tabular}

Conclusions: We found TERT promoter mutations, commonly found in conventional $\mathrm{UC}$, to be frequently present in MPC and PUC. Our finding of concordant intratumoral mutational alterations in cases with focal variant histology suggests a common cellular origin for both histologic components of this tumor type.
1005 A Reappraisal of Morphologic Differences between Renal Medullary Carcinoma (RMC) and Collecting Duct Carcinoma (CDC) after Exclusion of Newer Subtypes of Renal Cell Carcinoma (RCC): Report from the High-Grade Distal Nephron Adenocarcinoma (HDNA) International Consortium

Chisato Ohe. Cedars-Sinai Medical Center, Los Angeles, CA.

Background: A rare group of lethal high-grade, high-stage infiltrative glandular tumors of the kidney with desmoplasia, herein HDNA, includes previously known categories of RMC, CDC, \& RCC unclassified, and an emerging group of Fumarate Hydratase (FH)-deficient tumors of hereditary leiomyomatosis \& renal cell carcinoma syndrome (HLRCC). HDNA histomorphology has not been studied comprehensively across these groups to ascertain whether differential growth patterns can assist in classification. Design: 115 cases of HDNA were collected from 25 international institutions \& 10 countries (HDNA International Consortium), and were rigorously classified using contemporary histologic criteria and ancillary tests. The spectrum of morphologic patterns ( 7 in particular) was critically evaluated and compared between groups. Results: 115 HDNAs were classified as 33 RMC (INI1-, OCT4+/-, hemoglobinopathy), $40 \mathrm{CDC}$ (INI1+, OCT4-), 33 FH-deficient RCC (FH- and/or 2SC induced), and 9 unclassified RCCs (INI1-, hemoglobinopathy excluded). 10 (20\%) cases submitted as CDC were re-classified as FH-deficient RCC based on IHC and/or molecular studies. Comparing morphology between $\mathrm{CDC}$ and RMC, infiltrative glandular, cord-like, nested, sheet-like, and solid patterns were shared between both. Sieve-like, cribriform growth and reticular yolk sac-like growth favor RMC $(\mathrm{p}<0.05)$, whereas tubular/ papillary growth, multinodular infiltrating papillary growth, and intracystic papillary growth all favor CDC.

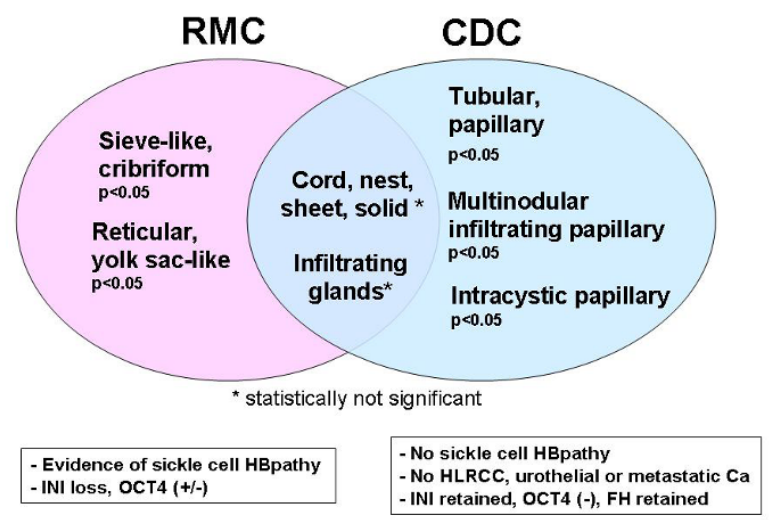

Conclusions: In addition to urothelial and metastatic carcinoma, FH-deficient RCC should be excluded before a diagnosis of CDC is rendered. Despite shared morphology of high grade infiltrative adenocarcinoma with desmoplasia, after rigorous characterization and exclusion of emerging RCC subtypes, intriguing, reproducible differences in morphology emerge between RMC and CDC. These features may be of prospective use for triage and classification of cases, empowering future studies of diagnostic \& predictive biomarkers.

1006 GSTM1, GSTT1, GSTP1 Polymorphisms, Quality of Life, Habits, Environmental Exposure and Predisposition to Bladder Cancer Isis Paloppi, Ruan CA Pimenta, Sabrina T Reis, Willian C Nahas, Miguel Srougi, Jose Cury, Mitiko Saiki, Katia RM Leite. University of Sao Paulo Medical School, Sao Paulo, Brazil; ICESP - Instituto do Cancer de Sao Paulo, Sao Paulo, Brazil; University of Sao Paulo, Sao Paulo, Brazil.

Background: The incidence of bladder tumor has increasing, possibly as a consequence of ongoing environmental pollution and poor personal habits. Previous studies have provided evidences that habits and trace elements are related to development of cancer. The glutathione S-transferases (GSTs) comprise a class of enzymes involved in the detoxification of DNA molecule result of insults exerted by environmental carcinogens. Our aim is to identify the relationship between habits, trace elements and genotype of GSTs with the development of bladder cancer.

Design: This is a case-control study were patients with bladder cancer were compared to healthy individuals. A questionnaire inquiring the habits was applied by a face to face interview. Deletion of GSTM1 and GSTT1 was identified by polymerase chain reaction (PCR) method and the genotype of two single-nucleotide polymorphisms of GSTP1 (rs1695 and rs1138272) was determined using real-time PCR (TaqMan). Serum trace elements ( $\mathrm{Br}, \mathrm{Ca}, \mathrm{Cl}, \mathrm{Fe}, \mathrm{Na}, \mathrm{Rb}, \mathrm{Se}$ and $\mathrm{Zn})$ were determined directly by using instrumental neutron activation analysis (INAA).

Results: Tobacco increased the risk of bladder cancer with an OR of $2.51(95 \% \mathrm{CI}$, 1.13 to 5.59$)$. People with bladder cancer reported eating more frying food $(\mathrm{p}<0.05)$ and less fish and fruit $(\mathrm{p}<0.05)$. Practice of physical activity seems to have a protective effect with an OR of $0.34(95 \%$ CI, 0.16 to 0.74$)$. There was a significant decrease of iron $(\mathrm{Fe})$ and chlorine $(\mathrm{Cl})(\mathrm{p}<0.05)$, and increased zinc $(\mathrm{Zn})$ in bladder cancer group $(p<0.05)$. GSTM1 null, GSTT1 null and polymorphisms of GSTP1 were not associated with bladder cancer risk.

Conclusions: We have shown that healthy alimentary habits and practice of physical activity can have a protective effect over the development of bladder cancer. The decrease of $\mathrm{Fe}$ and $\mathrm{Cl}$ and the increase of $\mathrm{Zn}$ are also related to an increase in bladder cancer development. 
1007 Muscularis Mucosae (MM) Thickness in Cystectomy Specimens: A Practical Objective Approach to Distinguishing from Muscularis Propria (MP)

Swetha Paluru, Jonathan I Epstein. Johns Hopkins Medical Institute, Baltimore, MD. Background: Identification of TUR specimens for MP invasion is critical as its identification typically leads to radical cystectomy. MP can be partially destroyed by tumor or fragmented such that it can be difficult to distinguish MP from MM. Smoothelin/desmin stains are not entirely specific and evaluation of the H\&E morphology remains the gold standard. This is the first study to assess the thickness of the MM in various locations in cystectomy specimens to determine the threshold of bundle thickness, above which would indicate MP.

Design: We identified 153 consecutive cystectomy specimens from 1/14-7/15. Cases where mucosa was disrupted or if the location of bladder wall section was not specified were omitted. The thickness of the entire MM in dome, lateral, posterior and anterior walls of the bladder was measured using an ocular micrometer at 10X magnification. Trigone was not assessed as it has a unique morphology.

Results: Of the 153 cases reviewed, 107 cases were acceptable. Patients' ages ranged from $48-84$ years (mean 68.7). 91 (85\%) were males. The MM ranged from 0.01 to 0.3 $\mathrm{mm}$. with a mean of 0.06 and median value of 0.05 .

\begin{tabular}{|l|l|l|l|l|}
\hline & Dome & Anterior & Posterior & Lateral \\
\hline Absent & 6 & 10 & 31 & 6 \\
\hline Mean & $0.08 \mathrm{~mm}$. & $0.05 \mathrm{~mm}$. & $0.05 \mathrm{~mm}$. & $0.06 \mathrm{~mm}$. \\
\hline Median & $0.06 \mathrm{~mm}$. & $0.03 \mathrm{~mm}$. & $0.04 \mathrm{~mm}$. & $0.05 \mathrm{~mm}$. \\
\hline Mininum & $0.01 \mathrm{~mm}$. & $0.01 \mathrm{~mm}$. & $0.01 \mathrm{~mm}$. & $0.01 \mathrm{~mm}$. \\
\hline Maximum & $0.3 \mathrm{~mm}$. & $0.3 \mathrm{~mm}$. & $0.25 \mathrm{~mm}$. & $0.25 \mathrm{~mm}$. \\
\hline
\end{tabular}

There was no significant difference in the thickness of MM between female and male patients or based on location. Five cases had a unique morphology located throughout the bladder, not previously described consisting of numerous closely packed bundles of MM separated by collagen measuring in toto on average $0.8 \mathrm{~mm}(0.5-0.1 \mathrm{~mm})$; the thickness of each bundle averaged $0.08 \mathrm{~mm}(0.03-0.1 \mathrm{~mm})$.

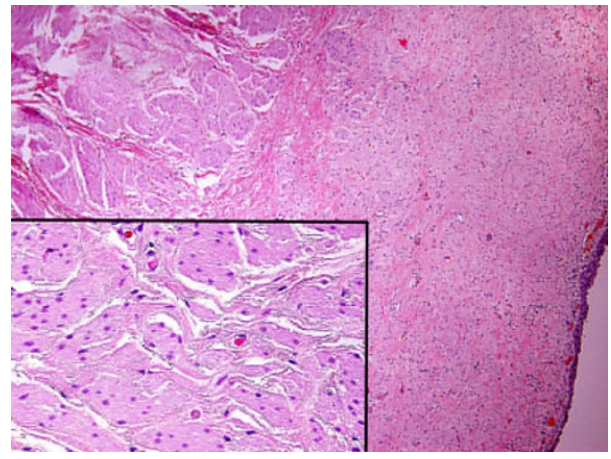

Conclusions: The muscularis mucosa measures to a maximum thickness of $0.3 \mathrm{~mm}$. This measurement can be used as a cut-off during the evaluation of muscle invasion in TUR specimens. A newly recognized mimicker is a variant of MM composed of closely packed MM bundles which if invaded by carcinoma could mimic MP invasion as in aggregate was thicker that typical MM.

1008 Morphologic Spectrum of Sertoliform Cystadenoma of the Rete Testis (SCRT): 14 Cases with Clinical Follow-Up

Swetha Paluru, Thomas M Ulbright, Mahul B Amin, Rodolfo Montironi, Jonathan I Epstein. Johns Hopkins Medical Institute, Baltimore, MD; Indiana University, Indianapolis, IN; Cedars-Sinai Medical Center, Los Angeles, CA; Polytechnic University of the Marche Region (Ancona), Ancona, Italy.

Background: SCRT is rare with only 6 cases reported to date, in the literature, none with follow up.

Design: Four large genitourinary pathology consult services were searched.

Results: We identified 14 cases of SCRT. Men were 21-84 years old (mean 43 years) and had testicular discomfort or mass. Other findings were seminoma $(n=1)$, spermatocele $(n=2)$, hydrocele $(n=1)$, varicocele $(n=1)$, and scrotal hematoma $(n=1)$. 8 had pre-operative serum tumor markers and were normal. Tumors ranged from 0.3 $\mathrm{cm}-4 \mathrm{~cm}$ (mean $1.5 \mathrm{~cm}$ ). All were well-circumscribed with solid and cystic features and occupied on average, $74 \%$ of the rete (20-100\%). The classic morphology (Figure, left) consisted of: 1) tubules without well-formed lumina $(n=12)$, mean $40 \%$ of tumor $(10-90 \%) ; 2)$ cords/nests with hyalinized or myxoid stroma $(\mathrm{N}=10)$, mean $26 \%$ of tumor (15-60\%); and 3) well-formed tubules $(\mathrm{N}=11)$, mean $17 \%$ of tumor $(5-70 \%)$. Other patterns included: 1) solid growth $(\mathrm{N}=4), 10-80 \%$ of tumor; 2$)$ individual cells $(\mathrm{N}=2)$, $5-10 \%$ of tumor; 3 ) festoons $(\mathrm{N}=2), 15-20 \%$ of tumor; 4$)$ branching tubules $(\mathrm{N}=1)$, $50 \%$ of tumor; and 5$)$ papillary $(\mathrm{N}=1), 30 \%$ of tumor. Cells were cuboidal with round to oval nuclei with small nucleoli, except at the periphery where projections into rete tubules had more columnar cells. In the festooning pattern (Figure, right), nuclei were pseudostratified and columnar with prominent nucleoli and nuclear grooves.

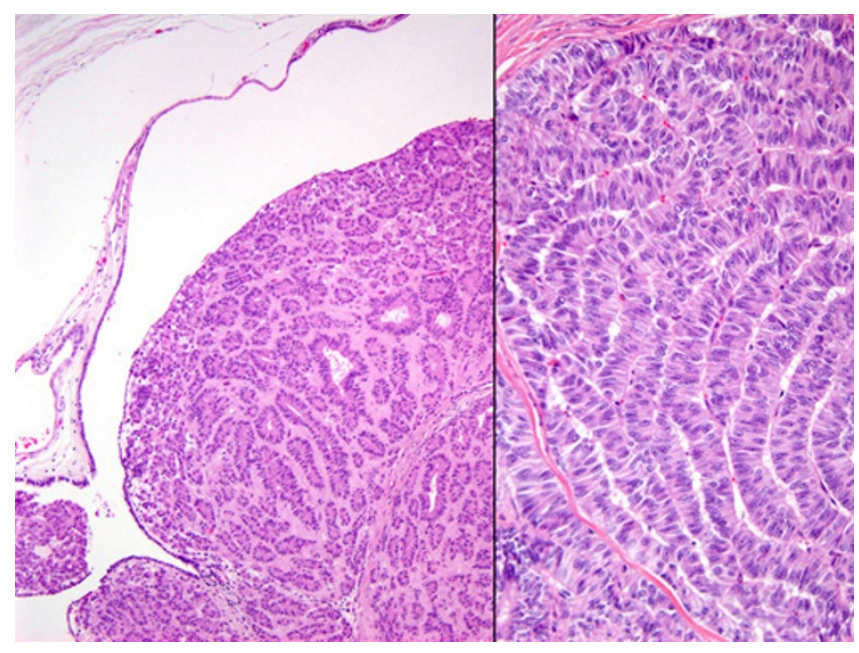

In 4 cases, tumor extended into adjacent seminiferous tubules surrounded by dense peritubular fibrosis with, in some cases, small cysts lined by flattened epithelium containing pale lightly granular material or whorls. All cases lacked necrosis and significant atypia. Mitoses ranged from 0-2 in $10 \mathrm{hpf}$. Follow up ranged from 4-170 months (median 114.5). For the 12 cases with information, all men were without evidence of disease except for 2 unrelated deaths, at 57 months and 10 years follow-up. Conclusions: This study highlights that SCRT has two distinct morphologies. We also verify the benign nature of the lesion and its lack of association with any syndromes.

1009 Whole-Exome Sequencing of Prostatic Stromal Sarcoma and Prostatic Stromal Tumor of Uncertain Malignant Potential

Chin-Chen Pan, Jonathan I Epstein. Taipei Veterans General Hospital, Taipei, Taiwan; Johns Hopkins Hospital, Baltimore, MD.

Background: The genetics of prostatic stromal tumor of uncertain malignant potential (STUMP) and stromal sarcoma are largely unknown. In previous study, we detected chromosomal aberrations in all stromal sarcomas and STUMPs. In this study, we performed whole-exome sequencing to detect somatic mutations in the prostatic stromal tumors.

Design: One stromal sarcoma from Taipei Veterans General Hospital, and 3 STUMPs (2 degenerative type, 1 myxoid type) from Johns Hopkins were used. Genomic DNA was extracted from the fomalin-fixed, paraffin-embedded tissues and constructed into library with Agilent SureSelect XT Reagent kit. The amplification adapter-ligated sample was purified using Agencourt AMPure XP beads. All samples were sequenced on Illumina Hiseq using 100PE protocol.

Sequence data were aligned to the reference human genome (hg19) using the BWA tool. Local realignment and quality score recalibration were performed using the GATK according to the best practices. SNP and Indel were called using UnifiedGenotyper and Varscan with default settings.

Results: After filtered by dbSNP-144, a total of 441, 436, 493 and 427 unreported nonsynonymous SNVs and indels were found in the stromal sarcoma and 3 STUMPs, respectively. Several candidate somatic mutations (GOLGA6L2, GXYLT1, HRNR, IGSF3, MCC, MUC2, NUDT11, OR2T35, OR6C76, PABPC1, RBMX, RPL14) occurred in high frequencies. TP53 mutations were found in 2 STUMPs. 141 mutations were detected exclusively in stromal sarcoma, not in any of the STUMPs. All cases harbored at least one of the following SNPs that have been reported as a risk factor for prostate cancer: TIMM23B (rs 10993994), RNASEL (rs486907), ELAC2 (rs4792311, rs5030739) and MUC6 (rs148581741).

Conclusions: Our study has identified several possible somatic mutations in prostatic stromal sarcoma and STUMP. More cases and biological studies are required to validate the significance of these genes in the oncogenesis of prostatic stromal tumors.

1010 Multicystic Chromophobe Renal Cell Carcinoma: A Challenging Unusual Architectural Variant. Morphological, Immunohistochemical and arrayCGH Analysis of 11 Cases

Maria Pane Foix, Ana Dunatov, Petr Martinek, Enric Condom-Mundo, Saul Suster, Maris Sperga, Jose I Lopez, Monika Ulamec, Stela Bulimbasic, Michal Michal, Kvetoslava Peckova, Kristyna Pivovarcikova, Ondrej Daum, Pavla Rotterova, Kristyna Kalusova, Milan Hora, Ondrej Hes. Bellvitge University Hospital, Barcelona, Spain; University Split, Split, Croatia; Medical Teaching School, University Hospital, Charles University, Plzen, Czech Republic; Medical College of Wisconsin, Milwaukee, WI; East University, Riga, Latvia; Cruces University Hospital, Barakaldo, Spain; University Hospital Zagreb, Zagreb, Croatia; Medical Teaching School, University Hospital, Charles University, Plzen, Czech Republic.

Background: Chromophobe renal cell carcinoma $(\mathrm{ChC})$ is typically composed of a combination of large leaf-like cells and smaller eosinophilic cells arranged in solid alveolar pattern. There were also described eosinophilic, adenomatoid/pigmented or neuroendocrine variants. We assembled 11 cases of $\mathrm{ChC}$ arranged in a distinct multicystic pattern.

Design: 11 multicystic $\mathrm{ChC}$ were selected out of $733 \mathrm{ChC}$ from our registry and subsequently analyzed by morphology, immunohistochemistry, arrayCGH, and FISH. 
Results: Patients were 8 males, 4 females with age range 50-89 years (mean 68.7). Size range was $1.5-20 \mathrm{~cm}$, mean $5.2 \mathrm{~cm}$. Follow up was available for 8 patients, range 1-19 years (mean 6.56). Tumors were composed of variable sized cysts and resembled multilocular multicystic clear cell RCC by architecture.

7/11 cases were composed exclusively of eosinophilic cells, 4 tumors of combination of leaf-like and smaller eosinophilic cells. Raisinoid nuclei were constantly present. All tumors were positive for EMA, CK7, OSCAR, CD117, parvalbumin, MIA and Pax 8; negative for vimentin, TFE3, CANH 9.

Multiple chromosomal losses were found in 2 cases out of 6 analyzable tumors, loss of chromosome 1 in one case and 3 tumors did not display chromosomal numerical aberrations.

Conclusions: $1, \mathrm{ChC}$ can be arranged in prominent multicystic pattern

2, Tumors show immunophenotype corresponding with classic $\mathrm{ChC}$.

3 , Chromosomal numerical aberration pattern was highly variable, resembling pattern of renal oncocytoma in 4 cases.

4, All cases showed an indolent, non-aggressive behavior.

1011 Spectrum of Cystic Epithelial Tumors of the Prostate: Most Cystadenocarcinomas (CACs) are Ductal Type with Intracystic Papillary Pattern

Gladell P Paner, Antonio Lopez-Beltran, Toyonori Tsuzuki, Jesse K McKenney. University of Chicago, Chicago, IL; Champalimaud Clinical Center, Lisbon, Portugal; Nagoya Daini Red Cross Hospital, Nagoya, Japan; Cleveland Clinic, Cleveland, OH. Background: Cystic epithelial tumors arising from prostate are rare and have only been the subject of case reports. The clinicopathological features of these unusual tumors have not been fully elucidated.

Design: 7 cystic prostatic tumors including 6 CACs and 1 cystadenoma (CA) from 4 institutions form the basis of this study.

Results: The 6 CACs were from men 47-82 yo (mean 66.5). Serum PSA was 3.5 to 171 $\mathrm{ng} / \mathrm{ml}$ (med 4.9). The cysts size varied: 3 were cystic pelvic masses (largest $7.7 \mathrm{~cm}$ ), 1 was thought as post-biopsy hemorrhagic cysts, and 2 were encountered as grossly visible cysts (up to $0.5-0.7 \mathrm{~cm}$ ) on pathological exam. The cystic mass presented with compressive symptoms while the small cysts were diagnosed from PSA elevation. Cyst fluid PSA in 1 CAC was $80,000 \mathrm{ng} / \mathrm{ml}$. Of 6 CACs, 5 showed ductal morphology with tall columnar cells and nuclear palisading. Nuclei were moderate to high grade. Papillae were common in all 5 ductal CACs and were florid in 4 tumors. Of 5 ductal CACs, 2 were pure and 3 were admixed with acinar adenoCa. In mixed histologies, ductal adenoCa predominated in the cystic component. $1 \mathrm{CAC}$ was pure acinar adenoCa and the cystic part showed focal papillae. All 6 CACs had non-cystic component composed of ductal $\& /$ or acinar adenoCa (GS $3+3$ to $5+4)$. All CACs showed racemase overexpression and mainly lacked basal cells by PIN4 staining. 1 tumor was pT3a,N1 and 4 were organconfined on RP. On follow-up (1-21 mos, med 7 mos), 1 died of metastasis to lymph nodes, liver and bone, and 4 had no evidence of disease. Review of 15 published CACs showed similar frequency of ductal adenoCa and occurrence of very high PSA in cyst fluid. The $1 \mathrm{CA}$ was from a 55 yo who had lower urinary tract symptoms attributed to a $10 \mathrm{~cm}$ cystic pelvic mass. The CA was lined by benign acinar and basal cells and focally by squamous epithelium. All 7 cystic tumors were positive for PSA.

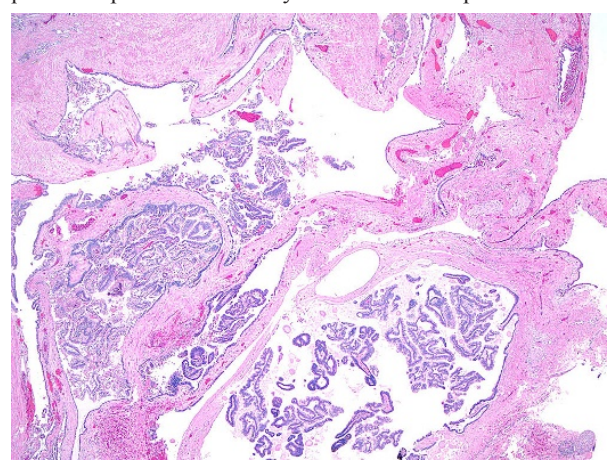

Conclusions: Cystic epithelial tumors of the prostate exhibit unique clinicopathological features. CACs should be recognized and considered for incorporation into future classifications of prostate neoplasms as a distinct variant.

\section{Novel and Rare Mesonephric-Type Tumors Arising from the} Urologic Tract

Gladell P Paner, Deepika Sirohi, Mukul Divatia, Mahul B Amin. University of Chicago, Chicago, IL; Cedars-Sinai Medical Center, Los Angeles, CA.

Background: Hyperplasia of mesonephric (Wolffian duct) remnant is well-documented in the prostate. However, the existence of mesonephric-type neoplasms primarily arising from the urologic tract has not been convincingly established.

Design: We present 3 cases, putatively of mesonephric-derived tumors involving the urinary bladder/urethra, prostate and seminal vesicle. Two cases were retrieved from the consultation files of the senior author and 1 case was retrieved from the surgical pathology files of a tertiary academic institution.

Results: Case 1: A 69 year old female underwent radical cystectomy for a $3.8 \mathrm{~cm}$ adenocarcinoma situated at the urinary bladder neck/urethra. The adenocarcinoma exhibited heterogenous patterns that included tubular, glandular, solid, focal papillary and focal spindled growth. Mesonephric differentiation was confirmed by the association of neoplastic tubules with luminal eosinophilic material that merged with benignappearing mesonephric remnants and positivity for Pax-8, CD10, CA125, calretinin, inhibin, WT1 and TTF1 (aberrant expression). There is no evidence of disease progression (7 months follow-up). Case 2: A 68 year old male underwent a transurethral resection of prostate for a moderately to poorly differentiated adenocarcinoma with tubular, glandular and retiform architecture. The tumor cells were cuboidal to occasional spindled cells with moderate atypia. Luminal spaces within the tumor contained eosinophilic material. Prostate histogenesis-related markers PSA, PSAP, PSMA, NKX3.1, prostein were negative. Mesonephric differentiation was confirmed by positivity for Pax-8, CD10, CA125 and MOC31. Case 3: A 22 year old male underwent resection of a $10 \mathrm{~cm}$ cystic mass that occurred in the left seminal vesicle. The tumor exhibited a biphasic histology with irregular sieve-like aggregates of cells and occasional secretions, the morphology being reminiscent of (female) adnexal tumor of probably Wolffian origin. There were also glandular and spindled to epithelioid cell areas. The neoplastic cells lacked pleomorphism but had brisk mitotic activity and high MIB1 proliferation index. Mesonephric differentiation was further confirmed by the positivity for Pax-8 and CD10; prostate histogenesis-related markers were negative. Conclusions: Malignant mesonephric-type tumors may rarely arise from adult prostate, seminal vesicles and the urinary tract. These rare Pax- 8 positive tumors should be included in the differential diagnosis of epithelial tumors with unusual morphology arising at these sites.

1013 Osseous Metaplasia in GU Organs: Pathogenesis \& Significance with First Case Report in Bladder Diverticulum

Rugvedita Parakh, Funda Vakar-Lopez, Maria S Tretiakova. University of Washington, Seattle, WA.

Background: Osseous Metaplasia (OM) is a rare phenomenon of uncertain clinical significance. We report $1^{\text {st }}$ case of $\mathrm{OM}$ in bladder diverticulum, which sparked further search of OM in genitourinary (GU) organs. We aimed our study to determine OM incidence in benign and malignant $\mathrm{GU}$ lesions, its morphology, clinical and prognostic significance based on our experience and literature search.

Design: 25 cases were retrieved from archives using key words "metaplastic, osseous /osteoid/ossification, dystrophic \& heterotopic" from 2006-2015 ( $\mathrm{N}=17)$ \& 3 months screening of current $\mathrm{GU}$ cases $(\mathrm{N}=8)$ (malignant bone formation was excluded). Literature search for OM yielded 25 case reports in kidney, 7 case reports \& 1 series in bladder $(\mathrm{N}=16)$. There were 11 case reports in penis, 3 reports in ureter/urethra, none in prostate \& adrenal. IHC for bone morphogenic protein (BMP) \& its receptor, sought to induce new bone formation, was also evaluated.

Results: OM in bladder diverticulum coexisted with squamous metaplasia \& squamous cell carcinoma suggesting urine stagnation and local irritation as pathogenic factors.

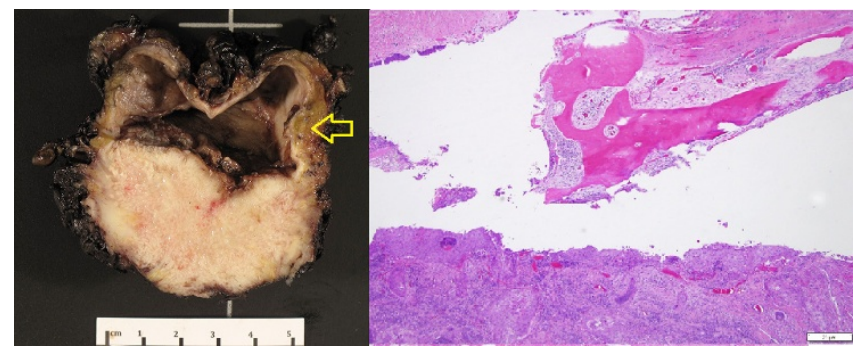

The most common site for OM in GU organs was kidney ( $40 \%)$, followed by bladder (24\%) \& penis (20\%), comparable to literature.Renal cell carcinoma (RCC), urothelial carcinoma (UC) and Peyronie's disease were most commonly associated with OM in our study \& literature.

\begin{tabular}{|l|l|l|l|l|}
\hline \multicolumn{3}{|c|}{ Our Institution cases } & \multicolumn{2}{c|}{ Published cases } \\
\hline Organ & $\mathrm{N}(\%)$ & Diagnosis & $\mathrm{N} \mathrm{( \% )}$ & Diagnosis \\
\hline Adrenal & $2(8 \%)$ & Myelolipoma 2 & 0 & -- \\
\hline Bladder & $6(24 \%)$ & $\begin{array}{l}\text { Carcinoma 4 } \\
\text { Inflammation 2 }\end{array}$ & $23(37 \%)$ & $\begin{array}{l}\text { UC 21 } \\
\text { Urachal Adenocarcinoma 2 }\end{array}$ \\
\hline Kidney & $10(40 \%)$ & $\begin{array}{l}\text { Clear cell RCC 7 } \\
\text { Oncocytoma 2 } \\
\text { Inflammation 1 }\end{array}$ & $25(40 \%)$ & $\begin{array}{l}\text { Clear cell RCC 10 } \\
\text { Chromophobe RCC 5 } \\
\text { Oncocytoma 3 } \\
\text { Renal Transplant 3 } \\
\text { Other 4 (B cell Lymphoma, papillary } \\
\text { adenoma, papillary RCC, Multilocular } \\
\text { Cystic RCC) }\end{array}$ \\
\hline Penis & $5(20 \%)$ & $\begin{array}{l}\text { Peyronie's 4 } \\
\text { Injury 1 }\end{array}$ & $11(18 \%)$ & $\begin{array}{l}\text { Peyronie's 10 } \\
\text { Injury 1 }\end{array}$ \\
\hline Prostate & $1(4 \%)$ & Brachytherapy & 0 & -- \\
\hline $\begin{array}{l}\text { Ureter/ } \\
\text { urethra }\end{array}$ & $1(4 \%)$ & Ureteric stone & $\mathbf{3 ( 5 \% )}$ & $\begin{array}{l}\text { UC 2 } \\
\text { Amyloidosis 1 }\end{array}$ \\
\hline Total & $\mathbf{2 5 ( 1 0 0 \% )}$ & & $\mathbf{6 2 ( 1 0 0 \% )}$ & \\
\hline
\end{tabular}

BMP2 and BMPR IHC in UC \& RCC with OM suggested their potential prognostic significance. Follow-up studies indicate negative prognostic impact of OM in UC patients, favorable for RCC patients.

Conclusions: We report $1^{\text {st }}$ cases of $\mathrm{OM}$ in bladder diverticulum \& prostate. The true incidence of $\mathrm{OM}$ in GU tract is expected to be much higher if reported consistently. Our study suggests that presence of OM has organ-specific significance with better outcomes in kidney RCC and worse outcomes in bladder UC. Preliminary BMP2 studies indicate OM pathogenetic \& prognostic role, and further understanding of its genesis. 
1014 FOXA1: A Transcription Factor Specific for Neuroendocrine Differentiation in Prostate Adenocarcinoma

Vamsi Parimi, Jung Kim, Jindan Yu, Ximing Yang, Maria M Picken. Loyola University Medical Center, Maywood, IL; Northwestern University, Chicago, IL.

Background: Prostate adenocarcinoma with neuroendocrine differentiation (PCND) association with androgen independence is well characterized. Androgen deprivation, Enzalutimide resistance, and cytokine induction have been suggested to induce neuroendocrine differentiation in prostate cancer. Current immunohistochemistry (IHC) neuroendocrine markers do not have high specificity for PCND diagnosis in diagnostic surgical pathology. We studied androgen independence role of FOXA1; Forkhead box A1 transcription factor specific for neuroendocrine differentiation in prostate adenocarcinoma (PCa) and its antibody specificity by immunohistochemistry in FFPE prostate tissue.

Design: LNCaP (+/-shFOXA1) and PC-3M (+/-shFOXA1) cell lines are used as specific dynamic cell line controls for the validation of

FOXA1 by IHC. A total of 50 poorly differentiated high Gleason grade $(>4+4) \mathrm{PCa}$ cases with and without PCND by H\&E morphology and or by IHC were stained for FOXA1 (Ab23738, Abcam), Chromogranin (DAK-A3, Dako), Synaptophysin (SY38 M0076, Dako) and NSE (22C9 NCL-NSE-435, Leica).

Results: FOXA1 positive nuclear staining is present in $100 \%$ of $\mathrm{PCa}$ and benign prostatic glands. Of $50 \mathrm{PCa}$ cases which showed neuroendocrine differentiation on hematoxylin and eosin (H\&E) stain, 10 cases showed diffuse positivity by all or any one of the neuroendocrine IHC markers. All 10 PCND cases of PCa showed no nuclear expression for FOXA1.

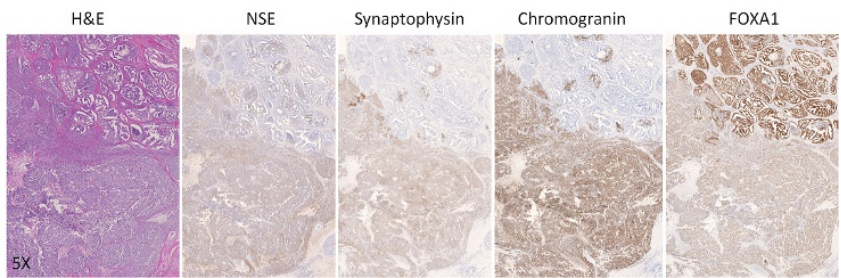

Conclusions: FOXA1 inhibits neuroendocrine differentiation. Absence of FOXA1 nuclear staining may be specific for PCND.

1015 Clinicopathologic Characteristics and Mutational Status of Succinate Dehydrogenase in Paraganglioma of Urinary Bladder with Emphasis on Malignancy: Multi-Institutional Study in Korea

Sanghui Park, Ghee Young Kwon, Ji Eun Kwon, Sang Kyum Kim, Ji Yeon Kim, Chul Whan Kim, Hyun-Jung Kim, Kyung Chul Moon, Yong Wook Park, Won Young Park, Eun Su Park, Ji Youn Sung, Sun Hee Sung, Seung Eun Lee, Wonae Lee, Jong Im Lee, Soo Jin Jung, Min-Sun Cho, Yong Mee Cho, Hyun Yee Cho, Eun Jung Cha, Yang Seok Chae, Gheeyoung Choi, Yeong Jin Choi, Jooryung Huh, Jae Y Ro. Ewha Womans University, Seoul, Korea; Other Hospitals, Korea; The Methodist Hospital, Houston, TX. Background: Paraganglioma of the urinary bladder is a rare neuroendocrine neoplasm, arising in extra-adrenal chromaffin cells of the autonomic nervous system and accounts for less than $0.05 \%$ of all bladder tumors. About 180 cases have been reported and less than 30 cases were malignant.

Design: To define clinicopathologic features and molecular characterization of succinate dehydrogenase (SDH) in a large cohort of paraganglioma of the urinary bladder, we performed a nationwide multicenter study with 19 major institutions in South Korea participated. Fifty two cases of paraganglioma from 1998 to 2014 were collected and their clinicopathologic features and $S D H$ mutational status were analyzed.

Results: There were 30 men and 22 women with a mean age of 56 (range, 22 - 79). Tumor size measured from 0.5 to $8 \mathrm{~cm}$ (mean, $2.4 \mathrm{~cm}$ ). Tumor necrosis was present in 5 cases $(10 \%)$, involvement of muscularis propria in 41 cases $(79 \%)$, and lymphovascular tumor invasion in 6 cases $(12 \%)$. During 41 months of mean follow-up period (range, 1-120), three cases (6\%) developed metastatic disease but none of them died of the disease. Among 3 patients with metastasis, metastatic sites were paraaortic lymph node $(\mathrm{n}=1)$, regional lymph node and bone $(\mathrm{n}=1)$, and multiple organ metastases with bone $(\mathrm{n}=1)$. Immunohistochemically, all cases showed intact SDHA (SDHA+). Among them, 43 cases were intact SDHB (SDHA+SDHB+), but 9 cases were deficient SDHB (SDHA+SDHB-). Compared to the SDHB-intact paragangliomas, the SDHB-deficient cases were characterized by a large tumor size (mean, $4.5 \mathrm{~cm}$ v.s $1.9 \mathrm{~cm}$ ), a higher mitoses per $10 \mathrm{HPF}$ ( 2.6 v.s. 0.1 ), lymphovascular invasion ( $33 \%$ v.s. $7 \%$ ) and frequent metastases ( 2 cases vs. 1 case). SDHB-deficient cases with metastases, compared to the SDHB-deficient cases without metastases, are characterized by a large tumor size (mean, $7.3 \mathrm{~cm}$ vs. $3.7 \mathrm{~cm}$ ), a higher mitoses per $10 \mathrm{HPF}$ ( $2.5 \mathrm{vs} 1.1$ ) and lymphovascular invasion (50\% vs. $29 \%$ ). SDHB, $S D H C$ and $S D H D$ mutational analyses were performed in nine SDHB-deficient cases, only six cases were successfully sequenced and all cases revealed only $S D H B$ mutations. Among $6 S D H B$-mutated cases, two developed metastases (33\%).

Conclusions: Our study demonstate that paraganglioma of the urinary bladder is a rare tumor with a $5.8 \%$ showing a malignant clinical course. A large tumor size, a higher number of mitoses, the presence of lymphovascular invasion and $S D H B$ mutation status suggest malignant behavior of paraganglioma.
1016 Clinical Utility of FISH for the Detection of Chromosome 3p Deletion in FFPE Tissue

Carmen M Perrino, Jason Orien, Weiqiang Zhao, Debra L Zynger. The Ohio State University Medical Center, Columbus, $\mathrm{OH}$.

Background: Deletion of chromosome $3 p$ is a well-established genetic alteration present in clear cell renal cell carcinoma (RCC). Ancillary testing with fluorescence in situ hybridization (FISH) for chromosome 3p deletion on formalin-fixed paraffinembedded (FFPE) tissue has been reported in research studies, but not in clinical practice. We aim to evaluate the clinical utility of $3 p$ FISH as an ancillary test in surgical pathology specimens.

Design: Detection of chromosome 3p loss by FISH on FFPE tissue was validated at our institution. FISH was performed using probes for 3pTel25 (spectrum green, Vysis TelVysion) and 3qTel05 (spectrum orange, Vysis TelVysion). A ratio (3p:3q signals) of $<0.8$ was established as the cut-off for $3 p$ loss. Testing was made available for clinical use for 2.5 years (2013-2015) and cases with 3p FISH performed were retrospectively analyzed. 2015 Medicare allowable billing rates were used to estimate the cost of ancillary studies.

Results: 3p FISH was performed at a rate of 18 cases per year and in 3.5\% (22/633) of institutional nephrectomies. Most cases submitted for $3 \mathrm{p}$ FISH testing were nephrectomy specimens $(69.8 \%, 30 / 43)$, of which $56.7 \%(17 / 30)$ were radical and $43.3 \%(13 / 30)$ were partial nephrectomies. 3 p loss was detected in $44.2 \%$ (19/43) of all tested cases. Radical nephrectomies had a higher rate of loss compared to partial nephrectomies $(70.6 \%$, $12 / 17$ vs $15.4 \%, 2 / 13 ; \mathrm{p}=0.003)$. Two-thirds of the cohort $(65.1 \%, 28 / 43)$ and $73.3 \%$ $(22 / 30)$ of nephrectomies were diagnosed with a definitive RCC subtype. Significantly more partial nephrectomies had a definitive RCC subtype assigned in comparison with radical nephrectomies $(92.3 \%$ vs. $58.8 \% ; p=0.04)$. The majority of nephrectomies with testing were diagnosed as clear cell RCC $(40.9 \%, 9 / 22)$ followed by clear cell papillary RCC $(31.8 \%, 7 / 22)$. Half of unclassifiable RCC had 3p loss $(8 / 15,53.3 \%)$, indicating that this finding was not sufficient to render a diagnosis of clear cell RCC. Annual 3p FISH costs were $\$ 3,446.64$. Other ancillary studies were frequently performed in the work-up of these cases with $78.5 \%$ of ancillary costs attributable to immunostains.

Conclusions: FISH for the detection of $3 p$ loss was performed infrequently in nephrectomy specimens. 3p FISH comprised a minority of the total cost of ancillary studies performed in these cases and therefore was not cost-prohibitive. Radical nephrectomies were more frequently positive for $3 p$ loss and partial nephrectomies were more frequently assigned a definitive RCC subtype. Despite detection of $3 p$ loss, some cases were not classified with an RCC subtype.

1017 Next Generation Sequencing (NGS)-based Molecular Characterization of High Grade Urothelial Carcinoma

Dinesh Pradhan, Leonard J Appleman, Rahul Parikh, Sheldon I Bastacky, Rajiv Dhir, Yuri E Nikiforov, Marina Nikiforova, Somak Roy. UPMC, Pittsburgh, PA.

Background: High-grade urothelial carcinoma (HGUC) of the urinary bladder is an aggressive disease with a high rate of morbidity and mortality. While advanced stage in general is associated with poor prognosis, there is considerable heterogeneity in clinical outcome, particularly with response to conventional chemotherapy. Recent studies, including the TCGA cohort, have attempted to elucidate the profile of HGUC; however there is still a paucity of published data to support routine clinical use of molecular analysis.

Design: Thirteen HGUC were retrieved from pathology archive at a large academic medical center after IRB approval. Formalin-fixed, paraffin embedded tissue sections were used for tumor microdissection. Extracted DNA was sequenced using a clinically validated Ion AmpliSeq Hotspot v2 panel (50 genes) on the Ion Torrent and Ion Proton platforms (Thermo Fisher Scientific Inc.). Bioinformatics analyses were performed using Torrent Suite v4.2 and custom developed software suite.

Results: Of the 13 HGUC, paired primary and metastatic tumors were sequenced in 7 cases. The mean age of the patients was 66.4 years $(47-88 \mathrm{yrs})$ and M: F ratio was 3.3:1. The mean tumor size was $4.6 \mathrm{~cm}$. Ten of the 13 cases had invasive UC, of which 8 had detrusor muscle invasion $(\geq \mathrm{pT} 2)$. Ten cases had nodal disease and 5 cases had distant metastasis (bone, lung and liver). Papillary morphology was present in 6 cases with variant histology (squamous and rhabdoid) in one case each. Genomic alterations in FGFR3, CDKN2A and TP53 were most frequent followed by PIK3CA (Figure 1). Additional mutations included ATM, KRAS, SMAD4, ERBB2, EGFR, AKT1, VHL and $10 \mathrm{q}(P T E N)$ loss. Besides papillary HGUC, FGFR3 mutation occurred in one case of flat HGUC (carcinoma in-situ). TP53 and CDKN2A alterations were frequent in detrusor muscle invasive UC. Interestingly, one case each harbored $E R B B 2$ and $E G F R$ amplifications in the metastatic focus only.

Conclusions: Molecular profiling of HGUC, particularly advanced or metastatic disease yields therapeutically actionable alterations such as FGFR3, ERBB2 and $E G F R$ as seen in our study, that can significantly impact clinical management of patients with therapy refractory HGUC. 


\begin{tabular}{|c|c|c|c|}
\hline FGFR3 & $46 \%$ & \multicolumn{2}{|c|}{ |ren } \\
\hline PIK3CA & $30 \%$ & a & 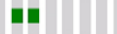 \\
\hline CDKN2A & $46 \%$ & & \\
\hline TP53 & $46 \%$ & - & mant \\
\hline ATM & $7 \%$ & 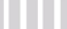 & - \\
\hline KRAS & $7 \%$ & 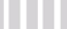 & घ \\
\hline SMAD4 & $7 \%$ & ( & - \\
\hline ERBB2 & $7 \%$ & the & 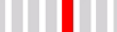 \\
\hline EGFR & $7 \%$ & & ( \\
\hline AKT1 & $7 \%$ & I & \\
\hline VHL & $7 \%$ & - & \\
\hline
\end{tabular}

1018 Utility of Immunohistochemistry for Distinguishing Luminal and Basal Subtypes of Urothelial Carcinoma on Formalin Fixed Paraffin Embedded Tissues

Dinesh Pradhan, Anthony Green, Kimberly A Fuhrer, Sheldon I Bastacky, Rajiv Dhir, Anil Parwani, Somak Roy. UPMC, Pittsburgh, PA; Wexner Medical Center, Columbus, $\mathrm{OH}$.

Background: High-grade urothelial carcinoma (HGUC) is an aggressive tumor with heterogeneous molecular and immunophenotypic profile and variable clinical outcome. Recent studies (PMID: 24525232) using gene expression profiling and RNA sequencing have described luminal and basal subtype of HGUC. The aim of this study was to assess the utility of the immunohistochemical (IHC) markers for distinguishing the two subtypes.

Design: 120 cases of HGUC, represented in 2 formalin fixed paraffin embedded tissue microarrays (TMAs) were used for this study. The TMAs were constructed using 200 cores to include various T stages of HGUC as well benign bladder tissue and appropriate orientation cores. Each tumor was represented with four-fold redundancy in these TMAs. The HGUCs were profiled using IHC that included 2 basal markers (Cytokeratin 14 \& Cytokeratin 5/6) and 2 luminal markers (GATA3 and FOXA1). Immunostains were evaluated using an $\mathrm{H}$-score-like method where a score of $\geq 200$ was considered as marker of protein overexpression.

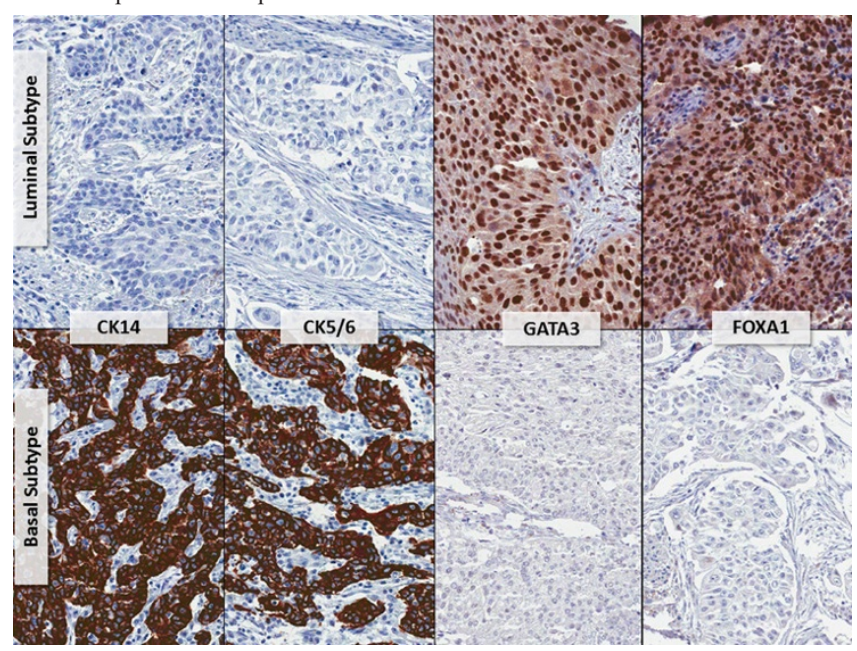

Results: Of the 120 cases, 107 cases had interpretable results. Based on immunoexpression profile, $20(18.7 \%)$ and $77(72 \%)$ cases were categorized as basal and luminal subtype, respectively. Notably, all of the basal tumors were muscle invasive in contrast to $61 / 77(79 \%)$ muscle invasive luminal subtype ( $<<0.05$, Fisher's exact). Squamous and sarcomatoid differentiation was enriched in the basal subtype $(9 / 20$ cases $45 \%)$ in contrast to luminal subtype $(5 \%)$ ( $p<0.0001$, Fisher's exact). Subtype classification did not show significant association with other clinicopathologic parameters. Ten $(9 \%)$ cases had overlapping immunoexpression of both basal and luminal markers and were difficult to classify. This may be attributed to tumor heterogeneity or may potentially belong to the p53-like subtype of HGUC previously described by gene expression profiling study (PMID: 24525232). Survival analysis with respect to subtype is currently under progress.
Conclusions: Subtyping of HGUC into basal and luminal subtypes on the basis of CK14, CK5/6, GATA3 and FoxA1 immunoexpression may be a cost-effective and useful ancillary tool for risk stratification of HGUC at the time of initial diagnosis.

\section{Variant Histopathology in Young-Age Prostate Cancer}

Susan Prendeville, Andrew Evans, Rob G Bristow, Theodorus $H$ van der Kwast. Laboratory Medicine Programme, University Health Network, Toronto, ON, Canada; Radiation Oncology, University Health Network, Toronto, ON, Canada.

Background: Prostate cancer ( $\mathrm{PCa}$ ) in young men represents an important clinical entity which has molecular genetic associations that are distinct from those found in elderly onset PCa. Data on the histopathologic features of cancers occurring in this cohort is limited. This study reviewed the histopathologic characteristics of prostate carcinoma in a series of men aged $\leq 45$ years.

Design: A retrospective search $(2001-2015)$ identified 62 prostatectomy cases with PCa in men aged $\leq 45$ years. Slide review was performed for all cases with Gleason score (GS) 7 or higher and a number of parameters were recorded including histopathologic subtype of carcinoma, pathologic stage and the presence/absence of unfavorable histologic features such as LVI and intraductal/large cribiform carcinoma.

Results: A total of $32 \mathrm{GS} \geq 7$ adenocarcinomas were identified. Median age was 43 years (range $39-45)$. Gleason score was $3+4(n=28), 4+3(n=3)$ and $4+5(n=1)$. Tumors were peripheral zone $(\mathrm{n}=17)$, transition zone $(\mathrm{n}=3)$ or $\operatorname{mixed}(\mathrm{n}=12)$ and multifocal $(\mathrm{n}=28)$ or unifocal $(\mathrm{n}=4)$. The tumor was classified as conventional acinar adenocarcinoma in 23 cases. A variant histopathologic subtype was identified in 9 cases, including 6 cases of mucinous carcinoma ( $>25 \%$ mucinous). All cases of mucinous carcinoma showed Gleason pattern 4 predominantly within the mucinous areas. Unfavorable histopathologic parameters were less common in mucinous tumors (see table 1). In addition, there were 3 cases with mixed acinar and ductal carcinoma.

\begin{tabular}{|l|l|l|l|l|l|}
\hline & & \multicolumn{3}{|l|}{ Histologic subtype } & Total \\
\hline pT stage & & $\begin{array}{l}\text { Conventional } \\
\text { Acinar (n=23) }\end{array}$ & $\begin{array}{l}\text { Mucinous } \\
\text { carcinoma } \\
(\mathrm{n}=6)\end{array}$ & $\begin{array}{l}\text { Mixed acinar } \\
\text { and ductal } \\
(\mathrm{n}=3)\end{array}$ & \\
\hline & $p T 2$ & $19(83 \%)$ & $6(100 \%)$ & $1(33 \%)$ & $26(81 \%)$ \\
\hline & $p T 3 a$ & $3(13 \%)$ & $0(0 \%)$ & $1(33 \%)$ & $4(13 \%)$ \\
\hline & $p T 3 b$ & $1(4 \%)$ & $0(0 \%)$ & $1(33 \%)$ & $2(6 \%)$ \\
\hline LVI & & & & & \\
\hline & Positive & 0 & $0(0 \%)$ & $1(33 \%)$ & $1(3 \%)$ \\
\hline & Negative & $23(100 \%)$ & $6(100 \%)$ & $2(66 \%)$ & $31(97 \%)$ \\
\hline $\begin{array}{l}\text { IDChlarge } \\
\text { cribiform }\end{array}$ & & & & & \\
\hline & Positive & $3(13 \%)$ & $0(0 \%)$ & $3(100 \%)$ & $6(19 \%)$ \\
\hline & Negative & $20(87 \%)$ & $6(100 \%)$ & $0(0 \%)$ & $26(81 \%)$ \\
\hline
\end{tabular}

Conclusions: Our series identified mucinous carcinoma in a significant proportion $(19 \%)$ of young-age ( $\leq 45$ years) prostate cancer cases, an uncommon histologic variant which accounts for $<1 \%$ of unselected cases. Further study is warranted to investigate the genetic aberrations associated with the mucinous phenotype in this young-age cohort and the associated clinical behaviour.

1020 Paratesticular Soft Tissue Lesions Identified in Orchiectomies Evaluated at a Single Tertiary Care Center

David Priemer, Karen Elizabeth Trevino, Thomas M Ulbright, Shaoxiong Chen, Muhammad T Idrees. Indiana University School of Medicine, Indianapolis, IN.

Background: Paratesticular neoplastic and non-neoplastic soft tissue lesions are rare but diverse. Many are found incidentally in orchiectomy specimens but some prompt it. There is no large demographic database for these lesions. Herein we document their frequencies with mean ages of presentation in orchiectomies at our institution. Design: We retrospectively searched our database of orchiectomy specimens from 1998 to $2015.4,741$ cases were reviewed. 137 were either performed for primary neoplastic or non-neoplastic lesions of the paratesticular soft tissue or showed an incidental paratesticular soft tissue lesion.

Results: The results are shown in the table. Of 137 cases, 97 (70.8\%) were neoplastic and the remaining non-neoplastic. The mean age of patients with neoplasms was 40.8 years (median: 35 ), and the majority of these were malignant $(62.8 \%)$. The mean age of patients with non-neoplastic disease was 41.3 years (median: 38.5 ). 
Table: Paratesticular soft tissue lesions

\begin{tabular}{|c|c|c|}
\hline \multicolumn{3}{|l|}{ Neoplasms } \\
\hline Diagnosis & Mean age(years) & Number of cases \\
\hline Rhabdomyosarcoma & 17.1 & 30 \\
\hline Spermatic cord lipoma^ & 41.3 & 27 \\
\hline Liposarcoma & 63.9 & 19 \\
\hline Hemangioma & 29.5 & 6 \\
\hline Leiomyosarcoma & 55.5 & 4 \\
\hline High-grade sarcoma, not otherwise specified & 73.3 & 3 \\
\hline Leiomyoma* & 43 & 2 \\
\hline Fibrosarcoma & 47 & 1 \\
\hline Myeloid sarcoma & 65 & 1 \\
\hline Osteosarcoma & 89 & 1 \\
\hline Synovial sarcoma (monophasic) & 60 & 1 \\
\hline Epitheliod sarcoma & 54 & 1 \\
\hline Cellular fibroma & 69 & 1 \\
\hline \multicolumn{3}{|l|}{ Non-neoplastic Lesions } \\
\hline Inflammatory and/or infectious ${ }^{\#}$ & 49.4 & 19 \\
\hline${\text { Adrenal rest }{ }^{\wedge}}$ & 30.2 & 16 \\
\hline Paratesticular necrosis/infarct & 23 & 2 \\
\hline Smooth muscle hyperplasia & 73 & 1 \\
\hline Heterotopic ossification & 50 & 1 \\
\hline
\end{tabular}

* One of the leiomyomas was designated as smooth muscle tumor of uncertain malignant potential

${ }^{\wedge}$ More commonly an incidental finding

"Diagnoses ranged from paratesticular abscess to chronic inflammatory reactions and inflammatory pseudotumor

Conclusions: In orchiectomy specimens, most paratesticular soft tissue lesions are neoplastic and the majority are malignant, most commonly rhabdomyosarcoma, liposarcoma, and leiomyosarcoma, the former, as expected, occurring at a significantly younger age. The most frequent benign neoplasms are spermatic cord lipoma and hemangioma. The most common non-neoplastic lesions are inflammatory and/or infectious processes or adrenal rests. Overall, there is no patient age difference between neoplastic and non-neoplastic soft tissue lesions of the paratestis.

1021 Characterization of Anterior Periprostatic Fat in a Large Cohort of Robotic-Assisted Radical Prostatectomy with Emphasis on Lymph Node Yield and Tumor Metastasis

Brian C Radlinski, Eric Rafla-Yuan, Peter E Clark, Giovanna A Giannico, Omar Hameed, Lan L Gellert. Vanderbilt University Medical Center, Nashville, TN.

Background: Anterior periprostatic fat (APF) is routinely excised to expose several landmark structures (e.g. puboprostatic ligaments and anterior surface of the dorsal vein complex) during radical prostatectomy. The presence of occasional lymph nodes (LN) has been described, but the necessity and significance of routine pathologic analysis of APF is still to be investigated. In this study, we systematically characterized the lymph node yield and tumor metastasis in a large cohort of robotic-assisted laparoscopic radical prostatectomy (RALP) and correlated the result with various clinicopathological factors. Design: 1042 consecutive cases of RALP with APF excision for biopsy proven prostate cancer from one single institution between 2012 and 2015 were included in this study. Pathology features of prostatectomy and APF were collected from pathology database, and clinical data were collected from patients' electronic medical record.

Results: Of the 1042 cases, lymph node(s) in APF were identified in 76 cases (7.3\%), and the lymph node count per case ranged from 1 to 3 . Pelvic lymph node dissection was performed in $332(32 \%)$ of 1042 cases, and $35(10.5 \%)$ exhibited nodal metastasis. No statistically significant association was identified between the presence of $\mathrm{LN}$ in APF and age $(\mathrm{p}=0.68)$, race $(\mathrm{p}=0.58)$, Gleason score $(\mathrm{p}=0.41)$, pathologic stage $(\mathrm{p}=0.10)$ or pelvic $\mathrm{LN}$ yield $(\mathrm{p}=0.16)$.

Of the 76 cases with LN in APF, 3 (4\%) exhibited nodal metastasis. All 3 cases exhibited Gleason score $>=7$ ( 8,7 and 7 respectively) in the radical prostatectomy, with stage pT3a for 2 and pT2c for 1 . One case also had metastasis in the pelvic LN. For the other 2 cases, no metastasis was identified in the pelvic $\mathrm{LN}$, and pathologic examination of $\mathrm{LN}$ in APF resulted in tumor upstaging.

Conclusions: Anterior periprostatic fat in RALP contains lymph nodes. Nodal metastasis present in those lymph nodes could result in tumor upstaging in a small portion of patients. These findings highlight the importance of routine pathologic examination of APF to ensure accurate tumor staging and proper clinical management.

1022 Inter-Observer Variability among Urologic Pathologists in the Grading of Penile Squamous Cell Carcinoma

Priya Rao, Benjamin Naovarat, Charles C Guo, Kanishka Sircar, Miao Zhang, Farah Khalil, Mike Hernandez, Pheroze Tamboli, Curtis A Pettaway, Philippe E Spiess, Jasreman Dhillon. MD Anderson Cancer Center, Houston, TX; Moffitt Cancer Center, Tampa, FL.

Background: Tumor grade in penile carcinoma is a critical pathologic finding that is often used as a surrogate tool to determine if inguinal lymph node resection is required in patients with no palpable lymphadenopathy. Tumor grade has been formally inducted into the 7th edition TNM classification for penile carcinoma to distinguish between pT1a and pT1b tumors. We wanted to examine the reproducibility of grading among urologic pathologists.

Design: 40 penile carcinomas were included in this study. 2 representative images from all 40 cases were distributed to 6 urologic pathologists from 2 institutes that specialize in the handling and reporting of penectomy specimens. Each pathologist was asked to assign a single grade of well (WD), moderate (MD) or poor (PD) for a particular case, based on their current practice.

Results: Consensus was defined as $4 / 6$ pathologists agreeing upon a single grade and was achieved in $28 / 40$ cases $(70 \%, \mathrm{k}$ value $=0.449)$. Concordance between all 6 pathologists was achieved in 9/40 cases; $22.5 \%$ (5 PD carcinomas, 2 MD carcinomas and $2 \mathrm{WD}$ carcinomas). The most easily identifiable pattern for a uniform diagnosis was the "basaloid" pattern which all pathologists classified as PD. Variants of WD carcinoma with abundant keratin production were also easily identifiable as WD. In 3 cases, all 3 grades were used for classification by the various pathologists, underscoring the difficulty in classification and poor reproducibility. Results are summarized in table 1.

\begin{tabular}{|l|l|l|l|}
\hline Outcome & Kappa & Z & Prob $>$ Z \\
\hline PD & 0.632 & 15.48 & $<0.001$ \\
\hline MD & 0.275 & 6.75 & $<0.001$ \\
\hline WD & 0.452 & 11.08 & $<0.001$ \\
\hline Combined & 0.449 & 15.37 & 0 \\
\hline
\end{tabular}

Conclusions: 1) The reproducibility in the grading of penile carcinoma even amongst urologic pathologists is moderate at best.

2) While the obviously PD \& WD carcinomas were easier to achieve consensus on, there was a wide range of opinions when tumors did not fit into either category

3) Better established guidelines are needed to help achieve uniform reporting of grade for these tumors

4) Since grading is integral for penile carcinoma staging (7th edition AJCC), stringent criteria need to be developed for pathologists to accurately classify the pT1b tumors based on the quantity of PD carcinoma.

1023 Prognostic and Pathologic Factors Determining Outcomes in pT2/ pT3 Penile Carcinoma:Time for a Revised Staging System

Priya Rao, Jasreman Dhillon, Benjamin Naovarat, Vipulkumar Dadhania, Xuemei Wang, Hsiang-Chun Chen, Philippe E Spiess, Curtis A Pettaway, Pheroze Tamboli. MD Anderson Cancer Center, Houston, TX; Moffitt Cancer Center, Tampa, FL.

Background: Tumors invading corpus spongiosum (CS) \&/or corpora cavernosa (CC) are currently staged as pT2 tumors. The aim of our study was to assess the 7th edition TNM pathologic staging system for penile carcinoma \& determine pathologic prognostic factors that determine clinical outcome.

Design: Our study includes 147 pts from 2 cancer institutes, with penectomies b/w June 1999 \& May 2013. Pts were stratified into 4 pathologic groups Group1: CS involvement without LVI ( $\mathrm{N}=41)$ Group2: CS involvement with LVI ( $\mathrm{n}=42)$ Group3: $C C$ involvement $(n=29)$ Group4: Urethra involvement $(n=35)$. Overall survival $(O S) \&$ disease free survival (DFS) were estimated using the Kaplan-Meier method, two-sided log-rank tests \& Cox proportional hazards regression models

Results: OS was significantly associated with tumor grade $(\mathrm{p}<0.001)$ \& pathologic group ( $\mathrm{p}=0.003$ when comparing grps $1-4 ; \mathrm{p}=0.002$ combining groups $1 / 2$ vs 3 or 4 ); median follow up $5.1 \mathrm{yrs}$, median DFS 1.5 yrs. Results are summarized in table 1.

\begin{tabular}{|l|l|l|l|l|}
\hline & Grp1 (n=41) & Grp2 ( $\mathrm{n}=42)$ & Grp 3 (N=29) & Grp4 ( $\mathrm{n}=35)$ \\
\hline 3 yr OS $(\mathrm{p}=0.003$ & $74 \%$ & $64 \%$ & $40 \%$ & $41 \%$ \\
\hline DFS ( $\mathrm{p}=0.002)$ & $65 \%$ & $38 \%$ & $34 \%$ & $33 \%$ \\
\hline
\end{tabular}

OS is significantly associated with histology grade $(\mathrm{HR}=0.49 ; \mathrm{p}=0.004) \&$ pathologic grp $(H R=0.55 ; p=0.01)$. On multivariate analysis of tumor grade (Grade $1 / 2$ vs Grade 3) \& pathologic grp (Groups 1,2,3,4 and groups 1-2 vs 3-4), DFS is significantly associated with pathologic group only.

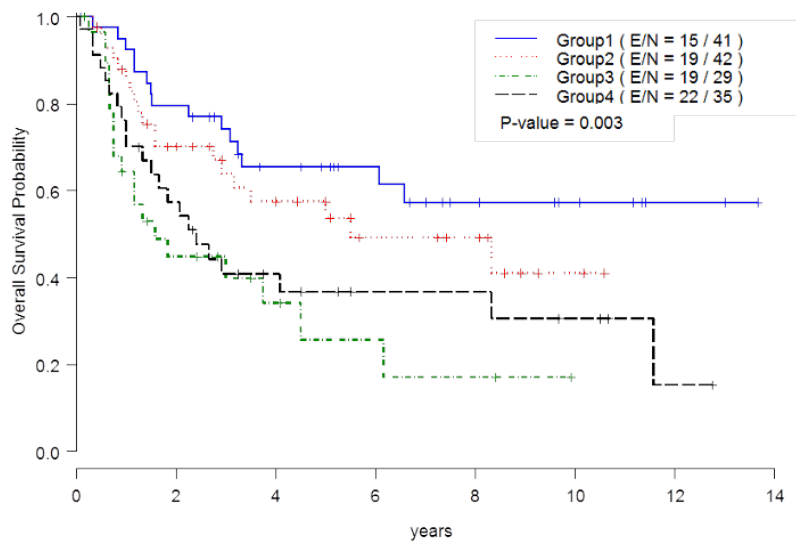




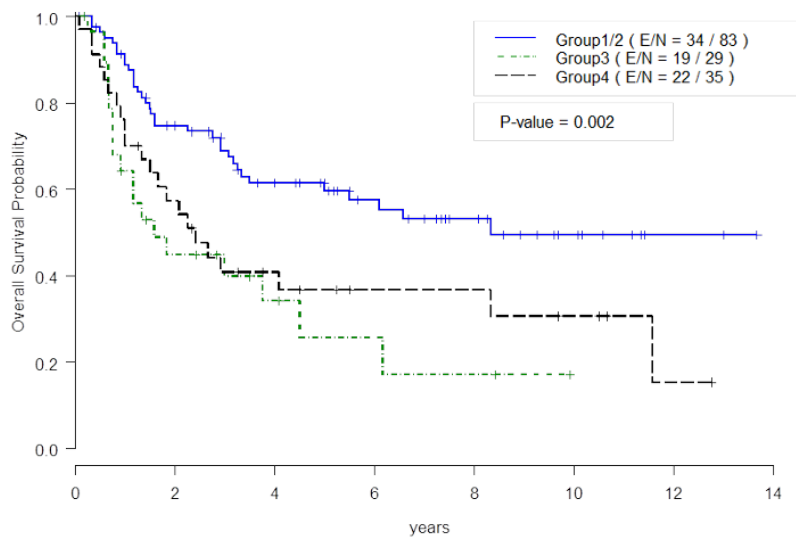

Conclusions: 1)There is a significant statistical difference in OS and DFS when tumors currently classified as pT2/pT3 tumors are stratified into 4 groups. 2) OS for tumors involving the $\mathrm{CC}$ is significantly worse than tumors involving the $\mathrm{CS}$, both with and without LVI \& behave similarly to tumors showing urethral involvement, thus warranting a separate T category 3 )On multivariate analysis, tumor grade did not significantly impact DFS in pT2 tumors, which was impacted by pathologic group alone

1024 Integrated Whole Exome and RNA Sequencing Reveals Distinct Genomic and Transcriptomic Landscape of Upper Tract Urothelial Carcinoma

Brian D Robinson, Bishoy Faltas, Rohan Bareja, Himisha Beltran, Joanna Cyrta, Peyman Tavassoli, Manoj Rai, Scott T Tagawa, David M Nanus, Francesca Khani, Juan Miguel Mosquera, Andrea Sboner, Douglas Scherr, Olivier Elemento, Mark Rubin. Weill Cornell Medical College, New York, NY.

Background: Upper tract urothelial carcinoma (UTUC) represents up to $10 \%$ of all urothelial carcinoma (UC). UTUC is a lethal malignancy, with nearly one half the patients dying within 5 years. Our objective was to understand the biological differences between UTUC and bladder UC.

Design: Fresh frozen chemotherapy-naïve primary tumors from nephroureterectomy cases and corresponding germline samples underwent whole exome sequencing (WES) and RNA sequencing (RNAseq). The Cancer Genome Atlas (TCGA) WES and RNAseq raw data was reanalyzed through our in-house bioinformatic pipeline to compare the mutational and transcriptomic landscape of UTUC to bladder UC. We evaluated the expression values for a set of 40 housekeeping genes between the two different datasets to exclude batch effects. We used gene set Enrichment Analysis (GSEA) to identify differentially enriched pathways in UTUC

Results: 17 tumors underwent WES, 20 RNAseq, with 11 analyzed for both WES and RNAseq. UTUC samples harbored several recurrent mutations including PIK3CA (4/17), FGFR3 (2/17), MLL2 (4/17), MLL3 (2/17), and ATM (2/17). Three KRAS mutations were discovered in two patients $(\mathrm{G} 12 \mathrm{D}, \mathrm{G} 12 \mathrm{~V}$ and $\mathrm{Q} 61 \mathrm{H})$, which were confirmed by targeted sequencing. Frequent copy number alterations included CDKN2A/B deletions (3/17), BG4ALT3, SEMG1 and USP6 amplifications (2/17 each). UTUC samples were characterized by differential lack of expression of the tumor suppressor ANP32D and overexpression of the cell cycle gene CDK10. GSEA analysis revealed significant enrichment of the KRAS signaling in UTUC whereas bladder UC showed an enrichment of genes involved in $\mathrm{mTORC} 1$ and $\mathrm{E} 2 \mathrm{~F}$ signaling. There were significant differences in the expression of several key DNA damage repair (DDR) pathway genes between the two entities including TP53, RAD51 and ERCC4 despite infrequent or absent mutations in these genes (FDR q value 0.03 for DDR gene set). MSH5, a gene associated with cisplatin-resistance was the most highly ranked DDR overexpressed gene in UTUC compared to bladder UC (enrichment score $=0.8$ ). Conclusions: This study generates a detailed genomic and transcriptomic profile of UTUC. Although morphologically identical and genomically similar, molecular differences between UTUC and bladder UC do exist. Importantly, RNA sequencing demonstrated a distinct pattern of DDR pathway expression in UTUC independent of genomic alterations. These findings may have important implications for platinum-based chemotherapy, and they provide a framework for understanding potential differences in the pathogenesis of UTUC versus bladder UC.

1025 Comprehensive Genomic Profiling of Urothelial Carcinoma of the Kidney and Ureter

Jeffrey S Ross, Julia A Elvin, Siraj M Ali, Jo-Anne Vergilio, James Suh, Tipu Nazeer, Vincent A Miller, Philip J Stephens. Albany Medical College, Albany, NY; Foundation Medicine Inc., Cambridge, MA.

Background: We compared the comprehensive genomic profiling results (CGP) for UC of the upper (UTUC) and lower (UCB) tracts to better understand the biology and to learn if opportunities and responses to targeted therapies would be similar for both diseases. Design: DNA was extracted from 40 microns of FFPE sections from 195 cases of relapsed/metastatic UTUC and 295 cases of UCB. CGP was performed on hybridizationcaptured, adaptor ligation based libraries to a mean coverage depth of $627 \mathrm{X}$ for up to 315 cancer-related genes plus 37 introns from 14 genes frequently rearranged in cancer. Genomic alterations (GA) included base substitutions (SUB), INDELs, copy number alterations (CNA) and fusions/rearrangements. Clinically relevant GA(CRGA) were defined as GA linked to drugs on the market or under evaluation in mechanism driven clinical trials.

Results: The UTUC cohort included 74 (38\%) female and $121(62 \%)$ male patients with a median age of 65.7 years (range 35 to 86 years). There were $174(89 \%)$ kidney and $21(11 \%)$ ureteral UTUC with $191(98 \%)$ high grade and $4(2 \%)$ low grade and with $6(3 \%)$ Stage II, 51 (26\%) Stage III and $138(71 \%)$ Stage IV tumors. Sequencing was performed on the primary tumor in $157(81 \%)$ of UTUC and from metastatic sites in $38(19 \%)$. For UTUC there were 1,243 total GA (6.4 GA/sample) involving 203 individual genes and $384 \mathrm{CRGA}$ (1.97 CRGA/sample) involving 51 genes. The most frequent GA were non-CRGA mutations in TP53 (46\%), CDKN2A (43\%), CDKN2B (36\%) and MLL2 (31\%). The most frequent CRGA in the UTUC patients:

\begin{tabular}{|l|l|l|l|l|l|l|}
\hline Gene & Sub & INDEL & CNA & Rearrangement & Splice & Total CRGA \\
\hline FGFR3 & 20 & 0 & 3 & 5 & 0 & 28 \\
\hline PIK3CA & 13 & 0 & 2 & 0 & 0 & 15 \\
\hline CCND1 & 0 & 0 & 12 & 0 & 0 & 12 \\
\hline MDM2 & 0 & 0 & 11 & 0 & 0 & 11 \\
\hline TSC1 & 7 & 2 & 2 & 0 & 1 & 11 \\
\hline ERBB2 & 5 & 2 & 4 & 0 & 0 & 11 \\
\hline
\end{tabular}

The CRGA in FGFR3 (28\% vs 21\%), PIK3CA (15\% vs $20 \%)$ and total ERBB2 $(11 \%$ vs $16 \%$ ) are similar in UTUC vs UCB. Of the 14 UTUC with ERBB2 non-amplification alterations, $6(43 \%)$ featured a micropapillary growth pattern similar to that seen in UCB (40\%). Clinical responses of UTUC to MTOR, FGFR and ERBB2 inhibitors will be presented.

Conclusions: CGP of UTUC reveals frequent targetable GA in FGFR3, PIK3CA and $E R B B 2$ and only minor differences in CRGA when compared to those found in the more common UCB indicating that targeted therapy opportunities for both diseases are similar. Further study of targeted therapy for genomically characterized UTUC in a clinical trial setting appears warranted.

\section{Lymphomas of the Urinary Tract and Genital Organs: An} Institutional Study of $\mathbf{5 0}$ Cases

Julia A Ross, Pei Hui, Peter A Humphrey, Adebowale Adeniran. Yale University School of Medicine, New Haven, CT.

Background: Lymphomas of the urinary tract and genital organs are rare lesions, and only a handful of small case series and isolated case reports have been published. Few studies have examined the overall frequency and specific subtypes of genitourinary lymphomas. This study sought to determine the overall frequency and subtype frequency of lymphomas affecting the urinary tract and genital organs at a single institution, as well as identify specific organs predominately involved by lymphoma.

Design: All cases of urinary tract and genital organs with the diagnosis of lymphoma at our institution between 1995 and 2015 were retrospectively reviewed. H\&E slides and immunohistochemical stains were reviewed. Demographic data, tumor characteristics, molecular and flow cytometry data (if available) were obtained from the reports. Cases with involvement of pelvic lymph nodes, without parenchymal involvement were excluded from the study.

Results: A total of 50 cases were identified. This included 22 female and 28 male patients (age range 10-83 years; median 59 years). 36 cases $(72 \%)$ were primary extranodal genitourinary tract lymphomas. Diffuse large B cell lymphoma (DLBCL) was identified in 25 cases $(50 \%)$, chronic lymphocytic leukemia/small lymphocytic lymphoma (CLL/ SLL) in 5 cases $(10 \%)$, B-cell lymphoblastic leukemia/lymphoma (BLL) in 3 cases (6\%), T cell lymphoma (TCL) in 3 cases $(6 \%)$, marginal zone B cell lymphoma in 2 cases (4\%), Burkitt lymphoma (BL) in 2 cases (4\%), post transplant lymphoproliferative disorder (PTLD) in 2 cases (4\%), low-grade B cell lymphoma, NOS in 2 cases (4\%) and 1 case each of mantle cell lymphoma, T cell lymphoblastic leukemia (TLL), follicular lymphoma, Richter's transformation, granulocytic sarcoma, and Burkitt-like lymphoma ( $2 \%$ each). Testis was the most common site (14 cases, $28 \%$ ), followed by kidney (11 cases, $22 \%$ ), ovary ( 10 cases, $20 \%$ ), vagina/cervix/mons pubis ( 7 cases, $14 \%$ ), prostate ( 4 cases, $8 \%$ ), uterus ( 2 cases, $4 \%$ ), and bladder ( 2 cases, $4 \%$ ). Associated concurrent secondary malignancy of the genitourinary tract was found in 3 cases.

Conclusions: Although rare, consideration of a lymphoma in the genitourinary tract is important for proper diagnosis and treatment of these lesions. The diversity of lymphoma subtypes and sites of involvement in this study underscores the importance of considering lymphoma in the differential diagnosis of genitourinary tract tumors and fully characterizing them by immunohistochemistry and other ancillary studies.

1027 High Grade Renal Cell Carcinoma with Emperipolesis. Clinicopathological, Immunohistochemical and Molecular-Genetic Analysis of 14 Cases

Pavla Rotterova, Petr Martinek, Kristyna Kalusova, Ivan Damjanov, Saul Suster, Isabel Alvarado-Cabrero, Maris Sperga, Marian Svajdler, Kvetoslava Peckova, Kristyna Pivovarcikova, Ondrej Daum, Milan Hora, Michal Michal, Ondrej Hes. University Hospital Plzen, Charles University, Plzen, Czech Republic; University of Kansas, Kansas, KS; Medical College of Wisconsin, Milwaukee, WI; Centro Medico, Mexico City, Mexico; East University, Riga, Latvia.

Background: Emperipolesis has been described recently as a constant feature of "biphasic alveolosquamoid" renal cell carcinoma (BARCC). We undertook the present study to determine the incidence of emperipolesis in high grade (HG) RCC. 
Design: 14 cases of HGRCC showing emperipolesis were retrieved from our registry of 18500 renal tumors. Microscopic examination of filed slides was supplemented with immunohistochemical and molecular-genetic data obtained from the study of paraffin blocks.

Results: There were 14 patients: 12 males, 2 females, all in the age group of 41-72 years (mean 58.64 years). Tumor size varied from 6 to $16.5 \mathrm{~cm}$, with a mean of 8.67 $\mathrm{cm}$. Follow up ( $0.5-10$ years, mean 4.28 , median 10 years) was available for $8 / 14$ patients. Metastases were documented in 6 cases; 4 patients died of disease $(0.5-10$ years after diagnosis); 1 is alive with metastases to the lung 7 years; 1 is alive 0.5 year after nephrectomy. All tumors appeared solid-alveolar with focal pseudopapillary features and were composed of large cells with bizarre nuclei (grade 4) and eosinophilic rhabdoid cytoplasm. Emperipolesis was a constant and prominent feature.

All cases were positive for OSCAR, CANH 9, vimentin, cyclin D1, INI-1, myoD1, negative for melanocytic markers, CK 7, myoglobin, cathepsin K, TFE3.

VHL gene abnormalities were found in 6/9 analyzable cases. In two cases with a $V H L$ abnormality there was a polysomy of chromosomes 7,17 .

Conclusions: 1. All 12 cases fulfilled the morphologic and immunohistochemical criteria for diagnosis of HG clear cell RCC, however 2 tumors had the molecular-genetic features of both HG clear cell RCC and HG papillary RCC.

2. Metastases were documented in $6 / 8$ cases

3. Emperipolesis is a rare change and besides BARCC it can be seen in other RCC, mostly in HG clear cell RCC.

1028 Chromosomal Abnormalities of Tumors with Features of Mucinous Tubular and Spindle Cell Carcinoma of the Kidney yet with High Grade Cytology

Evita T Sadimin, Yingbei Chen, Pedram Argani, Jonathan I Epstein. Rutgers Cancer Institute of New Jersey, New Brunswick, NJ; Memorial Sloan Kettering Cancer Center, New York, NY; Johns Hopkins Medical Institutions, Baltimore, MD.

Background: Mucinous tubular and spindle cell carcinoma (MTSCC) of the kidney is a distinct entity included in the 2004 World Health Organization (WHO) Classification of Tumours and the more recent International Society of Urological Pathology Vancouver Classification of Renal Neoplasia. Generally, it is characterized by bland tightly packed elongated tubules and spindle cells with low nucleolar grade in a basophilic myxoid stroma. In most cases, it is associated with indolent behavior. Several case studies have reported MTSCC with high grade features and have brought into question whether they represented MTSCC or a variant of papillary renal cell carcinoma.

Design: We searched our pathology database for unclassified renal cell carcinoma with histologic features of MTSCC, yet had higher grade cytological atypia. Six cases of renal cell carcinoma with features of MTSCC with high nucleolar grade (focal in two cases, diffuse in four cases) were identified. We identified an additional case with overall low nucleolar grade but with extensive necrosis. DNA samples were extracted from paraffin blocks of all seven cases were analyzed using a SNP array platform.

Results: Six out of seven patients were female. The ages ranged between 46 to 82 years with sizes ranging from 3 to $7.5 \mathrm{~cm}$. One case showed involvement of renal sinus fat and a second case showed involvement of the perinephric fat. Three cases had necrosis. All our cases shared common chromosomal abnormalities observed with the more typical MTSCC, with monosomy of chromosome 1,4,6,8,9,13,14,15 and 22. Some of the cases had additional gains/losses or copy number-neutral loss of heterozygosity (CN-LOH). Trisomy 7 or 17 were not observed in any of the cases. Follow up interval was between 1 and 9 years. None of the patients showed evidence of recurrence or metastasis.

Conclusions: The molecular analysis performed in this study supported that MTSCC of the kidney can have high nucleolar grade or extensive necrosis, and that they are not a variant of papillary renal cell carcinoma. Thus far, based on our study and most of previous studies, MTSCC with high grade features are still associated with a good prognosis, though the case number and follow up of our study were limited. Increased recognition of this entity will hopefully lead to additional studies to further assess its behavior.

\section{Interobserver Reproducibility of Percentage Gleason Pattern 4 in} Prostatic Adenocarcinoma

Evita T Sadimin, Francesca Khani, Mairo L Diolombi, Abdelrazak Meliti, Jonathan I Epstein. Johns Hopkins Medical Institutions, Baltimore, MD.

Background: At the 2014 International Society of Urological Pathology (ISUP) consensus conference on grading in prostatic adenocarcinoma, it was recommended that the percent of pattern 4 be reported on pathology reports to better reflect the extent of pattern 4 in Gleason score 7 tumors.

Design: We analyzed prospectively 422 cores containing Gleason pattern 4 from our consult cases in a period of 3 months. The percentage of pattern 4 was assigned to all the cases in $10 \%$ increments from $0-100 \%$ (with the addition of $5 \%$ ) by one of the four fellows in urologic pathology and by a more experienced urologic pathologist. Kappa statistics were performed: (0.41-0.60 - Moderate Agreement; 0.61-0.80 - Substantial Agreement)

Results: Out of 422 cores, $32 \%$ was an exact match and $75 \%$ was within $\pm 10 \%$ (weighted kappa value 0.67 ). The cases were then re-analyzed for the following: (A) clustered pattern 4 versus scattered pattern 4 in the background of pattern 3 , (B) cribriform pattern only versus poorly formed/fused pattern versus mixed cribriform and poorly formed/ fused pattern, and $(\mathrm{C})$ size of cancer $(\leq 10 \%$ versus $>10 \%$ of the core). No significant difference was observed in (A) and (B). However, in cases with $\leq 10 \%$ involvement of the core, $61 \%$ was within $\pm 10 \%$ (weighted kappa value 0.50 ) compared to cases with $>10 \%$ involvement of the core, where $78 \%$ was within $\pm 10 \%$ (weighted kappa value 0.70 ). Conclusions: Our study analyzed the reproducibility of reporting percentage Gleason pattern 4 among urologic pathologists that have been trained to use the same criteria as an expert pathologist. We showed that while it was relatively reproducible with substantial agreement within $\pm 10 \%$ in cases with $<10 \%$ involvement of the core, it was more difficult to assess in smaller foci, with only moderate agreement. Given that in a small focus only a few glands of a given pattern can markedly affect the percent pattern 4, consideration should be given to not recording percent pattern 4 in small foci of Gleason score 7 cancer on needle biopsy.

\section{Clinicopathological Characteristics of Patients with Fungal} Prostatitis

Evita T Sadimin, Melanie Parikh, Daniel Luthringer, Deepika Sirohi, Mahul B Amin, Jonathan I Epstein. Rutgers Cancer Institute of New Jersey, New Brunswick, NJ; Cedars-Sinai Medical Center, Los Angeles, CA; Johns Hopkins Medical Institutions, Baltimore, MD.

Background: Fungal prostatitis is a rare entity with the literature mostly composed of case reports.

Design: We searched the pathology databases of two institutions for patients with fungal prostatitis. All the slides were retrieved and reviewed.

Results: Ten patients were identified, ranging from 53 to 79 years old. Organisms identified included Cryptococcus spp. $(\mathrm{n}=4)$, Coccidioides $\mathrm{spp} .(\mathrm{n}=5)$, and Histoplasma $\mathrm{sp} .(\mathrm{n}=1)$. Five patients were immunocompromised, including one with cryptococcal meningitis. The other five patients were immunocompetent, two of whom were diabetic. One of the diabetic patients had lung involvement by Coccidioides. Two patients with Cryptococcus had known exposure. The clinical presentation varied: elevated prostate specific antigen $(n=5)$, abnormal digital rectal examination $(n=2)$, and increased urinary urgency and frequency $(n=3)$. Histologically, all cases showed granulomatous inflammation surrounding the organisms, with varying degrees of acute and chronic inflammation in other areas of the prostate. In contrast to nonspecific granulomatous prostatitis, the granulomas were identified in a peri-glandular distribution without being a reaction to ruptured glands. In the cases with Cryptococcus and Coccidioides, the granulomas were associated with caseating necrosis while in the case with Histoplasma no necrosis was present. All organisms were best visualized with Grocott's Methenamine Silver staining. Additionally, mucicarmine stain intensely highlighted the capsule of Cryptococcus. All patients with available outcome information were treated with antifungal medications with resolution of disease, except in one of the more severe cases where the patient eventually had a radical prostatectomy. In another severe case, the prostate became fibrotic and scarred after treatment, leading to persistent lower urinary tract symptoms.

Conclusions: Fungal prostatitis should be considered in the differential diagnosis when peri-glandular granulomatous inflammation is present in the prostate surrounding generally intact acini, in both immunocompromised and immunocompetent patients. Most cases diagnosed in prostate biopsies can be successfully treated; however a small subset may have extensive disease with long term sequlae or need for surgical intervention underscoring the importance of accurate histologic recognition.

\section{Testicular Sex Cord-Stromal Tumors of Unclassified Type}

Omer AM Saeed, Thomas M Ulbright, Muhammad T Idrees. Indiana University School of Medicine, Indianapolis, IN.

Background: Sex cord-stromal tumors (SCSTs) of the testis represent only 5\% of all testicular neoplasms. Among them, unclassified SCSTs represent a rare and poorly characterized group of tumors. The morphologic spectrum of this group is not comprehensively documented. The objective of this large series of unclassified SCSTs was to evaluate the clinical features, morphologic spectrum, and behavior of these neoplasms.

Design: An electronic data search of our institution's files was performed for consecutive surgical cases diagnosed as unclassified SCSTs from 1998-2015. The surgical reports were evaluated for morphology and the electronic records were examined for demographic and clinical data

Results: Twenty-nine cases were identified, including 26 consultations and 3 inhouse. All but 6 patients were white. Ages were 6 months to 66 years (mean 33.9; SD 18.1). The most common presentation was a testicular mass. Tumor sizes ranged from $0.2-11 \mathrm{~cm}$ (mean 3.4; SD 2.8). Of the tumors, 39\% met previously published histologic criteria for malignancy, while $39 \%$ were morphologically benign; $21 \%$ had only one feature worrisome for malignancy, and these were categorized as neoplasms of borderline malignant potential. The tumors showed variable histologic patterns. Most were arranged in nests and groups of round to ovoid to spindle cells without prominent background stroma. About one-third showed some Sertoli cell differentiation but without overt tubule formation. A prominent spindle cell component was found in $17 \%$, while a few cases showed granulosa, myogenic, or clear or squamous cell differentiation. The malignant cases were larger (malignant: mean 5.3; SD 2 and benign: mean 1.4; SD 0.9) and had higher mitotic activity, necrosis, and nuclear atypia; $36 \%$ showed lymphovascular invasion. Tumor cells demonstrated some variability with immunostaining. Immunostaining for calretinin, inhibin, and S100 was positive in $60 \%$, $82 \%$, and $50 \%$ of the cases, respectively. Follow up of 8 patients showed metastatic disease in 3 . All 3 were originally diagnosed as malignant.

Conclusions: Unclassified SCSTs are unique tumors with a wide spectrum of morphologic features without a specific pattern of differentiation. Approximately $60 \%$ showed histological features of malignancy or malignant potential. They are diagnostically challenging and immunostains may prove unhelpful in diagnosis, making familiarity with their morphology critical. Accurate classification into the sex cord-stromal category is essential for proper management 
1032 Metabolomics Analysis Reveals Distinct Profiles of Non-Muscle Invasive and Muscle-Invasive Bladder Cancer

Divya Sahu, Yair Lotan, Bryan Wittman, Bruce Neri, Donna Hansel. University of California at San Diego, San Diego, CA; Metabolon Inc, Durham, NC; University of Texas Southwestern Medical Center, Dallas, TX.

Background: Only limited metabolomics studies, which provides a comprehensive metabolite signature of biological activity within a tissue, have been performed on bladder cancer specimens. We utilized metabolomics to identify differential pathway activity, and thus potential biomarker and therapeutic opportunities, in normal urothelium, non-muscle invasive urothelial carcinoma and muscle-invasive urothelial carcinoma.

Design: We analyzed 24 benign urothelium, 48 high-grade non-muscle invasive urothelial cancer and 48 muscle-invasive urothelial cancer specimens using global metabolomics profiling, coupled with publicly available data on transcriptomics of key enzymes, to determine pathways dysregulated in bladder cancer. Metabolomics profiling was performed at Metabolon Inc, where extracts of all samples were split for analysis by Liquid Chromatography Mass Spectrometry (LC/MS) and by Gas Chromatography Mass Spectrometry (GC/MS). Compounds were identified by comparison to library entries of purified standards or recurrent unknown entities. Statistical analyses were performed with one or both of the statistical analysis software programs: Array Studio (Omicsoft, Inc) or "R" http://cran.r-project.org/.

Results: Categorical pathways globally dysregulated in cancer relative to benign urothelium included glucose, TCA cycle, lipid, amino acid and nucleotide pathways. Bladder cancers demonstrated Warburg metabolism, with elevated glucose utilization to drive glycolysis and sorbitol pathway intermediates. Elevated late TCA cycle intermediates, coupled with higher levels of amino acids and dipeptides, suggest the possibility of anaplerotic activity in bladder cancer as a mechanism to sustain energy production. Medium and long chain fatty acids were produced at the expense of dicarboxylic fatty acids. Muscle-invasive bladder cancers showed enhanced use of inflammatory regulation and modification of NAD+ synthesis in muscle-invasive bladder cancer. Transcriptomic profiling suggests that the majority of metabolomics pathway alterations correspond to gene expression changes of enzymes responsible for metabolite production.

Conclusions: This study identifies multiple parallel metabolomics changes unique to non-muscle invasive and muscle-invasive bladder cancer that can be used to justify testing putative predictive biomarkers of progression and novel therapeutics targeting metabolomics pathways in bladder cancer.

1033 Papillary Renal Cell Carcinoma Is More Than One Entity. Molecular Subtyping and Pathway Analysis

Rola Saleeb, Pamela Plant, Eriny Tawedrous, Adriana Krizova, Sahar Al-Haddad, Andrew Evans, Fadi Brimo, Samantha J Wala, George M Yousef. University of Toronto, Toronto, ON, Canada; St. Michael's Hospital, Li Ka Shing Knowledge Institute, Toronto, ON, Canada; Toronto General Hospital, Toronto, ON, Canada; Montreal General Hospital, McGill University, Montreal, QC, Canada.

Background: Papillary Renal Cell Carcinoma (PRCC) is the $2^{\text {nd }}$ most common type of Renal Cell Cancer. It is recognized to have 2 morphological subtypes. Previous studies have shown the two types to differ in prognosis and in characteristic genetic alterations. Our study aims to elucidate the molecular differences between the subtypes on the genetic, and epigenetic level; giving insight into the implicated molecular pathways Design: PRCC, 291 patient cohort, of the Cancer Atlas Genome (TCGA) was divided on histological basis into type 1, type 2 or non-otherwise specified (NOS). Gene expression (RNA seq) data for the cohort was examined using unsupervised clustering, and supervised clustering bioinformatics analysis. A total of 35 primary PRCC specimens were also collected, for miRNA assessment, after similar morphological classification into different subtypes. Gene set enrichment analysis (GSEA) was performed on both cohorts.

Results: Analysis of gene expression and miRNA expression between the subtypes using unsupervised clustering revealed two distinct genetic tumor signatures. Supervised clustering produced statistically significant identifying markers for each subtype. GSEA revealed considerably different pathways implicated in each type including VEGF, mTOR , P53 and Adherens pathways.

Conclusions: Investigating the 2 subtypes of PRCC on the molecular level, revealed distinct genetic signatures, with different implicated molecular pathways. Our findings highlight the need to segregate between the types in clinical assessment and management; given that current targeted molecular therapies might have different effects on each tumor type. There is also a need to find better identifying markers for pathologists to make the distinction between the subtypes, and accurately reclassify the NOS category.

1034 The MiR-200 Family Has Prognostic Significance in Clear Cell Renal Cell Carcinoma, through Regulation of BAP1, and Related Molecular Pathways

Rola Saleeb, Heba WZ Khella, Qiang Ding, George M Yousef. University of Toronto, Toronto, ON, Canada; Li Ka Shing Knowledge Institute, St. Michael's Hospital, Toronto, ON, Canada.

Background: BAP1 loss of function is associated with established adverse outcome on Clear Cell Renal Cell Carcinoma (ccRCC). miRNAs are small non coding RNAs that regulate gene expression post transcriptionally. The miR-200 family is among the most widely studied miRNAs in Cancer. The current study examines the relationship between the miR-200 family and BAP1 and consequently their influence on ccRCC. Design: Bioinformatic target prediction analysis was performed to identify miRNAs that target $B A P 1$. miR-200 family members miR-200c, miR-200b, and miR-141 were selected for further investigation using qRT-RCR. Analysis was performed on 246 primary ccRCC tumors. Results were validated on the ccRCC Cancer Atlas Genome (TCGA) cohort of 386 patients using Kaplan Meier survival analysis. Gene set enrichment studies (GSEA) were further implemented to classify the possible involved genetic pathways.

Results: Expression of all three members of the miR-200 family was significantly overexpressed in ccRCC cases with tumor progression in relation to the cases with no progression ( $\mathrm{p}=0.0098$ for miR-141 and $\mathrm{p}$ : 0.045 for miR-200c). Survival analysis investigated on TCGA cohort revealed worse survival with increased expression of miR-200b $(H R=1.94, p=0.07)$ and miR-200c $(H R=1.79, p=0.03)$. Results maintained statistical significance on segregating low stage from high stage tumors. GSEA revealed ubiquitin mediated proteolysis pathway involvement integrating both studied genes and miRNAs. The postulated mechanism of action is through post transcriptional inhibition of BAP1 gene and co-involvement in the implicated molecular and epigenetic pathways. Conclusions: miR-200 family members can be used as prognostic markers for ccRCC. The results give further insight into the tumor's pathogenesis and can have clinical value in terms of patients' follow up and targeted molecular therapy.

1035 High Correspondence of HPV Genotypes in Penile Intraepithelial Neoplasia (PeIN) and Invasive Penile Squamous Cell Carcinomas (SCC). A Laser Capture Microdissection (LCM)-PCR Comparative Study of 26 Cases

Diego F Sanchez, Sofia Canete, Nuria Guimera, Maria Jose Fernandez-Nestosa, Elsa F Velazquez, David Jenkins, Wim Quint, Antonio L Cubilla. Facultad de Ciencias Médicas -UNA, Asunción, Paraguay; Instituto de Patología e Investigación, Asunción, Paraguay; DDL Diagnostic Laboratory, Rijswijk, Netherlands; Facultad Politécnica - UNA, San Lorenzo, Paraguay; Miraca Life Sciences and Tuft University, Boston, MA.

Background: About half of penile cancers are HPV-related and the other half are not. Precancerous lesions associated with invasive penile carcinomas are usually of similar histological and cytological features. In HPV positive cases, we do not know the relation of HPV genotype in PeIN and invasive tumors.

Design: The study was designed to compare the morphology of PeIN and corresponding invasive carcinomas a well as their HPV genotypes. There were 26 cases that had in situ and invasive tumors in the same specimen paired as 35 PeIN-Invasive lesions. Invasive cancers were classified in HPV-related (warty, basaloid, medullary) and nonHPV-related (usual, verrucous, papillary NOS, pseudohyperplastic and sarcomatoid). PCR analyses on whole tissue sections (WTS) and laser capture microdissected (LCM) regions were performed by SPF - -DEIA-LiPA ${ }_{25}$ (version 1). DNA quality of negative cases was confirmed by RNAseP/PhHV qPCR.

Results: High agreement (82.86\%, kappa: 0.6591, $\mathrm{P}<0.00005)$ between PeIN and invasive carcinoma morphology was found.

\begin{tabular}{|l|l|}
\hline & PeIN - Invasive paired lesions \\
\hline *W/B morphology agreement (\%) & $16(46)$ \\
\hline Non-HPV-related morphology agreement (\%) & $13(37)$ \\
\hline Disagreement (\%) & $6(17)$ \\
\hline Total & $35(100)$ \\
\hline
\end{tabular}

Eleven patients with 18 paired lesions had HPV positive lesions by LCM-PCR with high concordance $(91.43 \%$, kappa $=0.8287, \mathrm{P}<0.00005)$ of HPV subtype in PeIN and Invasive carcinoma associated.

\begin{tabular}{|l|l|l|}
\hline & PeIN - Invasive paired lesions & HPV genotypes \\
\hline Same HPV genotype (\%) & $16(89)$ & $11,16,18,56$ \\
\hline Different HPV genotype (\%) & $2(11)$ & $11,52,74$ \\
\hline
\end{tabular}

Conclusions: Morphological, architectural and cytological features of PeIN and invasive carcinomas were similar in the majority of the cases. Presence of HPV was associated with warty and basaloid morphology and its absence with keratinizing more differentiated variants of squamous cell carcinoma. In HPV positive cases, viral genotypes were strikingly similar in PeIN and their invasive counterparts indicating a causal relationship.

1036 Clear Cell Papillary Renal Cell Carcinoma Shows Marked Depletion of Mitochondrial Content: A Comparative Differential Diagnostic Study Judy Sarungbam, Ed Reznik, A Ari Hakimi, Ann Bialik, S Joseph Sirintrapun, Hikmat Al Ahmadie, Anuradha Gopalan, Samson W Fine, Ying-Bei Chen, Chris Sander, Victor E Reuter, Satish K Tickoo. MSKCC, NY, NY.

Background: Clear cell papillary renal cell carcinoma (CCPRCC) is a unique variant of RCC in which the HIF pathway is activated through mechanisms distinct from clear cell renal cell carcinoma (ccRCC), including via high levels of intracellular sorbitol. Sorbitol metabolism is known to be closely related to mitochondrial function, including its production of mitochondrial reactive oxygen species. Through detailed investigation of mitochondrial DNA (mtDNA) copy number across tumors from The Cancer Genome Atlas project, we observed that CCPRCC tumors exhibited markedly decreased mitochondrial DNA (mtDNA) levels, compared to the surrounding normal renal parenchyma as well as ccRCC samples. Confirming this fact, whole exome sequencing performed on 4 in-house CCPRCC revealed 10 -fold depletion of mtDNA copy number relative to normal tissue.

Therefore, we investigated the mitochondrial content in CCPRCC by immunohistochemistry using an antibody (MITO Ab2) for the mitochondrial DNAencoded protein MT-CO2, and compared this with the close mimickers, ccRCC with pure clear cell content, and TCEB1-mutated RCC. 
Design: Immunohistochemical stain using the antibody, MITO Ab2 was performed on 14 CCPRCC, 6 ccRCC and 9 TCEB1-mutated RCC. The slides were scanned using the Aperio ScanScope XT systems (Aperio Technologies) at 20X magnification. Immunohistochemical expression was quantified in all digital slides with cytoplasmic algorithm using ImageScope analysis software (version 12; Aperio Technologies, Inc.) and an $\mathrm{H}$-score was calculated.

Results: The results are tabulated.[Table 1]

\begin{tabular}{|c|c|c|c|c|c|}
\hline & Mean & $\begin{array}{l}\text { Standard error } \\
\text { of mean }\end{array}$ & Mean & $\begin{array}{l}\text { Standard error } \\
\text { of mean }\end{array}$ & P value \\
\hline & \multicolumn{2}{|c|}{ CCPRCC } & \multicolumn{2}{|c|}{ ccRCC } & \\
\hline \multirow[t]{2}{*}{ Cytoplasm: H-score } & 0.05 & 0.03 & 17.66 & 9.78 & 0.010 \\
\hline & \multicolumn{2}{|c|}{ CCPRCC } & \multicolumn{2}{|c|}{ TCEB 1-mutated RCC } & \\
\hline \multirow[t]{2}{*}{ Cytoplasm: H-score } & 0.05 & 0.03 & 15.50 & 6.35 & 0.006 \\
\hline & \multicolumn{2}{|c|}{ ccRCC } & \multicolumn{2}{|c|}{ TCEB1-mutated RCC } & \\
\hline Cytoplasm: H-score & 17.66 & 9.78 & 15.50 & 6.35 & 0.849 \\
\hline
\end{tabular}

Conclusions: 1 . Clear cell papillary RCC shows marked depletion of mitochondrial content compared to both TCEB1-mutated RCC and clear cell RCC.

2. Immunohistochemistry for MITO Ab2 is a useful way of differentiating clear cell papillary RCC from close mimickers, TCEB 1-mutated RCC and clear cell RCC.

3. The relationship of high sorbitol content with marked paucity of mitochondria in clear cell papillary RCC requires further investigation.

1037 The Relationship among ERG, PTEN and SPINK1 in Transition and Peripheral Zone Prostate Cancers in Japanese Patients

Shun Sato, Hiroyuki Takahashi, Takashi Yorozu, Takahiro Kimura, Masahiro Ikegami. Jikei University School of Medicine, Tokyo, Japan.

Background: We have previously reported locational predominance on transition zone (TZ) in Japanese prostate cancer, and revealed that ERG overexpression was less frequent in TZ cancer than in peripheral zone (PZ) cancer. In the past, SPINK1 overexpression and PTEN loss have been shown to be central roles in prostate carcinogenesis and progression. In this aspect, we conducted further immunohistochemical analysis based on the zonal difference focusing on ERG, PTEN and SPINK1.

Design: A total of 211 radical prostatectomy specimens were utilized. Immunohistochemistry of PTEN and SPINK1 were performed in all cases. Status of PTEN loss and SPINK1 positivity were evaluated; the relationship between ERG overexpression and SPINK1 positivity was examined, in all cases, TZ cancers, and $\mathrm{PZ}$ cancers, respectively.

Results: The loss of PTEN was significantly more frequent in PZ cancers than TZ ones $(\mathrm{p}=0.0181$ ). No significant difference was shown in SPINK1 positivity between $\mathrm{TZ}$ and $\mathrm{PZ}$ cancers $(\mathrm{p}=0.1773)$.

\begin{tabular}{|l|l|l|l|}
\hline & TZ $(88$ cases $)$ & PZ $(122$ cases $)$ & p-value \\
\hline ERG overexpression (case/\%) & $7 / 8.0$ & $26 / 21.3$ & 0.0117 \\
\hline PTEN loss (case/\%) & $5 / 5.7$ & $21 / 17.2$ & 0.0181 \\
\hline SPINK1 positive (case/\%) & $17 / 19.3$ & $15 / 12.3$ & 0.1773 \\
\hline
\end{tabular}

With regards to the relationship between ERG and SPINK 1, 100\% of ERG positive tumors showed negative for SPINK 1 in all cases $/ \mathrm{PZ}$ cancers $(\mathrm{p}=0.0061,0.039)$. In ERG negative cancers, 18 and $15.6 \%$ showed positive for SPINK1 in all cases and PZ cancers, respectively. Similar tendency was shown in TZ cancers.

\begin{tabular}{|c|c|c|c|}
\hline & ERG positive & negative & $\mathrm{p}$-value \\
\hline \multicolumn{4}{|l|}{ all cases } \\
\hline SPINK1 positive (\%) & $0(0)$ & $32(18.0)$ & \multirow{2}{*}{0.0061} \\
\hline negative (\%) & $33(100)$ & $146(82.0)$ & \\
\hline \multicolumn{4}{|l|}{ TZ cases } \\
\hline SPINK1 positive (\%) & $0(0)$ & $17(21.0)$ & \multirow{2}{*}{0.3376} \\
\hline negative (\%) & $7(100)$ & $64(79.0)$ & \\
\hline \multicolumn{4}{|l|}{ PZ cases } \\
\hline SPINK1 positive (\%) & $0(0)$ & $15(15.6)$ & \multirow{2}{*}{0.0390} \\
\hline negative (\%) & $26(100)$ & $81(84.4)$ & \\
\hline
\end{tabular}

Conclusions: The differences of the frequency of ERG overexpression and PTEN loss between $\mathrm{TZ}$ and $\mathrm{PZ}$ cancers suggest presence of different molecular pathways between them, especially, ERG overexpression and PTEN loss are not frequent phenomena in TZ cancers, implying the presence of other factors in cancer development and progression. The alternative role of ERG and SPINK1 is suggested.

1038 Orchidectomies for Testicular Neoplasms Less Than $1 \mathrm{~cm}$ in Diameter: Victims of Modern Imaging Technology?

Glenda Scandura, Wendy Ansell, Jonathan Shamash, Daniel Berney. Queen Mary University of London, London, Greater London, United Kingdom; Barts Health NHS Trust, London, United Kingdom.

Background: The acronym 'VOMIT' stands for 'Victims Of Modern Imaging Technology'. The ability of radiological ultrasound to detect small and impalpable testicular lesions has lead to a potential increase in patients with partial or total orchidectomies for benign lesions. We wished to investigate the pathology of small testicular lesions which were unlikely to be detected on clinical grounds alone.
Design: From Jan 2003 to July 2015, the pathological reports of 1,544 patients from Bartshealth NHS Trust including consult cases were reviewed and cases with a maximum diameter of $<1 \mathrm{~cm}$ were selected. Clinical records were also accessed, where available, to look for patient demographics and pre-diagnostic levels of serum markers.

Results: 59 cases $(3.8 \%)$ with a tumor size $<1 \mathrm{~cm}$ were identified and of these 12 cases had a diameter $<5 \mathrm{~mm}$. 43 cases ( $75 \%$ ) were benign. 11 of the 12 cases $<5 \mathrm{~mm}$ underwent orchidectomy. Tumor markers were available on 57 of the cases and all showed a normal LDH, AFP and B-hCG. All the malignant cases were germ cell tumors: 10 seminomas $(62.5 \%)$ and 6 non-seminomas (37.5\%). Only 1 germ cell tumor had a diameter of $<5$ $\mathrm{mm}$ and this was a regressed tumor within an $18 \mathrm{~mm}$ area of granulomatous inflammation. The 'benign' cases included 21 sex cord stromal tumors including 12 Leydig cell tumors $(28 \%), 7$ Sertoli cell tumors $(16.4 \%)$ and two mixed sex cord stromal tumors. None showed any malignant features. The remaining 22 lesions (37\%) included: 1 Sertoli cell nodule, 2 Leydig cell hyperplasias, 2 tunica albuginea cysts, 2 haemangiomas, 1 necrotic nodule, 2 epidermoid cysts, 2 epididymal cysts, 3 pseudotumors, 1 ectopic nodule of adrenal cortex, 1 non-specific chronic inflammation, 1 cystic transformation of rete testis, 2 adenomatoid tumours and 2 fibromas of the tunica albiginea.

Conclusions: Small testicular lesions, identified by radiology, are unlikely to be malignant, especially if $<5 \mathrm{~mm}$ where no malignant cases were detected. Tumor markers appear unhelpful in the distinction of these small tumors. We suggest that regular ultrasound surveillance ought to be more widely used for testicular lesions of this size. Testicular tumours now have a very high cure rate and changes in size of lesions may be monitored prospectively with minimal risk of incresed morbidity. We suggest that patients who undergo an orchidectomy for lesions $<5 \mathrm{~mm}$ are 'Victims of Modern Imaging Technology'.

\section{Spectrum of Phosphorylated STAT3 (pSTAT3) Expression in a} Large Cohort of Common Renal Tumors

Benjamin Scheier, Aaron Udager, Tina Fields, Dafydd Thomas, Thomas J Giordano, $J$ Stuart Wolf, Bruce Redman, Lakshmi P Kunju. University of Michigan Health System, Ann Arbor, MI.

Background: STAT3 is a signaling-regulated transcription factor that affects cellular proliferation and apoptosis. Cytoplasmic STAT3 is phosphorylated in response to cytokines (i.e., IL-6) and growth factors (i.e., VEGF), and pSTAT3 translocates to the nucleus to regulate gene expression. High pSTAT3 expression has been reported to be a poor prognostic factor in renal cell carcinoma (RCC), and therefore, we sought to examine pSTAT3 expression by immunohistochemistry (IHC) in a large cohort of common renal tumors.

Design: pSTAT3 IHC was performed using a primary rabbit monoclonal antibody (D3A7, Cell Signaling Technology, Inc.), which recognizes phosphorylated Tyr705 of STAT3, on three tissue microarrays (TMAs) containing 287 unique tumor specimens (at least two cores per specimen). A semi-quantitative score was calculated as the average product of tumor cell staining intensity $(0-3)$ and percentage of cells positive $(0-100)$ (overall product score range $=0-300$ )

Results: At least one evaluable core was available for 268 (93.4\%) specimens (Table 1). Clear cell RCC (CCRCC) showed significantly higher average pSTAT3 staining compared to all other tumor types $(\mathrm{P}<0.001)$; there were no other significant differences in $\mathrm{pSTAT} 3$ staining between tumor types $(\mathrm{P}>0.05)$. When $\mathrm{CCRCC}$ were stratified as high or low pSTAT3 expression based on the median average product score, tumors with high pSTAT3 staining were significantly more likely to be low nuclear grade $(\mathrm{P}<0.05)$; there was no significant association between pSTAT3 staining and pathologic stage $(\mathrm{P}>0.05)$.

\begin{tabular}{|l|l|l|l|l|l|}
\hline Tumor Type & N & MEAN & MEDIAN & MIN & MAX \\
\hline All tumors & 268 & 26.2 & 7.5 & 0 & 270 \\
\hline Clear cell RCC & 178 & 35.9 & 12.9 & 0 & 270 \\
\hline Papillary RCC & 37 & 6.3 & 0 & 0 & 55 \\
\hline Chromophobe RCC & 19 & 4.1 & 0 & 0 & 70 \\
\hline Oncocytoma & 20 & 13.8 & 5.0 & 0 & 70 \\
\hline Urothelial carcinoma & 14 & 2.9 & 0 & 0 & 30 \\
\hline
\end{tabular}

Conclusions: CCRCC demonstrates variable pSTAT3 expression, including a subset of tumors with very high pSTAT3 staining. Other common renal tumors do not show significant pSTAT3 expression. Interestingly, CCRCC with high pSTAT3 expression tend to be lower nuclear grade. Given the previously reported association between high pSTAT3 expression and poor outcome, these data suggest that pSTAT3 IHC may be a helpful biomarker for risk stratifying patients with low grade CCRCC.

1040 Radical Prostatectomy Outcomes of Prostatic Adenocarcinoma Diagnosed after Initial Diagnosis of Atypical Glands on Prostate Needle Biopsy

Alpa Shah, Ramya Gadde, Javier Arias-Stella, Sean R Williamson, Nilesh S Gupta. Henry Ford Hospital, Detroit, MI

Background: Few studies have reviewed radical prostatectomy (RP) outcomes of prostate adenocarcinoma ( $\mathrm{PCa}$ ) detected on repeat biopsy after initial diagnosis of atypical glands (ATYP). The aim of this study is to review the location, tumor volume, grade, stage, margin and lymph node status of these tumors based on review of PNB and RP parameters.

Design: Between 2009-2013, all cases of first-time diagnosis of ATYP on PNB were reviewed. Patients with previous or synchronous biopsy diagnosis of $\mathrm{PCa}$ and patients without follow-up biopsy data were excluded. Presence or absence of HGPIN and location of ATYP and HGPIN were noted. Number of positive cores, location, grade, and percentage of PCa was noted on all PNB. All staging and grading parameters of the tumor were recorded on RP. 
Results: 2471 PNBs were performed during the study period. 283 of these were diagnosed as ATYP. After applying exclusion criteria our final study population consisted of 128 patients with a first-time diagnosis of ATYP.

42 (33\%) of these patients developed PCa on repeat biopsy. Vast majority 37/42 (88\%) of post-ATYP PCa were within favorable prognostic groups of I and II (see Table 1). Few cases fell in Group III/GS $7(3+4)-4(10 \%)$, and a rare case in Group V/10 $(5+5)-1(2 \%)$.

\begin{tabular}{|l|l|l|l|l|l|}
\hline $\begin{array}{l}\text { Prognostic Group } \\
\text { [Gleason Grade] }\end{array}$ & $\begin{array}{l}\text { I } \\
{[\mathbf{6}(\mathbf{3}+\mathbf{3})]}\end{array}$ & $\begin{array}{l}\text { II } \\
{[\mathbf{7 ( 3 + 4 )}]}\end{array}$ & $\begin{array}{l}\text { III } \\
{[\mathbf{7 ( 4 + 3 ) ]}}\end{array}$ & IV [8(4+4)] & $\begin{array}{l}\text { V [9(4+5), } \\
\mathbf{1 0 ( 5 + 5 ) ]}\end{array}$ \\
\hline Number of Cases (PNB) & 33 & 4 & 4 & 0 & 1 \\
\hline
\end{tabular}

36 cases $(85.7 \%)$ of $\mathrm{PCa}$ on repeat PNB were present at a contiguous location to a previous ATYP diagnosis. Six cases (14.3\%) showed PCa in discontiguous location. 14/42 PCa diagnosed on PNB underwent RP at our institution. RP parameters are listed in Table 2.

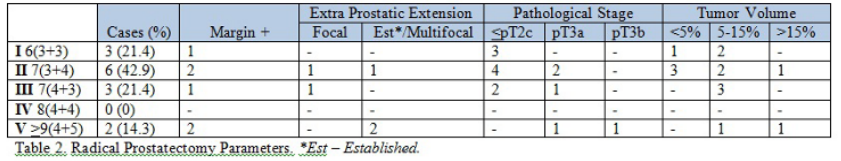

Conclusions: PCa after initial ATYP diagnosis are low Gleason grade (Prognostic groups I and II) with excellent RP outcomes. It is rare to find low volume high grade PCa within the ATYP group and may be related to sampling on initial PNB. Location of ATYP highly correlates with the final location of PCa both on repeat PNB and RP.

1041 Diagnosis of Limited Prostate Adenocarcinoma on Core Needle Biopsy: An Improvement of Interobserver Reproducibility Using Comprehensive Morphological and Molecular Criteria

Rajal Shah, Gioacchino Leandro, James Bentley, Monica Chaterjee, Gloria Romerocases, Wei Tian, Yousef Tadros, Jiyoon Yoon, Savvas Mendrinos. Miraca Life Sciences, Irving, TX; IRCCS De Bellis, Castellana Grotte, Italy.

Background: Considerable difficulties persist amongst pathologists in classifying "Atypical glands suspicious for adenocarcinoma" (ATYP) vs. limited prostate adenocarcinoma (PCa) on core needle biopsy (NBX). Reducing diagnostic variability associated with this diagnosis is critical to patient management. The aims of the study were: 1) to generate a comprehensive list of major and minor features to diagnose limited PCa; 2) to test interobserver reproducibility of ATYP classification using comprehensive criteria.

Design: 71 selected NBXs with the diagnosis of ATYP including available immunohistochemical stains were reviewed by 8 study participants. Participants were blinded to original diagnosis and were asked to classify ATYP lesion as benign, atypical or PCa based on their criteria. A final comprehensive list of 13 major and 11 minor criteria to diagnose limited PCa was generated based on literature review and consensus criteria utilized by participants. Participants were finally asked to reclassify the same set of 71 ATYP cases based on comprehensive criteria. Interobserver reproducibility of overall diagnostic agreement ( $75 \%$ agreement) was assessed.

Results: In addition to conventional criteria, noticeable major criteria for classification of limited PCa in the final list included: 1) significant nuclear enlargement with hyperchromasia in absence of prominent nucleoli; 2) require more glands or a component of conventional acinar PCa when dealing with pseudohyperplastic, PIN-like or atrophic features; 3 ) focus of concern not only present at the edge of the core; 4) lack of intermingling high grade prostatic intraepithelial neoplasia (HGPIN) and atrophy; 5) ERG positivity in small atypical glands where HGPIN is excluded; 6) PTEN loss and/or ERG overexpression in atypical intraductal proliferation suggest intraductal carcinoma over HGPIN. Interobserver reproducibility measured by Fleiss' kappa for initial and subsequent classification using comprehensive major criteria was 0.36 and 0.59 respectively $(\mathrm{p}=0.006)$. Application of above 6 major criteria in addition to conventional criteria showed significant improvement for $100 \%$ concordance $(p=0.011)$ and reduction for no consensus $(\mathrm{p}=0.0004)$ categories compared to initial classification. Conclusions: By using contemporary approach to classification of ATYP, a higher level of agreement was achieved by a group of pathologists with varying level of training and experience in Urologic pathology.

1042 High Grade Prostatic Intraepithelial Neoplasia with Adjacent Small Atypical Glands (PINATYP) on Core Needle Biopsy: A Clinicopathological Analysis of $\mathbf{5 4}$ Cases with Emphasis on Predictors of Prostate Cancer Rajal Shah, Wei Tian, Ming Zhou, Jianbo Li. Miraca Life Sciences, Irving, TX; New York University Langone Medical Center, New York, NY; Cleveland Foundation Clinic, Cleveland, $\mathrm{OH}$

Background: When high-grade prostatic intraepithelial neoplasia (HGPIN) with adjacent small atypical glands (PINATYP) is encountered in prostate needle biopsies, the differential diagnosis includes tangential sectioning or outpouching of HGPIN glands versus microinvasive adenocarcinoma ( $\mathrm{PCa}$ ) arising from HGPIN. This distinction is challenging due to lack of well-defined features of micro invasion. Objective of the study was to identify risk factors for subsequent PCa detection for men with PINATYP. Design: We analyzed clinicopathological variables of 54 PINATYP lesions who had at least one follow up biopsy. Immunohistochemistry for PIN4 (basal cell markers p63, high molecular weight cytokeratin CK5/14 and racemase) was available in all cases. By definition, all cases had lack of basal cells staining in at least some atypical small glands adjacent to HGPIN.

Results: A mean of 1.09 rebiopsies (range, 1 to 2 ) were performed with a mean followup time of 5.7 months (range, 1 to 30). Five patients had 2 follow up biopsies. Overall
$\mathrm{PCa}$ detection rate on follow up biopsies was $41 \%$. The average age at presentation was 64 years (range, 48 to 82 ); mean PSA was $6.9 \mathrm{ng} / \mathrm{ml}$ (range, 0.6 to 76 ). In $83 \%$ of cases HGPIN had tufting pattern, and remaining cases showed a mixture of other patterns (flat, micropapillary and cribriform). The following histologic or immunohistochemical features did not predict PCa on repeat biopsy: ratio of small glands to HGPIN glands; the greatest distance of small glands from HGPIN; more than one core involved by HGPIN; the presence of macronucleoli, crystalloids or blue mucin; architecture of small atypical glands (random, crowded vs. infiltrative); patchy vs. complete loss of basal cell staining in small atypical glands; architecture of small glands without basal cells staining (random, crowded vs. infiltrative); and racemase expression. Majority (82\%) of $\mathrm{PCa}$ detected in subsequent biopsy were low Gleason score $3+3=6$; remaining being Gleason score $3+4(9 \%)$ and $4+3(9 \%)$.

Conclusions: PINATYP has significantly higher risk of PCa detection than HGPIN alone. In preliminary observations on a small cohort, no additional clinicopathological variables are reliably useful in predicting which men with this lesion will have PCa on rebiopsy; therefore, all men with PINATYP need to undergo repeat biopsy.

1043 Extensive Retraction Clefts in Invasive Urothelial Carcinoma Are Associated with Aggressive Tumor Behavior

Tanmay Shah, Matthew G Kaag, Jay D Raman, Wilson Chen, Truc Tran, Sudhir Kunchala, David J DeGraff, Guoli Chen, Joshua I Warrick. Penn State Hershey Medical Center, Hershey, PA.

Background: Micropapillary morphology, seen as small tumor nests with surrounding retraction artifact, is associated with aggressive clinical behavior in several cancer types, including urothelial and breast. It has recently been shown that extensive retraction clefts in invasive breast cancer are also associated with aggressive clinical behavior, independent of micropapillary histology. We thus set out to determine if the same association between retraction artifact and clinical outcome is present in urothelial carcinoma.

Design: A series of radical cystectomy specimens from patients with invasive bladder cancer was assembled. Cases of primary adenocarcinoma and urothelial carcinoma with any component of high risk morphology (plasmacytoid, micropapillary, nested, sarcomatoid, small cell) were excluded. Cases for which lymph node sampling was not available $(\mathrm{pNx})$ were also excluded. 117 cases remained after exclusions. Tumor histology for all cases was methodically re-reviewed, with documentation of the presence of extensive retraction clefts in invasive urothelial carcinoma, defined as extensive retraction artifact surrounding invasive nests of carcinoma. Pathologic staging parameters (all 117 cases) and clinical outcomes were documented (extended outcomes limited to 88 patients). Associations among retractions clefts, staging, and clinical variables were determined.

Results: Of the 117 cases of invasive carcinoma, 21 (18\%) had extensive retraction clefts. The presence of retraction clefts in invasive carcinoma was associated with lymph node metastasis $(57 \% \geq \mathrm{pN} 1$ with retraction clefts $\underline{\text { vs }} 27 \% \geq \mathrm{pN} 1$ without retraction clefts; $\mathrm{p}<0.01$ ). Death from bladder cancer was more common in cases with retraction clefts, though this association did not achieve statistical significance $(\mathrm{OR}=3.0(0.5-21)$, $\mathrm{p}=0.23)$. The same was found for metastatic disease $(\mathrm{OR}=2.8(0.9-8.6), \mathrm{p}=0.11)$ and recurrence as metastatic disease $(\mathrm{OR}=2.3(0.81-6.7), \mathrm{p}=0.16)$. Positive associations were not observed for pathologic tumor stage, overall survival, or any recurrence.

Conclusions: Conventional urothelial carcinoma with extensive retraction clefts is significantly associated with the presence of lymph node metastases, and may be indicative of more aggressive disease.

1044 Sarcomatoid Clear Cell RCC Shows a Distinct Molecular Pathogenesis and Driver Mutation Profile

Kanishka Sircar, Zixing Wang, Tae-Beom Kim, Bo Peng, Jose A Karam, Chad Creighton, Chi-Wan Chow, Jaime Rodriguez Canales, Pheroze Tamboli, Gordon Mills, Kenna Shaw, Ken Chen. MD Anderson, Houston, TX; Baylor College of Medicine, Houston, TX. Background: Sarcomatoid clear cell RCC (SccRCC) represents the most aggressive and treatment resistant clinicopathologic phenotype of clear cell RCC. The paucity of high quality genome wide molecular examination of SccRCC has hindered our understanding of this entity. The aim of this study was to interrogate the mutational, copy number and transcriptomic landscape of SccRCC and to compare these data with non-sarcomatoid clear cell RCC (ccRCC).

Design: We evaluated $59 \mathrm{SccRCC}$ and $465 \mathrm{ccRCC}$ samples from two patient cohorts: local SccRCC cases ( $n=28$ samples); and TCGA renal carcinoma cases ( $n=31$ SccRCC samples and $\mathrm{n}=465$ ccRCC samples ( $\mathrm{G} 1, \mathrm{n}=10 ; \mathrm{G} 2, \mathrm{n}=214 ; \mathrm{G} 3, \mathrm{n}=191 ; \mathrm{G} 4, \mathrm{n}=50)$ ). We performed whole exome sequencing (WEX, Illumina Hi-Seq) and RNA-seq (NimBlegen and Illumina HiSeq) on local SccRCC cases. DNA mutations and copy number were assessed using WEX data and mRNA expression was assessed using RNA-seq data run through in-house developed pipelines. TCGA data was analyzed based on the Broad Institute fire-hose pipeline.

Results: SccRCC showed a lower rate of two-hit loss (concurrent mutation and copy loss) for the 3p genes VHL and PBRM1 compared to ccRCC, with significance achieved compared to all grades, except grade 4 ccRCC: $\mathrm{P}=0.016$, vs $\mathrm{G} 1 ; \mathrm{P}=0.0094$, vs $\mathrm{G} 2$; $\mathrm{P}=0.0036$, vs G3; $\mathrm{P}=0.209$, vs G4. Mutations in PTEN $(\mathrm{P}=0.028)$, TP53 $(\mathrm{P}=0.0004)$ and $\operatorname{RELN}(\mathrm{P}=0.0048)$ were individually associated with SccRCC and were usually mutually exclusive. A mutation in any one of these 3 genes was associated with SccRCC compared to all ccRCC ( $\mathrm{P}=1.09 \mathrm{E}-06)$, including grade 4 ccRCC $(\mathrm{P}=0.029)$. Focal copy loss of PTEN was enriched in SccRCC with concordant upregulation of PD-L1 mRNA expression ( $\mathrm{P}=0.0038$ ). Ingenuity pathway analysis of transcript data comparing SccRCC to ccRCC of advanced stage (III/IV) showed alteration of the VEGF (activated, $\mathrm{P}=1.8 \mathrm{E}-20$ ), TGFb1 (activated, $\mathrm{P}=2.7 \mathrm{E}-20$ ), TP53 (inhibited, $\mathrm{P}=1.8 \mathrm{E}-17$ ) and $\mathrm{mTOR}$ pathways (activated, $\mathrm{P}=1.8 \mathrm{E}-03$ ) in SccRCC. 
Conclusions: Sarcomatoid ccRCC shows a different early molecular pathogenesis in terms of reduced two-hit loss of the $3 p$ tumor suppressor genes $(V H L, P B R M 1)$ and enrichment of different driver mutations (PTEN, TP53, RELN). Taken together with transcriptome analysis, these data suggest the potential for pathway based (TGFb1, mTOR) therapies and checkpoint blockade (anti- PD-L1) immunotherapy in the treatment of SccRCC.

1045 Prostate Cancer (PCa) with Overlapping Features of Small Cell Carcinoma and Acinar Adenocarcinoma: A Critical Appraisal of Morphology and Correlation with Immunohistochemical (IHC) Markers Deepika Sirohi, Steven Smith, Chisato Ohe, Mariza de Peralta-Venturina, Daniel Luthringer, Scott A Tomlins, Moushumi Suryavanshi, Arkadiusz Gertych, Beatrice Knudsen, Mahul B Amin. Cedars-Sinai Medical Center, Los Angeles, CA; Virginia Commonwealth University Health System, Richmond, VA; University of Michigan Medical School, Ann Arbor, MI; Rajiv Gandhi Cancer Institute and Research Center, New Delhi, India.

Background: A consensus classification for PCa with neuroendocrine differentiation (NED) has been recently proposed. A provisional category was named $P C a$ with overlapping features of Sm CC and acinar PCa ("overlap PCa") and includes PCa with NED in patients with or without potent androgen blockade. Recently, loss of Cyclin D1 (CCND1) and expression of thyroid transcription factor-1 (TTF1) have been observed in SmCC. Therefore we evaluate the expression of these markers in overlap PCa and compare their expression to the other two established categories of PCa with NED. Design: We analyzed 43 areas in 35 cases of overlap PCa $(n=15), \operatorname{SmCC~}(n=11)$ and acinar PCa with IHC evidence of NED ( $\mathrm{n}=17$ ) using markers of NED (synaptophysin, chromogranin, CD56), prostatic histogenesis (PSA, PSMA, NKX3.1, p501S), AR, Ki-67, CCND1 (SP4 clone) and TTF1 (SP141 clone).

Results: Overlap PCas show hybrid morphologic features of SmCC and conventional $\mathrm{PCa}$, including areas with abundant cytoplasm $(100 \%)$, focal acinar differentiation $(60 \%)$, variable nucleolar prominence $(100 \%)$, lack of nuclear molding $(67 \%)$, salt and pepper chromatin (87\%), brisk mitoses $(67 \%)$ and sheet-like growth $(40 \%)$. Overlap PCas also demonstrate a hybrid immunophenotype of prostate histogenesis, AR expression and cell proliferation. In addition, CCND1 and TTF1 were expressed in $50 \%$ and $8 \%$ of overlap PCa cases, respectively.

\begin{tabular}{|l|l|l|l|l|l|}
\hline & AR (\%) & $\begin{array}{l}\text { Expression of at least } \\
\text { 1 Prostate histogenesis } \\
\text { marker (\%) }\end{array}$ & $\begin{array}{l}\text { Cyclin D1 } \\
(\%)\end{array}$ & TTF-1 (\%) & $\begin{array}{l}\text { Ki67>50\% } \\
(\%)\end{array}$ \\
\hline $\begin{array}{l}\text { Acinar with } \\
\text { NED }\end{array}$ & 100 & 100 & 86 & 7 & 0 \\
\hline Overlap PCa & 58 & 87 & 50 & 8 & 58 \\
\hline SmCC & 0 & 0 & 9 & 67 & 90 \\
\hline
\end{tabular}

Conclusions: Taken with morphology and markers of NED, loss of CCND1 (signaling defect of Rb function) may be of help in identification of PCas with overlapping features between SmCC and acinar PCas. This immunoprofile may be used prospectively to identify an actionable (including drugs in the $\mathrm{Rb}$ axis) subset of high grade $\mathrm{PCa}$ with NED without all diagnostic features of SmCC but sharing the aggressive, highly proliferative phenotype. In contrast to SmCC, overlap PCas express TTF1 only infrequently, at a frequency similar to conventional PCa with NED. Thus TTF1 expression favors an explicit diagnosis of SmCC.

1046 Clinical and Immunohistochemical (IHC) Spectrum of Renal Cell Carcinomas (RCCs) with Aberrant Fumarate Hydratase (FH)-Related Studies: Report from the High-Grade Distal Nephron Adenocarcinoma (HDNA) International Consortium

Deepika Sirohi. Cedars-Sinai Medical Center, Los Angeles, CA.

Background: An emerging subset of HDNAs putatively arise in the setting of hereditary leiomyomatosis \& RCC syndrome (HLRCC-RCCs). These show lack of FH \& 2 SC expression by IHC, though more data are needed to understand the clinicopathologic spectrum of cases with aberrancy of these markers.

Design: 115 cases of HDNA were collected from 25 institutions \& 10 countries (HDNA International Consortium). After clinicopathologic \& IHC correlation (INI1, OCT4, FH \& $2 \mathrm{SC}$ ), a subset were identified by their harboring morphologic features compatible with that described for HLRCC-RCCs, with aberrant FH \&/or 2SC staining.

Results: A total of 33 cases comprising of 3 groups were identified: 1) "Consistent with HLRCC" (n=7, FH-/2SC+, syndromic history/stigmata); 2) "FH-deficient RCC" $(\mathrm{n}=17, \mathrm{FH}-/ 2 \mathrm{SC}+$ cases without syndromic history/stigmata); and 3$)$ " $\mathrm{FH}$-suspicious $\mathrm{RCC}$ " ( $\mathrm{n}=9, \mathrm{FH}+/$ - heterogeneous but $2 \mathrm{SC}+$ cases). The characteristics of these groups are presented.

\begin{tabular}{|l|l|l|l|}
\hline Clinicopathologic Features & $\begin{array}{l}\text { Consistent with } \\
\text { HLRCC (N=7) }\end{array}$ & $\begin{array}{l}\text { FH-Deficient } \\
\text { RCC (N=17) }\end{array}$ & $\begin{array}{l}\text { FH-suspicious } \\
\text { RCC (N=9) }\end{array}$ \\
\hline Mean Age (range) & $34.4(21-48)$ & $46.7(20-67)$ & $45.0(16-71)$ \\
\hline Sex - M:F & $1.3: 1$ & $4.7: 1$ & $2: 1$ \\
\hline Family history (\%) & 100 & 0 & 0 \\
\hline Stigmata / syndrome (\%) & 100 & 0 & 0 \\
\hline Tumor size (cm) (range) & $6.6(3-14)$ & $9.2(4.1-18)$ & $9.9(3.3-12)$ \\
\hline Advanced pT stage (3, 4) (\%) & 57 & 88 & 67 \\
\hline Lymph node metastasis (\%) & 60 & 59 & 57 \\
\hline Systemic metastasis (\%) & 29 & 81 & 50 \\
\hline $\begin{array}{l}\text { Dead of disease (\%) (follow-up } \\
\text { duration) }\end{array}$ & $20(64$ month) & $80(15$ month) & 67 (17 month) \\
\hline Infiltrating glands (\%) & 29 & 47 & 44 \\
\hline Tubular, papillary (\%) & 71 & 88 & 67 \\
\hline Cords, nests, solid (\%) & 86 & 76 & 67 \\
\hline Intracystic papillary (\%) & 57 & 53 & 44 \\
\hline Sieve-like, cribriform (\%) & 43 & 59 & 89 \\
\hline Tubulocystic (\%) & 29 & 71 & 78 \\
\hline $\begin{array}{l}\text { CMV-like nucleoli (+, focal; } 2+, \\
\text { non-focal) (\%) }\end{array}$ & $100(14,86)$ & $100(23,77)$ & $88(44,44)$ \\
\hline Cons
\end{tabular}

Conclusions: Contemporary workup of FH-deficient RCC breaks down into 3 groups sharing aggressive clinical \& histologic features. Syndromic stigmata \& history remain conspicuously absent in FH-deficient \& FH-heterogeneous groups, even when specifically elicited retrospectively. Until further clarity emerges, we propose the term FH-deficient RCC as a unifying provisional term for these cases, reserving the hereditary designation as a clinical designation after genetic studies and counseling.

1047 Clinico-Pathologic Analysis of 12 Cases of Primary Renal Glomus Family of Tumors

Deepika Sirohi, Jonathan I Epstein, Bonnie L Balzer, Oleksandr N Kryvenko, Swetha Paluru, Mariza de Peralta-Venturina, Mahul B Amin. Cedars-Sinai Medical Center, Los Angeles, CA; The Johns Hopkins Hospital, Baltimore, MD; University of Miami Miller School of Medicine, Miami, FL.

Background: Renal glomus tumors (GT) are exceptionally rare with 13 reported cases ( 1 clinically malignant; largest series of 3 cases). The differential includes various renal epithelial tumors, juxtaglomerular cell tumor (JGCT) \& epithelioid angiomyolipoma. Design: 12 cases were identified from 3 institutions.

Results: The age ranged from 17 to 76 yrs. (mean-50), with a M:F ratio of 1.4:1. Size ranged from 1.7 to $7.5 \mathrm{~cm}$ (mean-3.8 cm). History of hypertension (available in 8 patients) was positive in $6(75 \%)$ which persisted even after surgical resection, including in 2 younger patients ( $17 \& 30$ yrs). Radiologically tumors mimicked renal cell carcinoma (RCC). The diagnoses included: GT (7); glomangiomyoma (1); perivascular myoid family of tumors with overlapping features of glomus \& smooth muscle neoplasms (2); perivascular myoid tumor with atypical features (1); and GT with atypical features (1). The histology was remarkably typical resembling that of the soft tissue counterparts. Morphology ranged from that of classic glomus tumors those with prominent vascularity to others showing combined solid compact growth \& inconspicuous vasculature. Some areas of the tumor resembled clear cell RCC. Symplastic changes were seen in two cases \& atypical features were seen in 2 cases [increased cellularity (2/2), mitotic activity (2/2) - (10 per 10 high power fields in 1 case $\&$ rare in another, necrosis $(1 / 2)$, moderate cellular atypia $(1 / 2) \&$ perinephric extension $(1 / 2)]$. Immunohistochemical (IHC) results were as follows: smooth muscle actin $(91 \%)$, collagen4- $(100 \%)$, CD34-(55\%), calponin $(100 \%)$, desmin $(25 \%)$. Follow up ( 7 cases $)$ ranging from 1- 62 months (mean 13.8) including one case with atypical features. All cases including those over $2 \mathrm{~cm}$ showed no evidence of progression.

Conclusions: Because morphology and IHC of renal GT overlap considerably with JGCT, reliance on identification of characteristic morphologic features analogous to somatic soft tissue GTs, and correlation with serum renin levels and a clinical history of severe hypertension resolving after tumor resection, are important to distinguish renal GT from JGCT. Clear cell RCC may be mimicked, particularly in scant biopsy specimens. Clinically malignant behavior is rare in renal GT and is not tightly correlated to predictors of aggressive behavior in GT in extrarenal sites. Therefore, clinical parameters including deep location and size $>2 \mathrm{~cm}$ need to be modified for tumors occurring in the kidney.

1048 A Novel Low Grade Morphologic Variant of Hereditary Leiomyomatosis-Renal Cell Carcinoma Syndrome-Associated Renal Cell Carcinoma (HLRCC-RCC)?

Steven Smith, Deepika Sirohi, Chisato Ohe, Jonathan McHugh, Jason Hornick, Jigna Kalariya, Sushil Karia, Katie Snape, Shirley Hodgson, Scott Tomlins, Rohit Mehra, Mahul B Amin. Virginia Commonwealth University Health System, Richmond, VA; Cedars-Sinai Medical Center, Los Angeles, CA; University of Michigan, Ann Arbor, MI; Brigham and Women's Hospital, Boston, MA; B T Savani Kidney Hospital, Rajkot, India; St Georges Hospital, London, United Kingdom.

Background: HLRCC-RCC represents an emerging class of high grade tubulopapillary $\mathrm{RCC}$, arising in the setting of familial cutaneous and uterine leiomyomatosis with germline fumarate hydratase $(\mathrm{FH})$ mutation. In contrast, succinate dehydrogenase subunit B (SDHB)-deficient RCC presents in the setting of hereditary paragangliomas and gastrointestinal stromal tumors, showing a distinctive low grade oncocytic 
morphology, cytoplasmic flocculence/vacuolation, and inclusions. These tumors share dysfunction in successive Krebs cycle enzymes with related oncometabolic consequences.

Design: We report three cases, in confirmed HLRCC, where the RCC morphology observed is a low grade oncocytic carcinoma, morphologically reminiscent of SDHBdeficient RCC

Results: Nephrectomies from three male patients, 11, 35, and 40 years, showed $6 \mathrm{~cm}$, multinodular up to $9 \mathrm{~cm}$, and paired $0.4 \mathrm{~cm}$ and $<0.1 \mathrm{~cm}$ tan yellow neoplasms, respectively. In the third, a separate, synchronous "conventional" high grade HLRCCRCC was present. Sections showed distinctive, low grade oncocytic neoplasms, predominantly unencapsulated, nodular, solid (one partially cystic) tumors with nested to tubular architecture. These were composed of polygonal cells with eosinophilic cytoplasm with flocculence and vacuolation, and nuclei with finely clumped to neuroendocrine-like chromatin and scattered micronucleoli. For each, the morphologic differential diagnosis included a low grade oncocytic carcinoma such as chromophobe RCC or SDHB-deficient RCC, though each case was confirmed as HLRCC (one known germline FH mutation, one with prior and synchronous multiple cutaneous leiomyomata, and one with family history of RCC and FH mutation). Given the differential, SDHB IHC performed on all three was uniformly retained. $\mathrm{FH}$ expression was lost in $2 / 3$ cases, while $2 \mathrm{SC}$ expression was induced in both examples tested (including the $\mathrm{FH}$ retained example). The cases with low grade tumors have shown no progression at 7 and 2 years, respectively.

Conclusions: We identify a novel morphologic variant of HLRCC, posing a differential diagnosis with SDHB-deficient RCC, raising intriguing questions of differential function of $\mathrm{FH}$ versus SDHB loss in tumorigenesis, and cautioning careful prospective case workup of familial tumors.

1049 Reduced Survival in Estrogen Receptor Beta Expressing Bladder Cancer: Association with Altered PTEN/mTOR Status

James Solomon, Irene Thung, David Tacha, Ahmed Shabaik, Donna Hansel. University of California at San Diego, San Diego, CA; Biocare Medical, Concord, CA.

Background: Estrogen receptor beta $(\mathrm{ER} \beta)$ is the major ER isoform expressed in bladder cancer and is proposed to associate with advanced disease and worse outcomes in women. However, the regulation of ERß in this context, and its relationship to outcomes in male bladder cancer patients, has not been well studied. We evaluated ERß expression in patients with advanced bladder cancer to determine relationship to disease progression and survival and to assess its expression with upstream PTEN/mTORC1 pathway activity, which can regulate ligand-independent ERß activity.

Design: Forty-three patients (M:F 31:12) with urothelial carcinoma (UCC) at cystectomy and 10 patients with normal urothelium were included for study. ERß $(1: 1000$, Biocare Medical), PTEN (Predilute, Dako) and P-S6 (1:1000, Cell Signaling) were analyzed by immunohistochemistry and scored as 0 (no expression) to $3+$ (intense expression). PTEN sequencing was performed using Illumina MiSeq. Pair-wise comparisons were performed using Student's t-test and Pearson correlation coefficient.

Results: Nuclear ERß expression in UCC averaged 1.6 in females and 1.4 in males $(p=0.23)$ and was present in $40 / 43$ patients throughout the tumor, whereas normal urothelium showed focal expression in basal layers of the urothelium. Increased ER $\beta$ expression was significantly associated with reduced disease-specific survival $(p=0.03)$ and was weakly correlated with increased pathological stage $(r=0.10)$. Activity of mTORC1, the mTOR complex that influences ERß phosphorylation, was evaluated by P-S6 status and was positively correlated with ERß expression $(r=0.5)$. In contrast, a negative correlation was identified for PTEN $(\mathrm{r}=-0.6)$, which is an upstream inhibitor of mTOR activity. PTEN genomic analysis revealed only 4 cases with single nucleotide base substitutions (one intronic; three exonic) and no deletions, suggesting that nonmutational events may drive reduced PTEN protein expression in this cohort

Conclusions: Altered PTEN/mTOR signaling has recently emerged as a regulator of ligand-independent ER $\beta$ activity, although the association in bladder cancer has not been studied. We have identified reduced PTEN protein and increased mTORC1 activity associated with elevated ERß expression. These findings may highlight one potential mechanism by which mTOR can negatively influence outcomes in patients with bladder cancer.

1050 Tumors Involving the Ureteral Orifice: A Clinicopathologic Analysis of 93 Cases

Keith Stevens, Adeboye O Osunkoya. Emory University, Atlanta, GA.

Background: Although tumors involving the bladder and ureter have been well described, there is very limited pathologic data specifically analyzing tumors involving the ureteral orifice (UO)

Design: A search through our Urologic Pathology files and consult cases of the senior author was performed for biopsy and resection specimens [radical cystectomy $(\mathrm{RC}) /$ cystoprostatectomy(RCP)/nephroureterectomy and bladder cuff (NUBC)] of urothelial carcinoma (UCa) involving the ureteral orifice. Patient demographics, tumor grade, laterality, variant histology and depth of invasion were documented. Follow-up data was also obtained.

Results: Ninety three cases of UCa involving the UO were identified. Sixty two (67\%) patients were male. Mean age was 71 years (range: $43-91$ years). Forty two of $93(45 \%)$ cases were invasive UCa (41 high-grade UCa; 1 low-grade UCa); $17 / 42$ (40\%) were invasive into muscularis propria. Fifty one $(55 \%)$ of the tumors were non-invasive: 22 high-grade papillary UCa, 17 low-grade UCa, and12 CIS. Tumor laterality was as follows: right side, 43 (46\% of cases); left side, 42 (45\%); bilateral, 4 (4.5\%); and in 4 cases $(4.5 \%)$ the laterality was not specified by the Urologist. Thirty eight patients underwent a radical resection: $31 \mathrm{RC} / \mathrm{RCP}$ and $7 \mathrm{NUBC}$. The resection specimens were staged as follows: for RC/RCP cases, 12 (39\%) had a stage of $\leq \mathrm{pT} 1$ ( 3 pTX, 1 pTa, 4 pT1, and 4 pTis); 7 (22\%) were pT2 (3 pT2a; 4 pT2b); 8 (26\%) were pT3 (5 pT3a; 3
pT3b), and 4 (13\%) were pT4; for NUBC: $2 / 7$ (29\%) were pTa, $2 / 7$ (29\%) were pT1, and $3 / 7(42 \%)$ were pT3. Tumor size ranged from microscopic to $8.0 \mathrm{~cm}$. Seven cases of UCa with variant differentiations were also identified ( 2 micropapillary; 2 sarcomatoid; 2 squamous and 1 glandular). Five patients had lymph node (LN) metastasis at the time of resection, and another 3 presented with LN or distant metastasis after resection (range: 4-38 months). Four patients had local recurrence after radical resection (range: 7-37 months). Although this study focused primarily on the index tumor involving the ureteral orifice, in $69 / 93(74 \%)$ cases, at least one other tumor was located at another site within the bladder.

Conclusions: This is one of the largest studies to date on tumors involving the ureteral orifice. Although this is not a site typically biopsied, $45 \%$ of cases had invasive UCa (including aggressive variants). In addition, the fact that the majority of cases (74\%) had tumors located at other sites of the bladder, emphasizes that careful examination of the ureteral orifice needs to be performed by both Urologists and Pathologist when examining cases of $\mathrm{UCa}$ of the bladder.

1051 PLZF: A Sensitive and Specific Biomarker for Yolk Sac Tumor Shana Straub, David E Burstein, Hani Katerji, Faqian Li, Qi Yang, Loralee McMahon, Guang-Qian Xiao. University of Rochester, Rochester, NY; Mount Sinai School of Medicine, New York, NY; University of Minnesota, Minneapolis, MN

Background: Germ cell tumors (GCTs) differ in their clinical behavior and management. Yolk Sac Tumor (YST) has been known to be the most commonly underdiagnosed GCT element. The currently available GCT biomarkers are only marginally sensitive and/ or specific for YST. Although the function of Promyelocytic Leukemia Zinc Finger (PLZF) protein, a transcription repressor, is well studied in spermatogenesis, expression of PLZF in GCT has not yet been reported. The aim of this study was to investigate the immunohistochemical expression and diagnostic utility of PLZF in GCTs.

Design: A total of 67 adult GCTs collected at University of Rochester Medical Center were studied, which included 62 testicular primary GCTs, 2 ovarian YSTs, 1 mediastinal YST, and 2 retroperitoneal metastatic testicular YSTs. Of the 62 testicular primary GCTs, 34 were pure GCTs (20 seminomas, 8 embryonal carcinomas (ECs), 2 teratomas, 1 choriocarcinoma, 1 monodermal carcinoid, and 2 spermatocytic seminomas) and 28 were mixed GCTs (composed of 13 ECs, 15 YSTs, 15 teratomas, 7 seminomas, and 3 choriocarcinomas in a various mixture). 35 cases contained intratubular germ cell neoplasia (ITGCN). Immunoreactivity was graded as: $<1 \%$ (negative), $1-25 \%$ (focal), $25 \%-50 \%$ (moderate), and $>50 \%$ (diffuse) staining.

Results: YST was consistently reactive with PLZF. Among the 15 testicular YSTs in mixed GCTs, all (100\%) presented with moderate to diffuse PLZF staining. PLZF reactivity was present in all the growth patterns of YST. PLZF also picked up small foci of YST intermixed/embedded in other GCT subtype elements of mixed GCT. Additionally, diffuse PLZF immunereactivity was observed in $2 / 2$ recurrent metastatic YSTs, 1/1 mediastinal YST, and 2/2 ovarian YSTs. Except spermatocytic seminoma $(n=2)$ and carcinoid $(n=1)$, in which PLZF was also diffusely expressed, all the other nonYST GCTs were completely nonreactive with PLZF, including seminoma ( $n=27)$, EC $(n=21)$, teratoma $(n=17)$, choriocarcinoma $(n=4)$, and ITGCN $(n=35)$. The sensitivity and specificity of PLZF in detecting YST was $100 \%(20 / 20)$ and $96 \%(66 / 69)$, respectively. Conclusions: Our study demonstrated that PLZF moderately to diffusely immunoreacted with all YSTs (gonadal, extragonadal and metastatic) and, except in spermatocytic semnioma and carcinoid, no immunoreactivity was observed in the other types of GCTs. In conclusion, PLZF is a highly sensitive and specific marker for YST, superior to other currently available YST biomarkers - alpha-fetoprotein and Glypican 3.

\section{Analysis of PD-L1 Expression Pattern in Advanced Bladder Cancer and Lymph Node Metastasis}

Yue Sun, Max Vaickus, Sandra Cerda, Huihui Ye. Boston University Medical Center, Boston, MA; Beth Israel Deaconess Medical Center, Boston, MA.

Background: Upregulation of the programmed death-1/programmed death ligand-1 (PD-1/PD-L1) pathway is an important mechanism developed by tumor cells to shut down host immune surveillance. Recent clinical trials demonstrated the therapeutic potential of agents that target PD-1 or PD-L1 in patients with advanced cancers. However, developing more accurate predictive biomarkers of response in late stage tumors with distance metastasis remains a challenge. It is unknown whether the primary tumors and their lymph node metastasis present the same biology in an immune therapy setting.

Design: We reviewed 21 cases of stage III/IV bladder urothelial carcinoma with 15 cases identified as pN1 and 6 cases as pN0. Primary tumor and lymph node (LN) specimens were subjected to immunohistochemistry (IHC) to evaluate PD-L1 expression (clone 5H1)

Results: PD-L1 was expressed in $33 \%$ of primary stage III/IV urothelial carcinoma (n $=21$ ). Expression patterns include: diffuse, scattered, or at tumor infiltrating border. Interestingly, 4 cases of urothelial carcinoma with focal squamous differentiation showed strong diffuse PD-L1 expression in the squamous component, while no PDL1 expression was identified in the classic urothelial carcinoma component within the same tumor. 


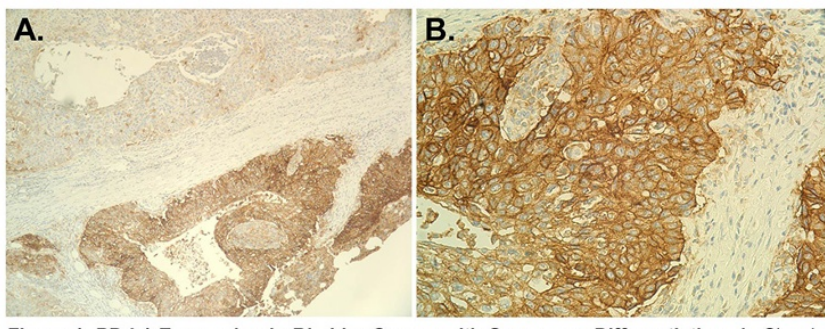

Figure 1. PD-L1 Expression in Bladder Cancer with Squamous Differentiation. A. Classic urothelial carcinoma (upper left) with a component showing squamous differentiation (lower right); B. High power shows strong PD-L1 expression in the squamous component.

We further compared PD-L1 expression in the primary tumor and metastatic LN (n $=15$ ). Three distinct PD-L1 expression groups were identified: expression in both primary tumor and metastatic LN $(20 \%)$, focal expression in the primary but not in the metastatic LN (13\%), and absence of expression in either the primary or the metastatic LN (67\%). This suggests that PD-L1 expression in the primary urothelial carcinoma is a good indicator of the expression level in the metastatic LN with $87 \%$ concordant expression rate.

Conclusions: Our result suggests that PD-L1 expression levels in the primary bladder urothelial carcinoma can be used to estimate the effect of an anti-PD-L1 treatment in patients with late stage distant metastasis. Second, 1/3 of the late stage urothelial carcinoma expresses PD-L1 with a strong diffuse staining pattern identified predominantly in the squamous differentiated component. Further studies evaluating large cohorts of patients are being investigated.

1053 Tumor Somatic Mutation Rate Predicts Response to Anti-PD-1 Therapy in RCC: A Pilot Study

Diana Taheri, Maria Angelica Mendoza Rodriguez, Mark W Ball, Stephania M Bezerra, Bert Vogelstein, Kenneth Kinzler, Janis Taube, Charles Drake, Mohamad E Allaf, George J Netto. Johns Hopkins Medical Institutions, Baltimore, MD; Isfahan University of Medical Sciences, Isfahan Kidney Diseases Research Center, Isfahan, Islamic Republic of Iran.

Background: Antibodies that block the interaction of PD-1 with PD-L1 have shown antitumor activity in solid tumors. Recently, we demonstrated objective responses in $9 / 33(27 \%)$ of renal cell carcinoma (RCC) patients treated with anti PD-1 (BMS936558) antibody. Although tumor PD-L1 expression has been associated with objective response, additional molecular and genomic predictors of response are crucially needed. The current study evaluate the role of tumor somatic mutation rate in predicting response to anti-PD-1.

Design: The current study included a total of 7 patients. 4 with complete response (CR) and 3 non-responders (NR). Archival surgical pathology material prior to anti-PD-1 treatment from all patients were retrospectively retrieved. The specimens included 7 initial radical nephrectomies and metastatic lesions in two of the patients. Representative paired tumor and benign areas were microdissected by a urologic pathologist that was blinded to treatment outcome. Three "16 gauge" cores per sample were submitted for DNA extraction and analysis by CancerXome ${ }^{\mathrm{TM}}$ method (PGDx, Baltimore, MD). Captured exome coding regions of $>20,000$ genes were sequenced at high coverage (150X) using Illumina HiSeq next generation sequencing technology. Total number of tumor somatic mutations were assessed for each of the $7 \mathrm{RCC}$ tumors without knowledge of anti-PD-1 response status.

Results: The cases included 6 clear cell RCC and 1 Xp11 translocation RCC. With the exception of one $p T 2 b$, all tumors were $\geq p T 3 a$. 6 tumors were Fuhrman grade $\geq 3$. As shown in table 1 , on average, a higher number of somatic mutation was encountered in association with CR compared to NR (mean 59.5 vs 29). Using the median number of somatic mutation as a cutoff, high somatic mutation rate ( $>37$ mutations/tumor) was significantly associated with $\mathrm{CR}(75 \%$ vs $0 \% \mathrm{CR}$; $\mathrm{p}=0.04)$. The top 4 recurrent mutations included ( $v H L, P B R M 1, S E T D 2$ and MFI2)

\begin{tabular}{|l|l|l|l|l|}
\hline \multirow{2}{*}{ Outcome } & \multicolumn{2}{|c|}{ Number of Somatic Mutation } & \multicolumn{2}{c|}{ Somatic Mutation Status* } \\
\cline { 2 - 5 } & Mean & Median & High & Low \\
\hline CR & 59.5 & 68.5 & 3 & 1 \\
\hline NR & 29 & 35 & 0 & 3 \\
\hline
\end{tabular}

\section{*p:0.04}

Conclusions: Our study is the first to assess the relationship of somatic mutations rate and response to anti-PD1 therapy in RCC. A higher rate of somatic mutations is associated with response to anti PD-1 in RCC. Our promising finding merits confirmation in larger prospective cohort.

1054 ALK Immunohitochemical Expression in Inflammatory Myofibroblastic Tumor (IMT) Using Novel D5F3 Clone in Comparison to ALK1 Clone Antibody

Diana Taheri, David J Zahavi, Maria DC Rodriguez, Abdelrazak Meliti, Neda Rezaee, Shahaboddin Dolatkhah, Yi Ning, Justin A Bishop, George J Netto, Rajni Sharma. Johns Hopkins Medical Institution, Baltimore, MD; Isfahan University of Medical Sciences, Isfahan, Islamic Republic of Iran.

Background: IMT is a rare but challenging neoplasm when occurring in urologic tract. Up to $60 \%$ of IMTs show $A L K$ (anaplastic lymphoma kinase) gene fusions resulting in ALK overexpression on IHC. A novel anti-ALK (D5F3) clone has been shown to be of superior sensitivity in the setting of $A L K$-rearranged lung adenocarcinoma. We compared the performance of D5F3 clone in detecting ALK protein expression in IMTs compared to currently utilized ALK1 clone.

Design: 25 IMTs were obtained from our surgical pathology files (2005-2015). Novel rabbit monoclonal anti-human (Clone: D5F3) and previously available mouse monoclonal anti-human (Clone: ALK1) were applied using an automated slide stainer. The percentage of immunoreactive tumor cells $(0,<5 \%, 5-50 \%,>50 \%)$ and cytoplasmic staining intensity (graded 0-3) were assessed and compared between the two antibodies. Fluorescent in-situ hybridization (FISH) studies for $A L K$ gene rearrangement were available in 5 cases.

Results: The IMTs were from various urologic (Bladder 4 and kidney 2), lung (7), gastrointestinal (4) and other sites (8). They presented 17 primary, 2 recurrent and 6 metastatic lesions. Overall, considering $>5 \%$ extent as a cut-off for positivity, D5F3 clone stained a higher number of cases compared to ALK1 [21/25 (84\%) and 18/25 $(72 \%)$, respectively, $p=0.3]$. As shown in figures 1 , significantly higher proportion of IMTs demonstrated extensive staining ( $>50 \%$ cells) with D5F3 compared to ALK1 clone $(19 / 25 \mathrm{vs} 7 / 25 ; \mathrm{p}<0.001)$. The same was true for the proportions of tumors showing high $(3+)$ intensity staining $(19 / 25$ vs $0 / 25 ; p<0.001)$. There was no difference in intensity nor extent of D5F3 staining between primary vs recurrent or metastatic IMTs.

In 5 IMTs with available FISH analysis, D5F3 IHC staining was concordant with FISH results in 4 cases ( 3 positive and 1 negative for rearrangement). Interestingly, a single IMT revealed positive IHC staining for both D5F3 and ALK1 clones but failed to show rearrangement by FISH.

Distribution of extent of Immunoreactivity by D5F3 and ALK1

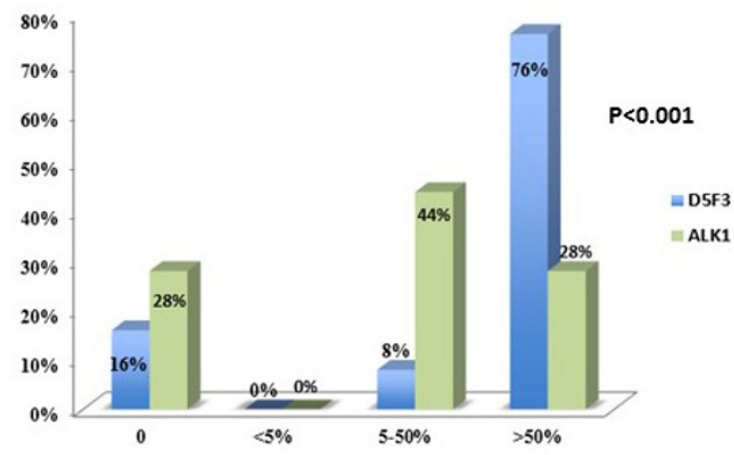

Distribution of Staining intensity by D5F3 and ALK1

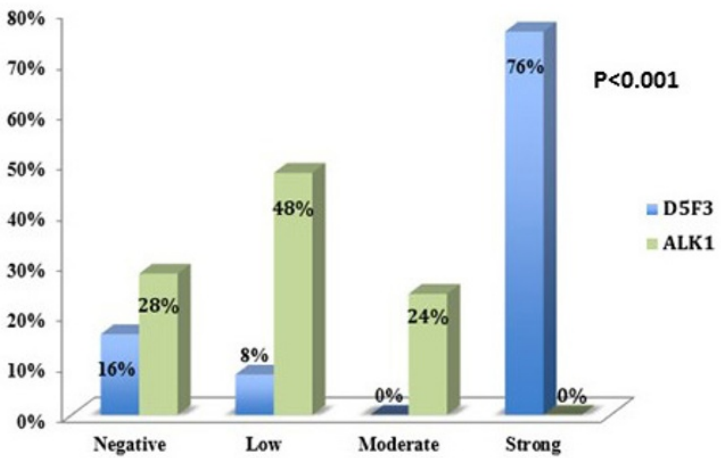

Conclusions: Novel anti-ALK rabbit monoclonal antibody (D5F3 clone) demonstarted a superior overall performance compared to currently used mouse monoclonal ALK1 clone.

1055 Type 10 Soluble Adenyl Cyclase is Significantly Overexpressed in Small Cell Prostatic Carcinoma Compared to Neuroendocrine Prostatic Carcinoma and Conventional Adenocarcinoma

Peyman Tavassoli, Francesca Khani, Jonathan H Zippin, Brian D Robinson. Weill Cornell Medical College, New York, NY.

Background: Type 10 soluble adenyl cyclase (sAC) is a ubiquitous, newly characterized enzyme that has a variety of functions based on its cellular localization which is distinct from transmembrane adenyl cyclase. We recently showed that sAC is overexpressed in prostate cancer and that it may have an important role in a signaling pathway that controls the proliferation of prostatic carcinoma cells. In the current study, we sought to compare the nuclear and cytoplasmic expression levels of sAC among benign prostatic glands, conventional prostatic adenocarcinoma (CA), neuroendocrine prostatic carcinoma (NEPC), and small cell prostatic carcinoma (SCPC) in order to evaluate its potential role in the pathogenesis of prostate cancer as well as its potential use as a prognostic marker and therapeutic target.

Design: We retrospectively identified 34 CA cases, 45 NEPC cases, and 49 SCPC cases as well as 33 benign prostate samples, all from radical prostatectomy specimens from our surgical pathology database. A tissue microarray was constructed and stained with a custom antibody against sAC (clone R52). The TMA was scanned by Aperio 
(Leica Biosystems) and visually evaluated before being analyzed by the HALO image analysis software (Indica Labs). Both cytoplasmic and nuclear staining was evaluated independently.

Results: The majority of benign and malignant prostatic glands showed at least weak cytoplasmic staining and occasional nuclear staining. There was no difference in the cytoplasmic expression level between the benign glands and CA. However, the expression level in NEPC was 2.5 times higher than in CA and benign glands ( $\mathrm{p}<$ 0.01). Similarly, SCPC had sAC expression level greater than 3 and 10 times higher than in NEPC and CA, respectively $(\mathrm{p}<0.01$ and $<0.0001$ ). When the nuclear expression level of sAC was compared among the four different groups, only SCPC showed a significantly different expression level $(\mathrm{p}<0.001)$. The average nuclear expression level in SCPC was more than 3 and 4 times higher than in NEPC and CA, respectively. Conclusions: We found that $\mathrm{SAC}$ has a different protein expression profile comparatively among CA, NEPC, and SCPC. Also, nuclear expression of sAC is significantly increased in SCPC compared to benign prostate, CA, and NEPC. These findings suggest a possible role for $\mathrm{SAC}$ in SCPC transdifferentiation and as a potential biomarker for aggressive disease. Further studies are needed to validate these findings and evaluate the role of $\mathrm{sAC}$ as a potential therapeutic target.

1056 Whole Exome Sequencing Reveals 19q13 Amplification and Actionable Mutations in Carcinosarcoma of the Bladder

Peyman Tavassoli, Bishoy S Morris Faltas, Joanna Cyrta, Juan Miguel Mosquera, Brian D Robinson, Mark Rubin. Weill Cornell Medical College, New York, NY.

Background: Carcinosarcoma of bladder is rare, mostly diagnosed at higher stages with poor prognosis. The molecular pathophysiology of carcinosarcoma of the bladder is poorly understood. We describe a unique case of bladder carcinosarcoma with phyllodes morphology and report results of whole exome sequencing (WES) for this case.

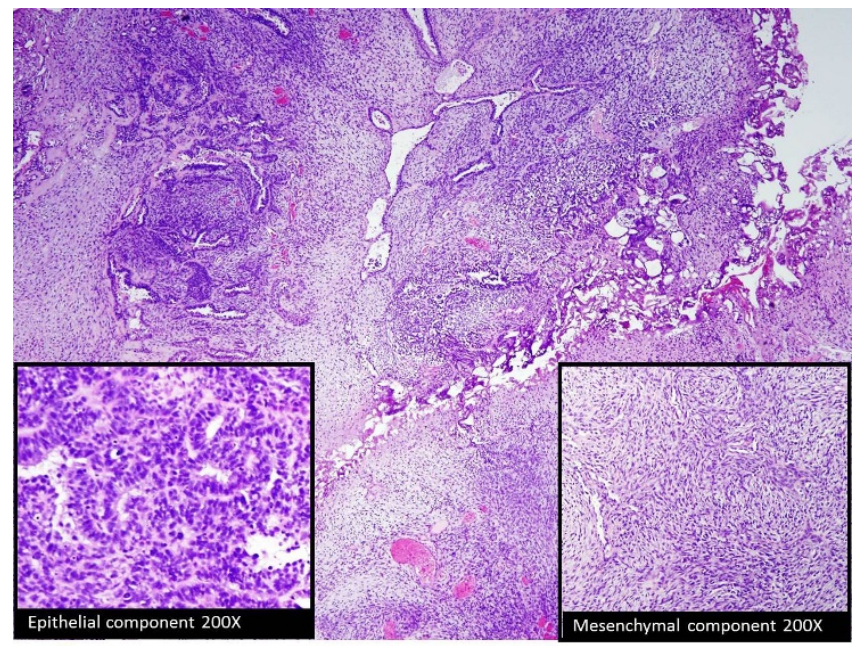

Design: A 75 y.o. male presented with urosepsis at our hospital. CT showed bilateral hydronephrosis and a bladder mass.

Results: TUR biopsy revealed malignant mesenchymal components with rhabdomyosarcoma differentiation, malignant epithelial components with glandular differentiation and no evidence of carcinoma in situ. Representative area of containing both components was selected for WES. We identified 21 mutations, including FRMDA4, FAT4, KHDC1 and FGFR2 and 223 gene amplifications. Interestingly, all the 223 amplified genes including AKT2, AURKC, MIR371, MIR372 and MIR373 are located in Ch 19q13.1-13.4 loci.

\begin{tabular}{|l|l|l|}
\hline Gene & Protein Change & Allele Frequency \\
\hline APC & G2006C & $5 \%$ \\
\hline NOTCH4 & $c .44 \_47 T G C T-$ & $76 \%$ \\
\hline PTPRD & $T 412 N$ & $5 \%$ \\
\hline NF2 & $A 388 S$ & $6 \%$ \\
\hline STK11 & $A 316 D$ & $5 \%$ \\
\hline KEAP1 & $T 330 N$ & $6 \%$ \\
\hline FGFR2 & $P 180 Q$ & $5 \%$ \\
\hline FAT4 & $P 214 A$ & $59 \%$ \\
\hline & Cytoband & Copy Number Alterations \\
\hline AKT2 & $19 q 13.1-q 13.2$ & Amplification \\
\hline AURKC & $19 q 13.43$ & Amplification \\
\hline
\end{tabular}

Conclusions: To our knowledge, this is the first report of whole-exome sequencing of bladder carcinosarcoma showing 19q13 amplification. This is seen in less than $2 \%$ of our urothelial carcinoma cohort and 5\% of TCGA bladder cohort. Amplification of this region has been reported in uterine carcinosarcoma and ependymoblastoma, both of them are biphasic neoplasms, suggesting a possible role in carcinosarcoma development. In addition, AURKC, one of the amplified genes in this region, plays a role in sarcomatoid transformation. Despite the poor prognosis of carcinosarcoma, molecular data can be helpful for exploring treatment options. We identified potential molecular targets including AKT2 and AURKC amplification, as well as FGFR2 mutation. Further studies are essential to understand the molecular pathology of bladder carcinosarcoma as a distinct entity.

1057 Higher Expression of CDKN2A Gene Is Potential Prognostic Marker for Clear Cell Renal Cell Carcinoma

Eriny Tawedrous, Samantha J Wala, Rola Saleeb, Fabio Rotondo, Qiang Ding, George $M$ Yousef. St. Michael's Hospital, Li Ka Shing Knowledge Institute, Toronto, ON, Canada; University of Toronto, Toronto, ON, Canada.

Background: Renal cell carcinoma is the most common lethal genitourinary cancer; clear cell renal cell carcinoma (ccRCC) is the most common subtype (75\%) of renal cell carcinoma and can exhibit varying degrees of aggressiveness, therefore predicting the prognosis is of crucial importance towards personalized patient's management plan Design: We investigated the prognostic value of the cyclin-dependent kinase inhibitor 2A $(C D K N 2 A)$ gene expression, which is encoded on chromosome 9p21, in 294 primary ccRCC cases by immunohistochemistry on tissue microarray. We compared its expression with clinicopathologic parameters and patients' survival. We also validated our results at the mRNA level on an independent set (415) from The Cancer Genome Atlas (TCGA).

Results: There was a significant association between $C D K N 2 A$ expression and tumor size, with higher expression associated with smaller tumor size $(\mathrm{p}<0.001)$. As a dichotomous variable (positive vs. negative expression), there was a significant association between $C D K N 2 A$ expression and tumor grade, with positive expression associated with lower tumor grades $(\mathrm{p}<0.001)$. Molecular pathway assessment has shown that $C D K N 2 A$ is implicated in cell cycle, apoptosis and p53 pathway. In the univariate analysis, positive expression was associated with significantly longer diseasefree survival $(\mathrm{HR}=0.46 ; \mathrm{p}=0.006)$. This was maintained in the multivariate analysis $(\mathrm{HR}=0.44 ; \mathrm{p}=0.005)$. Kaplan-Meier survival analyses showed that positive $C D K N 2 A$ expression was associated with statistically significant increase in disease-free survival $(\mathrm{p}=0.007)$. We independently validated our results on 415 patients from the TCGA cohort which similarly showed a significant increase of the overall survival with higher $C D K N 2 A$ expression levels ( $\mathrm{P}=0.05)$; with median months of survival ( 76.97 vs. 42.44$)$. Conclusions: Our results indicated that $C D K N 2 A$ is a potential prognostic marker for ccRCC that may give clinicians an extra tool to guide individual patient's management.

1058 Isolated 9p21 Deletion Detected by UroVysionTM Fluorescence InSitu Hybridization in Urine Samples: A Study of 69 Cases with Emphasis on Clinicopathological Features and Outcome

Wei Tian, Eric Kroman, Wendy L Flejter, Rajal Shah. Miraca Life Sciences, Irving, TX. Background: Fluorescence in situ hybridization (FISH) analysis of urine samples for aneuploidy of 3, 7, and 17 and deletion of 9p21 has high sensitivity and specificity for diagnosing urothelial carcinoma (UC) for both initial diagnosis and subsequent monitoring of tumor recurrence. A chromosome 9 deletion is considered an early genetic alteration in bladder cancer, but little is known about the clinicopathological spectrum and significance of isolated $9 \mathrm{p} 21$ deletion.

Design: All urine samples processed from 2/2013 to 6/2014 were searched for UroVysion $^{\mathrm{TM}}$ testing from our departmental database. Isolated $9 \mathrm{p} 21$ deletion abnormality was defined as 12 or more cells without $9 \mathrm{p} 21$ gold signals in the absence of aneuploidy of chromosome 3,7 , and 17. Clinical presentations, urine cytology diagnoses and follow up information were collected.

Results: Of 6146 urine samples, 1613 had UroVysion ${ }^{\mathrm{TM}}$ FISH results available; 69 $(1.1 \%)$ had isolated 9 p21 deletion. The mean age was 68 years (range: $19-96$ years), with a M: F ratio of $2.6: 1$. Twenty five $(36 \%)$ presented with a history of urothelial carcinoma, and $44(64 \%)$ for urine work up related to: hematuria 38 , urinary tract infection 3 , urinary retention 1 , nocturia 1 , and stone 1 . Fifty five $(80 \%)$ were voided and $14(20 \%)$ were instrumented urine. Urine cytology findings included: benign 14 $(20 \%)$; mild atypia favor reactive $35(50 \%)$; atypical $19(28 \%)$, and FISH only 1 . The average percentage of abnormal cells with $9 \mathrm{p} 21$ deletion was $28.6 \%$ (range: $6.1-100 \%$ ). Follow-up information was available for $31(45 \%)$ patients with a mean length of 10.5 months (range: 1-26 months). Abnormalities were observed in $6(19 \%)$ : 2 continued to demonstrate 9p21 deletion by FISH, 2 atypical urine cytology and 2 developed low grade tumor recurrence confirmed by biopsy.

Conclusions: Isolated 9p21 deletion is a relatively uncommon UroVysion ${ }^{\mathrm{TM}}$ abnormality. Cytology samples are often unremarkable and lack characteristic abnormalities. Patients often tend to lack evidence of disease on follow up examination. Our preliminary results suggest that isolated $9 \mathrm{p} 21$ deletion, without other chromosomal aneuploidy, may represent an early molecular abnormality or field effect of urothelial mucosa without clinically manifested disease. The result should be interpreted cautiously and should be confirmed with a repeat examination, specifically in the absence of clinical disease.

1059 Identifying Additional Lymph Nodes In Radical Prostatectomy Pelvic Lymph Node Specimens

Jessica Tracht, Win S Lai, Soroush Rais-Bahrami, Jennifer B Gordetsky. University of Alabama at Birmingham, Birmingham, AL.

Background: Pelvic lymphadenectomy has prognostic and therapeutic implications in prostate cancer. Pelvic lymphadenectomy specimens are fatty and identification of lymph nodes can be difficult during the grossing process. We investigated the utility of submitting the entire lymph node packet for examination.

Design: We retrospectively examined 59 radical prostatectomy cases with lymph node dissections at our institution between 2012 and 2015. A new method requiring the total submission of lymph node packets was introduced in 2014 . We assessed the difference in lymph nodes identified, number of blocks submitted, and the proficiency of finding lymph nodes based on PGY level. 
Results: 29 cases using old grossing methods and 30 cases were identified using the new lymph node grossing method. The mean age and PSA were $64.9 \mathrm{vs} 59.7$ years and $14.4 \mathrm{vs} 8.8 \mathrm{ng} / \mathrm{mL}$ for the old and new methods ( $\mathrm{p}=0.0051$ and $\mathrm{p}=0.13$ ), respectively. Pathologic T stage, N stage, and grade were not significantly different between the two groups. Significantly more lymph nodes were found when total lymph node packets were submitted; mean 14.1 vs 8.7 ( $\mathrm{p}=0.0074)$. Significantly more blocks were required using the new grossing method; mean 13.53 vs $6.9(p<0.001)$. Submitting the entire lymph node packet resulted in a mean of 3 additional lymph nodes being detected per specimen. No significant difference in the number of lymph nodes found was noted by comparing all PGY levels of residents processing the case $(p=0.33)$. However, using the new grossing technique, the percent increase in lymph node count was significantly lower when comparing upper level residents vs junior level residents $(1,2$ vs 3,4 : $p<0.001$ and $1,2,3$ vs $4: \mathrm{p}=0.014)$. PGY4 residents also found significantly fewer additional lymph nodes using the new grossing technique compared to the others PGY levels $(\mathrm{p}=0.04)$. Conclusions: Entire lymph node packet submission significantly increases the total number of lymph nodes identified in pelvic lymphadenectomy specimens submitted during radical prostatectomy. The experience of the grosser, as measured by PGY level, also increases the augmented percentage of lymph node yield. As more blocks are required for this new grossing method, there will likely be an increased expense in specimen processing.

\section{Increased Proliferative Rate and PTEN Loss in Prostate Cancer Are} Correlated and Both Associated with Risk of Recurrence in Multivariate Models

Maria S Tretiakova, Wei Wei, Carlos L Morais, Ziding Feng, Jesse K McKenney, Jeff Simko, Dean Troyer, Lawrence True, Funda Vakar-Lopez, Ladan Fazli, Peter S Nelson, Daniel Lin, James D Brooks, Tamara Lotan, Canary Prostate Investigators. University of Washington, Seattle, WA; Canary Foundation, Palo Alto, CA; John Hopkins University, Baltimore, MD.

Background: PTEN is the most commonly lost tumor suppressor gene in primary prostate cancer (PC), and its loss is associated with poor clinical outcomes. Increased cellular proliferation has also been associated with poor outcomes in PC. Here, we examined the relationship between PTEN loss and Ki-67 expression in primary PC treated by prostatectomy.

Design: 1275 radical prostatectomies from the Canary Retrospective PC TMA cohort were analyzed for PTEN loss by immunohistochemistry (IHC) using a genetically validated automated protocol and scoring system to assess homogeneous (in all sampled tumor glands) or heterogeneous (in a subset of sampled tumor glands) PTEN loss. IHC for Ki-67 was performed on the same TMAs and scored by automated image analysis (Aperio). Association of PTEN loss and Ki-67 was assessed by Kruskal-Wallis test, and multivariate Cox proportional hazard model was used to correlate factors with recurrence free survival (RFS).

Results: Ki-67 expression was associated with PTEN loss, with a median Ki-67 IHC rate of $2.98 \%$ of tumor cells in cases with intact PTEN, compared to $3.38 \%$ and $4.76 \%$ in cases with heterogeneous and homogeneous PTEN loss, respectively $(\mathrm{p}=0.0001)$. In multivariate Cox model for RFS, homogeneous PTEN loss (HR=2.09 against PTEN intact, 95\% CI: 1.51-2.9), Ki-67>5\% (HR=1.33, 95\% CI: 1.04-1.7), surgical margin positivity, Gleason score, seminal vesicle invasion and pre-operative PSA all remained significant.

\begin{tabular}{|l|l|l|l|l|l|}
\hline Factor & Comparison & $\begin{array}{l}\text { Hazard } \\
\text { Ratio }\end{array}$ & $\begin{array}{c}95 \% \\
\text { LCL }\end{array}$ & $\begin{array}{c}95 \% \\
\text { UCL }\end{array}$ & P-value \\
\hline PTEN (IHC) & Homo-loss vs Intact & 2.09 & 1.51 & 2.90 & $<.0001$ \\
\hline & $\begin{array}{l}\text { Hetero-loss vs } \\
\text { Intact }\end{array}$ & 1.20 & 0.87 & 1.67 & 0.27 \\
\hline Ki-67 & $>=5 \%$ vs $<5 \%$ & 1.33 & 1.04 & 1.70 & 0.03 \\
\hline Margin & $\begin{array}{l}\text { Positive vs } \\
\text { Negative }\end{array}$ & 1.75 & 1.37 & 2.23 & $<.0001$ \\
\hline Seminal vesicle invasion & $\begin{array}{l}\text { Positive vs } \\
\text { Negative }\end{array}$ & 2.17 & 1.47 & 3.21 & $<.0001$ \\
\hline Gleason score & $3+4$ vs 6 & 1.26 & 0.93 & 1.70 & 0.14 \\
\hline & $4+3$ vs 6 & 1.82 & 1.27 & 2.59 & 0.001 \\
\hline & $>=8$ vs 6 & 1.62 & 1.10 & 2.39 & 0.01 \\
\hline Log (pre-op PSA) & 1 unit increase & 1.59 & 1.33 & 1.91 & $<.0001$ \\
\hline
\end{tabular}

Conclusions: We found that $\mathrm{Ki}-67$ as a continuous variable is strongly associated with both homogeneous and heterogeneous loss of PTEN by validated and automated IHC assay. Homogeneous PTEN loss and Ki-67 > 5\% are both associated with decreased RFS in primary prostate cancers after adjusting for other clinical factors.

1061 Prostate Cancer Gleason Grade 4 Pattern Heterogeneity in Men on Active Surveillance Who Undergo Delayed Prostatectomy

Patricia Troncoso, John W Davis, Elsa Li Ning Tapia, Ina Prokhorova, John F Ward, Louis L Pisters, Mary Achim, Christopher J Logothetis, Jeri Kim. The University of Texas MD Anderson Cancer Center, Houston, TX.

Background: Active surveillance (AS) for men with clinically localized Gleason score (GS) 7 prostate cancer is controversial due to the uncertainty about its biological potential. Limited information exists about the morphologic heterogeneity of Gleason grade (GG) 4 in patients (pts) on AS. The morphologic patterns of GG4 are poorly formed glands (PFG), fused glands (FG), and cribriform. Studies have reported their prevalence in several patient populations and the association of the cribriform pattern with aggressive biology.
Design: We evaluated the GG4 patterns in the prostate biopsies (BXs) and radical prostatectomy specimens (RPS) of pts enrolled in a prospective cohort AS study (currently $>1000$ pts enrolled) who underwent prostatectomy following disease reclassification to determine the prevalence of GG4 PFG/FG and cribriform patterns in the prostate BXs and RPS.

Results: Between 2009 - 2015, forty-four pts underwent delayed prostatectomy. The median age at prostatectomy was 65 yrs (48-79) and the median time on AS was 26 months (9-77). The median number of BXs/pt was 3.5. Overall, the highest GS in the BXs was $6,7(3+4), 7(4+3)$, and $\geq 8$ in $7,25,6$, and 10 pts, respectively. PFG/ FG exclusively and PFG/FG plus cribriform pattern were observed in at least one surveillance BX in 29/37 (78\%) and 8/37 (22\%) pts with a GG4 component, respectively. The cribriform pattern was present in 2 BXs with GS $7(3+4)$ and 6 with GS $\geq 7(4+3)$. Thirty-one RPS (74\%) had single (27) or multiple (4) tumor foci with a cribriform component. RPS with tumor foci with a cribriform component had a greater median total tumor volume $(1.006 \mathrm{cc})$ than those with PFG/FG $(0.525 \mathrm{cc})$, a higher GS $(\geq 7 / 4+3$ : $48 \%$ vs $9 \%$ ) and more frequent non-organ confined disease ( $48 \%$ vs $9 \%)$. Tumor foci of peripheral zone origin with a cribriform component were smaller $(0.384 \mathrm{cc})$ than those of transition zone origin $(1.60 \mathrm{cc})$.

Conclusions: Cribriform pattern was present in $22 \%$ of BXs of patients on active surveillance at the time of disease reclassification and it was predominantly observed in BXs with a dominant Gleason grade 4 . The presence of cribriform pattern in the radical prostatectomy specimen was associated with adverse pathologic features. Further studies are warranted to evaluate the biological and clinical significance of the heterogeneity of the Gleason grade 4 patterns in patients on active surveillance.

1062 Eosinophilic Solid and Cystic Renal Cell Carcinoma (ESC RCC): A Novel Entity with Distinct Molecular Karyotype

Kiril Trpkov, Hatem Abou-Ouf, Ondrej Hes, Jose I Lopez, Gabriella Nesi, Eva Comperat, Mathilde Sibony, Daniel Berney, Isabela W Cunha, Adeboye O Osunkoya, Neriman Gokden, Ming Zhou, Tarek Bismar, Jesse K McKenney. University of Calgary, Calgary, Canada; Charles University, Pilsen, Czech Republic; Cruces University Hospital, Bizkaia, Spain; Careggi Hopsital, Florence, Italy; Pitié-Salpêtrière Hospital, Paris, France; Hopital Cochin, Paris, France; Barts Cancer Center, London, United Kingdom; AC Camargo Cancer Center, Sao Paulo, Brazil; Emory University School of Medicine, Atlanta, GA; Univeristy of Arkansas, Little Rock, AR; New York University, New York City, NY; Cleveland Clinic, Cleveland, $\mathrm{OH}$.

Background: ESC RCC has been recently described as a unique and indolent renal neoplasm, found only in females with and without Tuberous Sclerosis Complex (TSC). Although ESC RCC has distinct morphology and frequent CK20 reactivity, its molecular karyotype has been analysed in only a limited number of cases.

Design: Clinical and pathology data were retrieved from multiple institutions on 14 ESC RCC cases. Molecular karyotyping was performed in 13 cases (12 with informative result). Genomic DNA was extracted from FFPE samples following manufacturer's protocol and was subjected to OncoScan FFPE MIP based assay. Data were analyzed using Nexus Express for OncoScan Copy Number software.

Results: All 14 ESC RCC were found in female patients without clinical features of TSC. Median age was 53.5 years (range 44-74). Grossly, all tumors were yellow-gray and had a solid and cystic (12) or solid appearance (2). Median tumor size was $30 \mathrm{~mm}$ (range 12-135). Histologically, all tumors showed the typical features of ESC RCC. CK20 was positive in $11 / 14(79 \%)$ of cases. Stage pT1 (1a in $10,1 \mathrm{~b}$ in 3 ) was found in 13 of 14 patients; only 1 patient had stage pT3a. 12 of 14 patients with available follow-up were alive and without evidence of disease progression, after a follow-up from 6 to 130 months (median: $42 \mathrm{mo}$; mean $50 \mathrm{mo}$ ). The most frequent copy number $(\mathrm{CN})$ gains, $\mathrm{CN}$ losses, and $\mathrm{LOH}$ alterations are listed (in order of frequency):

CN gains: 16p13.3-16q23.1 (33-67\%), 7p21.2 -7q36.2 (42-50\%), 13q14.2 (33\%), $19 \mathrm{p} 12(33 \%)$

CN losses: Xp11.21 (42\%), 22q11.23 (33\%)

LOH: 16p11.2-11.1 (75\%), Xq11.1-13.1 (75\%), Xq13.1-21.1 (33\%), 11p11.2-11.11 (33\%) 9q21.1-22.2 (33\%), 9 q33.1 (33\%)

Of note, LOH was found at TSC1 in 33\% od samples, while TSC 2 showed $\mathrm{CN}$ gains in $42 \%$ of samples.

Conclusions: ESC RCC is a novel renal tumor with indolent behaviour, which also shows unique molecular karyotype, distinct from the other currently recognized renal tumors. Most common molecular karyotype alterations in ESC RCC include CN gains at 16p13.3-16q23.1 and 7p21.2 -7q36.2 and LOH at 16p11.2-11.1 and Xq11.1-13.1.

1063 The Depth of Tumor Invasion Is Superior to the AJCC/UICC Staging System to Predict Patient Outcomes after Radical Cystectomy: A Proposal for a New Staging System

Toyonori Tsuzuki, Toshinori Nishikimi, Yukiko Mori, Naoto Sassa, Masashi Kato, Shin Yamada, Kuniaki Tanaka, Satoru Takahashi, Yasushi Yoshino, Ryohei Hattori, Momokazu Gotoh. Japanese Red Cross Nagoya Daini Hospital, Naogya, Japan; Nagoya City University, Nagoya, Japan; Nagoya University Hospital, Naogya, Japan; Japanese Red Cross Nagoya Daiichi Hospital, Naogya, Japan; Okazaki Municipal Hospital, Okazaki, Japan; Kariya General Hospital, Kariya, Japan.

Background: AJCC/UICC staging system is the gold standard to predict patient outcomes. However, many studies report that pT staging (pT2 vs. pT3) may not be a useful prognostic factor. In addition, a recent study showed that pathological criteria of distinction between pT2 and pT3 are variable among pathologists.

Design: We retrospectively evaluated 137 bladder cancer patients who underwent radical cystectomy at the hospitals that the authors are affiliated with, and reviewed all the HE stained slides. No patients received neoadjuvant chemotherapy. The depth of tumor invasion was measured from the normal urothelium to the deepest invaded lesion. Data on the patients' age, depth of tumor invasion $(<1.0 \mathrm{~mm}$ vs. $\geq 1.0 \mathrm{~mm}$ ), pT stage (pT2 vs. 
pT3), 1973 WHO grade (G2 or G3), and lymph node status (pN0 vs. pN1) were analyzed. The patient characteristics were analyzed using the Fisher exact test. Multivariate Cox proportional hazard regression models were developed to predict the progression-free survival (PFS), cancer-specific survival (CSS), and overall survival (OS).

Results: The median age of the patients was 71 years (range, 35-87 years). The median follow-up period was 32 months (range, 1-247 months). A total of 72 patients developed recurrence, 55 died of the disease, and 14 died of other causes. Pathological characteristics were as follows: the median depth of tumor invasion was $10.80 \mathrm{~mm}$ (range 2.57-37.14 mm), pT (pT2:pT3 = 45:92), 1973 WHO grade (G2:G3 = 31:106). On univariate analysis, the depth of tumor invasion was associated with PFS $(p=.0001)$, CSS $(p=.0343)$, and OS $(p=0.0263)$. In contrast, $p$ T staging was not associated with PFS $(p=.0607)$, CSS $(p=.4891)$, or OS $(p=.6125)$. On multivariate analysis, the depth of tumor invasion was significantly associated with PFS $(p=.0045)$ and OS (p $=.0200)$. Lymph node status was significantly associated with CSS $(p=.0004)$ and OS $(p=.0020)$. There was a trend for depth of tumor invasion to be associated with CSS, but it was not statistically significant $(\mathrm{p}=.0521)$. pT staging was not associated with PFS $(p=.6781), \operatorname{CSS}(p=.5713)$, or OS $(p=.4472)$

Conclusions: The depth of tumor invasion is a more useful prognostic factor for PFS, $\mathrm{CSS}$, and OS in bladder cancer than $\mathrm{pT}$.

1064 Presence of Hormone Resistant Intraductal Carcinoma of the Prostate after Neoadjuvant Hormone Therapy Is a Significant Prognostic Factor for High Risk Prostate Cancer in Radical Prostatectomy Patients Toyonori Tsuzuki, Masashi Kato, Kyosuke Kimura, Ryo Ishida, Naoto Sassa, Akitoshi Fukatsu, Ryohei Hattori, Momokazu Gotoh. Japanese Red Cross Nagoya Daini Hospital, Nagoya, Japan; Nagoya University Hospital, Nagoya, Japan; Nagoya Medical Center, Naogya, Japan; Komaki City Hospital, Komaki, Japan; Japanese Red Cross Nagoya Daiichi Hospital, Naogya, Japan.

Background: The presence of intraductal carcinoma of the prostate (IDC-P) is an adverse prognostic factor, not only of prostate-specific antigen (PSA) failure but also of cancer-specific survival (CSS) and overall survival (OS) for patients after radical prostatectomy (RP). We aimed to evaluate whether the presence of IDC-P in needle biopsy (NB) and RP specimens is also an adverse prognostic factor for CSS and OS of patients treated with neoadjuvant hormone therapy (NHT).

Design: We retrospectively evaluated 152 high-risk prostate cancer patients treated with NHT prior to RP between 1991 and 2005 in the hospitals that the authors were affiliated with, and reviewed slides of their NB and RP specimens. Data on serum PSA level, Gleason score on NB (bGS $>8$ or not), cT stage (cT2 or higher), positive cancer core rate on $\mathrm{NB}(>66.7 \%$ or not), maximum cancer extension ratio in $\mathrm{NB}(>$ $90 \%$ or not), presence of Gleason pattern 5 (G5) on NB, and presence of IDC-P in NB and RP specimens were analyzed. Patient characteristics were analyzed using Fisher's exact test. Fine and Gray's model and Cox proportional hazard regression models were developed to predict CSS and OS, respectively.

Results: The median age of the patients was 68 years. The median follow-up period was 109 months. An IDC-P component was detected in 102 patients. Twenty-eight patients died of the disease and 12 patients died of other causes. Thirty-nine patients had IDC-P in both NB and RP specimens, 30 patients had IDC-P in only RP specimens, 15 patients had IDC-P in only NB specimens, and 39 patients did not have IDC-P. In Fine and Gray's model, the presence of IDC-P in both, NB and RP specimens was significantly associated with CSS $(\mathrm{p}=0.0035)$. In univariate analysis, presence of IDC-P $(\mathrm{p}<$ $0.0001)$, cT $(p=0.0047)$, positive core rate $(p=0.0072)$, maximum cancer extension $(p=0.0121)$, and G5 $(p=0.045)$ were significantly associated with OS. In multivariate analysis, only the presence of IDC-P $(p=0.0005)$ was significantly associated with OS. Conclusions: The presence of IDC-P in both, NB and RP specimens is an independent prognostic factor for both CSS and OS of patients treated with NHT. The presence of IDC-P in both, NB and RP specimens after NHT can be a useful marker to predict patients' clinical outcomes.

\section{Overexpression of the Long Non-Coding RNA SChLAP1} Independently Predicts Lethal Prostate Cancer

Aaron Udager, Arul M Chinnaiyan, Joshua Petimar, Xuhong Cao, Felix Y Feng, Massimo Loda, Thomas Ahearn, Philip W Kantoff, Lorelei A Mucci, Rohit Mehra. University of Michigan Health System, Ann Arbor, MI; Michigan Center for Translational Pathology, Ann Arbor, MI; Dana-Farber Cancer Institute, Boston, MA; Harvard T. H. Chan School of Public Health, Boston, MA.

Background: The long non-coding RNA (lncRNA) SChLAP1 is overexpressed in a subset of prostate cancers and associated with aggressive disease. We previously showed that SChLAP1 in situ hybridization (ISH) in formalin-fixed, paraffin-embedded (FFPE) tissues independently predicts biochemical recurrence after radical prostatectomy in clinically localized prostate cancer. In this study, we sought to evaluate the association between SChLAP1 ISH and the development of lethal prostate cancer.

Design: SChLAP1 ISH was performed on tissue microarrays of archival FFPE tissue from a large prospective cohort of American men with prostate cancer $(n=937)$, for whom long-term follow-up data are available. SChLAP1 expression was scored semiquantitatively (ISH score range $=0-400$ ), and patients were stratified by high (ISH score $\geq 100$ ) and low (ISH score $<100$ ) SChLAP1 expression. Association between SChLAP1 expression and selected clinicopathologic parameters was examined, and hazard ratios (HR) and 95\% confidence intervals (CI) for the association between SChLAP1 expression and time to development of lethal prostate cancer (defined as cancer-related death or metastases) were estimated by multivariate Cox regression analysis.

Results: 89 patients $(9.5 \%)$ demonstrated high SChLAP1 expression, which was positively associated with high-risk clinicopathologic factors, including high Gleason score, high pathologic and clinical stage, and PTEN IHC status (P-value < 0.005). High SChLAP1 expression was strongly associated with lethal prostate cancer in patients with non-advanced clinical tumor stage (i.e., less than $\mathrm{T} 3 ; \mathrm{HR}=2.1 ; 95 \% \mathrm{CI}$ $=1.1-3.7$ ) and relatively low-grade tumors (i.e., Gleason score $\leq 7 ; \mathrm{HR}=4.0 ; 95 \% \mathrm{CI}$ $=1.7-9.3$ ). Furthermore, in the subset of patients treated with radical prostatectomy, high SChLAP1 expression was strongly associated with lethal prostate cancer at the multivariate level - independent of age, Gleason score, pathologic stage, and PTEN IHC status $(\mathrm{HR}=2.2 ; 95 \% \mathrm{CI}=1.1-4.1)$.

Conclusions: Overexpression of the lncRNA SChLAP1, as assessed by ISH, independently predicts lethal prostate cancer in a large cohort of American men with long-term follow-up. Thus, SChLAP1 ISH is a promising biomarker for identifying patients at increased risk for lethal prostate cancer progression, who may benefit from adjuvant radiation or hormone therapy.

1066 Recurrent Alterations of Hippo Signaling Pathway Genes in Mucinous Tubular and Spindle Cell Carcinoma (MTSCC) of Kidney

Aaron Udager, Jesse K McKenney, Pankaj Vats, Fengyun Su, Xuhong Cao, Jincheng Pan, Rui Wang, Javed Siddiqui, Alon Z Weizer, Khaled Hafez, J Stuart Wolf, Ganesh Palapattu, Saravana M Dhanasekaran, Arul M Chinnaiyan, Rohit Mehra. University of Michigan Health System, Ann Arbor, MI; Michigan Center for Translational Pathology, Ann Arbor, MI; Cleveland Clinic, Cleveland, OH; First Affiliated Hospital, Sun Yat-Sen University, Guangzhou, China.

Background: MTSCC is a recently recognized and relatively rare subtype of renal cell carcinoma (RCC) characterized by mixed epithelioid and spindle cell components at the morphologic level. Relatively rare but unique variants of RCC, like MTSCC, have not been robustly interrogated at the molecular level. Hence, we characterized a multi-institutional cohort of MSTCC by integrative next-generation sequencing (NGS) to identify recurrent alterations.

Design: Available cases of MTSCC were identified retrospectively from the surgical pathology records database at two large academic institutions, comprising a discovery set $(n=4)$ and validation set $(n=5)$. Diagnoses were confirmed by expert genitourinary pathologists at each institution, and representative formalin-fixed, paraffin-embedded (FFPE) material from all the cases were utilized for whole exome and transcriptome NGS with an Illumina HiSeq 2000.

Results: Mutation analysis with the exome data revealed recurrent somatic alterations of tumor suppressor genes of the Hippo signaling pathway in three of four cases in the discovery set (PTPN14, SAV1, and NF2). Intriguingly, additional somatic mutations in Hippo pathway genes were also discovered in three of five cases in the validation set (PTPN14, FAT1, and HIPK2). In addition, copy number plots generated from the exome data demonstrated monosomy of chromosomes $1,4,5,8,9,13,14,15$, and 22 in all cases. Integrating the mutation and CNV data revealed homozygous loss of these critical genes, a molecular status that might define MTSCC. While no recurrent gene fusions were identified in the transcriptome data, comparative analysis with other RCC gene expression profiles enabled the discovery of a MTSCC gene signature.

Conclusions: Integrative whole exome and transcriptome NGS of MTSCC reveals recurrent copy number alterations and somatic mutations of Hippo signaling pathway genes. These data suggest that Hippo pathway dysregulation may be a fundamental causal event in the pathogenesis of MTSCC, a finding that may have diagnostic and therapeutic implications for this rare RCC subtype. Small molecule inhibitors of YAP (a key downstream component of the Hippo pathway), such as verteporfin, present an attractive opportunity for targeted therapy in the rare MTSCC patients with sarcomatoid differentiation or metastasis.

\section{Predictive Value of Intraductal Carcinoma and Cribriform} Architecture in Prostate Biopsies of Patients Treated by Radiotherapy

Theodorus H Van der Kwast, Jure Murgic, Melania Pintilie, Michelle Downes, Michael Fraser, Rob G Bristow. University Health Network, Toronto, ON, Canada; Princess Margaret Cancer Centre, Toronto, ON, Canada.

Background: Intraductal carcinoma (IDC) and cribriform architecture (CA) are increasingly associated with aggressive disease and poor clinical outcome of prostate cancer, but few studies were performed on prostate biopsies.

Design: Records of 379 patients with localized prostate cancer treated in a single center from 2005 to 2012 with contemporary image-guided dose-escalated radiotherapy were retrospectively reviewed. Original diagnostic prostate biopsy slides were reviewed for ISUP 2005 modified Gleason grading and scored for presence of IDC and/or CA. The impact of pathological features and other pre-treatment and treatment-related factors on freedom from biochemical failure (FFBF) and freedom from metastasis (FFM) was evaluated.

Results: IDC and/or CA was present in $19.3 \%$ of biopsies and was significantly associated with clinical T-stage, Gleason score, baseline PSA level, NCCN risk group, and higher percentage of positive biopsy cores (all $\mathrm{p}<0.0001$ ). After a median follow-up of 63 months, 39 and 10 patients experienced biochemical failure and distant metastasis, respectively. In univariable analysis, presence of IDC and/or CA was associated with decreased FFBF (HR $4.1(95 \% \mathrm{CI}: 2.2-7.8), \mathrm{p}<0.0001)$ and FFM $(\mathrm{HR}=4.7$ (95\%CI: 1.4-15.6), $\mathrm{p}=0.013)$. In multivariable analysis, IDC and/or CA were associated with worse FFBF $(\mathrm{HR}=2.79(1.42-5.5), \mathrm{p}=0.003)$ together with $\mathrm{NCCN}$ risk category $(\mathrm{HR}=6.75(95 \% \mathrm{CI}: 2.78-16.34), \mathrm{p}=<0.0001)$ and Gleason score (overall $\mathrm{p}$-value $<0.0001)$. Among patients with intermediate risk prostate cancer, presence of IDC and/or CA was the only significant variable in the multivariable model for FFBF $(\mathrm{HR}=3.0(95 \% \mathrm{CI}: 1.37-6.58) \mathrm{p}=0.0062)$ and was able to further stratify GS $7(4+3)$ patients $(\mathrm{HR}=3.09(95 \% \mathrm{CI}: 0.99-9.6) \mathrm{p}=0.051)$.

Conclusions: The presence of IDC and/or CA in prostate biopsies is an unfavourable factor additional to Gleason score in intermediate risk patients treated with contemporary radiotherapy. Reporting of IDC / CA in conjunction with biopsy Gleason score is recommended. 
1068 Better Treatment of Metastases Cannot Fully Explain Prostate Cancer Survival Improvements Associated with PSA Screening

Mitchell S Wachtel, Thomas Nelius, Allan Haynes, Werner T de Riese. Texas Tech University Health Sciences Center, Lubbock, TX.

Background: Some assert the prostate cancer mortality decline that occurred after introducing PSA screening was due partly to treatment improvements for metastatic cancer. We hypothesized that Surveillance, Epidemiology, and End Results (SEER) data would assess this notion and check for a change in survival after Docetaxel was introduced in 2004.

Design: A SEER 18 registries search obtained data for Black (B) and White (W) $50+\mathrm{y}$ men with metastases at histologic diagnosis of prostatic adenocarcinoma. The CMPRSK package of R 3.2.2 estimated, for 50-74y B, 75+y B, 50-74y W, and 75+y W, cumulative incidence rates and variances for prostate-cancer and other-cause death, $2 y$ for 1988-2010, 5y for 1988-2007. Joinpoint 4.1.1 compared age groups via coincidence and parallelism tests and produced estimates and $95 \%$ CI of annual percent changes (APC), joinpoints, and average APC (AAPC).

Results:
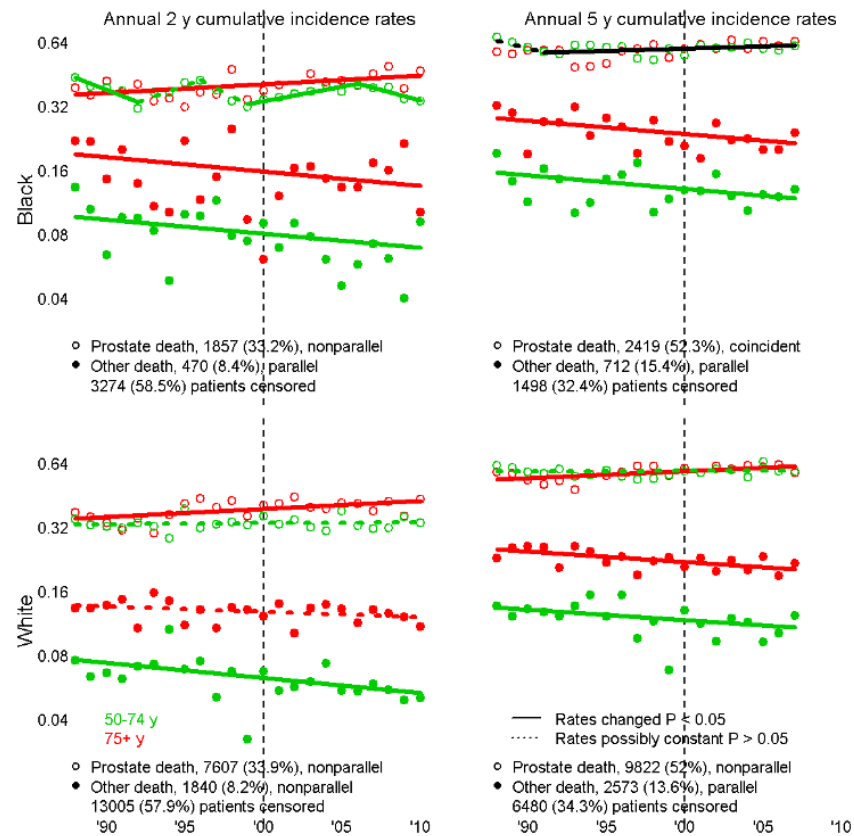

For 5y B prostate-cancer analyses, 50-74y and $75+\mathrm{y}$ had the same rates $(P>0.05$, coincidence test). For remaining prostate-cancer and $2 \mathrm{y} \mathrm{W}$ other-cause analyses, $50-74 \mathrm{y}$ and $75 \mathrm{y}$ APC differed $(\mathrm{P}<0.05$, parallelism tests). For remaining other-cause analyses, $50-74 \mathrm{y}$ and $75 \mathrm{y}$ APC were the same ( $\mathrm{P}>0.05$, parallelism tests). APC changes occurred for B prostate-cancer: 2y 50-74y rates first dropped, then showed a 1992-1999 nonsignificant rise and fall, followed by a significant 1999-2006 rise and a significant 20062010 fall; 5y rates showed a nonsignificant 1988-1991 fall and a significant 1991-2007 rise. For 2y 1988-2010 AAPC analyses, prostate-cancer rates lacked definite change for 50-74y men, and rose for 75+y men; ohter-cause rates lacked definite change for $75+\mathrm{y} \mathrm{W}$ and dropped for 50-74y W and 50+y B. For 5y 1988-2007 AAPC analyses, prostate-cancer rates showed no definite change for $50+\mathrm{y} \mathrm{B}$ or $50-74 \mathrm{y} \mathrm{W}$ and increased for $75+\mathrm{y} \mathrm{W}$; other-cause rates dropped for all subgroups.

Conclusions: Sustained prostate-specific mortality drops were not seen for men with metastatic prostate cancer. Posited prostate cancer mortality declines are explicable by other-cause mortality declines. The 2006-2010 prostate cancer rate fall for Black 50-74 y may reflect the introduction of Docetaxel.

1069 Comparison of GRP52, mTOR, HoxB13, NKX3.1 and Prostein in Metastatic Prostatic Adenocarcinoma

Hai Wang, Yiang Hui, Dongfang Yang, Kara A Lombardo, Evgeny Yakirevich, Murray Resnick, Lijuan Wang, Shaolei Lu. Brown University, Providence, RI.

Background: Metastatic prostate cancer is frequently presented as cancer of unknown origin. Immunohistochemical (IHC) stains of PSA and prostein are frequently used to confirm its origin. However, these two markers are under the regulation of androgen receptor (AR) and their expression could be suppressed by hormonal therapy or altered by chemoradiation. Additional sensitive and AR-independent prostate cancer markers will be diagnostically beneficial. After mining public gene expression databases, we identified 4 markers, GRP52, mTOR, HoxB13 and NKX3.1, and compared them to prostein in a series of metastatic prostate cancer.

Design: We collected 46 metastatic prostate cancers, including 16 bone metastases $(10$ treated by hormonal ablation and/or chemoradiation and 6 untreated) and 30 non-bone metastases ( 27 treated vs 3 untreated). IHC stains of 5 markers were performed on the whole tissue sections. Additional tissue microarrays of primary prostate carcinoma ( 70 cases) were included for comparison. Positivity was defined as $\geq 5 \%$ of tumor cells with unequivocal staining of appropriate pattern.

Results: In 27 treated non-bone metastasis cases, positive rates of GPR52, mTOR, NKX3.1, HoxB13 and Prostein were $100 \%, 92.6 \%, 87.5 \%, 83 \%$, and $67 \%$, respectively. Out of 9 cases that were negative for prostein, GPR52, mTOR, NKX3.1, and HoxB13 could be detected in $4,8,7$, and 6 cases, respectively. Out of 3 cases of untreated nonbone metastasis, GRP52 was negative in 1 and prostein was negative in 2, while mTOR, NKX3.1, and HoxB13 were positive in all of them. All 5 markers were detected in all 16 bone metastases, except that HoxB13 could not be detected in 2 of 10 cases of treated bone metastasis. GPR52, mTOR, NKX3.1, and prostein were immunoreactive in all cases of primary prostate cancer, while HoxB16 in in $87.9 \%$ of cases.

Conclusions: Hormonal and/or chemoradiation have significant impacts on the expression of prostate markers. Expression of a commonly used marker, prostein, is significantly altered in treated prostate metastasis. Combination of multiple markers may increase the sensitivity. Decalcification has minimal impact on the detection of major prostatic cancer markers.

1070 Neoadjuvant Chemotherapy (NAC)-Related Histological Changes in Radical Cystectomy (RC): Assessment Accuracy and Prediction of Response

Hui Jun Wang, Wassim Kassouf, Fadi Brimo. McGill University Health Center, Montreal, QC, Canada.

Background: NAC is increasingly used in urothelial carcinoma. Traditionally, the assessment of NAC effect is based on evaluating the residual viable tumor in relation to the macroscopic tumor size. However, evaluating NAC-effect in RCs is confounded by the transurethral resection (TUR)-related histological changes. We evaluated whether NAC results in unique histological features to determine whether cases with no/minimal residual disease on RC can be attributed to NAC or TUR effect or both.

Design: 25 patients who received NAC were divided based on both their pre-operative clinical/radiographic findings (clinical stage; hydronephrosis; palpable mass) and the $\mathrm{RC}$ findings into NAC-respondents (advanced clinical stage and $<\mathrm{pT} 2+\mathrm{pN} 0$ ), possible NAC-respondents (non-advanced clinical stage and $<\mathrm{pT} 2+\mathrm{pN} 0$ ) and NACnon-respondents $(>\mathrm{pT} 2 \mathrm{and} / \mathrm{or}>\mathrm{pN} 1)$. In addition, 14 patients who received TUR alone and had $<\mathrm{pT} 2+\mathrm{pN} 0$ on RC were included as TUR alone arm. Presence/absence of the following histological features was assessed in all RCs: fibrosis/myofibroblastic reaction, hyalinisation in the bladder wall, prominent inflammatory reaction, calcification, foreignbody giant cells, necrosis, sheets of foamy macrophages, and fibrosis/hyalinisation/ necrosis in the lymph nodes (LN).

Results: Overall, there was a significant histological overlap between all groups. However, patients who received NAC had significantly higher likelihood of showing hyalinisation $(9 / 25$ versus $0 / 14 ; \mathrm{p}=0.01)$ and less giant cells $(\mathrm{p}=0.002)$ and inflammatory $(\mathrm{p}=0.01)$ reaction in comparison to those who received TUR only. In comparison to non-respondents to NAC, NAC-respondents showed significant hyalinisation $(\mathrm{p}=0.03)$ and fibrosis/necrosis in the LNs $(\mathrm{p}=0.006)$. Moreover, the only significantly different histological features in NAC-respondents versus TUR-respondents were hyalinisation $(\mathfrak{p}=0.0004)$ and $\mathrm{LN}$ changes $(\mathrm{p}=0.003)$, with those two features being present in two (25\%) and no case of the possible NAC-respondents group, respectively. Lastly, there was no significant difference in the possible NAC-respondents group in comparison to the TUR-only arm.

Conclusions: TUR and NAC result in histological changes with significant overlap. In cases with no/minimal residual disease on $\mathrm{RC}$, it is difficult to attribute the changes to NAC-effect-only except if: 1- hyalinisation of the bladder wall or LN changes are present, or 2-if the pre-operative clinical stage was beyond what could be resected by TUR (example: >cT2; >cN0). Those findings should be taken in consideration in studies evaluating response to NAC in UC.

1071 Comparison of Margin Status and Clinical Follow-Up between Simple Tumor Enucleation and Partial Nephrectomy of Small Renal Tumors Lu Wang, Ian Hughes, Connor Snarskis, Gopal Gupta, Maria M Picken. Loyola University Chicago, Maywood, IL; Loyola University, Maywood, IL; Stritch School of Medicine, Maywood, IL.

Background: Both partial nephrectomy (PN) and tumor enucleation (TE) are acceptable modalities for removal of small renal tumors, while preserving nephrons. However, the optimal surgical margin is contested because of concern about the oncologic adequacy of surgical margins. Our objective was to compare the margin status, procedure parameters, postoperative complication and tumor recurrence.

Design: All consecutive renal TE and PN specimens obtained during the period 06/2012 to $06 / 2015$, with an available clinical follow-up, were included in this study. Particular attention was paid to features of pseudocapsule, margin status, procedure parameters, complication and imaging follow-up for tumor recurrence. Data was analyzed using the student's t-test and the two-tailed Fisher's exact test.

Results: A total of 114 tumor specimens (58 TE, $56 \mathrm{PN}$ ) were reviewed, including 69 clear cell type renal cell carcinomas (RCC), 19 papillary RCC, 10 oncocytomas, 8 angiomyolipomas, 6 chromophobe RCC, 1 mucinous tubular and spindle cell RCC and 1 paraganglioma. The average tumor size was $3.0 \mathrm{~cm}$ (range 1.0-6.5 cm). Ten of 58 renal tumors removed with TE $(17.2 \%)$ showed surgical margin positivity, compared to none of 56 renal tumors removed with PN. Compared to PN, TE involved a significantly shorter procedure time, lesser blood loss and fewer post-operative complications. During imaging follow-up, none of the patients from either group was found to have a local recurrence. 


\begin{tabular}{|l|l|l|l|}
\hline & TE & PN & p-value \\
\hline average margin distance & $0.27 \mathrm{~mm}$ & $2.84 \mathrm{~mm}$ & $<0.001$ \\
\hline specimen with positive margin & $10(17.2 \%)$ & $0(0 \%)$ & 0.001 \\
\hline number of procedures without hilar clamp (\%) & $31(52.5 \%)$ & $0(0 \%)$ & $<0.001$ \\
\hline procedure time & $181 \mathrm{mins}$ & $241 \mathrm{mins}$ & $<0.001$ \\
\hline blood loss during procedure & $180 \mathrm{ml}$ & $280 \mathrm{ml}$ & $<0.001$ \\
\hline average hospital stay & 1.71 days & 2.67 days & $<0.001$ \\
\hline $\begin{array}{l}\text { patient need re-admission of hospital within 1 } \\
\text { month (\%) }\end{array}$ & $0(0 \%)$ & $7(12.1 \%)$ & 0.006 \\
\hline median follow up months (range) & $22(11-40)$ & $19(8-42)$ & 0.842 \\
\hline tumor recurrence & 0 & 0 & 1 \\
\hline
\end{tabular}

Conclusions: TE maximally preserves the uninvolved kidney parenchyma and causes less post-operative complications. Although positive surgical margins were more frequently encountered in TE specimens, local tumor recurrence was comparable to PN. However, a longer period of follow up is warranted for complete evaluation of tumor recurrence.

1072 Characterization of Papillary Hyperplasia in Nephrectomy Specimens from Patients with Autosomal Dominant Polycystic Kidney Disease - A Study of 60 Cases

Lu Wang, Rajen Goyal, Ondrej Hes, Ximing Yang. Northwestern University, Feinberg School of Medicine, Chicago, IL; Charles University Hospital, Alej Svobody, Plzen, Czech Republic.

Background: Papillary hyperplasia $(\mathrm{PH})$ is frequently seen in nephrectomy specimens in autosomal dominant polycystic kidney disease (ADPKD) patients. The frequency and its potential to develop into renal cell carcinoma (RCC) from this lesion is uncertain. Only sparse data have been published regarding to the incidence of RCC developed in ADPKD. This study aims to evaluate the frequency and pathologic feature of papillary hyperplasia as well as the incidence of RCC developed from ADPKD.

Design: Sixty nephrectomy specimens with ADPKD from 01/2010 to 09/2015 were included in this study. Special attentions were paid to the presence of $\mathrm{PH}$ on the cystic wall, cortical papillary adenoma and any RCC. The immunoprofile of PH was explored by immunohistochemical stains for CK7, AMACR and CA-IX. Staining pattern is graded by combined score of both intensity ( 0 : negative; 1 : weak; 2 : moderate; 3 : strong) and extent $(0:<1 \% ; 1: 1-10 \% ; 2: 10-50 \% ; 3:>50 \%)$ with the maximum score of 6 . Data was analyzed by Microsoft Excel.

Results: A total of 60 nephrectomy specimens from 48 patients of average age 49.2 year old, were evaluated. Fourteen patients had history of dialysis with period of 0.25 to 11 months. PH,was found in all 60 specimens $(100 \%)$, while cortical papillary adenoma and RCC were found in $9 / 60(15 \%)$ and $1 / 60(1.67 \%)$ specimens. The single case of RCC was classified as papillary RCC from a patient with 4 month of dialysis. For each individual ADPKD nephrectomy case, $\mathrm{PH}$ was found in an average of $58.2 \%$ of submitted blocks (range from $33.3 \%$ to $100 \%$ ). All PH showed diffuse and strong cytoplasmic positivity for CK7 (score 5.87), while largely negative for AMACR (score 0.32). CA-IX shows weak cytoplasmic stain (score 2.58) in most of cases, except one with cup-shaped membrane stain.

Conclusions: Our study showed a high prevalence of papillary hyperplasia (100\%) and low prevalence of RCC (1.67\%) in nephrectomy specimens from patients with ADPKD. The immunostainging pattern of $\mathrm{PH}$ is unique and different from that of papillary adenoma, papillary RCC or clear cell RCC. Our finding suggests a low potential of papillary hyperplasia to develop into RCC. Papillary hyperplasia should not be confused with other papillary neoplastic lesions in a kidney with ADPKD.

1073 Invasion of Rete Testis, Hilar Fat, and Epididymis by Testicular Malignant Germ Cell Tumors (MGCT) Does Not Justify Upstaging to pT2 Elizabeth Whittington, Nicholas Harding Jackson, Nicola Pavan, Samarpit Rai, Kenneth A Iczkowski, Oleksandr N Kryvenko, Merce Jorda. University of Miami/ Jackson Memorial Hospital, Miami, FL; Medical College of Wisconsin, Milwaukee, WI. Background: The significance of rete testis, hilar fat, or epididymis involvement by MGCT is controversial. AJCC cancer staging $\left(7^{\text {th }}\right.$ edition) classifies involvement of any of these structures as pT1, however, disagreement persists as to whether these findings, in the absence of vascular invasion, warrants a pT2 stage (PMID:25619976). Some studies suggest that rete testis involvement is associated with higher rate of metastasis (PMID: 23238629). A cohort of testicular MGCT were studied to clarify the significance of involvement of these structures in pathologic staging.

Design: We reviewed consecutive $\mathrm{pT} 1$ and $\mathrm{pT} 2$ orchiectomies with MGCTs performed between 2000-13 in 2 academic institutions. Standard grossing techniques were used. pT1 neoplasms were subdivided into those with involvement of rete testis, hilar fat, and/or epididymis (pT1-inv), and those without involvement (pT1 NO-inv). Correlation with recurrence/metastasis (RM) was assessed using Fisher's, Anderson-Darling, and Kruskal-Wallis tests for statistical analysis.

Results: Results are displayed in the table. Patients with pT1-inv do not demonstrate significant higher rate of RM than $\mathrm{pT} 1 \mathrm{NO}$-inv $(\mathrm{p}=0.165)$. A trend to higher rate of RM in pT2 neoplasms compared to pT1-inv was identified ( $\mathrm{p}=0.115)$.

\begin{tabular}{|c|c|c|c|c|c|c|}
\hline \multirow{2}{*}{\multicolumn{2}{|c|}{ Parameter }} & \multirow{2}{*}{$\begin{array}{c}\text { General } \\
(\mathrm{n}=157)\end{array}$} & \multicolumn{4}{|l|}{ Study group } \\
\hline & & & $\begin{array}{l}\text { pT1 NO-inv } \\
(\mathrm{n}=85)\end{array}$ & $\begin{array}{l}\text { pT1-inv } \\
(n=21)\end{array}$ & $\begin{array}{l}\mathrm{pT2} 2 \\
(\mathrm{n}=51)\end{array}$ & $\mathrm{p}$-value \\
\hline \multicolumn{2}{|c|}{ Age, years } & $\begin{array}{l}31(32.9, \\
14-83)\end{array}$ & $\begin{array}{l}30.5(32.3 \\
16-62)\end{array}$ & $\begin{array}{l}34(35.3, \\
19-81)\end{array}$ & $\begin{array}{l}31(32.9, \\
14-83)\end{array}$ & 0.78 \\
\hline \multicolumn{2}{|c|}{ Follow-up, months } & $\begin{array}{l}50.7(56.1, \\
2-180.4)\end{array}$ & $\begin{array}{c}51.8(59.5 \\
2.8-180.4)\end{array}$ & $\begin{array}{c}55.5(61.4 \\
39-100.4)\end{array}$ & $\begin{array}{l}35(48.3, \\
2-160)\end{array}$ & 0.087 \\
\hline \multicolumn{2}{|c|}{$\begin{array}{l}\text { Recurrence/ } \\
\text { Metastasis }\end{array}$} & $55(35 \%)$ & $27(32 \%)$ & $5(24 \%)$ & $23(45 \%)$ & 0.165 \\
\hline \multirow{4}{*}{$\begin{array}{l}\text { Tumor } \\
\text { type }\end{array}$} & Seminoma & $81(52 \%)$ & $43(51 \%)$ & $14(67 \%)$ & $24(47 \%)$ & \multirow{4}{*}{0.235} \\
\hline & Embryonal & $13(8 \%)$ & $4(5 \%)$ & $1(5 \%)$ & $8(16 \%)$ & \\
\hline & Mixed & $61(39 \%)$ & $36(42 \%)$ & $6(28 \%)$ & $19(37 \%)$ & \\
\hline & Teratoma & $2(1 \%)$ & $2(2 \%)$ & - & - & \\
\hline
\end{tabular}

Data in median (mean, range)

Conclusions: pT1 testicular MGCT with or without involvement of rete testis, hilar fat, and/or epididymis do not have a significant difference in rate of RM. Even though a trend is noted to have higher rate of RM in pT2 tumors in comparison to pT1 neoplasms with involvement of the structures, this was not significant. These data support current AJCC cancer staging guidelines, where involvement of rete testis, hilar fat, and/or epididymis by MGCT without vascular invasion is staged as $\mathrm{pT} 1$ neoplasm.

\section{Diagnostic Criteria for Oncocytic Renal Neoplasms: A Survey of} Specialist Renal Tumor Pathologists

Sean R Williamson, Ramya Gadde, Kiril Trpkov, Michelle Hirsch, John R Srigley, Victor E Reuter, Liang Cheng, L Priya Kunju, Ravi Barod, Craig G Rogers, Brett Delahunt, Ondrej Hes, John N Eble, Ming Zhou, Jesse K McKenney, Guido Martignoni, Stewart Fleming, David J Grignon, Holger Moch, Nilesh S Gupta. Henry Ford Health System, Detroit, MI; McMaster University, Toronto, Canada; Memorial Sloan Kettering Cancer Center, New York, NY; Indiana University, Indianapolis, IN; University of Michigan, Ann Arbor, MI; Wellington School of Medicine, Wellington, New Zealand; Charles University, Plzen, Czech Republic; New York University, New York, NY; Cleveland Clinic, Cleveland, OH; University of Verona, Verona, Italy; University of Calgary, Calgary, Canada; Brigham and Womens Hospital, Boston, MA; University of Dundee, Dundee, United Kingdom; University Hospital Zurich, Zurich, Switzerland. Background: Renal oncocytoma and chromophobe renal cell carcinoma (RCC) have been long recognized as distinct tumor subtypes; however, it remains unclear if uniform histologic, immunohistochemical, and genetic diagnostic criteria are used in practice. Design: Urologic pathologists with subspeciality interest in the kidney were invited to participate in a survey of diagnostic criteria for oncocytic renal tumors. Results: Responses were received from 17/26 invitees. Histologically, $>1$ mitotic figure was regarded as most uniformly worrisome $(n=10)$ or incompatible with oncocytoma diagnosis $(n=6)$, whereas focal nuclear wrinkling, focal perinuclear clearing, and multinucleation did not necessarily exclude this diagnosis but may, dependent on extent. Staining techniques most commonly used included: CK7 (94\%), KIT (71\%), vimentin (65\%), colloidal iron (59\%), CD10 (53\%), and AMACR (41\%). Rare CK7-positive cells $(\leq 5 \%)$ was regarded as most supportive of oncocytoma, although a staining threshold excluding this diagnosis was not universal. Multiple chromosomal loss was most strongly supportive for chromophobe RCC diagnosis (65\%); however, less certainty was reported for gains or a single loss. For tumors with mixed or inconclusive features, many participants use an intermediate diagnostic category $(82 \%)$ that does not label the tumor as unequivocally benign or malignant, typically "oncocytic neoplasm" or "tumor" with a description. Some (65\%), but not all, report outright diagnosis of oncocytoma in needle biopsies. A small minority (3/17) reported having seen oncocytoma-like tumors that metastasized.

Conclusions: The morphologic, immunohistochemical, and genetic characteristics that define oncocytic renal tumors remain incompletely understood. Further studies correlating genetics, outcome, and histology are needed to define which tumors truly warrant classification as carcinomas for patient counseling and follow-up strategies.

1075 Chromosome 6p Amplification Including the TFEB Gene: A Novel Mechanism of Renal Cell Carcinoma Pathogenesis?

Sean R Williamson, David J Grignon, Laura Favazza, Nilesh S Gupta, Dhananjay A Chitale, Nallasivam Palanisamy. Henry Ford Health System, Detroit, MI; Indiana University, Indianapolis, IN.

Background: Amplification of part of chromosome 6p, which includes almost half of the genes from chromosome 6 , has been implicated in aggressive behavior in multiple cancers, although not to our knowledge in renal cell carcinoma. In this study, we report on a cohort of 3 renal cell carcinomas with $6 \mathrm{p}$ amplification including the TFEB gene, a member of the microphthalmia transcription factor (MITF) family of genes.

Design: Three renal cell carcinomas were identified as having amplification of chromosome $6 \mathrm{p}$ including the TFEB gene, 1 via fluorescence in situ hybridization (FISH), and 2 from the Cancer Genome Atlas (TCGA) database. We studied morphology, immunohistochemical staining characteristics, and other genetic alterations in this cohort.

Results: The patients were 2 women and 1 man, ages 28,61 , and 57, respectively. All 3 tumors were infiltrative (pT3a) tubulopapillary neoplasms with variable infiltration of renal sinus fat, renal parenchyma, or vessels (A-B). The youngest patient had regional nodal metastasis at presentation. Amplification of the TFEB region was observed by FISH (C) in 1 tumor, without TFEB or TFE3 rearrangement or loss of chromosome $3 p$ (D). The two TCGA specimens showed selective amplification of a region of chromosome 6p including the TFEB gene, without corresponding amplification of the MALAT1 (Alpha) gene region, the known fusion partner in TFEB translocation. VHL 
mutation and chromosome $3 \mathrm{p}$ loss were not observed, although the young patient had somatic mutation in $F H$ (germline status unknown). $F H$ mutation was lacking in the other TCGA tumor. The 2 tumors with immunohistochemical results were positive for CD10, AMACR, and PAX-2 or PAX-8. One demonstrated patchy positivity for melan-A and cathepsin K, whereas the other was negative for HMB45 and melan-A.
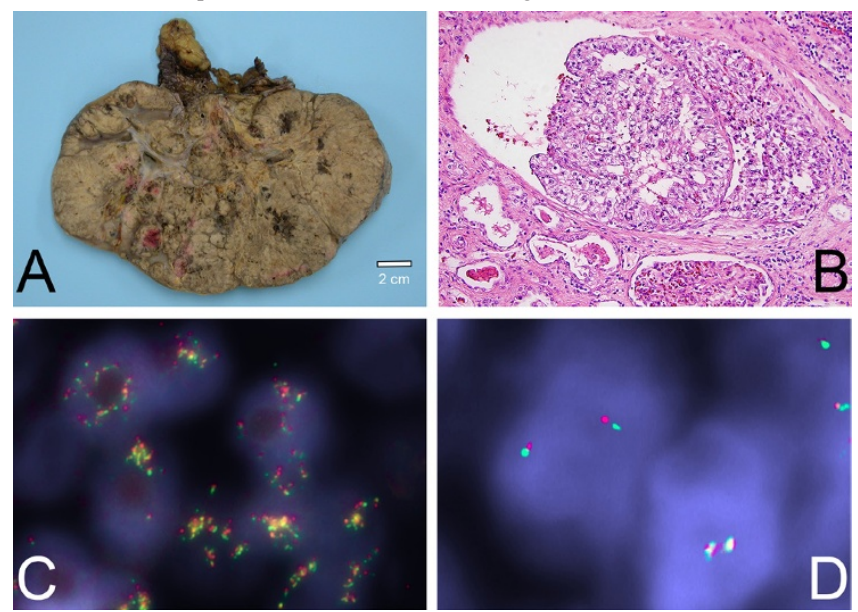

Conclusions: In this cohort, $6 \mathrm{p}$ amplification was associated with aggressive tubulopapillary histology and lack of genetic alterations of other tumor types. One patient had $\mathrm{FH}$ gene mutation in the tumor, although it remains to be studied whether this is exclusively somatic or a manifestation of the hereditary leiomyomatosis syndrome. Further studies will define whether this represents a unique entity related to MITF gene family alteration.

\section{Clear Cell Renal Cell Tumors with Intact VHL and Chromosome 3p:} How Many Entities Exist?

Sean R Williamson, Dhananjay A Chitale, Laura Favazza, Ravi Barod, Craig G Rogers, Nallasivam Palanisamy, Nilesh S Gupta. Henry Ford Health System, Detroit, MI. Background: Clear cell renal cell carcinoma (RCC) is the most common adult renal cancer. However, there is increasing recognition that other tumors with morphologic similarity are genetically and likely behaviorally distinct, as exemplified by clear cell papillary RCC. We hypothesize that there may be yet other renal tumors currently classified as clear cell RCC that have distinct genetic underpinnings and possibly unique histologic features.

Design: The Cancer Genome Atlas (TCGA) published dataset was queried (cbioportal. org) for clear cell RCC tumors lacking $V H L$ gene mutation and chromosome $3 p$ loss. Genetic alterations were correlated with whole-slide images.

Results: Of 418 tumors in the published TCGA clear cell RCC database, 387 had $V H L$ mutation, copy number loss for chromosome $3 p$, or both $(93 \%)$. Of the remaining 31 tumors, 27 had whole-slide images for review. Of these, 3 were shown in another study to be translocation RCC, all of which had suggestive histology, including large nests with calcifications, eosinophilic cells, papillae, and sclerotic stroma. Notably, one SFPQ-TFE3 tumor identified by the same study also had $V H L$ mutation, copy number loss for chromosome $3 \mathrm{p}$, and morphology indistinguishable from clear cell RCC. Two tumors had highly vacuolated cell morphology, of which 1 had copy number gain for chromosome 17, suggesting these may represent papillary RCC with florid cytoplasmic vacuolization. One had morphology of clear cell papillary RCC, and 3 had TCEB1 mutation, the features of which have recently been published. Four were high-grade RCC with rhabdoid morphology, and 5 had prominent fibromuscular stroma. The remaining 9 tumors lacked a unifying histologic pattern; however, these exhibited minor unusual findings, including foamy rather than empty-appearing cytoplasm $(n=4)$, widespread nuclear alignment $(n=1)$, intranuclear cytoplasmic invaginations $(n=1)$, and hyalinized stroma $(\mathrm{n}=1)$. Of all 27 tumors, only 6 had other mutations recently recognized in clear cell RCC, including SMARCA4 $(\mathrm{n}=2), \operatorname{SETD} 2(\mathrm{n}=2), P B R M 1(\mathrm{n}=1)$, and BAP1 $(\mathrm{n}=1)$. Conclusions: The vast majority of clear cell RCC harbor $V H L$ mutation, 3 p copy number loss, or both. Of tumors with clear cell histology that lack these alterations, a subset can likely be reclassified as other established or emerging tumor entities. The remaining tumors, such as those with de novo high-grade morphology, fibromuscular stroma, and other subtle histologic variations suggest that a "clear cell" appearance may be a common phenotype of several genetic pathways, for which improved understanding may define new entities and guide prognostication and treatment.

1077 Clinical Follow-Up of Renal Oncocytomas with Vascular Invasion Sara E Wobker, Jonathan I Epstein. The Johns Hopkins Medical Institutions, Baltimore, MD.

Background: Renal oncocytomas are benign renal neoplasms that are often excised as clinically they cannot be distinguished with certainty from renal cell carcinoma. One of the least common findings in oncocytomas is vascular invasion. The behavior of oncocytomas with vascular invasion is not well-characterized with only reports of isolated examples.

Design: We searched for cases of renal oncocytoma with vascular invasion that were seen in consultation at our institution since 2000

Results: Of 699 cases of renal oncocytoma diagnosed on the consult service, $14(2 \%)$ had vascular invasion. Slides were available for review in 12 cases. Patients included
8 men and 6 women with an average age of diagnosis of 67.8 years (range 48-91 years). Diagnostic specimens included 1 biopsy, 2 partial nephrectomies and 11 radical nephrectomies. Tumor size ranged from $1.0-13.5 \mathrm{~cm}$ (mean $6.0 \mathrm{~cm}$ ). Five cases had co-existing neoplasms in the diagnostic specimen: 3 clear cell renal cell carcinomas and 2 angiomyolipomas. All specimens, with the exception of one tubulocystic type, showed at least some component of classic nested architecture. Focal or pronounced degenerative atypia was present in 6 cases (Figure 1).

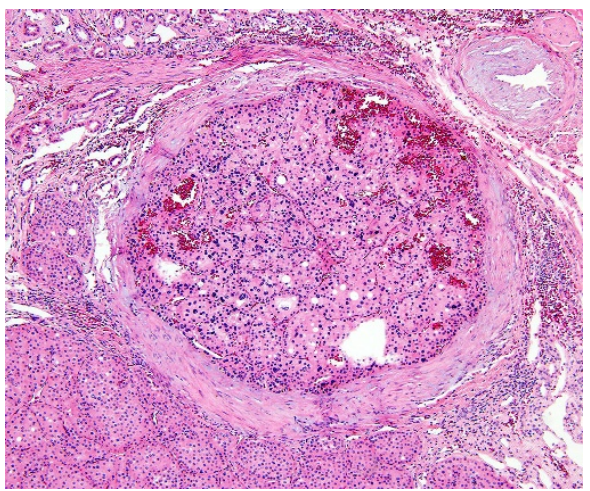

Invasion of perirenal fat was identified in 4 cases. Seven cases showed large vessel invasion and the remainder involved medium to small vessels. Tumor was grossly visible in the renal vein in 2 cases. Clinical data was available on 10 of the 14 cases with an average follow-up time of 44.3 months (range 3.9-94.8 months). Of the cases with clinical follow-up, all but one individual was alive. Records were not available as to the cause of death for this patient. All living individuals were free of recurrence or metastatic disease at the time of last follow-up.

Conclusions: This study confirms that oncocytomas are benign entities, even in cases with vascular invasion. Our cohort showed no metastasis or recurrence and overall survival of $90 \%$ at 3 years post-diagnosis, supporting the finding that vascular invasion does not appear to alter the favorable prognosis. The presence of vascular invasion by classic oncocytoma should not lead to any uncertainty about the diagnosis.

1078 Diversity of Resistance Mechanisms in Prostate Cancer after Neoadjuvant Intense Androgen Deprivation Therapy

Huihui Ye, Adam G Sowalsky, Rachel Schaefer, Xin Yuan, Shao-yong Chen, Sean J Gerrin, Laleh Montaser, Fen Ma, Olga Voznesensky, Changmeng Cai, Rosina Lis, Zhenwei Zhang, Massimo Loda, Mary-Ellen Taplin, Steven Balk. Beth Israel Deaconess Medical Center, Boston, MA; Dana-Farber Cancer Institute, Boston, MA.

Background: In a randomized phase II neoadjuvant clinical trial, 58 patients with localized, intermediate to high risk prostatic adenocarcinoma received intense androgen deprivation therapy (IADT) combining leuprolide with abiraterone acetate prior to surgery. In this study, we assessed early IADT resistance mechanisms in residual tumors in the radical prostatectomy specimens, with a particular interest in intratumoral heterogeneity.

Design: Residual tumor cells and paired benign glands were laser microdissected from 19 trial cases, with two distant tumor foci microdissected from 14 cases. We examined tumor genetic alterations using whole exome sequencing and gene expression profiles using Affymetrix microarray. Intratumoral heterogeneity and clonality were further examined using immunohistochemical and in situ RNA detection assay in 50 trial cases and 40 untreated control cases for total AR, AR-V7, pS81AR, NKX3.1, ERG, Rb, p53, PTEN, pAkt, pS6, p27, pHer3, SOX9, synaptophysin, chromogranin, and Ki67. Results: The treated tumors showed remarkable diversity of resistance mechanisms, including foci-specific genetic alterations such as tumor suppressor losses, activation mutations in oncogenes, and foci-specific dysregulation of gene expression (including overexpression of AR-V7) and pathway activation. Diversity was also reflected in drastic variation in neuroendocrine differentiation and proliferation indexes among tumor foci. A few common resistant mechanisms were identified, including homogeneous upregulation of SOX9, homogeneous nuclear expression of $\mathrm{pS} 81 \mathrm{AR}$, and persistent expression of AR signature.

Conclusions: Our study suggests that pre-existing tumor subclones may survive and expand under selection pressure in the IADT setting and serve as early resistance mechanisms. Our study also indicates that persistent AR activation and SOX9 overexpression are potential therapeutic targets to overcome early IADT resistance.

1080 The Correlation of TP53 and RB1 Genetic Alterations and Immunohistochemical (IHC) Expression in Invasive and Non-Invasive Urothelial Carcinoma

Xiaoyong Zheng, Qinghu Ren, Judy Sarungbam, Emmet J Jordan, Satish K Tickoo, Samson Fine, Ying-Bei Chen, Michael F Berger, Anuradha Gopalan, S Joseph Sirintrapun, Gopa Iyer, Byron H Lee, Eugene K Cha, Michael F Berger, David B Solit, Victor E Reuter, Hikmat Al-Ahmadie. Memorial Sloan Kettering Cancer Center, New York, NY.

Background: Urothelial carcinoma (UC) is a genetically heterogeneous disease. Lossof-function mutations of TP53 and RB1 are common in UC. While TP53 mutations are known to occur early, most literature points to $R B 1$ mutations as late events developing in the invasive disease. In a cohort of UC that has been genetically profiled, we investigated the occurrence of TP53 and RBI mutations and IHC correlates in both the invasive and non-invasive disease. 
Design: Tissue microarrays (TMAs) were constructed from genetically profiled tumors (3 TMAs, $\mathrm{n}=149$ ), of which 34 have TP53 mutations and 20 have RB1 mutations or deletion. We performed immunohistochemistry for $\mathrm{p} 53$ and RB on all TMA slides as well as on full sections form another set of tumors with TP53 $(\mathrm{n}=10)$ and $R B(\mathrm{n}=14)$ mutations. These full sections contained both invasive and non-invasive UC to compare the expression patterns between these 2 components.

Results: From the TMAs, in 34 tumors with known TP53 mutation, p53 expression was strong and diffuse in 19 (19/34), was completely negative in 10 cases $(10 / 34)$, and focally/weakly positive in 5. In 20 tumors with known $R B 1$ mutation, $\mathrm{RB}$ expression was completely lost in 17 cases $(17 / 20)$, and focally/weakly positive in 3 cases. Cases that were TP53/RB1 wild type exhibited a spectrum of expression but generally none were diffusely and strongly positive for p53 or completely negative for RB. RB expression was lost in both the invasive and non-invasive components in all RB1 mutated tumors (adjacent normal urothelium and stromal cells retained RB expression). Similarly, in TP53 mutated tumors, the patterns of expression were similar between the invasive and non-invasive components.

Conclusions: Although TP53 mutations are well-known to be early events in UC, we demonstrate that $R B 1$ loss is an early event in the development of urothelial carcinoma. $R B 1$ mutations result in complete loss of $\mathrm{RB}$ expression in overwhelming majority of the cases. TP53 mutations may result in either diffuse and strong p53 expression or, less frequently, complete loss of expression. Awareness of these patterns of expression may be helpful in assessing the potential role of these markers in patient risk stratification.

1081 Mutational Analysis of Gleason Score 7 Prostate Cancer Using Next Generation Sequencing and Fluorescent In Situ Hybridization

Ming Zhou, Zach Liu, Roberto Zoino, Chrysostomos Chrysostomou, Gaurav Rajoria, Szczepan Klimek, Herber Lepor. New York University, New York, NY; Histopathology Services, Ramsey, NJ.

Background: Prostate cancer (PCa) is a clinically and genetically heterogeneous disease. Understanding the genetic abnormalities is necessary to better determine the risk of disease progression and to identify potential targets for precision therapy. We analyzed the mutational landscape in a cohort of intermediate grade (Gleason score 7) PCa with well characterized clinical fellow-up.

Design: 42 cases of GS7 PCa were studied. H\&E sections were reviewed to confirm the diagnosis. Representative tumor areas were microdisected. DNA was extracted from the paraffin sections. DNA library was prepared with PCR amplification. Each patient sample was barcoded and loaded for emulsion PCR for clonal amplification. The amplified DNA copies were then sequenced on the Ion torrent PGM. The sequences were analyzed with variant caller software. 19 genes that have been repeatedly shown to be mutated in PCa ("prostate genes" including APC, AR, FAT3, FOXA1, KLK2 „KLK3, KLK4, MED12, MUC16, MUC2, MUC4, SCN11A, SPOP, THSD7B, TNXB, TP53, TTN, ZAN and ZNF595) and Ion Torrent HotSpot Cancer panel that contained genes frequently altered in other cancers were sequenced. FISH on paraffin-embedded tumor tissue was performed using commercially available DNA probes for PTEN, ERG, and PIK3CA.

Results: Using FISH to detect ERG/TMPRSS2 translocation and PTEN deletion, 12 (30\%), $29(71 \%), 7(18 \%)$ and 7 (18\%) tumors had alteration involving ERG, PTEN, both genes and none of the genes. One of 37 tumors had PIK3CA amplification while none had AR amplification. None of these changes correlated with PSA recurrence. Using NGS to sequence the "prostate genes", 16 mutations were detected in 15 of 42 tumors, averaging 0.4 mutation per tumor. These mutations involved SPOP gene in 6 tumors, TP53 in 4, MED1 in 2, FoxA1 in 2, AR in 1 and ZAN in 1. One tumor had two mutations, 14 had 1 mutation and remaining 27 had no mutations. Mutations in SPOP correlated marginally with PSA recurrence with PSA recurrence in 0 of 6 tumors with SPOP mutation and in 12 of $31(39 \%)$ tumors with wild type SPOP $(\mathrm{p}=0.064)$. Mutation in KIT was detected in $8 / 43(19 \%)$ tumors. KIT mutation occurred only in PTEN deleted tumors $(7 / 22,32 \%)$, but not in PTEN wild type tumor $(0 / 12, \mathrm{p}=0.062)$. Conclusions: Gene mutations are rare in GS 7 PCa. SPOP is mutated in 6/42 (15\%) of tumors and marginally correlates with PSA recurrence. Chromosomal alterations involving PTEN and ERG are much more frequent. Relationship between PTEN deletion and KIT mutation warrants further investigation.

1082 PD-L1 Protein Expression and PD-L1 Gene Amplification in Urothelial Carcinoma of the Urinary Bladder

Chunlai Zuo, Bhaskar VS Kallakury, Bryan Firestone, Olga Voronel, Christine E Sheehan, Tipu Nazeer, Jeffrey S Ross. Albany Medical College, Albany, NY; Georgetown University Hospital, Washington, DC.

Background: Urothelial carcinoma (UCB) is the most common primary neoplasm of the urinary bladder. PD-L1 (programmed death-ligand 1), a 40KDa type 1 transmembrane protein, expression has been linked to enhanced response to the checkpoint inhibitor atezolizumab in UCB, but has not been evaluated as a prognostic factor for the disease. Design: Formalin-fixed paraffin-embedded tissue sections from 63 UCB were immunostained by an automated method utilizing rabbit monoclonal PD-L1/CD274 (Spring Bioscience, clone SP142) and ultraView DAB detection (Roche/Ventana, Tucson AZ). Membranous PD-L1 immunoreactivity was scored as negative, low positive (1 $-24 \%$ ) and high positive ( $=$ or $>25 \%$ ) in both the tumor cells and tumor infiltrating lymphocytes (TILs). Results were correlated with clinicopathologic variables. $P D-L 1$ gene amplification was assessed by hybrid capture-based comprehensive genomic profiling (CGP) on a separate set of $806 \mathrm{UCB}$.

Results: PD-L1 immunoreactivity was noted as positive in 8/63 (13\%) UCB [6 low positive and 2 high positive] and correlated with high tumor grade $[8 / 21(38 \%)$ grade 3 vs $0 / 21$ grade 2 vs $0 / 21(0 \%)$ grade $1, \mathrm{p}<0.0001]$ and advanced tumor stage $[6 / 23(26 \%)$ advanced (pT2 and pT3) versus $2 / 40(5 \%)$ low (pTa and pT1) stage, $\mathrm{p}=0.016]$. PD-L1 immunoreactivity was noted in the TIL component of $4 / 63(6 \%)$ UCB and correlated with high tumor grade [4/21 (19\%) grade 3 vs $0 / 21(0 \%)$ grade 2 vs $0 / 21(0 \%)$ grade 1 , $\mathrm{p}=0.014]$, while showing a trend for advanced tumor stage [3/23 $(13 \%)$ advanced vs $1 / 40$ (3\%) low stage, $\mathrm{p}=0.098]$. On CGP, $6(0.7 \%)$ of 806 UCB demonstrated PD-L1 gene amplification. On multivariate analysis, advanced tumor stage $(\mathrm{p}=0.007)$ independently predicted overall survival.

Conclusions: High tumor expression of PD-L1 was associated with high grade and advanced stage in UCB. $P D-L 1$ amplification is extremely uncommon in UCB indicating that $\mathrm{PD}-\mathrm{L} 1$ expression can occur independently of $P D-L 1$ gene copy number. This study confirms that PD-L1 expression is an adverse prognostic factor in UCB and supports the further development of checkpoint inhibitor therapy for this disease.

\section{Gynecologic and Obstetric Pathology}

1083 Expression of Alcohol Dehydrogenase 5 in Ovarian Carcinoma: Effect on Prognosis and Therapeutic Potential

Eman Abdulfatah, Giri Shailendra, Rattan Ramandeep, Mohammed F Daaboul, Sharif Sakr, Baraa Alosh, Muhammad K Alsafadi, Fatimah Alruwaii, Vishakha Pardeshi, Sudeshna Bandyopadhyay, Adnan R Munkarah, Rouba Ali-Fehmi. WSU, Detroit, MI. Background: S-nitrosoglutathione (GSNO), a physiological nitrosylating agent significantly inhibits ovarian cancer (OCa) growth by promoting nitrosylation of various genes and inhibiting inflammation. GSNO is catabolized by alcohol dehydrogenase 5 (ALDH5) leading to reduction in the process of nitrosylation. Our aim was to evaluate ALDH5 expression in OCa and its relation with inflammatory markers expression and survival and to explore the role of ALDH5 inhibition in treating OCa.

Design: IHC of ALDH5 was performed on tumor tissue microarray for 360 OCa (292 serous, 12 endometrioid, 43 mucinous and 13 clear cell carcinoma). IHC for COX-2, iNOS, eNOS and NFkB was performed on a subset of cases $(n=120)$. Each marker was evaluated to establish H-score. Expression of ALDH5 in various OCa cell lines was determined using quantitative PCR. OCa cell lines were treated with ALDH5 inhibitor (N6022) in the presence or absence of GSNO and cell survival was assayed by MTT. We investigated the effect of GSNO in an immunocompetent isogenic mouse model of OCa which expressed lower level of ALDH5. Data was analyzed by Fisher exact test and Kaplan-Meier analysis.

Results: High ALDH5 expression was significantly associated with Type II OCa (HG serous and endometrioid carcinoma) vs. Type I (LG serous, LG endometrioid, mucinous and clear cell carcinoma) $(\mathrm{P}=0.003)$. High ALDH5 expression was significantly associated with increased $\mathrm{COX}-2(\mathrm{P}=0.001)$ and $\mathrm{NFkB}(\mathrm{P}=0.001)$ expression (table 1). The overall survival was shorter in patients with high ALDH5 expression (median; 32 vs. 49 months) without significant difference. Expression of ALDH5 in OCa cell lines showed inverse correlation with cytotoxic effect of GSNO. Inhibition of ALDH5 potentiated the cytotoxicity of sub-optimal doses of GSNO in OCa cell lines $(\mathrm{P}<0.05)$. In preclinical mouse model, oral administration of GSNO significantly attenuated tumor growth and ascites accumulation $(\mathrm{p}<0.01)$.

\begin{tabular}{|l|l|l|l|}
\hline & $\begin{array}{l}\text { Low ALDH5 } \\
\text { expression(n=88) }\end{array}$ & $\begin{array}{l}\text { High ALDH5 } \\
\text { expression(n=32) }\end{array}$ & P value \\
\hline Cox-2 expression & & & \\
\hline $\begin{array}{l}\text { Low } \\
\text { High }\end{array}$ & $\begin{array}{l}28(31.8 \%) \\
60(68.2 \%)\end{array}$ & $\begin{array}{l}0(0.0 \%) \\
32(100.0 \%)\end{array}$ & $\mathbf{0 . 0 0 1}$ \\
\hline NFkB expression & & & \\
\hline Low & $82(93.2 \%)$ & $0(0.0 \%)$ & $\mathbf{0 . 0 0 1}$ \\
High & $6(6.8 \%)$ & $32(100.0 \%)$ & \\
\hline iNOS expression & & & 0.264 \\
\hline Low & $30(34.1 \%)$ & $7(21.9 \%)$ & \\
\hline High & $58(65.9 \%)$ & $25(78.1 \%)$ & 0.443 \\
\hline eNOS expression & & $\begin{array}{l}31(96.9 \%) \\
1(3.1 \%)\end{array}$ \\
\hline $\begin{array}{l}\text { Low } \\
\text { High }\end{array}$ & $\begin{array}{l}79(90.0 \%) \\
9(10.0 \%)\end{array}$ &
\end{tabular}

Conclusions: High ALDH5 expression was significantly associated with Type II OCa and inflammatory mediators. Inhibition of ALDH5 could be a potential therapeutic target in $\mathrm{OCa}$

1084 Discoidin Domain Receptor 1 in Advanced Stage Ovarian Carcinoma: Prognostic Implications and Epithelial-to-Mesenchymal Transition Associations

Eman Abdulfatah, Marta Marco, Rafael Fridman, Vishakha Pardeshi, Sharif Sakr Robert T Morris, Sudeshna Bandyopadhyay, Rouba Ali-Fehmi. WSU, Detroit, MI. Background: Ovarian carcinoma (OCa) presents at advanced stages with metastatic disease within the peritoneal cavity; one of the theories being acquisition of an epithelial-to-mesencyhmal transition (EMT). Discoidin Domain Receptors (DDRs); DDR1 and DDR2, are receptor tyrosine kinases that play a role in tumorgenesis, primarily stimulated by collagens I-IV and are associated with increased invasiveness via promoting EMT. In this study, we evaluate DDR1 expression in advanced stage OCa to determine correlations with clinicopathologic features and potential associations with EMT.

Design: Retrospective review of 95 stages (III-IV) OCa was conducted. Clinicopathologic parameters were analyzed. IHC of DDR1, collagen type 1 alpha (COL1A1) \& EMT markers (vimentin, SNAIL, SLUG, ZEB1 \& loss of E- cadherin) was performed on TMA of 95 OCa; HG serous(75), LG serous(8), HG endometrioid(5), 